Global Environmental Change in Drylands: Ecohydrological Controls on Plant Communities

A Dissertation presented to the Graduate Faculty of the University of Virginia in Candidacy for the Degree of Doctor of Philosophy

Department of Environmental Sciences

\author{
University of Virginia
}

by

Kailiang Yu

Dec 2016 



\section{Abstract}

Drylands cover much of the terrestrial land surface and many people depend on them as sources of their livelihoods. In the past few decades human activities associated with fossil fuel burning, fertilizer applications, and land use change have dramatically increased atmospheric $\mathrm{CO}_{2}$ concentrations and other trace gases (i.e., $\mathrm{NO}$ and $\mathrm{NO}_{2}$ ) as well as atmospheric temperature, a trend that is expected to continue in the decades to come. Global climate models predict increased precipitation variability at intra-annual, interannual and decadal time scales, especially in dryland regions. These changes in environmental conditions combined with human activities have led and will lead to significant changes in vegetation cover and plant community composition, with important impacts on ecohydrological and geochemical processes, regional climate and the provision of ecosystem services such as livestock grazing, sheltering of the soil surface, and carbon sequestration. Two major shifts in the composition of dryland vegetation are associated with woody plant-grass interactions and the increase in the abundance of plants which conduct Crassulacean Acid Metabolism (CAM). CAM plants feature water storage, nocturnal $\mathrm{CO}_{2}$ uptake, photosynthetic plasticity, and a high water usage efficiency. Primary studies of CAM plants have aimed to engineer CAM modules/genetics into other functional groups (i.e., $\mathrm{C}_{3}$ ) with the purpose of improving plant water usage efficiency, plant productivity, bioenergy production, and carbon sequestration in a changing environment. However, the ecohydrological controls underlying these vegetation changes (particularly expansion of CAM plants) remain poorly understood. To this end, this dissertation examined the impacts of major global environmental change drivers on woody plant-grass interactions and the competitive relationships between CAM plants and other functional groups (i.e., $\mathrm{C}_{3}$ and $\mathrm{C}_{4}$ plants) in dryland regions. Through the analysis of the ecohydrological controls underlying these vegetation changes, I show that woody plant encroachment can substantially suppress grass production by the effect of lateral root spread and limitations in soil water and light. The work also demonstrated that grass invasions and interannual rainfall fluctuations could act in concert to induce the ecosystem transition from shurblands to the unvegetated state. Instead of these "winloss" scenarios associated with woody plant-grass interactions, my research also shows that hydraulic lift could be an important mechanism responsible for the coexistence of woody plants and grasses in savannas. In addition to using new mechanistic models integrated with field or 
satellite data, I conducted greenhouse experiments to show that: i) under $\mathrm{CO}_{2}$ enrichment and drought conditions Cylindropuntia imbricata (a constitute CAM plant) outcompeted Bouteloua eriopoda ( $\mathrm{C}_{4}$ grass), with which it coexists in semiarid ecosystems across the Southwestern United States; ii) drought and nitrogen deposition - which have been predicted to increase in the near future - could serve as important drivers of expansion of facultative CAM plants such as Mesembryanthemum crystallinum, which interact with Bromus mollis ( $\mathrm{C}_{3}$ grasses) in California's coastal grasslands; iii) competition with other functional groups may enhance (or suppress, in case of insufficient carbohydrate availability) CAM expression in M. crystallinum, thereby affecting its plasticity and ability to cope with biological stress. Collectively, my research clarifies the effects of major global change drivers and shed light on the ecophysiological and ecohydrological processes responsible for the expansion of CAM plants in drylands around the world, a phenomenon that, to date, has been largely ignored in the environmental science literature. Finally, I developed new mechanistic models to assess the potential links between the two major changes in dryland vegetation observed around the world, namely, woody plants encroachment and expansion of CAM plants. It is found that woody plants could directly and/or indirectly facilitate CAM plants in their access to soil water resources, while the high rate of hydraulic descent performed by woody plants in woody plant-CAM associations could turn the facilitation of CAM plants by woody plants into competition. The novel contribution of my research is to address the key knowledge gaps in dryland vegetation response to global drivers of environmental change. The experimental analyses and process-based models provide an integrated understanding of woody plant encroachment and CAM plant expansion, which are two major changes observed in dryland vegetation around the world. 


\section{Doctoral Dissertation Committee}

\section{Paolo D'Odorico (Advisor)}

Department of Environmental Sciences

\section{Michael Pace}

Department of Environmental Sciences

Epstein, Howard

Department of Environmental Sciences

\section{Zoran Grujic}

Department of Mathematics 
In dedication to my parents 


\section{ACKNOWLEDGEMENTS}

I would like to express the deepest appreciation to my adviser Paolo D'Odorico for his profound and continuous support in my Ph.D. study and research. This work would not have been possible without his guidance, encouragement, and motivation. I want to also thank my committee members Michael Pace, Epstein, Howard, Zoran Grujic for all of their helpful comments and suggestions. Empirical experiments developed in the greenhouses benefited from a number of collaborators, including Scott L. Collins, Amilcare Porporato, Jose D Fuentes, David Carr and Kate Tully. In these experiments I am also grateful for the help of data collection by Abinash Bhattachan, Alexander Hassler, Amber Bouchard, Ashden Personius, Lixin Wang, Jun Yin, Hannah Piester, Heather Landes, Heng Huang, Mark Bartlett, Peri Bowser, Philip Schwartz, Samantha Hartzell, Wei Li, William P. Gilhooly III, and Yongli He.

The greenhouse experiments were supported by the University of Virginia through the Vice President for Research Office (Graduate Environmental Resilience Fellowship), the Department of Environmental Sciences (Moore Research Grant), and Blandy Experimental Farm (Summer Fellowship). I would like to also acknowledge the support (fellowship) of China Scholarship Council.

My time at the university has been blessed with a friendly and cheerful lab group. I am greatly grateful for the friendship and support of Kyle Frankel Davis, Michael Saha, Mokganedi Tatlhego, Abi Bhattachan and Zak Ratajczak.

Finally, I thank my great parents Duozhi Li and Hongsheng Yu as well as my sister Chunmei Yu for supporting me throughout all my studies. 


\section{Contents}

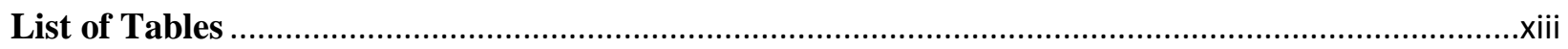

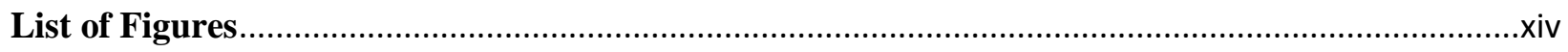

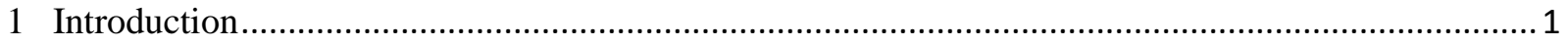

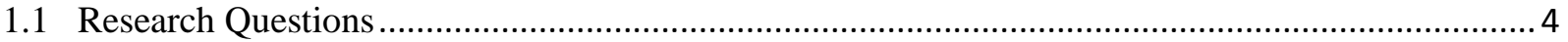

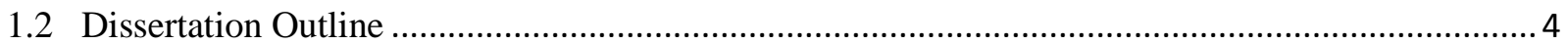

2 An ecohydrological framework for grass displacement by woody plants in savannas.....................

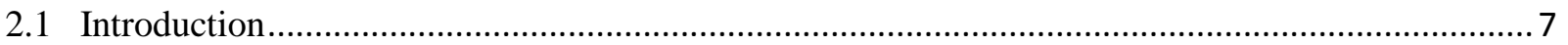

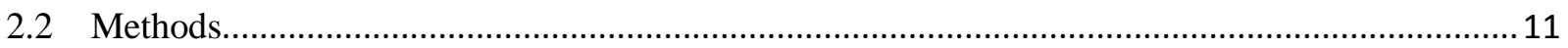

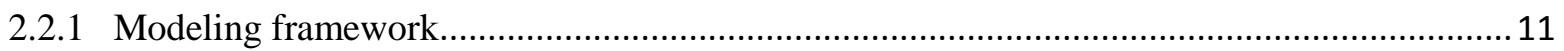

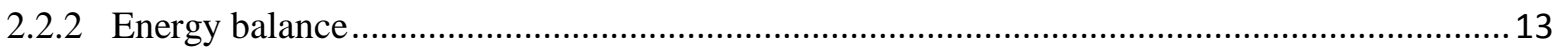

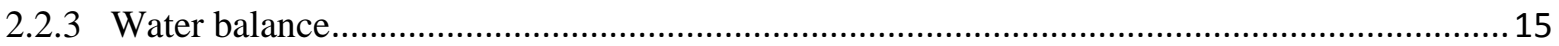

2.2.4 Vegetation dynamics and equilibrium states..................................................................... 19

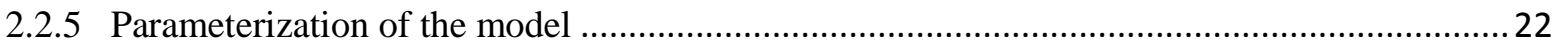

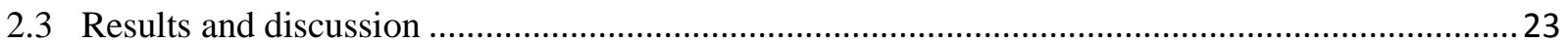

3 Potential of grass invasions in fireproof desert shrublands to create novel ecosystem states under

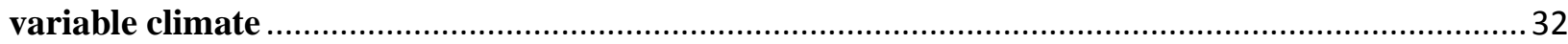

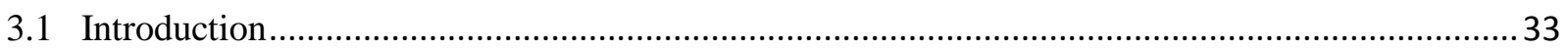

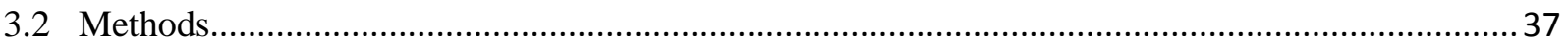

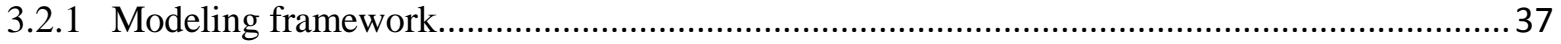

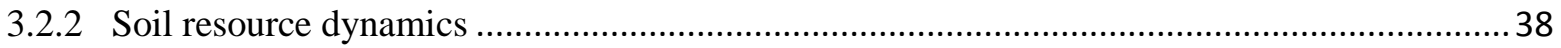

3.2.3 Vegetation dynamics ...............................................................................................

3.2.4 Stochastic dynamics ............................................................................................... 41

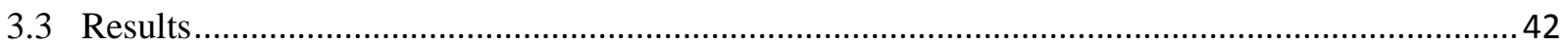

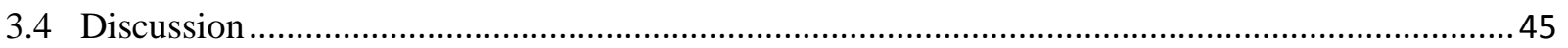

4 Woody plants-grass interactions as affected by hydraulic lift and interannual rainfall variability

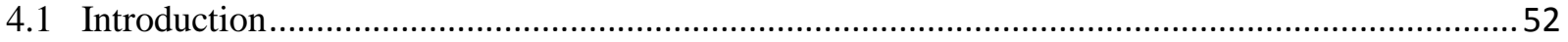

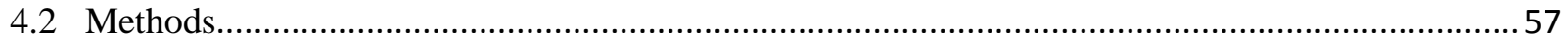

4.2.1 Modelling framework for the role of hydraulic lift...........................................................5 57

4.2.2 Modelling framework for the role of interannual rainfall variability.................................... 60

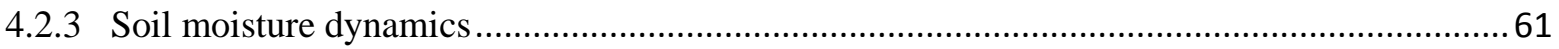


4.2.4 Evapotranspiration by woody plants and grasses........................................................... 62

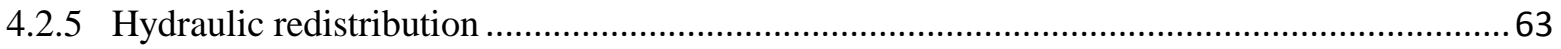

4.2.6 Biomass dynamics of woody plants and grasses.................................................................. 64

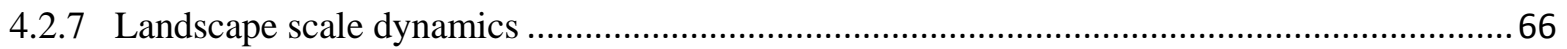

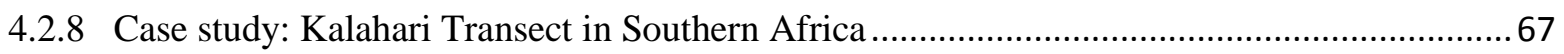

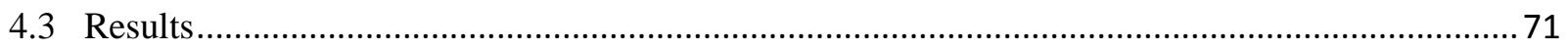

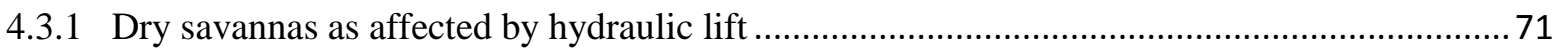

4.3.2 Wet savannas as affected by hydraulic lift ................................................................... 75

4.3.3 Tree-grass composition as affected by interannual rainfall variability .................................. 76

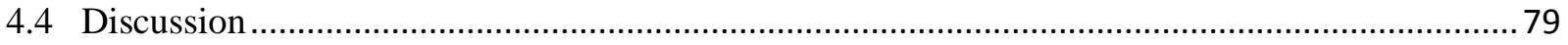

4.4.1 Hydraulic lift as a determinant of tree-grass coexistence on savannas ................................. 79

4.4.2 The effects of interannual rainfall variability on tree-grass composition along Kalahari rainfall

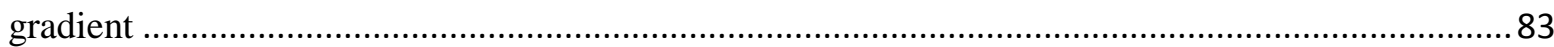

5 Plants with Crassulacean Acid Metabolism outcompete grasses under carbon dioxide enrichment

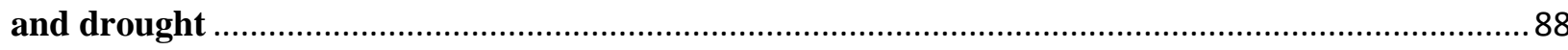

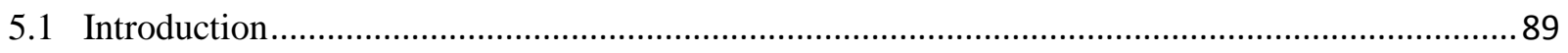

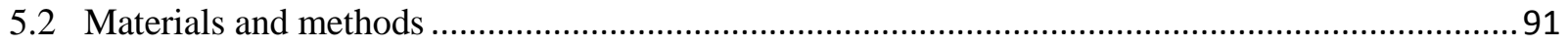

5.2.1 Germination of seeds and growth of seedlings …............................................................ 91

5.2.2 Experimental design and growth chambers ................................................................... 91

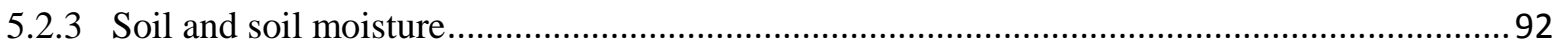

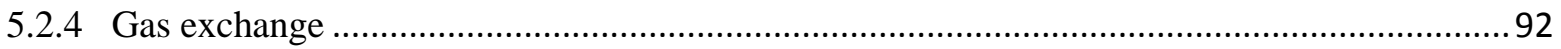

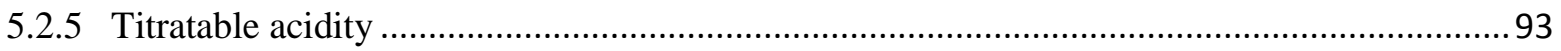

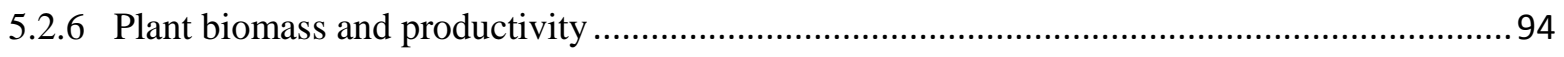

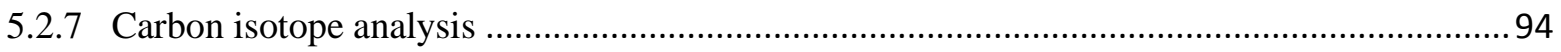

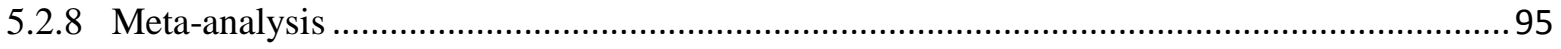

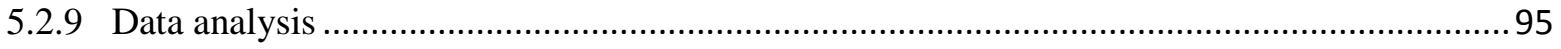

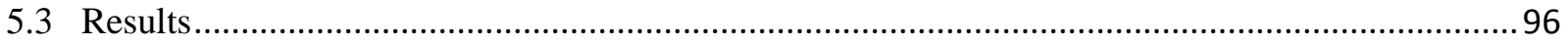

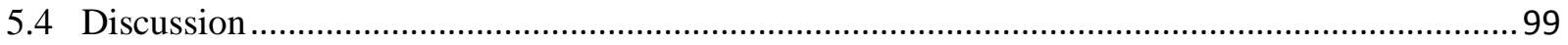

6 Crassulacean acid metabolism in Mesembryanthemum crystallinum: the effects of water stress,

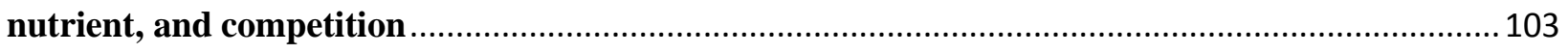

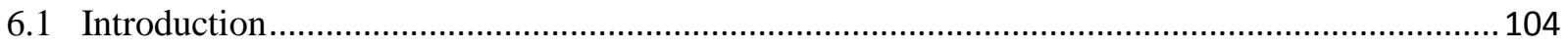

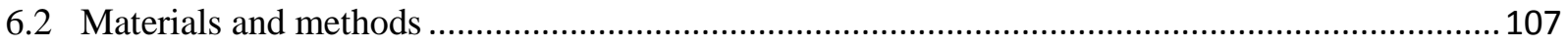

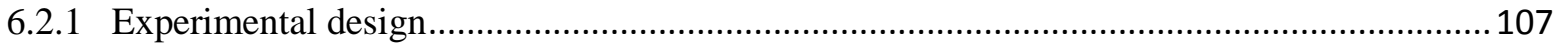

6.2.2 Gas exchange and concentration of titratable acidity .......................................................... 109 
6.2.3 Light availability and biomass measurements ............................................................ 110

6.2.4 Soil moisture, plant leaf water potential and plant $\mathrm{N}$....................................................... 110

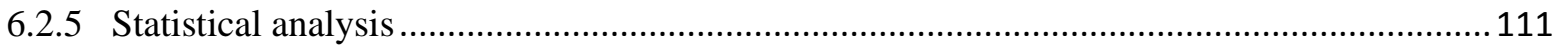

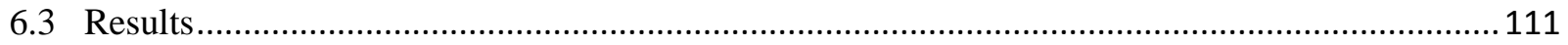

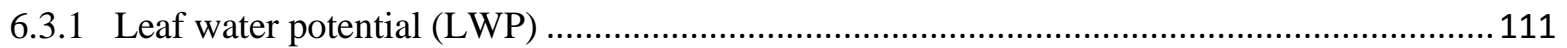

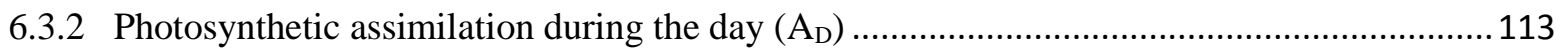

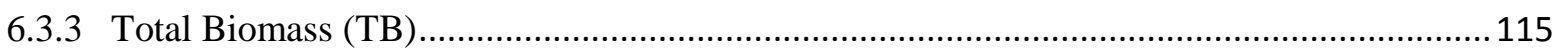

6.3.4 Nocturnal photosynthetic assimilation $\left(\mathrm{A}_{\mathrm{N}}\right)$ and titratable acidity $(\mathrm{TA})$............................117

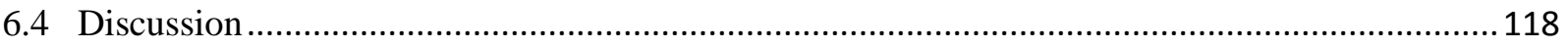

6.4.1 CAM expression and reversibility in M. crystallinum as affected by less intensive droughts

6.4.2 The role of nutrients and their interactions with water availability in the expression and reversibility of CAM assimilation in $M$. crystallinum .............................................................. 120

6.4.3 The effects of nutrient and water conditions on competition between $M$. crystallinum and $B$.

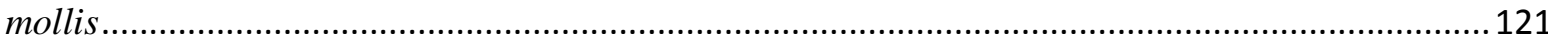

6.4.4 CAM expression and reversibility in M. crystallinum as affected by competition................ 123

7 Hydraulic redistribution in tree-grass, CAM-grass, and tree-CAM associations: the implications

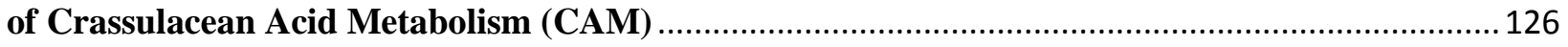

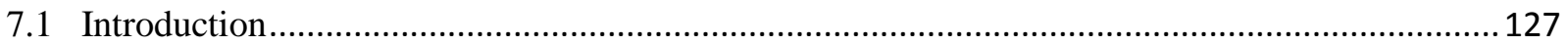

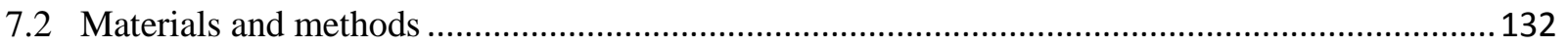

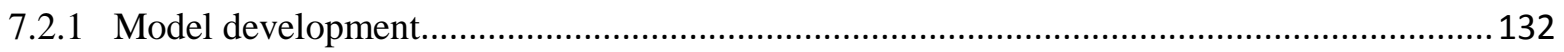

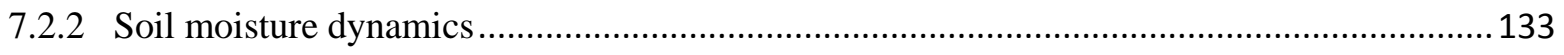

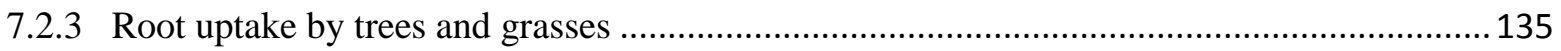

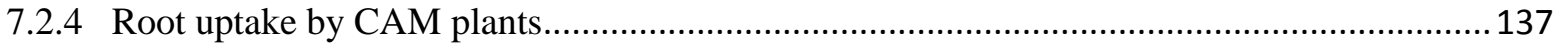

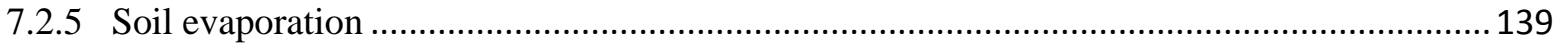

7.2.6 Parameterization of the model …................................................................................... 140

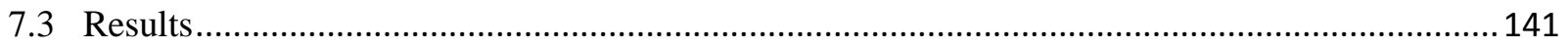

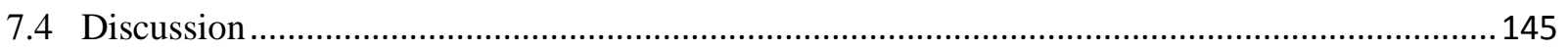

8 Direct and indirect facilitation of plants with Crassulacean Acid Metabolism (CAM) ................151

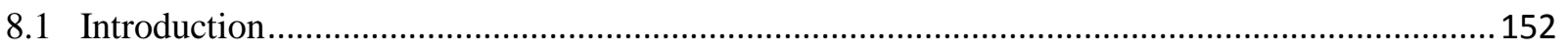

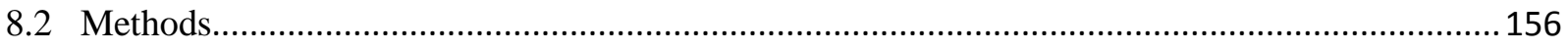

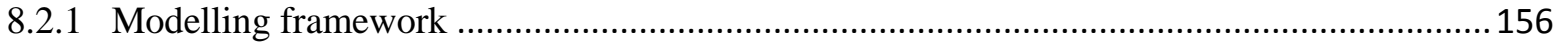

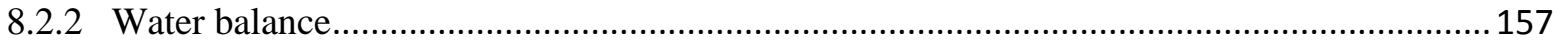

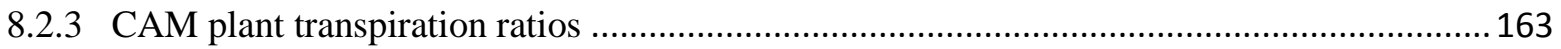




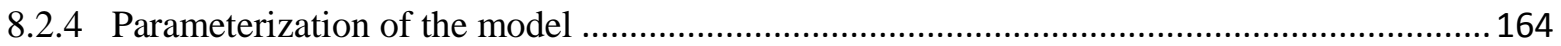

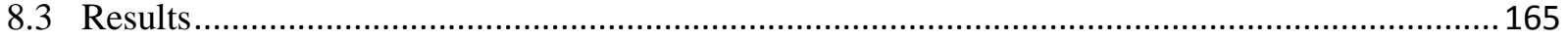

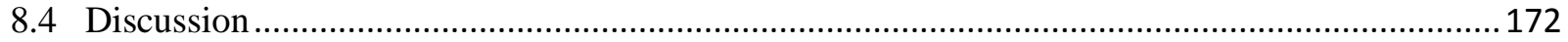

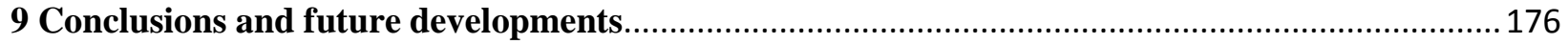

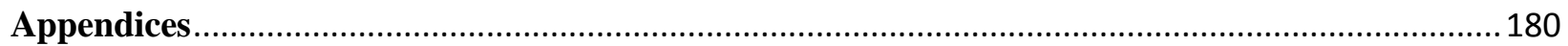

A1 An ecohydrological framework for grass displacement by woody plants in savannas .............. 180

A2 Potential of grass invasions in fireproof desert shrublands to create novel ecosystem states

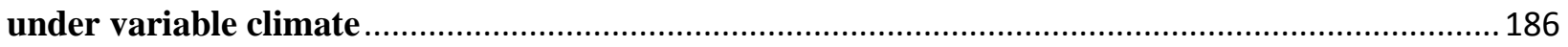

A3 Woody plants-grass interactions as affected by hydraulic lift and interannual rainfall

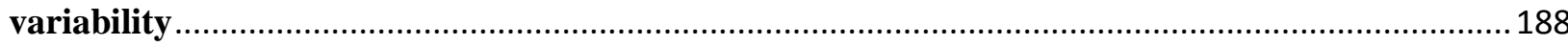

A4 Plants with Crassulacean Acid Metabolism outcompete grasses under carbon dioxide

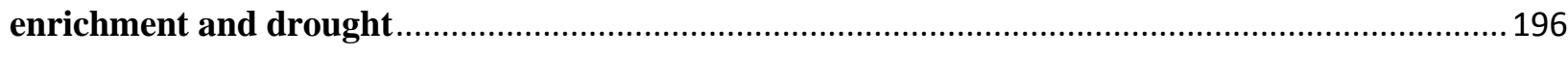
A5 Crassulacean acid metabolism in Mesembryanthemum crystallinum: the effects of water stress,

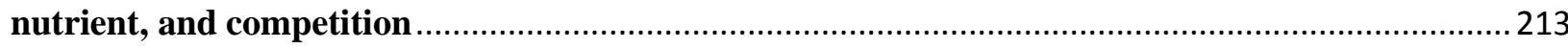

A6 Hydraulic redistribution in tree-grass, CAM-grass, and tree-CAM associations: the implications of Crassulacean Acid Metabolism (CAM) .............................................................. 220

A7 Direct and indirect facilitation of plants with Crassulacean Acid Metabolism (CAM)...........224

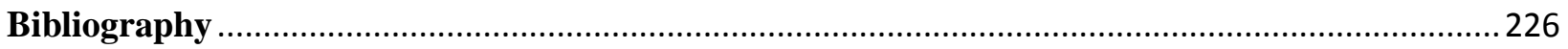




\section{List of Tables}

2.1 A summary of evidence of the occurrence of the fire-vegetation feedback (FVF) and bistability (Bi). 28

7.1 A summary of CAM species which have some deep tap roots and may perform hydraulic redistribution 130

7.2 A summary of vegetation associations in this study .................................................. 132

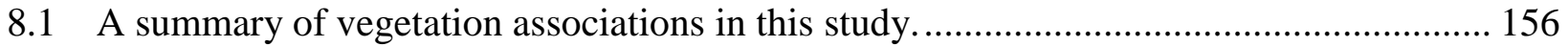

S2.1 Location, elevation (he), mean annual rainfall (MAP), mean atmospheric temperature $\left(\mathrm{Te}_{\mathrm{a}}\right)$ in Tshane and Mongu in Southern African. ........................................................................ 183

S2.2: Parameters, parameter values, and reference sources used in the coupled energy and water

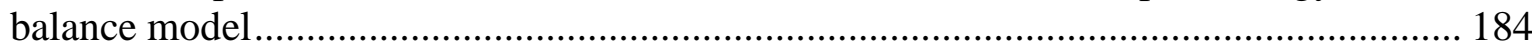

S3.1 A summary of evidence of a high sensitivity of perennial grass to drought ...................... 186

S4.1 A list of abbreviations used to refer to the model variables. .............................................. 188

S4.2 Parameters, parameter values, and reference sources used in the study............................. 190

S4.3 Parameters describing various soil characteristics used in this study................................. 192

S5.1 Results (P values) of five-way factorial ANOVA on total biomass, root/shoot biomass ratio, relative aboveground plant water content (RAWC), and aboveground plant carbon isotope discrimination $(\Delta)$.

S5.2 Observations of vegetation growth in CAM alone (C), grass alone $(\mathrm{G})$, mixture (CM, GM) in each treatment.

S6.1 Results (P values) of five-way factorial ANOVA on total biomass (TB), belowground to aboveground biomass ratio (BA), photosynthetic assimilation during the day (AD), plant leaf water potential (LWP), specific leaf area (SLA), and aboveground plant N (APN) . 213

S7.1 Parameters, parameter values, and reference sources used in the model. 220

S8.1 Parameters, parameter values, and reference sources used in the study. 224

S8.2 Parameters, parameter values, and reference sources used in the study. 225 


\section{List of Figures}

Figure 2-1: Dependence between fire frequency and fuel load (i.e., grass cover) at Kruger National Park, South Africa (experiment data from van Wilgen et al., 2000)................... 20

Figure 2-2: The relationship between woody plant cover $\left(f_{c}\right)$ and grass cover $\left(f_{g}\right)$. Solid and dashed lines represent wet (average rainfall frequency, $\lambda=0.4 \mathrm{~d}^{-1}$ and average rainstorm depth, $\mathrm{h}=11 \mathrm{~mm})$ and dry $\left(\lambda=0.2 \mathrm{~d}^{-1}\right.$ and $\left.\mathrm{h}=11 \mathrm{~mm}\right)$ environment, respectively.

Figure 2-3: Stable (solid black intersection points) and unstable (white intersection point) states of the dynamics obtained as intersection of curves given by equations (5) (solid line) and (6) (dashed line) in two different rainfall regimes: (a) dry (average rain frequency, $\lambda=0.2$ $\mathrm{d}^{-1}$ and average rainstorm depth, $\left.\mathrm{h}=11 \mathrm{~mm}\right)$ environment and $(\mathrm{b})$ wet $\left(\lambda=0.4 \mathrm{~d}^{-1}\right.$ and $\mathrm{h}=$ $11 \mathrm{~mm}$ ) environment.

Figure 2-4: Stable (solid line) and unstable (dashed line) states as a function of mean annual rainfall (MAP). (a) wilting point for grass, $S_{\mathrm{wg}}=0.065$ (thick lines) and $S_{\mathrm{wg}}=0.06$ (fine lines);

Figure 3-1: A conceptual representation of the possible four stages of vegetation transition in desert scrublands as affected by grass invasions.

Figure 3-2: Probability of being in one of three possible states at the final state $(t=3000)$ as a function of $\mathrm{G}$ (shown for the case of one time grass invasion with $\alpha=0.5 ; \beta=1.3 ; \gamma=0.85$; $\mathrm{c} 1=0.08 ; \mathrm{c} 2=2 ; \mathrm{c} 3=0.06 ; \mathrm{c} 4=0.06 ; \mathrm{c} 5=10 ; \mathrm{Fa}=0 ; \sigma \mathrm{S}=0.7 \sigma \mathrm{G})$.

Figure 3-3: Resource concentration, shrub, and grass biomass at the final state ( $\mathrm{t}=3000)$ as a function of $\mathrm{G}$ (shown for the case of one time grass invasion). Same parameters as in Figure 3.2. Resource availability is calculated by equation 1; shrub and grass biomass are calculated as the mean shrub and grass biomass at final state $(\mathrm{t}=3000)$ over 1000 realizations of the stochastic process.

Figure 4-1: A schematic representation of soil water budget during the day (a) and during the night (b). This schematic representation also shows the typical patchy distribution of vegetation with areas covered by woody plant canopies, or "canopy patches" and "intercanopy patches" with sparse grasses and bare soil. The readers can refer to Supplementary Table 4.1 for the meaning of each variable. 58

Figure 4-2: Grassland, savanna, and woodland domains as a function of mean annual precipitation (MAP) and the ratio ( $\alpha 1 / \alpha 2)$ of woody plant root density in the shallow and the deep soil layer. Shading with horizontal lines (i.e., “ $\equiv ”$ indicates the band in the para parameter space in which the existence of savannas is induced by hydraulic lift (HL); in the absence of HL the landscape would be covered by woodland vegetation. Shading with vertical lines (i.e., "||l" indicates the zone of the parameter space where the existence of grasslands is due to hydraulic lift (HL); in the absence of HL savanna would instead exist. (a) Mean rainfall depth, $\mathrm{h}=10 \mathrm{~mm}$ per event; depth of the shallow soil layer, $\mathrm{Z}_{1}=$ $0.25 \mathrm{~m}$; depth of the deep soil layer, $\mathrm{Z}_{2}=0.45 \mathrm{~m}$; soil type: sand. Along line (1): $\mathrm{HL}=$ 0.02-0.04 $\mathrm{mm} \mathrm{d}^{-1}$. Along line (2): $\mathrm{HL}=0.21-0.23 \mathrm{~mm} \mathrm{~d}^{-1}$. (b) Mean rainfall depth, $\mathrm{h}=15$ $\mathrm{mm}$ per event; depth of the shallow soil layer, $\mathrm{Z}_{1}=0.25 \mathrm{~m}$; depth of the deep soil layer, $\mathrm{Z}_{2}$ 
$=0.45 \mathrm{~m}$; soil type: sand. Along line (3): $\mathrm{HL}=0.03-0.06 \mathrm{~mm} \mathrm{~d}^{-1}$. Along line (4): $\mathrm{HL}=$ $0.36-0.37 \mathrm{~mm} \mathrm{~d}^{-1}$. (c) Mean rainfall depth, $\mathrm{h}=10 \mathrm{~mm}$ per event; depth of the shallow soil layer, $Z_{1}=0.25 \mathrm{~m}$; depth of the deep soil layer, $Z_{2}=0.75 \mathrm{~m}$; soil type: sand. Along line (5): $\mathrm{HL}=0.01-0.03 \mathrm{~mm} \mathrm{~d}^{-1}$. Along line (6): $\mathrm{HL}=0.04-0.17 \mathrm{~mm} \mathrm{~d}^{-1}$. (d) Mean rainfall depth, $\mathrm{h}$ $=10 \mathrm{~mm}$ per event; depth of the shallow soil layer, $Z_{1}=0.25 \mathrm{~m}$; depth of the deep soil layer, $\mathrm{Z}_{2}=0.45 \mathrm{~m}$; soil type: sandy loam. Along line (7): $\mathrm{HL}=0.01-0.02 \mathrm{~mm} \mathrm{~d}^{-1}$. Along line (8): $\mathrm{HL}=0.06-0.11 \mathrm{~mm} \mathrm{~d}^{-1}$.

Figure 4-3: Transition between "wet" savanna and woodland vegetation as a function of mean annual precipitation (MAP) and the ratio $(\alpha 1 / \alpha 2)$ between woody root density in the shallow and the deep soil layer with and without hydraulic lift (HL) and fires. Points in the parameter space to the left of these lines correspond to savannas while points to the right correspond to woodlands. (a) Mean rainfall depth, $\mathrm{h}=10 \mathrm{~mm}$ per event; depth of the shallow soil layer, $Z_{1}=0.25 \mathrm{~m}$; depth of the deep soil layer, $Z_{2}=0.45 \mathrm{~m}$; soil type: sand. Along line (1): $\mathrm{HL}=0.13-0.22 \mathrm{~mm} \mathrm{~d}^{-1}$. Along line (2): $\mathrm{HL}=0.23-0.34 \mathrm{~mm} \mathrm{~d}^{-1}$. (b) Mean rainfall depth, $\mathrm{h}=15 \mathrm{~mm}$ per event; depth of the shallow soil layer, $\mathrm{Z}_{1}=0.25 \mathrm{~m}$; depth of the deep soil layer, $\mathrm{Z}_{2}=0.45 \mathrm{~m}$; soil type: sand. Along line (3): $\mathrm{HL}=0.24-0.35 \mathrm{~mm} \mathrm{~d}^{-1}$. Along line (4): $\mathrm{HL}=0.3-0.41 \mathrm{~mm} \mathrm{~d}^{-1}$. The following parameters were used: death rate of woody plants by fires, $\mathrm{k}=0.0002$.

Figure 4-4: (a, b) MODIS-derived tree cover (a) and annual percentage burned (b) as a function of the standard deviation of annual precipitation $\left(\sigma_{\mathrm{AP}}, \mathrm{mm}\right)$ and mean annual rainfall (MAP, mm). MAP is binned into $150 \mathrm{~mm}$ bins and simple linear regression is used to highlight $\sigma_{\mathrm{AP}}$ related trends within these bins.

Figure 4-5: The modeled average grass biomass $(\mathrm{Vg})$ and tree cover $(\mathrm{Fc})$ in alone or associations for the case of sandy soil as affected by interannual rainfall variability (standard deviation of $\lambda, \delta)$ in dry (a, $\lambda=0.2$ event $\mathrm{d}^{-1}, \mathrm{~h}=10 \mathrm{~mm}$ per event) and wet $\left(\mathrm{b}, \lambda=0.4\right.$ event $\mathrm{d}^{-1}, \mathrm{~h}=$ $10 \mathrm{~mm}$ per event) environments. 78

Figure 5-1: Total biomass (TB, g) of Cylindropuntia imbricata (CAM) and Bouteloua eriopoda $\left(\mathrm{C}_{4}\right.$ grass) in $\mathrm{CAM}$ alone $(\mathrm{C})$, grass alone $(\mathrm{G})$, mixture $(\mathrm{CM}, \mathrm{GM})$ in the first stage (a, November 13th 2015) and the second stage (b, December 23th 2015) in each treatment. $\mathrm{HCHW}$ refers to high $\mathrm{CO}_{2}$ and well-watered conditions; HCLW refers to high $\mathrm{CO}_{2}$ and drought conditions; LCHW refers to low $\mathrm{CO}_{2}$ and well-watered conditions; LCLW refers to low $\mathrm{CO}_{2}$ and drought conditions. The error bars represent $95 \%$ confidence intervals... 96

Figure 5-2: Diurnial change of rates of carbon dioxide uptake (A, umol m $\left.\mathrm{m}^{-2} \mathrm{~s}^{-1}\right)$ and titratable acidity $\left(\mathrm{mmol} \mathrm{m}^{-2}\right)$ by Cylindropuntia imbricata in well-watered $(\mathrm{a}, \mathrm{b})$ and drought $(\mathrm{c}, \mathrm{d})$ conditions. $C$. imbricata performs $\mathrm{C}_{4}$ photosynthesis at night and $\mathrm{C}_{3}$ photosynthesis in the late afternoon, as shown by tritiable acidicity measurements. Values are mean \pm standard deviation $(\mathrm{n}=9)$.

Figure 5-3: A conceptual representation of growth responses of CAM alone (C), grass alone (G), CAM-grass associations ( $\mathrm{CM}, \mathrm{GM})$ to $\mathrm{CO} 2$ and water manupilations. $\mathrm{H}$ refers to high while $\mathrm{L}$ refers to low. The panel in the right and above indicates the initial stage of plants before $\mathrm{CO}_{2}$ and water treatments. Green color indicates that plants are alive while brown color indicates that plants are dead. 
Figure 6-1: Schematic diagram of water treatments in high and low water conditions. Black zone represents low frequency watering treatment (once in every 8 days) while the white zone represents high frequency watering treatment (once in every 2 days). Plants were harvested in the first (Oct 30-31th), second (Dec 4-5th), and third (Jan 4-5th) stages of the experiment. Gas exchange and titratable acidity were measured 1-2 days before each harvest.

Figure 6-2: Leaf water potential (LWP) in Mesembryanthemum crystallinum alone (FC), Mesembryanthemum crystallinum in mixture (FCM), Bromus mollis alone (G), Bromus mollis in mixture (GM) under different nutrient and water conditions in the first (a), second (b), an and third (c) stages of the experiment. HNHW refers to high nutrient and high water conditions; LNHW refers to low nutrient and high water conditions; HNLW refers to high nutrient and low water conditions; LNLW refers to low nutrient and low water conditions. Each bar represents the mean of 6 values while error bar indicates $95 \%$ confidence intervals. 112

Figure 6-3: Photosynthetic assimilation during the day (AD) in Mesembryanthemum crystallinum alone (FC), Mesembryanthemum crystallinum in mixture with B. mollis (FCM), Bromus mollis alone $(\mathrm{G})$, Bromus mollis in mixture with M. crystallinum (GM) under different nutrient and water conditions in the first (a), second (b), and third (c) stages of the experiment. Symbols for each treatment are the same as Figure 6.2. Each bar represents the mean of 6 values while error bar indicates $95 \%$ confidence intervals. 114

Figure 6-4: Total biomass (TB) in Mesembryanthemum crystallinum alone (FC), Mesembryanthemum crystallinum in mixture (FCM), Bromus mollis alone (G), Bromus mollis in mixture (GM) under different nutrient and water conditions in the first (a), second (b), and third (c) stages of the experiment. Symbols for each treatment are the same as Figure 6.2. Each bar represents the mean of 6 values while error bar indicates $95 \%$ confidence intervals. 116

Figure 6-5: Nocturnal change of photosynthetic assimilation (AN) of Mesembryanthemum crystallinum in alone (FC) and mixture (FCM) in low water conditions in the second stage of the experiment. Symbols for each treatment are the same as in Figure 6.2. (b) Titratable acidity (TA) of Mesembryanthemum crystallinum in alone (FC) and mixture (FCM) in low water conditions in the second stage. 6 refers to 6 pm while 8 refers 8 am. Each bar represents the mean of 6 values while error bar indicates $95 \%$ confidence intervals. Both FC and FCM do not express CAM behavior in any treatments during the first and third stages of the experiment, and thus values of $A_{N}$ and TA in these two stages are not shown.

Figure 7-1: Schematic diagram of water flux within canopies of CAM plants. $\Psi_{1}$ : leaf water potential; $\Psi_{\mathrm{s}}$ : soil water potential; $\Psi \mathrm{x}$ : xylem water potential; $\Psi \mathrm{w}$ : plant storage water potential; f: fraction of plant resistance below the storage branch connection; $\mathrm{g}_{\mathrm{p}}$ plant conductance per unit leaf area $\left(r_{p}\right.$, plant resistance per unit leaf area); $\mathrm{g}_{\mathrm{c}}$ storage conductance per unit leaf area; $D_{\max }$ maximum depth of water per unit leaf area; $\mathrm{D}_{\mathrm{w}}$ actual depth of water per unit leaf area. Adapted from Lhomme et al (2001) and Bartlett et al (2014). 
Figure 7-2: Hydraulic redistribution (HR) as affected by different types of vegetation associations with $\mathrm{h}=10 \mathrm{~mm}, \lambda=0.1 \mathrm{~d}^{-1}$ (a) and $\lambda=0.2 \mathrm{~d}^{-1}(\mathrm{~b})$ for the case of a high mesophyll conductance $\left(\mathrm{g}_{\mathrm{m}}=0.003 \mathrm{~m} \mathrm{~s}^{-1}\right)$. T-G refers to Tree-Grass association; $\mathrm{CH}-\mathrm{G}$ refers to CAM-Grass association with a relatively high value of hydraulic conductivity; CL-G refers to CAM-Grass association with a relatively low value of hydraulic conductivity; T-C refers to Tree-CAM association.

Figure 7-3: $(\mathrm{a}, \mathrm{b})$ Actual evapotranspiration components as affected by different types of vegetation associations with $\mathrm{h}=10 \mathrm{~mm}, \lambda=0.1 \mathrm{~d}^{-1}$ (a) and $\lambda=0.2 \mathrm{~d}^{-1}$ (b) for the case of a high mesophyll conductance $\left(\mathrm{g}_{\mathrm{m}}=0.003 \mathrm{~m} \mathrm{~s}^{-1}\right)$. $\mathrm{T}_{2}$ refers to transpiration in the deep soil layer; $\mathrm{T}_{1 \mathrm{~d}}$ refers to transpiration in the shallow soil layer by deep-rooted plants; $\mathrm{T}_{1 \mathrm{~s}}$ refers to transpiration in the shallow soil layer by shallow-rooted plants; E refers to evaporation at soil surface. $(\mathrm{c}, \mathrm{d})$ The ratio of actual evapotranspiration in the shallow soil layer $\left(\mathrm{ET}_{1}=\right.$ $\left.\mathrm{T}_{1 \mathrm{~d}}+\mathrm{T}_{1 \mathrm{~s}}+\mathrm{E}\right)$ to transpiration in the deep soil layer $\left(\mathrm{T}_{2}\right)$ as affected by different types of vegetation associations with $\mathrm{h}=10 \mathrm{~mm}, \lambda=0.1 \mathrm{~d}^{-1}(\mathrm{c})$ and $\lambda=0.2 \mathrm{~d}^{-1}(\mathrm{~d})$ 144

Figure 8-1: Schematic diagram of indirect (dotted line) and direct (solid line) interactions among C3 trees, C4 grasses, and CAM plants. $(a, b)$ trees suppress grass transpiration through solar radiation reduction (SRR) and reduce the competitive effect of grasses on CAM plants in access to soil water resources, thus indirectly facilitating CAM plants. (a) Trees and CAM plants compete for soil water resources because of a high degree of root overlap; (b) trees directly facilitate CAM plants in situations of a low to moderate root overlap. (c) Inclusion of grasses in tree-CAM associations increases hydraulic lift suppressing trees and thus may indirectly facilitate CAM plants.

Figure 8-2: Transpiration ratio ( $\xi$ ) of CAM plants between CAM associations and CAM alone for $\operatorname{arid}\left(\lambda=0.2 \mathrm{~d}^{-1}\right.$ and $\left.\mathrm{h}=5 \mathrm{~mm}\right)$ (a) and semiarid $\lambda=0.2 \mathrm{~d}^{-1}$ and $\mathrm{h}=10 \mathrm{~mm}$ ) (b) environment in loamy sand in the case of a high degree of root overlap between trees and CAM plants. Parameters: the depth of shallow soil layer, $Z_{1}=10 \mathrm{~cm}$; the depth of deep soil layer, $\mathrm{Z}_{2}=10 \mathrm{~cm}$; grass cover in arid and semiarid environment, $\mathrm{f}_{\mathrm{g}}=70 \%$; root allocation into the deep soil layer, $\mathrm{r}_{2} / \mathrm{r}_{1}=0.2$.

Figure 8-3: Actual evapotranspiration components in the shallow soil layer for CAM plants alone (C), tree-CAM (T-C), tree-CAM-grass (T-C-G), and CAM-grass (C-G) associations for arid $\left(\lambda=0.2 \mathrm{~d}^{-1}\right.$ and $\left.\mathrm{h}=5 \mathrm{~mm}\right)(\mathrm{a})$ and semiarid $\left(\lambda=0.2 \mathrm{~d}^{-1}\right.$ and $\left.\mathrm{h}=10 \mathrm{~mm}\right)(\mathrm{b})$ environment in loamy sand in the case of a high degree of root overlap between trees and CAM plants. $\mathrm{T}_{1 \mathrm{t}}$ refers to the transpiration by trees, $\mathrm{T}_{1 \mathrm{~g}}$ refers to the transpiration by grasses, $\mathrm{T}_{1 \mathrm{c}}$ refers to the transpiration by CAM plants, and $\mathrm{E}$ refers to evaporation from the soil surface. Parameters: the same as Figure 3 (a, b). (c, d) Actual evapotranspiration components in the shallow soil layer for CAM plants alone $(\mathrm{C})$, tree-CAM (T-C), treeCAM-grass (T-C-G), and CAM-grass (C-G) associations for arid $\left(\lambda=0.2 \mathrm{~d}^{-1}\right.$ and $\mathrm{h}=5$ $\mathrm{mm})(\mathrm{c})$ and semiarid $\left(\lambda=0.2 \mathrm{~d}^{-1}\right.$ and $\left.\mathrm{h}=10 \mathrm{~mm}\right)(\mathrm{d})$ environment, in loamy sand in the case of a low to moderate root overlap between trees and CAM plants. $\mathrm{r}_{2} / \mathrm{r}_{1}$ : root allocation into the deep soil layer. The number " 3 " means $r_{2} / r_{1}=3$ while the number " 1 " means $r_{2} / r_{1}$ $=1$. Parameters: depth of shallow soil layer, $Z_{1}=10 \mathrm{~cm}$; depth of deep soil layer, $Z_{2}=30$ $\mathrm{cm}$; grass cover in arid and semiarid environment, $\mathrm{f}_{\mathrm{g}}=70 \%$.

Figure 8-4: Hydraulic redistribution (HR) by trees in tree-Cam (T-C) and tree-CAM-grass (T-C$\mathrm{G})$ associations for arid $\left(\lambda=0.2 \mathrm{~d}^{-1}\right.$ and $\left.\mathrm{h}=5 \mathrm{~mm}\right)$ (a) and semiarid $\left(\lambda=0.2 \mathrm{~d}^{-1}\right.$ and $\mathrm{h}=10$ 
mm) (b) environments. (c, d) Transpiration ratio $(\xi)$ of CAM plants between CAM associations and CAM alone for arid $\left(\lambda=0.2 \mathrm{~d}^{-1}\right.$ and $\left.\mathrm{h}=5 \mathrm{~mm}\right)(\mathrm{d})$ and semiarid $(\lambda=0.2$ $\mathrm{d}^{-1}$ and $\left.\mathrm{h}=10 \mathrm{~mm}\right)(\mathrm{d})$ environments. All panels refer to the case of loamy sand and low to moderate root overlap between trees and CAM plants. Parameters: the same as Figure 3 (c, d). 169

Figure 8-5: Transpiration ratio $(\xi)$ of CAM plants between tree-CAM-grass association (T-C-G) and tree-CAM association (T-C) for arid $\left(\lambda=0.2 \mathrm{~d}^{-1}\right.$ and $\left.\mathrm{h}=5 \mathrm{~mm}\right)$ (a) and semiarid $(\lambda=$ $\left.0.2 \mathrm{~d}^{-1}\right)$ and $\left.\mathrm{h}=10 \mathrm{~mm}\right)(\mathrm{b})$ environments in loamy sand as affected by grass cover $\left(\mathrm{f}_{\mathrm{g}}\right)$. (c, d) Hydraulic redistribution $(H R)$ and grass transpiration $\left(\mathrm{T}_{\mathrm{g}}\right)$ in tree-CAM-grass $(\mathrm{T}-\mathrm{C}-\mathrm{G})$ association in loamy sand as affected by grass cover $\left(f_{g}\right)$ for arid (c) and semiarid $(d)$ environments. Parameters: the depth of shallow soil layer, $Z_{1}=10 \mathrm{~cm}$; the depth of deep soil layer, $Z_{2}=30 \mathrm{~cm}$.

Supplementary Figure 2-1: The relationship between maximum woody plant cover (fcmax) and mean annual rainfall (MAP) in African savannas (modified from Sankaran et al., 2005).

Supplementary Figure 2-2: The relationship between ratio of fire-induced mortality to growth rate of woody plants $(\mathrm{d} / \mathrm{b})$ and rainfall during the growing season.

Supplementary Figure 2-3: Dependence between fire frequency and fuel load (i.e., grass cover) at Kruger National Park, South Africa (experiment data from van Wilgen et al., 2000).. 182

Supplementary Figure 4-1: Dependence of woody plant fractional cover (fc) and landscape scale hydraulic lift (HL) on mean annual precipitation (MAP) at steady states. The following parameters were used: mean rainfall depth, $\mathrm{h}=10 \mathrm{~mm}$ per event; root density of woody plants in the deep soil layer, $\alpha 2=0.15$. The arrow $(\downarrow)$ indicates the MAP at which the fractional root cover of woody plants (at the landscape scale) is, $f_{r}>0.95$.

Supplementary Figure 4-2: The effects of the ratio $\left(\alpha_{1} / \alpha_{2}\right)$ of woody root density in the shallow and deep soil layer on the fraction $(\gamma)$ of woody plants biomass relying on the shallow soil layer. The lines of (1), (2), (3), (4), (5), 6), 7), and (8) correspond to the the lines (1), (2), (3), (4), (5), 6), (7), and (8) in Figure 4-2 (i.e., in the presence of hydraulic lift (HL)).

Supplementary Figure 4-3: The effects rainfall frequency $(\lambda)$ and mean annual precipitation (MAP) on drainage $\left(\mathrm{D}_{2}\right)$ from the deep soil layer (i.e., from beneath the root zone of woody plants). The lines of (2), (4), (6) and (8) correspond to the lines of (2), (4), (6) and (8) in Figure 4-2 (i.e., in the presence of hydraulic lift (HL)).

Supplementary Figure 5-1: Observations of C. imbricata (CAM) and B. eriopoda (C 4 grass) in Sevilleta National Wildlife Refuge in 1993 (A) and 2015 (B). 200

Supplementary Figure 5-2: Observations of $C$. imbricata which are stems with cylindrical shape. 201

Supplementary Figure 5-3: Relative abovegorund plant water content (RAWC, \%) of $C$. imbricata $(\mathrm{CAM})$ and B. eriopoda ( $\mathrm{C}_{4}$ grass) in CAM alone $(\mathrm{C})$, grass alone $(\mathrm{G})$, mixture (CM, GM) in the first stage (a, November 13th 2015) and the second stage (b, December 23th 2015) in each treamtment. HCHW refers to high CO2 and well-watered conditions; 
HCLW refers to high $\mathrm{CO}_{2}$ and drought conditions; LCHW refers to low $\mathrm{CO}_{2}$ and wellwatered conditions; LCLW refers to low $\mathrm{CO}_{2}$ and drought conditions. The error bar indicates $95 \%$ confidence intervals 202

Supplementary Figure 5-4: Growth rate (the total biomass ratio between the first and the second stage) of $\mathrm{C}$. imbricata (CAM) and B. eriopoda ( $\mathrm{C}_{4}$ grass) in CAM alone $(\mathrm{C})$, grass alone $(\mathrm{G})$, mixture (CM, GM) in each treatment. Symbols for each treatment are the same as Supplementary Figure 5.3. The error bar indicates $95 \%$ confidence intervals. 203

Supplementary Figure 5-5: Soil moisture content (S, \%) in CAM alone (C), grass alone (G), mixture (M) in the first stage (a, November 13th 2015) and the second stage (b, December 23th 2015) in each treatment. Symbols for each treatment are the same as Supplementary Figure 5.3. The error bar indicates $95 \%$ confidence intervals. 204

Supplementary Figure 5-6: Aboveground plant carbon isotope discrimination $(\Delta)$ of $C$. imbricata $(\mathrm{CAM})$ and B. eriopoda $\left(\mathrm{C}_{4}\right.$ grass) in CAM alone $(\mathrm{C})$, grass alone $(\mathrm{G})$, mixture $(\mathrm{CM}, \mathrm{GM})$ in the first stage (a, Novenber 13th 2015) and the second stage (b, December 23th 2015) in each treamtment. Symbols for each treatment are the same as Supplementary Figure 5.3. Each bar represents the mean of 6 values while error bar indicates 95\% confidence intervals. 205

Supplementary Figure 5-7: Root/shoot ratio (BA) of C. imbricata $(\mathrm{CAM})$ and B. eriopoda $\left(\mathrm{C}_{4}\right.$ grass) in CAM alone $(C)$, grass alone $(\mathrm{G})$, mixture $(\mathrm{CM}, \mathrm{GM})$ in the first stage (a, November 13th 2015) and the second stage (b, December 23th 2015) in each treatment. Symbols for each treatment are the same as Supplementary Figure 5.3. The error bars represent $95 \%$ confidence intervals 206

Supplementary Figure 5-8: Meta-analysis of log ratio of daily $\mathrm{CO}_{2}$ uptake (a) and biomass (b) under elevated $\mathrm{CO}_{2}$ concentrations to ambient $\mathrm{CO}_{2}$ concentrations for different families of consititude CAM plants, including 'Agavaceae', 'Bromeliaceae', 'Cactaceae', 'Crassulaceae', 'Orchidaceae'. 207

Supplementary Figure 6-1: Gravimetrically soil moisture (S) in CAM alone (FC), grass alone $(\mathrm{G})$, and mixture $(\mathrm{M})$ as affected by nutrient and water conditions in the first (a), second (b), and third (c) stages. HNHW refers to high nutrient and high water conditions; LNHW refers to low nutrient and high water conditions; HNLW refers to high nutrient and low water conditions; LNLW refers to low nutrient and low water conditions. Each bar represents the mean of 6 values while error bar indicates 95\% confidence intervals....... 216

Supplementary Figure 6-2 Specific leaf area (SLA) in CAM alone (FC), CAM in mixture $(\mathrm{FCM})$, grass alone $(\mathrm{G})$, grass in mixture $(\mathrm{GM})$ as affected by nutrient and water conditions in the first (a), second (b), and third (c) stages. Symbols for each treatment are the same as Supplementary Figure 6.1. Each bar represents the mean of 6 values while error bar indicates $95 \%$ confidence intervals.

Supplementary Figure 6-3: Relative light intensity (RLI) for CAM in mixture as affected by nutrient and water conditions in the first, second, and third stages. Symbols for each treatment are the same as Supplementary Figure 6.1. Each bar represents the mean of 6 values while error bar indicates $95 \%$ confidence intervals. 
Supplementary Figure 6-4: Aboveground plant nitrogen (APN) in CAM alone (FC), CAM in mixture (FCM), grass alone $(\mathrm{G})$, grass in mixture $(\mathrm{GM})$ as affected by nutrient and water conditions in the first (a), second (b), and third (c) stages. Symbols for each treatment are the same as Supplementary Figure 6.1. Each bar represents the mean of 6 values while error bar indicates $95 \%$ confidence intervals.

Supplementary Figure 7-1: Hydraulic redistribution (HR) as affected by different types of vegetation associations with $\mathrm{h}=10 \mathrm{~mm}, \lambda=0.1 \mathrm{~d}^{-1}$ (a) and $\lambda=0.2 \mathrm{~d}^{-1}$ (b) for the case of a low mesophyll conductance $\left(g_{m}=0.0015 \mathrm{~m} \mathrm{~s}^{-1}\right)$. The symbols are the same as Figure 7.2 $(a, b)$ in the main text.

Supplementary Figure 7-2: (a, b) Actual transpiration by deep-root plants $\left(\mathrm{T}_{\mathrm{d}}\right)(\mathrm{a})$ and shallowrooted plants $\left(\mathrm{T}_{\mathrm{s}}\right)(\mathrm{b})$ as affected by different types of vegetation associations for sandy loam with $\lambda=0.1 \mathrm{~d}-1, \mathrm{~h}=10 \mathrm{~mm}$. (c, d) Actual transpiration by deep-root plants $\left(\mathrm{T}_{\mathrm{d}}\right)$ and shallowed-rooted plants $\left(\mathrm{T}_{\mathrm{s}}\right)(\mathrm{d})$ as affected by different types of vegetation associations with $\lambda=0.2 \mathrm{~d}^{-1}, \mathrm{~h}=10 \mathrm{~mm}$ for sandy loam. These results are for the case of a high mesophyll conductance $\left(\mathrm{g}_{\mathrm{m}}=0.003 \mathrm{~m} \mathrm{~s}^{-1}\right)$. 223 


\section{Introduction}

Drylands are regions where precipitation is typically low relative to evapotranspiration and therefore the ecosystem productivity is water limited. If we include regions in arid, semiarid, and dry-subhumid climates, drylands make up about $40 \%$ of the terrestrial land surface and support at least 2 billion people (Millennium Ecosystem Assessment, 2005). Global environmental changes resulting from anthropogenic activities have led to changes in the concentrations of $\mathrm{CO}_{2}$ and other trace gases in the atmosphere (Vitousek et al., 1997; Karl and Trenberth, 2003). Global climate change studies predict a more frequent occurrence of extreme rainfall events, with increased risks of drought and intense precipitation events, and an overall enhanced rainfall variability (e.g., Easterling et al., 2000), especially in drylands (IPCC, 2014). These changes in environmental conditions have led to significant changes in vegetation cover and plant community composition, with important impacts on ecohydrological (Huxman et al., 2005) and geochemical (Hibbard et al., 2001) processes, regional climate (e.g., Zeng et al., 1999; He et al., 2014) and the provision of ecosystem services such as livestock grazing, soil erosion prevention, and carbon sequestration.

Some of the major changes in dryland vegetation are associated with shifts in woody plantgrass interactions. For instance, the encroachment of woody plants into grasslands has been widely documented in many regions worldwide (Archer et al., 1995; Van Auken, 2000; Moleele et al., 2002). This phenomenon typically entails either the transition to a savanna state with a mixed community of grasses and woody plants, or - more commonly - the replacement of the grasses with woody plants, often occurring in scattered vegetated patches bordered by bare soil (e.g., Schlesinger et al., 1990; Archer et al., 1995). The transition from grassland to woodland has been associated with exogenous drivers (i.e., overgrazing, fire suppression, increase in $\mathrm{CO}_{2}$ 
concentration) and endogenous factors (positive feedbacks between vegetation and soil erosion or fire regime) (D'Odorico et al., 2012). While the increase in grazer density and the consequent reduction of grass cover and fire pressure are often invoked to explain woody plant encroachment (Anderies et al., 2002; D’Odorico et al., 2006a; Okin et al., 2009a), it remains unclear whether woody plant encroachment itself can reduce grass cover without grazing.

Another recurrent type of change in dryland vegetation is the invasion of desert shrublands by exotic annual and perennial grasses. Grass invasions typically increase fire frequency and intensity and thus increase the mortality rates of shrubland species, particularly if they are not adapted to fire (e.g., Bond et al., 2005; Runyan et al., 2012). The loss of shrub biomass further enhances the establishment and spread of invasive grasses (D'Antonio, 2000). Known as "the fire cycle" (D'Antonio and Vitousek, 1992), this positive feedback leads to the replacement of fireintolerant native shrubs with exotic grasses. However, it is less clear whether grass invasions could interact with fire dynamics in the presence of increased rainfall variability to convert these once fire-proof shrublands into highly degraded barren landscapes.

In addition to these "win-loss" scenarios associated with woody plant-grass interactions (i.e., shrub encroachment or grass invasion), some changes in vegetation dynamics do not necessarily result in the loss of either one of these two plant functional types. For instance, savanna ecosystems exhibit a stable coexistence of woody plants and grasses; they occur in many regions of the world, including Africa, Australia, South and North America, and Europe (Scholes and Walker, 1993; Scholes and Archer, 1997). Known as the "savanna question" (Sarmiento, 1984), the enigma of tree-grass coexistence has often been explained either by invoking spatial (Walter, 1971; Walker and Noy-Meir, 1982; Eagleson and Segarra, 1985) or temporal (Scholes and Archer, 1997; House et al., 2003) niche separation between the two functional groups 
(equilibrium theories), or by challenging the notion that savannas are systems in a stable equilibrium (disequilibrium theories) (Sankaran, 2004). It remains unclear, however, whether facilitation mechanisms associated, for instance, with hydraulic lift could also contribute to woody plant-grass coexistence. In fact, HL is expected to favor grasses and/or tree seedlings, which are found to be capable of scavenging the water lifted by trees (Dawson, 1993; Zou et al., 2005; Scott et al., 2008; Quijano et al., 2012).

Unlike changes in the dryland vegetation associated with woody plant-grass interactions, an often overlooked phenomenon is the increase in the abundance of plants which conduct Crassulacean Acid Metabolism (CAM). CAM plants perform a temporal separation of the light and dark reactions of photosynthesis (Lüttge, 2004; Borland et al., 2011) and their water storage and photosynthetic plasticity can buffer fluctuations in environmental conditions (Borland et al., 2009, 2011), which provides CAM plants with ecological opportunities to increase their abundance under drier climate conditions (Drennan and Nobel, 2000; Cushaman and Borland, 2002; Borland et al., 2009; Reyes-García and Andrade, 2009). In fact, in the last few decades research in ecology has investigated the response of $\mathrm{C}_{3}$ and $\mathrm{C}_{4}$ plants to global environment change (e.g., Smith, 2011; D’Odorico et al., 2012; Higgins and Scheiter, 2012; Kulmatiski and Beard, 2013a). However, an integrated evaluation of the response of CAM plants and their competitive relationships with other functional groups (i.e., $\mathrm{C}_{3}$ and $\mathrm{C}_{4}$ plants) to global environment change (i.e., $\mathrm{CO}_{2}$ enrichment, drought, $\mathrm{N}$ deposition) is still missing. The expansion of CAM plants in drylands could bring new opportunities for the use of marginal lands and enhance human adaptation to climate change by promoting bioenergy production and carbon sequestration (e.g., Borland et al., 2015; Owen et al., 2015). Therefore, understanding the potential shift in the competitive relationships between CAM plants and other functional groups 
is crucial for evaluations of ecosystem resilience and productivity, and the provision of ecosystem services under global environmental change.

\subsection{Research Questions}

The broad aim of this dissertation is to provide new insights into ecohydrological controls of two major changes in dryland vegetation associated with woody plants-grass interactions and expansion of CAM plants under global environmental change. To this end, new mechanistic models integrated with field and satellite data and empirical experiments are developed to address the following research questions:

i) how does woody plant encroachment suppress grass production even in the absence of grazing and to what extent can fire-vegetation feedbacks lead to bistable dynamics in grasslands-shrubland transition zones?

ii) how do grass invasions and climate change (increased rainfall fluctuations) act in concert to induce the transition from shurbland to unvegetated conditions?

iii) can hydraulic lift be invoked as a mechanism responsible for woody plant-grass coexistence in savannas?

iv) how do major drivers of global environmental change (i.e., $\mathrm{CO}_{2}$ enrichment, drought, $\mathrm{N}$ deposition) affect the competitive relationship between CAM plants and other functional groups (i.e., $\mathrm{C}_{3}$ and $\mathrm{C}_{4}$ plants)?

\subsection{Dissertation Outline}

The dissertation is divided into 9 chapters. Following the introduction in chapter 1, chapter 2 describes an ecohydrological framework for the study of the dependence of grass density on woody plant cover. It investigates the interaction of woody plants and grasses with soil water and 
light through a coupled energy and water balance model accounting for the competitive advantage of woody plants over grasses due to lateral root spread and woody plant shading. In Chapter 3, a stochastic process-based model of vegetation-resource dynamics was used to investigate the effect of grass invasions on the temporal dynamics of desert shrublands in a randomly fluctuating environment. This model accounted for the interactions between shrubs and grasses, and for their coupling with the dynamics of soil resources. Chapter 4 develops a mechanistic model to investigate the role of hydraulic redistribution (HR) on the interactions between woody plants and grasses and the dynamics of savanna ecosystems. The model uses a stochastic representation of rainfall and soil moisture dynamics and deterministic laws to express the interactions and dynamics of woody plants and grasses. While the model does not explicitly represent the spatial interactions among woody plants and grasses, it accounts for the impact on the soil water balance of spatial heterogeneities associated with the mosaic-like structure of the landscape and uses a lumped approach to relate patch-scale to landscape-scale variables. In this chapter, this model was further adapted to investigate the response of tree-grass associations to increasing interannual rainfall variability along the Kalahari rainfall gradient.

Chapters 5 and 6 describe greenshouse experiments to investigate the response of competitive relationships between CAM plants and other functional groups (i.e., $\mathrm{C}_{3}$ and $\mathrm{C}_{4}$ plants) to major global environmental change drivers (i.e., $\mathrm{CO}_{2}$ enrichment, drought, $\mathrm{N}$ deposition). Chapter 5 reports the results of growth chamber experiments under different $\mathrm{CO}_{2}$ and water conditions to investigate the impact on the competitive relationships between seedlings of Cylindropuntia imbricata (constitute CAM plants) and Bouteloua eriopoda $\left(\mathrm{C}_{4}\right.$ grass), which coexist in semiarid ecosystems across the Southwestern United States. Chapter 6 investigates how nutrient and water conditions affect competition between Mesembryanthemum crystallinum (a facultative CAM 
plant) and its interacting $\mathrm{C}_{3}$ species (Bromus mollis) found in California's coastal grasslands and the extent to which water stress, nutrients, and competition affect nocturnal carboxylation in $M$. crystallinum.

Chapters 7 and 8 develop mechanistic models to investigate the interactions involving woody plants, CAM plants, and grasses. In chapter 7, it is first shown that deep-rooted CAM plants in CAM-grass associations could perform hydraulic lift at a higher rate than trees in tree-grass associations in a relatively wet environment. Then it is shown that trees in tree-CAM associations may perform hydraulic descent at a higher rate than those in tree-grass associations in a dry environment and the high rate of hydraulic descent can even turn the facilitation of CAM plants by woody plants into competition. Chapter 8 describes the complex interactions involving three functional groups (i.e., woody plants, CAM plants, grasses) and shows that woody plants could directly facilitate CAM plants in access to soil water resources by shading; moreover they could indirectly facilitate CAM plants by suppressing grass transpiration through solar radiation reduction (SRR) and reducing the competitive effect of grasses on CAM plants in access to soil water resources. Chapter 9 summarizes the major conclusions of these studies and proposes potential future developments. 


\title{
2 An ecohydrological framework for grass displacement by woody plants in savannas
}

This chapter is adapted from Yu KL and D'Odorico P (2014), An ecohydrological framework for grass displacement by woody plants in savannas, Journal of Geophysical Research-

Biogeosciences, 119(3):192-206.

\begin{abstract}
During the past several decades woody plants have been encroaching into grasslands around the world. This transition in plant dominance is often explained as a state shift in bistable ecosystem dynamics induced by fire-vegetation feedbacks. These feedbacks occur when woody plants are able to displace grasses because of their better access to soil water and light. On the other hand, grasses can displace woody plants because of their ability to increase fire frequency and of the higher susceptibility of woody plants to fire-induced mortality. In this study, I present an ecohydrological framework to investigate the displacement of grasses by woody plants. Considering the effect of lateral root spread and of soil water and light limitations, I found that woody plant encroachment can substantially suppress grass production even without the presence of grazers. Bistable dynamics emerge as a result of the grass-fire feedback for a wide range of rainfall conditions, fire susceptibility, and woody plant growth rates.
\end{abstract}

\subsection{Introduction}

The encroachment of woody plants into grasslands has been widely documented in many regions of the world, including the southwestern United States (Archer et al., 1995; Van Auken, 2000), southern Africa (Moleele et al., 2002), Australia (Burrows et al., 1990), Asia (Binggeli, 1996), and the Mediterranean Basin (Maestre et al., 2009). This phenomenon typically entails either the transition to a savanna state with a mixed community of grasses and woody plants, or - more 
commonly - the replacement of the grasses with woody plants, often occurring in scattered patches bordered by bare soil (e.g., Schlesinger et al., 1990; Archer et al., 1995). This change in plant community composition affects the ecohydrological (Huxman et al., 2005) and geochemical (Hibbard et al., 2001) processes, as well as the provision of ecosystem services such as livestock grazing, sheltering of the soil surface, and carbon sequestration.

The transition from grasslands to woodlands has been associated with both exogenic and endogenic factors (D’Odorico et al., 2012). Exogenic factors are commonly invoked as drivers of the transition and include overgrazing, fire suppression (e.g., Scholes and Archer, 1997), increase in $\mathrm{CO}_{2}$ concentration (e.g., Morgan, 2007; Higgins and Scheiter, 2012), and long-term global changes in rainfall or temperature (e.g., Knapp et al., 2008). The endogenic factors typically involve positive feedbacks that may create bistable dynamics (D'Odorico et al., 2012). In other words, it has been argued that the positive feedbacks induce the emergence of two (alternative) stable states associated with grass and woody plant dominance, and that the encroachment of woody plants corresponds to a shift between these two attractors (Anderies et al., 2002; Okin et al., 2009a). This view is supported by the relatively abrupt and irreversible character of woody plant encroachment, as evidenced by the number of unsuccessful attempts to revert woodlands back to the grassland state (Havstad et al., 1999; Rango et al., 2005; Mata-Gonzalez, et al., 2007).

One of the major feedbacks arises from interactions between fires and grass biomass. It operates when woody plants have a competitive advantage over grasses in the access to the limiting resource (i.e., soil water) (Van Auken, 2000; Sankaran et al., 2004; D’Odorico et al., 2006a; Beckage et al., 2009). Therefore, in the absence of disturbances the system would tend to complete woody plant dominance. In this feedback, relatively dense grass canopy acting as a 
continuous stratum of fuel sustains intense fires, thereby killing woody plant seedlings. This positive feedback exists when (a) fire pressure decreases as grass biomass decreases (less grasses $\rightarrow$ less fires) (e.g., van Wilgen et al., 2000; Lehmann et al., 2011; Staver et al., 2011a b); (b) woody plant cover increases with decreasing fire pressure (less fires $\rightarrow$ more woody plants), as evidenced by fire control experiments (e.g., Bond et al., 2005; Runyan et al., 2012); (c) grass biomass decreases as woody plant cover increases (e.g., Oba et al., 2000; Eldridge et al., 2011 ). While the first two relations have been adequately documented, the ecohydrologic mechanisms underlying the decrease in grass cover resulting from woody plant encroachment still need to be clarified. There is a general consensus that overgrazing greatly reduces grass biomass and fire frequency, thereby favoring woody plant encroachment (e.g., Anderies et al., 2002; Okin et al., 2009a). However, it remains unclear whether woody plant encroachment itself can reduce grass cover even without the presence of grazers.

In arid and semiarid regions, soil water is the main limiting resource and is therefore considered the determinant of vegetation establishment and growth (Sankaran et al., 2005; Bond, 2008). Thus, to explain how woody plant cover affects grass production I need to investigate in detail the competitive relations between these two plant functional types with respect to soil water resources. Factors affecting root competition for soil moisture include climate conditions, soil characteristics, and the depth, lateral spread and degree of overlap of plant root systems (Casper and Jackson, 1997; Schenk and Jackson, 2005). The two-layer model proposed by Walter (1971) postulates that grasses are superior competitors for water in the upper soil layers, while woody plants have exclusive access to water stored in deeper soil layers. The generality of Walter's hypothesis, however, has been disputed (e.g., Smit and Rethman, 2000; Hipondoka et al., 2003; Sankaran et al., 2004; Beckage et al., 2009). In contrast to the two-layer model, other 
studies indicate that tree roots growing in coarse soils can spread laterally rather than vertically (e.g., Schenk and Jackson, 2002; Sternberg et al., 2004). Through these lateral roots trees can scavenge water from intercanopy areas (Casper et al., 2003; Sternberg et al., 2004; Barbier et al., 2008). Caylor et al (2005) proposed a model in which water uptake by the lateral roots can lead to the occurrence of wetter soils under tree canopies than in areas between canopies. However, the extent to which uptake by lateral tree roots can suppress grasses remains poorly quantified.

Another mechanism limiting grass production is associated with woody plant shading, which reduces solar radiation available to grass photosynthesis (Mordelet and Menaut, 1995; Lehmann et al., 2011). This mechanism explains the lower grass biomass found under tree canopies with respect to between canopy areas (e.g., Moustakas et al., 2013), especially in mesic savannas. In these environments the light limitation resulting from the presence of a relatively dense woody plant canopy limits grass production (e.g., Kim and Eltahir, 2004; Dohn et al., 2013). In this paper I account for the impact of grass suppression by soil water and light limitations on ecosystem dynamics.

I develop an ecohydrological framework to explain the mechanisms underlying grass displacement by woody plant encroachment. In particular, I evaluate the effect of lateral root spread and canopy shading on soil water and light limitations. By clarifying the relations existing between the fractional cover of woody plants and grass cover, this study sheds light on the processes underlying the grass-fire feedback, a mechanism that is crucial to the emergence of bistable grassland/woodland dynamics. 


\subsection{Methods}

\subsubsection{Modeling framework}

I develop an ecohydrological framework for the study of the dependence of grass cover on woody plant cover. I investigate the interaction of woody plants and grasses with soil water and light through a coupled energy and water balance model accounting for the competitive advantage of woody plants over grasses. The advantage is enhanced by lateral root spread and woody plant shading. In agreement with other studies (Van Auken, 2000; Sankaran et al., 2004; D’Odorico et al., 2006a; Beckage et al., 2009), I assume that woody plants have preferential access to soil water and that in the absence of disturbances (e.g., fires) they would outcompete grasses. This assumption is important in explaining how the rapid shift to a woodland state can be sustained by the grass-fire feedback (Van Auken, 2000; D’Odorico et al., 2006a). I assume that bottleneck effects associated with the higher susceptibility of woody plant seedlings and saplings to fires and drought affects vegetation dynamics only by slowing down the rate of woody plant establishment and growth (Bond, 2008). Moreover, I assume that fire frequency depends only on grass cover and that all woody plants have the same susceptibility to fire killings. The model couples the surface energy and soil water balance with vegetation dynamics for two plant functional types: woody plants and grasses. It accounts for the grass-fire feedback as a mechanism leading to the possible emergence of bistable dynamics. I parameterize the model using the savannas along the Kalahari Transect in Southern Africa as a case study.

In the model, I consider the surface energy and soil water balance for sites with and without woody plant canopies (hereafter called "under canopy" and "between canopy" areas, respectively). Five land cover components are considered: woody plant canopy, grasses under and between woody plant canopies, and bare soil under and between woody plant canopies. I 
denote the woody plant cover as $\mathrm{f}_{\mathrm{c}}(\%)$, and the grass density under and between canopies as $\mathrm{d}_{\mathrm{cg}}(\%)$ and $\mathrm{d}_{\mathrm{bg}}(\%)$, respectively. Accordingly, the bare soil density under and between canopies can be expressed as $d_{c s}=1-d_{c g}$ and $d_{b s}=1-d_{b g}$, respectively. The weighted fractions of area covered by grasses and bare soil are $f_{g}=\left(1-f_{c}\right) \times d_{b g}+f_{c} \times d_{c g}, f_{s}=$ $\left(1-f_{c}\right) \times d_{b s}+f_{c} \times d_{c s}$, respectively.

The average leaf area index $\left(\overline{\mathrm{LAI}}_{\mathrm{w}}, \mathrm{m}^{2} \mathrm{~m}^{-2}\right)$ of woody land patches is one of the crucial parameters in this model and is related to the average landscape-scale leaf area index through a representation of the landscape as a mosaic of canopy and between canopy areas resulting from a two-dimensional Poisson distribution of individual woody plants (Caylor et al., 2006). This representation accounts for vegetation canopy overlapping and provides a framework to calculate $\overline{\mathrm{LAI}}_{\mathrm{w}}$ as: $\overline{\mathrm{LAI}}_{\mathrm{w}}=\overline{\mathrm{n}}_{\mathrm{uc}} \times \mathrm{LAI}$, where $\overline{\mathrm{n}}_{\mathrm{uc}}$ is the average number of overlapping woody plant canopies in a woody patch and LAI the leaf area index of an individual woody plant canopy. Each woody plant individual is assumed to have only one canopy. The average number of woody plant canopies in the landscape can be expressed as $\overline{n_{c}}=f_{c} \overline{n_{u c}}+\left(1-f_{c}\right) \overline{n_{c b}}$, where $\overline{n_{c b}}$ is the number of woody plant canopies in between canopy areas. Because $\overline{\mathrm{n}_{\mathrm{cb}}}$ is by definition zero, I have $n_{c}=f_{c} \overline{n_{u c}}$. Following the approach by Caylor et al (2006), the average number of woody plant canopies in the landscape can be expressed as a function of woody plant $\operatorname{cover}\left(\mathrm{f}_{\mathrm{c}}\right): \overline{\mathrm{n}_{\mathrm{c}}}=$ $-\log \left(1-f_{c}\right)$. Thus, $-\log \left(1-f_{c}\right)=f_{c} \overline{n_{u c}}$. Rearranging these equations, the average leaf area index of woody patches can be calculated as:

$$
\overline{\mathrm{LAI}}_{\mathrm{w}}=-\frac{\log \left(1-\mathrm{f}_{\mathrm{c}}\right)}{\mathrm{f}_{\mathrm{c}}} \mathrm{LAI}
$$

I need to stress that a two-dimensional Poisson distribution assumes no clustering, while savanna trees are often observed in clumps. Because, to date, no close-form solutions exist for 
clustered two-dimensional stochastic processes of overlapping tree canopies, the Poisson process is here used as a simplified framework for a first-order approximation of the relationship between tree fractional cover and the average number of overlapping canopies in the landscape.

\subsubsection{Energy balance}

\subsubsection{Shortwave radiation}

The incident shortwave radiation is assumed to vertically irradiate the plant and soil surfaces (Caylor et al., 2005). Shortwave radiation exponentially decays through the plant canopy (Beer's law) (e.g., Campbell and Norman, 1998). Therefore, if $\mathrm{S}_{\text {sky }}$ is the average daytime incoming shortwave solar radiation $\left(\mathrm{W} \mathrm{m}^{-2}\right)$ above the canopy, the shortwave radiation upon the woody plant canopy (i.e., received by the canopy) is $S_{d(t, c)}=S_{\text {sky }}\left(1-\exp \left(-\mathrm{k}_{\mathrm{s}} \mathrm{LAI}_{\mathrm{w}}\right)\right)$, where $\mathrm{k}_{\mathrm{s}}$ is the extinction coefficient of shortwave radiation. Net radiation upon the woody plant canopy is $S_{n(t, c)}=\left(1-\alpha_{c}\right) S_{d(t, c)}$, where $\alpha_{c}$ is the shortwave albedo of the woody plant canopy. The incoming shortwave radiation under the woody plant canopy is $S_{d w n}=S_{s k y}-S_{d(t, c)}$. Net shortwave radiations for under-canopy grasses and bare soil are $S_{n(g, c)}=d_{c g}\left(S_{d w n}\left(1-\alpha_{g}\right)\right)$ and $\left(S_{n(s, c)}=\left(1-d_{c g}\right)\left[S_{d w n}\left(1-\alpha_{s}\right)\right]\right.$, respectively, where $\alpha_{g}$ and $\alpha_{s}$ are the shortwave albedo of grass canopy and bare soil, respectively. Net shortwave radiations for between-canopy grass and bare soil are $S_{n(g, b)}=d_{b g}\left(S_{\text {sky }}\left(1-\alpha_{g}\right)\right)$ and $S_{n(s, b)}=\left(1-d_{b g}\right)\left[S_{\text {sky }}\left(1-\alpha_{s}\right)\right]$, respectively.

\subsubsection{Longwave radiation}

Net longwave radiation $\left(L_{n}\right)$ is calculated as the difference between incoming and outgoing longwave radiation. Incoming longwave radiation is given by $\mathrm{L}_{\text {sky }}=\mathrm{e}_{\mathrm{a}} \sigma \mathrm{T}^{4}$, where $\mathrm{e}_{\mathrm{a}}, \sigma$ and 
$\mathrm{T}$ are the thermal emissivity, the Stefan-Boltzmann constant $(\sigma=5.670373 \times$ $10^{-8} \mathrm{~W} \mathrm{~m}^{-2} \mathrm{~K}^{-4}$ ), and the atmospheric temperature in degrees Kelvin at reference height (taken to be $10 \mathrm{~m}$ ), respectively. The thermal emissivity was expressed as $9.2 \times 10^{-6} \mathrm{~T}^{2}$ (Brutsaert, 1982). The net longwave radiation of vegetation is assumed to be zero during the daytime (Caylor et al., 2005). The outgoing longwave radiation of the soil under and between canopies is expressed as $\mathrm{L}_{\mathrm{o}(\mathrm{s}, \mathrm{c})}=\left(1-\mathrm{d}_{\mathrm{cg}}\right) \mathrm{e}_{\mathrm{s}} \sigma \mathrm{Te}_{(\mathrm{s}, \mathrm{c})}{ }^{4}$ and $\mathrm{L}_{\mathrm{o}(\mathrm{s}, \mathrm{b})}=\left(1-\mathrm{d}_{\mathrm{bg}}\right) \mathrm{e}_{\mathrm{s}} \sigma \mathrm{Te}_{(\mathrm{s}, \mathrm{b})}{ }^{4}$, respectively, where $\mathrm{e}_{\mathrm{s}}$ is the thermal emissivity of the soil, while $\mathrm{Te}_{(\mathrm{s}, \mathrm{c})}$, and $\mathrm{Te}_{(\mathrm{s}, \mathrm{b})}$ are the soil temperatures under and between canopies, respectively. Net longwave radiations for bare soil under and between canopies are then given by $\mathrm{L}_{\mathrm{n}(\mathrm{s}, \mathrm{c})}=\left(1-\mathrm{d}_{\mathrm{cg}}\right) \mathrm{L}_{\mathrm{sky}}-\mathrm{L}_{\mathrm{o}(\mathrm{s}, \mathrm{c})}$ and $\mathrm{L}_{\mathrm{n}(\mathrm{s}, \mathrm{b})}=$ $\left(1-d_{b g}\right) L_{s k y}-L_{o(s, b)}$, respectively.

\subsubsection{Net radiation and available radiation}

Net radiation $\left(R_{n}\right)$ for each landscape component is $R_{n}=L_{n}+S_{n}$. Estimations of soil evaporation and transpiration using the Priestley-Taylor approach depend on the quantity of energy available $(Q)$. For the two soil components, available energy is $Q_{s}=\left(1-C_{G}\right) R_{n}$, where $\mathrm{C}_{\mathrm{G}}$ is the ground flux coefficient, which expresses the fraction of $\mathrm{R}_{\mathrm{n}}$ contributing to ground heat flux. (Kustas and Norman, 1997; Lhomme and Monteny, 2000). The latent energy expenditure associated with plant interception ( $\mathrm{In}, \mathrm{mm} \mathrm{d}^{-1}$ ) is $\lambda_{\mathrm{v}} \rho_{\mathrm{w}} \mathrm{In}$, where $\rho_{\mathrm{w}}$ is the water density ( $\mathrm{kg} \mathrm{m}^{-}$ ${ }^{3}$ ) and $\lambda_{\mathrm{v}}$ the latent heat of vaporization determined as $\lambda_{\mathrm{v}}=3.1512 \times 10^{6}-2.38 \times$ $10^{3} \mathrm{Te}_{\mathrm{a}}\left(\mathrm{J} \mathrm{kg}^{-1}\right)$. 


\subsubsection{Water balance}

\subsubsection{Soil moisture dynamics}

Our study focuses on the effects of lateral root spread and assumes that grasses and woody plants have the same effective rooting depth $\left(\mathrm{Z}_{\mathrm{R}}, \mathrm{mm}\right)$. The soil moisture dynamics under and between canopies are expressed through soil water balance equations (Rodriguez-Iturbe et al.,1999; Laio et al., 2001):

$$
n Z_{\mathrm{R}} \frac{\mathrm{dS}_{\mathrm{c}}}{\mathrm{dt}}=\mathrm{I}_{\mathrm{c}}-\mathrm{E}_{\mathrm{c}}-\mathrm{L}_{\mathrm{c}}
$$

and

$$
n Z_{R} \frac{d S_{b}}{d t}=I_{b}-E_{b}-L_{b}
$$

where $\mathrm{n}$ is the soil porosity, $\mathrm{S}_{\mathrm{c}}$ and $\mathrm{S}_{\mathrm{b}}$ the relative soil moistures $\left(0<\mathrm{S}_{\mathrm{c}}, \mathrm{S}_{\mathrm{b}} \leq 1\right), \mathrm{I}_{\mathrm{c}}$ and $\mathrm{I}_{\mathrm{b}}$ the infiltration rates, $\mathrm{E}_{\mathrm{c}}$ and $\mathrm{E}_{\mathrm{b}}$ the sum of soil evaporation and transpiration, and $\mathrm{L}_{\mathrm{c}}$ and $\mathrm{L}_{\mathrm{b}}$ the drainage rates. The subscripts $\mathrm{c}$ and $\mathrm{b}$ refer to sites under and between canopies, respectively. The mean landscape soil moisture is then calculated as $S=f_{c} S_{c}+\left(1-f_{c}\right) S_{b}$.

\subsubsection{Precipitation}

Precipitation $(\mathrm{R}, \mathrm{mm})$ is modeled as a sequence of intermittent rainfall events using a marked Poisson process of storm occurrences with average rainfall frequency, $\lambda$, (events per day). Each storm is modeled as an exponentially distributed random depth with mean, $\mathrm{h},(\mathrm{mm}$ per event) (Rodriguez-Iturbe et al., 1999a; Laio et al., 2001). 


\subsubsection{Interception}

The maximum woody plant canopy interception $\left(\operatorname{In}_{\mathrm{c}, \max }\right)$ and the maximum grass interception $\left(\operatorname{In}_{\mathrm{g}, \max }\right)$ are both assumed to be constant $\left(\operatorname{In}_{\mathrm{c}, \max }=2 \mathrm{~mm}\right.$ and $\left.\operatorname{In}_{\mathrm{g}, \max }=1 \mathrm{~mm}\right)$ (Scholes and Walker, 1993). The minimum rainfall depths necessary to generate infiltration under and between canopies are $\operatorname{In}_{\mathrm{c} 0}=\operatorname{In}_{\mathrm{c}, \max }+\mathrm{d}_{\mathrm{cg}} \times \operatorname{In}_{\mathrm{g}, \max }$ and $\operatorname{In}_{\mathrm{b} 0}=\mathrm{d}_{\mathrm{bg}} \times \operatorname{In}_{\mathrm{g}, \text { max }}$, respectively. Therefore, the interception under and between canopies are given by $\operatorname{In}_{c}=\min \left(\operatorname{In}_{c 0}, R\right)$ and $\operatorname{In}_{\mathrm{b}}=\min \left(\operatorname{In}_{\mathrm{b} 0}, \mathrm{R}\right)$, respectively.

\subsubsection{Infiltration}

Rainfall in excess of $\operatorname{In}_{c}$ and $\operatorname{In}_{b}$ is available for infiltration. Infiltration is limited by the soil stotage capacity, which depends on soil depth, porosity and the current soil moisture. When rainfall is more than $\operatorname{In}_{c}$ and $\operatorname{In}_{\mathrm{b}}$, infiltrations under and between canopies are $\mathrm{Ic}=\min (\mathrm{R}-$ $\left.\operatorname{In}_{c}, \operatorname{nZr}\left(1-S_{c}\right)\right), I b=\min \left(R-\operatorname{In}_{b}, \operatorname{nZr}\left(1-S_{b}\right)\right)$, respectively (Laio et al., 2001).

\subsubsection{Transpiration and soil evaporation}

The rate of potential transpiration and soil evaporation are determined using the Priestley-Taylor equation. Using the Priestley-Taylor equation and excluding the effect of interception, the latent heat flux (LE) is expressed as: $L E=\frac{\alpha \Delta}{\Delta+\gamma} Q-\lambda_{v} \rho_{\mathrm{w}}$ In, where $\mathrm{Q}$ is the energy available for evapotranspiration $\left(Q=R_{n}\left(1-C_{G}\right)\right)$, In the interception rate, $\alpha=1.26$ the Priestley-Taylor coefficient, $\gamma$ the psychrometric constant $\left(\mathrm{Pa} \mathrm{K}^{-1}\right)$, and $\Delta$ the derivative of the relationship between saturation vapor pressure and air temperature $\left(\mathrm{Pa} \mathrm{K}^{-1}\right)(\mathrm{e} . \mathrm{g}$. , Campell and Norman, 1998). The soil heat fluxes of vegetated surfaces were assumed to be negligible (i.e., $\mathrm{C}_{\mathrm{G}} \approx 0$ ). 
The actual rates of transpiration or soil evaporation are then determined accounting for the dependence on soil moisture availability. To quantify the dependence of transpiration on soil moisture, I follow the approach developed by Rodriguez-Iturbe et al (1999) and represent the limitation of transpiration by soil moisture as:

$$
\mathrm{G}(\mathrm{S})=\left\{\begin{array}{lc}
0, & \mathrm{~S}<\mathrm{S}_{\mathrm{w}} \\
\frac{\mathrm{S}-\mathrm{S}_{\mathrm{w}}}{\mathrm{S}^{*}-\mathrm{S}_{\mathrm{w}}}, & \text { and } \mathrm{S}<\mathrm{S}^{*} \\
1, & \text { and } \mathrm{S} \geq \mathrm{S}^{*}
\end{array}\right.
$$

where $\mathrm{S}$ is the soil moisture, $\mathrm{S}^{*}$ the vegetation-specific value of relative soil moisture above which plants experience unstressed transpiration, and $S_{\mathrm{w}}$ the vegetation-specific wilting point at which point transpiration ceases. Because woody plants possess lateral roots that expand laterally into between canopy areas, when determining $\mathbf{G}(S)$, woody plants are assumed to experience the mean landscape soil moisture, $\mathrm{S}$, whereas grasses under and between canopies experience the local soil moisture, $S_{c}$ and $S_{b}$, respectively. The actual transpiration for each vegetation component is then determined according to: $T_{v e g}=\frac{L_{v} \mathrm{v}(S)}{\lambda_{v} \rho_{w}}$, where $L E_{v}$ is the latent heat flux for each vegetation component (i.e., woody plant canopy, grasses under canopy areas, grasses between canopy areas).

Actual soil evaporation also depends on soil moisture. The effect of soil moisture limitation on bare soil evaporation is represented by an exponential function (Boulet et al., 2000), $\beta(S)=$ $\exp (-\mathrm{k}(1-\mathrm{S}))$, where $\mathrm{k}$ is a (dimensionless) coefficient of limitation. The bare soil evaporation is then expressed as: $E_{\text {Soil }}=\frac{L E_{\text {soil }} \beta(S)}{\lambda_{\mathrm{v}} \rho_{\mathrm{w}}}$, where $L E_{\text {soil }}$ is the latent heat flux for each bare soil component (i.e., bare soil under canopy areas, bare soil between canopy areas). 
Woody plant canopy transpiration draws from both the under-canopy and between-canopy soil reservoirs because of lateral root spread, whereas the transpiration of grasses under and between canopies is localized to $S_{c}$ and $S_{b}$, respectively. The partitioning of the contributions to woody plant canopy transpiration into the under-canopy $\left(\mathrm{PAM}_{\mathrm{c}}\right)$ and between-canopy $\left(\mathrm{PAM}_{\mathrm{b}}\right)$ soil water stores are expressed as:

$\mathrm{PAM}_{\mathrm{c}}=\left\{\begin{array}{cc}\frac{\mathrm{S}_{\mathrm{c}}-\mathrm{S}_{\mathrm{wt}}}{\left(\mathrm{S}_{\mathrm{c}}+\mathrm{S}_{\mathrm{b}}\right)-2 \mathrm{~S}_{\mathrm{wt}},} & \mathrm{S}_{\mathrm{c}}>\mathrm{S}_{\mathrm{wt}} \\ 0, & \text { and }_{\mathrm{c}} \leq \mathrm{S}_{\mathrm{wt}}\end{array}\right.$

and

$$
\operatorname{PAM}_{\mathrm{b}}=\left\{\begin{array}{cc}
\frac{\mathrm{S}_{\mathrm{b}}-\mathrm{S}_{\mathrm{wt}}}{\left(\mathrm{S}_{\mathrm{c}}+\mathrm{S}_{\mathrm{b}}\right)-2 \mathrm{~S}_{\mathrm{wt}},} & \mathrm{S}_{\mathrm{b}}>\mathrm{S}_{\mathrm{wt}} \\
0, & \text { and }_{\mathrm{b}} \leq \mathrm{S}_{\mathrm{wt}}
\end{array}\right.
$$

where $S_{w t}$ is the wilting point of woody plants. To quantify the effects of woody plant cover $\left(\mathrm{f}_{\mathrm{c}}\right)$ on between canopy soil moisture, the portion of woody plant transpiration drawing from the between-canopy soil reservoir is expressed as:

$$
T_{s b}=\operatorname{PAM}_{b} T_{(t, c)} \frac{f_{c}}{1-f_{c}},
$$

where $\mathrm{T}_{(\mathrm{t}, \mathrm{c})}$ is the woody plant canopy transpiration.

The total transpiration and soil evaporation for the canopy portion of the landscape is $\mathrm{ET}_{\mathrm{c}}=$ $\mathrm{T}_{(\mathrm{g}, \mathrm{c})}+\mathrm{E}_{(\mathrm{s}, \mathrm{c})}+\mathrm{PAM}_{\mathrm{c}} \mathrm{T}_{(\mathrm{t}, \mathrm{c})}$, where $\mathrm{T}_{(\mathrm{g}, \mathrm{c})}$ and $\mathrm{E}_{(\mathrm{s}, \mathrm{c})}$ are the grass transpiration and soil evaporation under canopy areas, respectively. The total transpiration and soil evaporation for between canopy areas is expressed as:

$$
\mathrm{ET}_{\mathrm{b}}=\mathrm{T}_{(\mathrm{g}, \mathrm{b})}+\mathrm{E}_{(\mathrm{s}, \mathrm{b})}+\mathrm{PAM}_{\mathrm{b}} \mathrm{T}_{(\mathrm{t}, \mathrm{c})} \frac{\mathrm{f}_{\mathrm{c}}}{1-\mathrm{f}_{\mathrm{c}}},
$$


where $\mathrm{T}_{(\mathrm{g}, \mathrm{b})}$ and $\mathrm{E}_{(\mathrm{s}, \mathrm{b})}$ are the grass transpiration and soil evaporation between canopy areas, respectively. The total transpiration and soil evaporation for the whole landscape scale is then $E T=f_{c} E T_{c}+\left(1-f_{c}\right) E T_{b}$.

\subsubsection{Drainage}

It is assumed that there is no interaction with underlying soil layers, and consequently excess soil moisture is expected to drain to field capacity (Caylor et al., 2005). When soil moisture is larger than field capacity $\left(\mathrm{S}_{\mathrm{fc}}\right)$, the drainage under and between canopies are $\mathrm{L}_{\mathrm{c}}=\mathrm{nZ}_{\mathrm{R}}\left(\mathrm{S}_{\mathrm{c}}-\mathrm{S}_{\mathrm{fc}}\right)$ and $\mathrm{L}_{\mathrm{b}}=\mathrm{nZ}_{\mathrm{R}}\left(\mathrm{S}_{\mathrm{b}}-\mathrm{S}_{\mathrm{fc}}\right)$, respectively.

\subsubsection{Vegetation dynamics and equilibrium states}

Changes in woody plant cover are modeled as a logistic growth with a mortality term accounting for the effects of fire:

$$
\frac{d f_{c}}{d t}=b f_{c}\left(1-\frac{f_{c}}{f_{c m a x}}\right)-d f_{c} g\left(f_{g}\right)
$$

where $\mathrm{b}$ is the woody plant growth coefficient, $\mathrm{f}_{\mathrm{cmax}}$ the woody plant carrying capacity (i.e., the maximum canopy cover allowed by the limiting resources), $d$ a parameter determining the death rate of woody plants by fires, and $g\left(f_{g}\right)$ a term expressing the control of grass fuel on fire frequency. The relationship between $\mathrm{f}_{\mathrm{cmax}}$ and mean annual rainfall (MAP) is shown in Supplementary Figure 2.1 (based on Sankaran et al (2005)). The ratio d/b greatly affects the bistable dynamics of woody plants-grass-fire interactions. I assume that $d$ is constant and $b$ increses with the amount of rainfall during the growing seaon, consitent with other studies (e.g., Dauber et al., 2005; Toledo et al., 2011). For simplicty, the woody plant growth rate is expressed as a linear function of the growing season rainfall, $\mathrm{R}(\mathrm{mm})$ : $\mathrm{b}=\mathrm{c} \times \mathrm{R}$, where $\mathrm{c}\left(\mathrm{mm}^{-1}\right)$ is a 
constant. To investigate the senstivity of the dynamics to changes in the ratio $\mathrm{d} / \mathrm{c}$,two different values of $d / c$ were considered (Supplementary Figure 2.2). Fire frequency is a function of grass biomass. Using data by van Wilgen et al. (2000), I express $g\left(f_{g}\right)$ as:

$$
g\left(f_{g}\right)=\frac{\eta f_{g}{ }^{2}}{1+(\eta-1) f_{g}{ }^{2}}
$$

where $\eta$ is a coefficient estimated as $\eta=5$ to match observations (Figure 2.1) at Kruger National Park (South Africa) by van Wilgen et al. (2000).

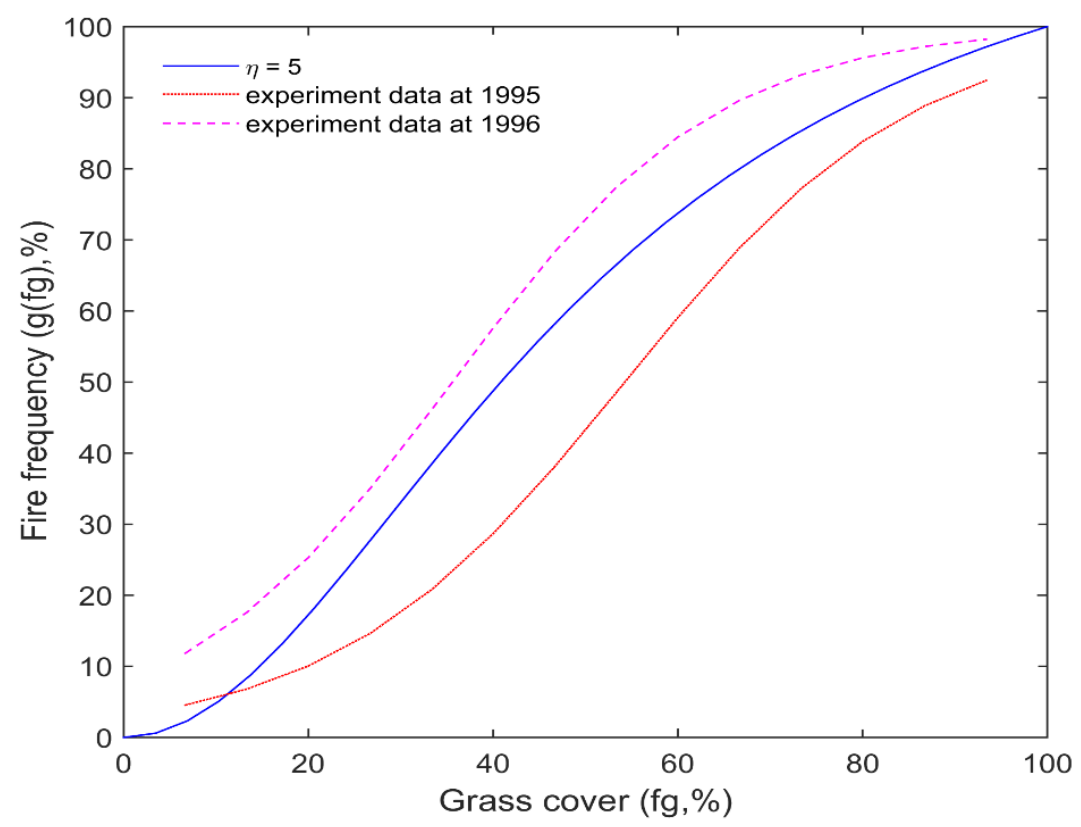

Figure 2-1: Dependence between fire frequency and fuel load (i.e., grass cover) at Kruger National Park, South Africa (experiment data from van Wilgen et al., 2000).

Dynamics of grass cover are represented as a logistic growth:

$$
\frac{d f_{g}}{d t}=\beta f_{g}\left(1-\frac{f_{g}}{f_{g \max }\left(f_{c}\right)}\right)
$$


where $\beta$ and $f_{\text {gmax }}\left(f_{c}\right)$ are the grass growth coefficient and the grass carrying capacity, respectively. $f_{\text {gmax }}\left(f_{c}\right)$ depends on the woody plant cover.

To quantify the dependence of $f_{\text {gmax }}$ on $f_{c}$, I firstly consider the effect of soil water limitation and then the impact of light limitation. For water limitation, I determine the maximum grass density under $\left(\mathrm{d}_{\text {cgmw }}\right)$ and between $\left(\mathrm{d}_{\text {bgmw }}\right)$ canopies corresponding to different values of $f_{c}$. In other words, because of the initial assumption that canopy cover $f_{c}$ is independent of grass density (in the absence of fire), I first assign values of $f_{c}$ and then calculate the maximum grass density that the soil water balance could sustain. To this end, I simulate the soil moisture dynamics for different values of $d_{c g}$ or $d_{b g}$ (depending on whether it is a canopy or between canopy site) and calculate the average soil moisture. Conditions associated with an average soil moisture during the growing season greater than a critical value - here taken equal to 1.7 times the soil moisture at wilting point of grasses - are considered to be suitable for grass growth. The highest $d_{c g}\left(\right.$ or $d_{b g}$ ) corresponding to conditions suitable for grass survival is then the value of $d_{\text {cgmw }}$ or $\left(d_{\text {bgmw }}\right)$ corresponding to that value of $f_{c}$. To investigate the sensitivity to changes in the critical soil moisture required to sustain maximum grass density, two different values of the wilting point for grasses were considered. To account for light limitation, I determined the maximum density of grasses under and between canopies as $\mathrm{d}_{\mathrm{cgm}}=\mathrm{ld}_{\mathrm{cgmw}}$ and $\mathrm{d}_{\mathrm{bgm}}=$ $l d_{\text {bgmw }}$, respectively, where $l$ is the light limitation coefficient determined as the ratio of actual net photosynthesis rate to saturated net photosynthesis rate (Supplementary Figure 2.3), based on leaf-level physiological measurements (O'Halloran, 2007). $\mathrm{f}_{\text {gmax }}\left(\mathrm{f}_{\mathrm{c}}\right)$ is then given by:

$$
f_{\text {gmax }}\left(f_{c}\right)=\left(1-f_{c}\right) d_{\text {bgm }}+f_{c} \times d_{\text {cgm }} \text {, }
$$

2.2.4.1 Stable states of the system 
The equilibrium states of the ecosystem are obtained by taking the temporal derivatives equal to zero on the left-hand side of equations (1) and (3). Setting the temporal derivative of equation (1) equal to zero and combining equation (1) with equation (2), I obtain:

$$
\mathrm{f}_{\mathrm{c}}=\mathrm{f}_{\mathrm{cmax}}\left(1-\frac{\mathrm{d \eta} \mathrm{f}_{\mathrm{g}}^{2}}{\mathrm{~b}\left(1+(\eta-1) \mathrm{f}_{\mathrm{g}}^{2}\right.}\right)
$$

Likewise, setting the temporal derivative of equation (2) equal to zero and combining equation (2) with equation (4), I obtain:

$$
f_{g}=\left(1-f_{c}\right) d_{b g m}+f_{c} \times d_{c g m}
$$

Equilibrium states of the system satisfy both equations (5) and (6), and can therefore be determined as intersections of the corresponding curves in the $\left(f_{c}, f_{g}\right)$ domain.

\subsubsection{Parameterization of the model}

The model is parameterized for the case of savannas along the Kalahari Transect in Southern Africa. This region has a relatively homogenous soil along a south-to-north rainfall gradient, and provides an excellent experimental setting to investigate changes in plant community composition associated with different rainfall regimes (Koch et al., 1995). It has been argued that in the Kalahari environment variations in temperature are much less important than rainfall in determining vegetation composition and structure (Porporato et al., 2003). I represent rainfall variability using a Poisson process (e.g., Laio et al., 2001) and use parameters (e.g., rainfall parameters, average daytime incoming solar radiation $\left(\mathrm{S}_{\text {sky }}\right)$, mean atmospheric temperature $\left(\mathrm{Te}_{\mathrm{a}}\right.$ ), and elevation (he)) from the sites of Tshane and Mongu as representative of semiarid and mesic conditions (Supplementary Table 2.1) existing along the Kalahari Transect (Scanlon and Albertson, 2004a). Sensitivity test indicates that model results are insensitive to variations in 
atmospheric temperature and site elevation. The model operates under the assumption of a statistically homogeneous growing season climate (Laio et al., 2001; Porporato et al., 2003). The rainy season coincides with the growing season and is assumed to last 210 days from October to May, which accounts for $90 \%$ of mean annual rainfall (MAR). The mean of the exponential distribution of the rainstorm depth is taken to be a constant $\left(\mathrm{h}=11 \mathrm{~mm} \mathrm{~d}^{-1}\right)$, consistent with the finding of a relatively uniform distribution of this parameter across the Kalahari rainfall gradient (Porporato et al., 2003). In this study, variations in precipitation along the Kalahari rainfall gradient are determined only by changes in the average rainfall frequency (i.e., frequency of rainy days), $\lambda$. The average daytime incoming solar radiation $\left(\mathrm{S}_{\mathrm{sky}}\right)$ is determined based on energy flux measurements (30 minute averages) during the SAFARI 2000 growing season campaign (Scanlon and Albertson, 2004b). Soil temperature under and between canopies is determined as a function of $\mathrm{S}_{\text {sky }}$ and air temperature based on two empirical relationships developed by Caylor et al (2005): $\mathrm{Te}_{(\mathrm{s}, \mathrm{b})}=\exp \left(\mathrm{b}_{1}+\mathrm{b}_{2} \mathrm{~S}_{\mathrm{sky}}+\mathrm{b}_{3} \mathrm{Te}_{\mathrm{a}}\right)$ and $\mathrm{Te}_{(\mathrm{s}, \mathrm{c})}=\mathrm{a}_{1}+$ $\mathrm{a}_{2}$ Sdwn $+\mathrm{a}_{3} \mathrm{Te}_{\mathrm{a}}$, respectively, where $\mathrm{a}_{1,2,3}$ and $\mathrm{b}_{1,2,3}$ are empirical coefficients, and $\mathrm{S}_{\text {sky }}$ is expressed as $\mathrm{W} \mathrm{m}^{-2}$. The soil water balance equations are integrated numerically with a time step of 0.1 day. The leaf area index of an individual woody plant canopy (LAI) is taken to be 2.5 in my study to match observations from the Kalahari Transect in Southern Africa (e.g., Caylor et al., 2005). Values of other model parameters are shown in Supplementary Table 2.2.

\subsection{Results and discussion}

Increases in woody plant cover significantly reduced grass cover in both wet (average rainfall frequency, $\lambda=0.4 \mathrm{~d}^{-1}$ and average rainstorm depth, $\left.\mathrm{h}=11 \mathrm{~mm}\right)$ and dry $(\lambda$ $=0.2 \mathrm{~d}^{-1}$ and $\mathrm{h}=11 \mathrm{~mm}$ ) environments (Figure 2.2). 


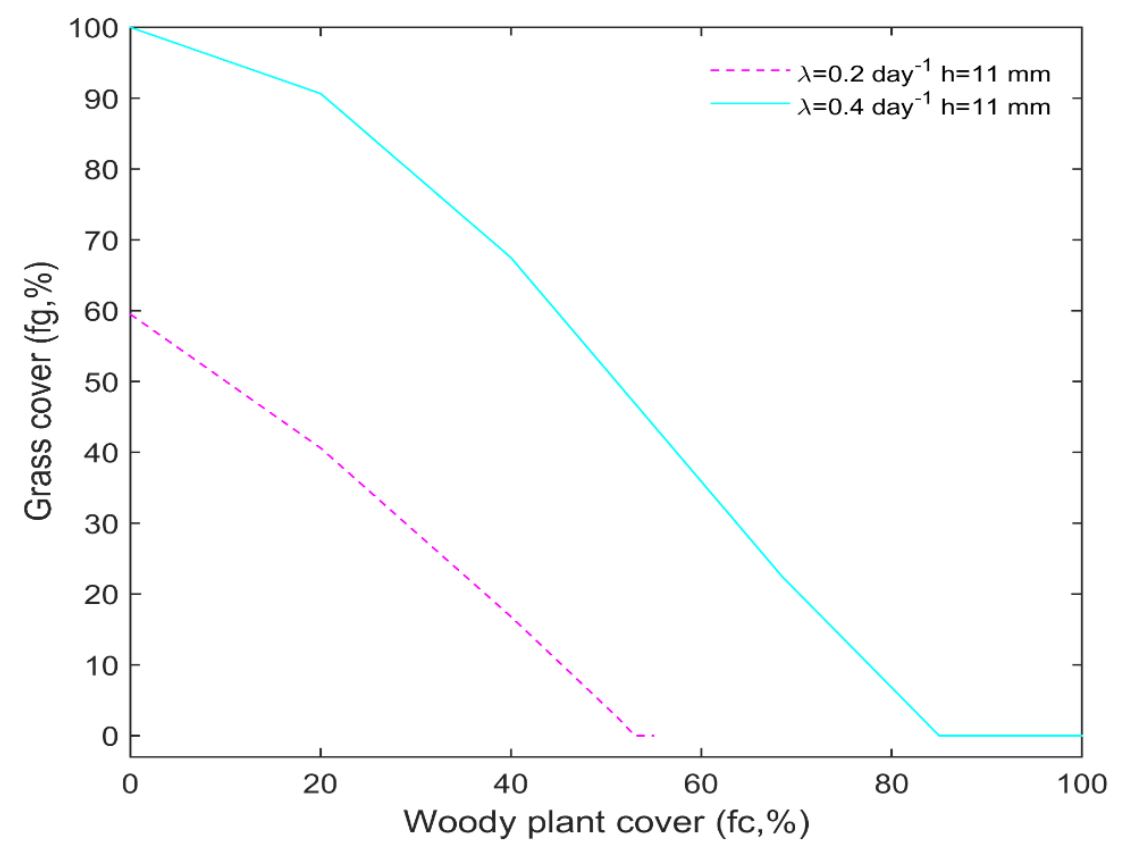

Figure 2-2: The relationship between woody plant cover $\left(f_{c}\right)$ and grass cover $\left(f_{g}\right)$. Solid and dashed lines represent wet (average rainfall frequency, $\lambda=0.4 \mathrm{~d}^{-1}$ and average rainstorm depth, $\mathrm{h}$ $=11 \mathrm{~mm})$ and $\operatorname{dry}\left(\lambda=0.2 \mathrm{~d}^{-1}\right.$ and $\left.\mathrm{h}=11 \mathrm{~mm}\right)$ environment, respectively.

These results are consistent with experimetal observations. For example, in a global synthesis of the impacts of woody plant encroachment on ecosystem structure and functioning, Eldridge et al (2011) found a decrease in grass cover in landscapes affected by woody plant encroachment. The ability of woody plant encroachment to reduce the grass cover is a crucial component of the firevegetation feedback commonly invoked to explain the emergence of the grassland/woodland bistable dynamics. Past research argued that overgrazing decreases grass cover, thereby reducing fire frequency and thus favoring woody plant encroachment (e.g., Anderies et al., 2002; Okin et al., 2009a). My study shows that the reduction in grass cover is likely an effect of woody plant encroachment. The ecohydrological mechanisms underlying this causal relationship are based on the competitive interactions between woody plants and grasses with respect to soil water 
resources and light. Empirical studies point to the ability of woody plants to exploit soil water resources both under and between canopy areas (due to lateral root spread) (e.g., Casper et al., 2003; Caylor et al., 2005; Barbier et al., 2008) and therefore suppress grass production between canopies. Additionally, under canopy areas light limitation on grass photosynthesis due to woody plant shading further reduces the grass cover, especially in wet environments (where the woody plant canopy is denser) (e.g., Mordelet and Menaut, 1995; Lehmann et al., 2011; Dohn et al., 2013; Moustakas et al., 2013). I found that in arid environments grass cover is limited mainly by water availability with no major shading effects because the tree cover is relatively low. As tree cover $\left(\mathrm{f}_{\mathrm{c}}\right)$ increases (i.e., in wetter environments), grass growth is predominately limited by light except for the intercanopy patches, which cover an overall small portion of the landscape. Overall the results shown in Figure 2.2 indicate that woody plant encroachment can markedly reduce grass cover, thereby allowing for the establishment of a positive feedback among grass cover, fire dynamics and woody plant encroachment (D’Odorico et al., 2012).

Bistable dynamics are characterized by three intersections - between the curves represented by equations (5) and (6) - corresponding to two stable states separated by an unstable one. If the system is in one of the two stable equilibrium points, it will remain in that state unless it is perturbed, while, if it is in an intermediate condition it will converge to one of the two stable states, depending on the initial condition. The grass-fire feedback can lead to the emergence of bistable dynamics both at the dry $\left(\lambda=0.2 \mathrm{~d}^{-1}\right.$ and $\left.\mathrm{h}=11 \mathrm{~mm}\right)$ and wet $\left(\lambda=0.4 \mathrm{~d}^{-1}\right.$ and $\left.\mathrm{h}=11 \mathrm{~mm}\right)$ sites (Figure 2.3). If the initial state of the system is a grassland, the system is unable to spontaneously shift to a woodland state because the grassland is a stable configuration (Figure 2.3). 

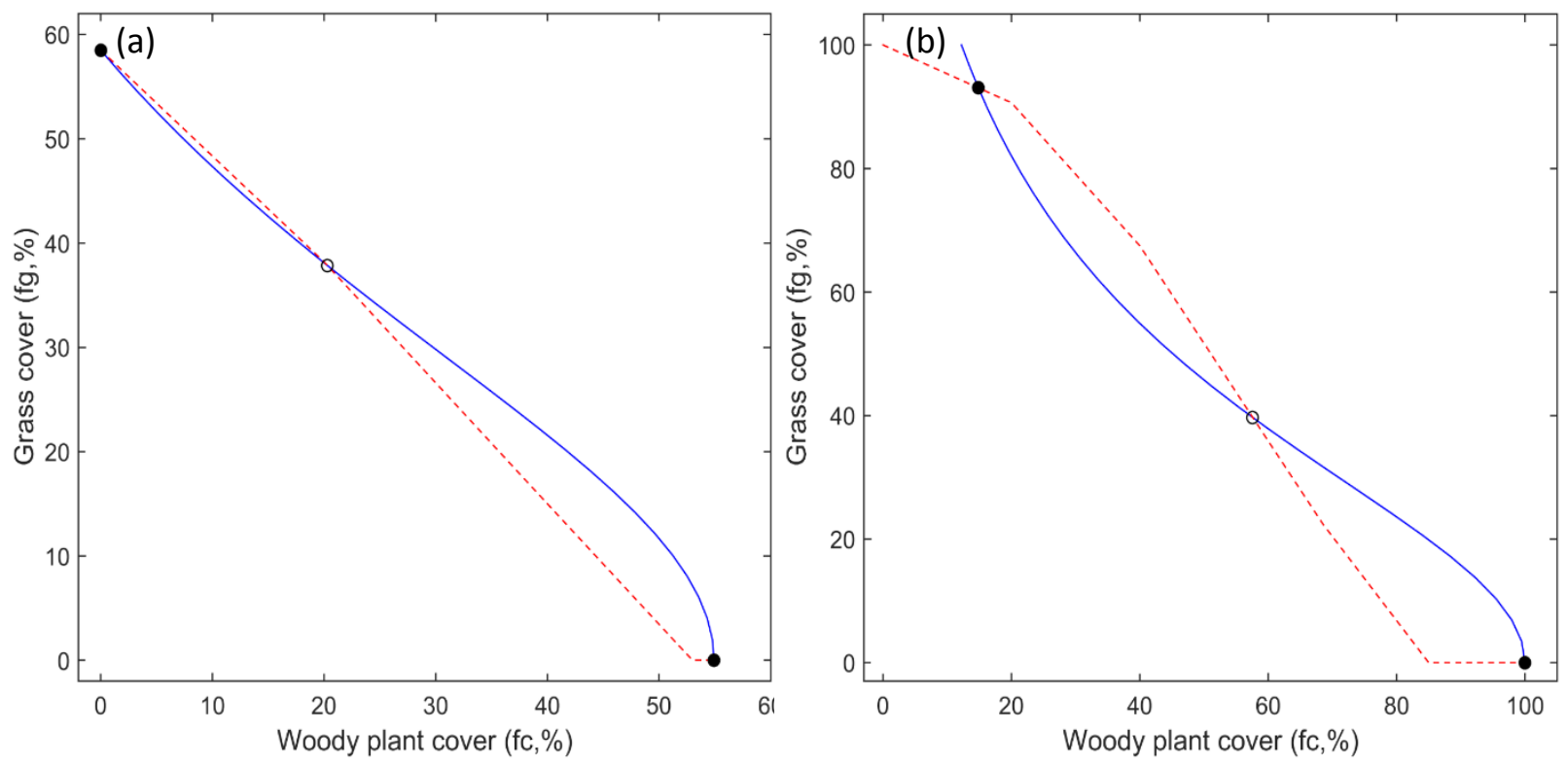

Figure 2-3: Stable (solid black intersection points) and unstable (white intersection point) states of the dynamics obtained as intersection of curves given by equations (5) (solid line) and (6) (dashed line) in two different rainfall regimes: (a) dry (average rain frequency, $\lambda=0.2 \mathrm{~d}^{-1}$ and average rainstorm depth, $h=11 \mathrm{~mm})$ environment and (b) wet $\left(\lambda=0.4 \mathrm{~d}^{-1}\right.$ and $\left.\mathrm{h}=11 \mathrm{~mm}\right)$ environment.

But if a disturbance pushes the system away from the attraction domain of the grassland (i.e., beyond the unstable state in Figure 2.3), a self-sustained sequence of processes (i.e., grass cover reduction, decrease in fire pressure, and encroachment of woody plants) sustains the shift to the woodland state. Such a shift can be highly irreversible because the state with woody plant dominance is also stable and the system will remain locked therein even after the removal of the disturbance. As noted in the introduction, woody plant encroachment is often considered as a state shift in bistable ecosystem dynamics because of the abrupt and apparently irreversible character of this transition, which suggests the existence of internal thresholds and a "fold-type bifurcation" (Figure 2.3) (D’Odorico et al., 2012). 
Precipitation is an important variable that can affect the bistable dynamics of vegetation (Figure 2.4a). When the mean annual rainfall (MAR) is less than $430 \mathrm{~mm}\left(\mathrm{~S}_{\mathrm{wg}}=0.06\right)$, the ecosystem has only one stable state of grassland. In these conditions, woody plants are either not capable of surviving because of water limitations, or, because of the relatively high grass biomass: fires are strong enough to kill all the woody plants and prevent the establishment of a tree or shrub canopy. This result is consistent with the observations of grassland dominance in the southern part of the Kalahari transect (e.g., Scholes et al., 2002). Similar results were obtained by Staver and Levin (2012), though their model allowed for the existence of some woody plants even in dry environments because of the weakening of the fire regime with low grass covers.

Our results show that bistable dynamics associated with grasslands and woodlands occur with the mean annual rainfall (MAR) in the $450-1050 \mathrm{~mm}$ range (with $\mathrm{S}_{\mathrm{wg}}=0.06$ ) (Figure $2.4 \mathrm{a}$ ). Because of killings by fires sustained by significant grass cover, grasslands remain a stable state of the system; at the same time, because of their ability to suppress grasses, thereby reducing the fire pressure, woodlands are also a stable state of the system. When MAR is greater than 1050 mm, forest (woody plant cover $=100 \%$ ) and savanna (woody plant cover $>5 \%$ ) occur as alternative stable states. The existence of savanna as a stable state is due to the decrease in $d / b$ (the ratio between the fire-induced mortality and the growth rate of woody plants) with increasing values of MAR (the rate of post-fire woody plant recovery increases in more humid environments). The emergence of these bistable dynamics is consistent with a number of other studies and experimental observations reported in Table 2.1. 
Table 2-1 A summary of evidence of the occurrence of the fire-vegetation feedback (FVF) and bistability

(Bi).

\begin{tabular}{|c|c|c|c|c|c|}
\hline Region & $\begin{array}{l}\text { MAR } \\
(\mathrm{mm})\end{array}$ & Findings & FVF & $\mathrm{Bi}$ & Reference \\
\hline Serengeti (East Africa) & 1000 & $\begin{array}{l}\text { Elephants reduce woody plant cover and } \\
\text { fires maintain the stable state of grasslands }\end{array}$ & Yes & Yes & $\begin{array}{l}\text { Dublin et al } \\
(1990)^{1}\end{array}$ \\
\hline $\begin{array}{l}\text { Brazilian Amazon } \\
\text { (South America) }\end{array}$ & & $\begin{array}{l}\text { Fire has the potential to transform the tropical forest into } \\
\text { savannas }\end{array}$ & Yes & & $\begin{array}{l}\text { Cochrane } \\
\text { et al (1999) }\end{array}$ \\
\hline Catalonia (Spain) & 550 & $\begin{array}{l}\text { Large tussock grass invasion replaces shrublands because of } \\
\text { a fire-grass feedback }\end{array}$ & Yes & & $\begin{array}{l}\text { Vilà et al (2001) and } \\
\text { Grigulis et al (2005) }\end{array}$ \\
\hline \multirow{3}{*}{ USA and East Africa } & & $\begin{array}{l}\text { Clearing of tropical savannas results in warmer and drier } \\
\text { climate, accelerated fire frequencies, and further tree cover } \\
\text { loss }\end{array}$ & Yes & & Hoffmann et al (2002) \\
\hline & $\begin{array}{l}500- \\
900\end{array}$ & $\begin{array}{l}\text { Fires drive and maintain } \mathrm{C} 4 \text { grasslands expansion into } \\
\text { woodlands during the late Miocene }\end{array}$ & Yes & & $\begin{array}{l}\text { Keeley and } \\
\text { Rundel (2005) }\end{array}$ \\
\hline & & Trees act as engineers that modify fire regimes & Yes & Yes & Beckage et al (2009) ${ }^{1}$ \\
\hline $\begin{array}{l}\text { Wet Tropics of Far } \\
\text { North Queensland } \\
\text { (Australia) }\end{array}$ & $\begin{array}{l}1000- \\
2000\end{array}$ & $\begin{array}{l}\text { Distinctive features of the vegetation supporting alternative } \\
\text { stable states }\end{array}$ & Yes & Yes & $\begin{array}{l}\text { Warman and Moles } \\
(2009)^{1}\end{array}$ \\
\hline $\begin{array}{l}\text { Everglades National } \\
\text { Park (USA) }\end{array}$ & 1500 & $\begin{array}{l}\text { The invasive shrubs suppress fire disturbance and convert a } \\
\text { savanna to an invasive-dominated forest }\end{array}$ & Yes & Yes & $\begin{array}{l}\text { Stevens and Beckage } \\
(2009)^{1}\end{array}$ \\
\hline $\begin{array}{l}\text { Africa, Australia, } \\
\text { and South America }\end{array}$ & & $\begin{array}{l}\text { Evidence of forests, savannas, and grasslands as alternative } \\
\text { stable states }\end{array}$ & Yes & Yes & Hirota et al $(2011)^{1}$ \\
\hline $\begin{array}{l}\text { Africa, Australia, and } \\
\text { South America }\end{array}$ & $\begin{array}{l}1000- \\
2500\end{array}$ & Savanna and forest exist as alternative stable states & Yes & Yes & $\begin{array}{l}\text { Staver et al } \\
(2011 \mathrm{a} \mathrm{b})^{1}\end{array}$ \\
\hline $\begin{array}{l}\text { Kruger National Park } \\
\text { (South Africa) }\end{array}$ & $\begin{array}{l}600- \\
700\end{array}$ & $\begin{array}{l}\text { In the absence of fires the ecosystem becomes a woodland. } \\
\text { Fire frequency increases with grass biomass. }\end{array}$ & Yes & & $\begin{array}{l}\text { van Wilgen et al. }(2000)^{1} \\
\text { Runyan et al }(2012)^{1}\end{array}$ \\
\hline Africa & & $\begin{array}{l}\text { Increase in } \mathrm{CO}_{2} \text { concentrations induces an abrupt vegetation } \\
\text { shift to woodlands }\end{array}$ & Yes & Yes & $\begin{array}{l}\text { Higgins and Scheiter } \\
\text { (2012) }\end{array}$ \\
\hline $\begin{array}{l}\text { Emas National Park } \\
\text { (Brazil) }\end{array}$ & $\begin{array}{l}1200- \\
2000\end{array}$ & $\begin{array}{l}\text { Fire-vegetation feedbacks drive and maintain } \\
\text { forests and savannas as two stable states }\end{array}$ & Yes & Yes & $\begin{array}{l}\text { Dantas et al } \\
(2013)^{1}\end{array}$ \\
\hline
\end{tabular}

Note: ${ }^{1}$ indicates the direct evidence which supports my results of bistability. 
The presence of this bistable regime leads to strong nonlinearities and hysteresis in the ecosystem dynamics with important implications on the ecosystem's response to "external" drivers such as intense browsing, overgrazing, or increase in $\mathrm{CO}_{2}$ concentration (e.g., Anderies et al., 2002; D’Odorico et al., 2006a; Higgins and Scheiter, 2012). For example, Higgins and Scheiter (2012) indicated that in Africa an abrupt vegetation shift to woodlands could result from the increase in atmospheric $\mathrm{CO}_{2}$ concentrations. In contrast, intense browsing reduces woody plant cover thereby favoring the grasslands (e.g., Anderies et al., 2002; D’Odorico et al., 2006a).

Interestingly, the unstable intersection corresponds to higher values of woody plant cover and tends to become closer to the stable woodland state in wetter environments (Figure 2.4a, b). This occurs because grass production is less limited by water availability despite the presence of woody plant roots spreading laterally into areas between canopy areas. It is important to stress, however, that - consistent with the experimental findings by Wang et al. (2009) - I have not considered limitations of grass production that in wet environments could result from insufficient availability of nutrients such as nitrogen, phosphorus and potassium (e.g., Hooper and Johnson, 1999; Van Duren and Pegtel, 2000; Ludwig et al., 2001). Moreover, I have not considered a possible increase in woodland resilience that could result from the reduction in fire frequency in the more mesic enviroments (e.g., Cochrane, 2003).

In the presence of fire-vegetation feedbacks, the interplay between woody plant growth and fires determines the bistable ecosystem dynamics (e.g., Hirota et al., 2011; Lehmann et al., 2011; Staver et al., 2011a b). Therefore, any factor affecting the rates of woody plant canopy growth and the intensity of fire could affect the bistable regime. As shown in Figure 2.4a, an increase in the wilting point of grasses extends the bistable range to values of MAR as low as $385 \mathrm{~mm}$, 
while increasing the resilence of the woodland state. Likewise, a reduction in the $d / b$ ratio enhances the resilience of the woodland state (Figure 2.4b).
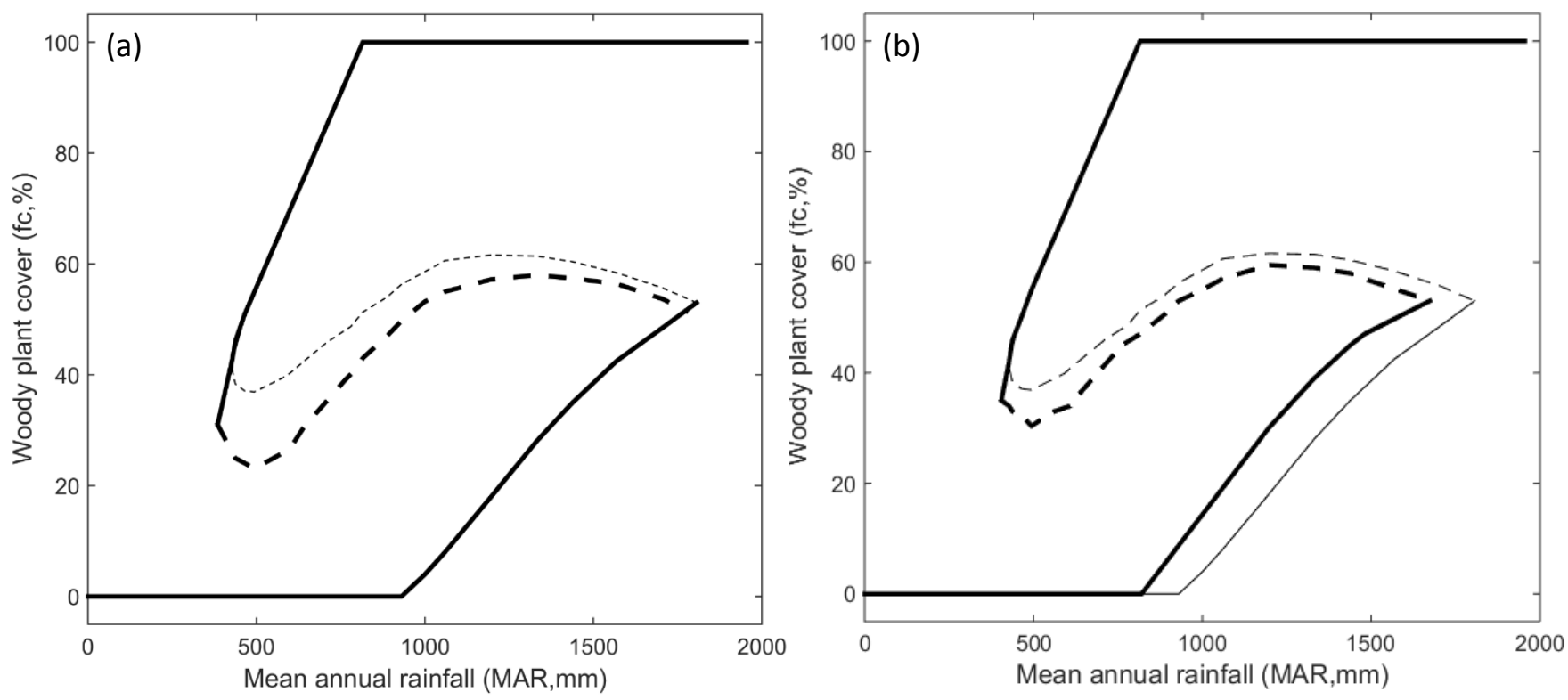

Figure 2-4: Stable (solid line) and unstable (dashed line) states as a function of mean annual rainfall (MAP). (a) wilting point for grass, $S_{\mathrm{wg}}=0.065$ (thick lines) and $\mathrm{S}_{\mathrm{wg}}=0.06$ (fine lines);

(b) with different ratios between fire-induced mortality and growth: $\mathrm{d} / \mathrm{b}=750 \mathrm{~mm}$ (thick lines) and $\mathrm{d} / \mathrm{b}=840 \mathrm{~mm}$ (fine lines).

While the parameter b (woody plant growth rates) is expected to depend on species interactions, climate, soil charateristics and disturbances not related to fires (e.g., Holdo, 2006; Coomes and Allen, 2007), woody plant susceptibility to fire (i.e., d) varies with species and growth stage (Bond, 2008).

In this model I assume that woody plants are better competitors for soil water resources and therefore can outcompete grasses in the absence of disturbances. I need to stress, however, that the competitive dominance of woody plants with respect to grasses changes both with life- 
history stage as well as across environmental gradients (Sankaran et al., 2004). In fact, in some ecosystems grasses are better competitors where they share the same belowground rooting space with woody seedlings and saplings (e.g., Scholes and Archer, 1997; House et al., 2003; Bond, 2008). Likewise, I do not account for the fact that saplings are more susceptible to fires than adult trees (e.g., Bond, 2008). In the absence of major bottleneck effects, however, these assumptions are not expected to greatly affect the overall dynamics of grass-woody plant interaction. The higher sensitivity of tree saplings to grass competition and fires is mainly expected to slow-down woody plant growth and enhance woody plant susceptibility to fires.

In summary, grass-fire feedbacks are often considered the cause of bistable vegetation dynamics explaining the irreversible transition from a grassland to a woodland state. Crucial to these dynamics is the ability of woody plants to displace grasses in the absence of fires, the ability of fires to decrease woody plant cover, and the ability of grasses to increase fire frequency. This study has elucidated the ecohydrological mechanisms underlying grass displacements by woody plants. It is shown that the displacement is associated with soil water and light limitations which may lead to substantial reductions in grass production following woody plant encroachment. It has also shown that when the ability of woody plants to displace grasses is accounted for, the resulting fire-vegetation feedback induces bistable dynamics for a wide range of rainfall conditions, fire susceptibility, and woody plant growth rates. In these conditions, grasslands become susceptible to (almost) irreversible shifts to the alternative woodland stable state under the action of disturbances. 


\section{Potential of grass invasions in fireproof desert shrublands to create novel ecosystem states under variable climate}

This chapter is adapted from Yu KL and D'Odorico P, From facilitative to competitive interactions between woody plants and plants with Crassulacean Acid Metabolism (CAM): the role of hydraulic descent, Ecohydrology (In press).

\section{Abstract}

The invasion of exotic grasses into shrublands is a major disturbance to dryland ecosystems. The presence of exotic grasses enhances the occurrence of wildfire in landscapes that had not evolved in the presence of fire, leading to high rates of mortality of the native vegetation. Exotic grasses could be more prone to water stress and mortality than the shrubs they replaced and may not establish during drought, facts that are crucial in ecosystems undergoing increased climatic variability. Here I develop a process-based modeling framework to investigate the complex dynamics resulting from the introduction of exotic grasses under variable climate. I find that the system converges towards different steady states, depending on the magnitude of climatic variability. While in the absence of climate fluctuations the shrubland state is replaced by an exotic grassland, interannual climate variability may inhibit grass invasion and stabilize the shrubland state. However, climatic variability also gives rise to a novel third, unvegetated state, with grass invasion being followed by drought, grass mortality, and intense soil erosion. Most of the research on climate change effects on ecosystems has historically concentrated on the ecological impact of shifts in mean climate conditions. This study shows that changes in the variance are also important when shifts in vegetation composition (e.g., species invasions) result in different susceptibility to climatic variability. In the presence of random climate fluctuations ecosystems can display steady states that differ from those that would exist under a constant climate or with a climate trend. 


\subsection{Introduction}

Biological invasions are recognized as major contributors to global environmental change (Vitousek et al., 1997; Mooney and Cleland, 2001). It has been observed that biological invasions affect ecosystem dynamics not only through their direct impact on resource competition and pool of available species (e.g., Olsson et al., 2012), but also indirectly through their ability to modify the disturbance regime. This disturbance-mediated effect of species invasions on ecosystems is observed when the invader is functionally different from the native species, i.e., when it exhibits some traits that (i) affect the disturbance regime and (ii) are missing in the native population (D'Antonio, 2000). For example, the invasion of desert shrublands by exotic annual and perennial grasses has been observed to lead to an increase in fire frequency and intensity due to the increase in grass fuel and in connectivity of vegetation cover (Okin et al., 2009b). The introduction of fires in shrubland ecosystems, where burning has not been historically a major selective force, results in an increase in the mortality rates of shrubland species, particularly if they are not adapted to fire (e.g., Bond et al., 2005; Runyan et al., 2012). The loss of shrub biomass further enhances the establishment and spread of invasive grasses (D'Antonio, 2000). Known as "the fire cycle" (D'Antonio and Vitousek, 1992), this positive feedback leads to the replacement of fire-intolerant native shrubs with exotic grasses (Figure 3.1). The grass-fire feedback may induce stable grassdominated vegetated states in arid and semiarid environments (Grigulis et al., 2005; Keeley and Rundel, 2005), even when shrubs have competitive advantage with respect to grasses in the access to resources (Okin et al., 2009a).

The case of exotic grass species invasions into fireproof desert shrublands is the mirror image of shrub encroachment into former desert grasslands, which has been occurring in drylands worldwide over the past two centuries (Van Auken, 2000; Ravi et al., 2009, D'Odorico et al., 2012). 
Invasion by exotic - both annual and perennial - grasses coupled with changes to the fire regime has been observed more recently and has been documented for several dryland regions around the world, including Western North America, Australia, and Southern Africa (D'Antonio, 2000). For example, native shrublands in the Sonoran Desert have extremely low fire frequency, due to a sparse canopy and the absence of a continuous fuel (grass) layer. The low fire frequency led to the evolution of plants in the region that are not fire-adapted. Thus in this region, fire sustained by the exotic grasses can kill native vegetation thereby threatening the continued existence of native fireintolerant desert shrubland (McDonald and McPherson, 2011).

The typical changes in dryland vegetation associated with the invasion of desert shrublands by exotic grasses are shown in Figure 3.1. I look at this process as a sequence of three major stages plus a potentially novel fourth state characterized by different plant community composition, fire frequency, and ecosystem processes. The initial state (State I) of the system is a fireproof landscape with fire-intolerant native shrubs. After the introduction of invasive grasses, the continuity of the grass layer contributes to fire spread across the landscape. At this stage invasive grasses and shrubs coexist (State II). The transition from States I to II involves the colonization and establishment of exotic grasses as in most cases of biological invasions (Theoharides and Dukes, 2007). This process has been often associated with anthropogenic introduction (Dukes and Mooney, 1999) and/or increase in atmospheric $\mathrm{CO}_{2}$ concentrations and changes in climate (i.e., precipitation and/or temperature) (Smith et al., 2000; Davis et al., 2000; Ziska et al., 2005; Sorte et al., 2013). As noted earlier, this mixed grass-shrub community is not stable because its persistence is prevented by fire dynamics. In State II shrub vegetation is prone to fire-induced mortality due to increase in fire pressure resulting from the introduction of flammable grasses. Thus, the fire cycle, i.e., the positive feedback between fires and vegetation, accelerates the rate of grass invasion at the 
expenses of the native vegetation (Grigulis et al., 2005; Keeley and Rundel, 2005; Miller et al., 2010). Thus, the system shifts to a state dominated by exotic grasses with no native shrub plants (State III). At this stage, flammable grasses cover the landscape (McDonald and McPherson, 2011).

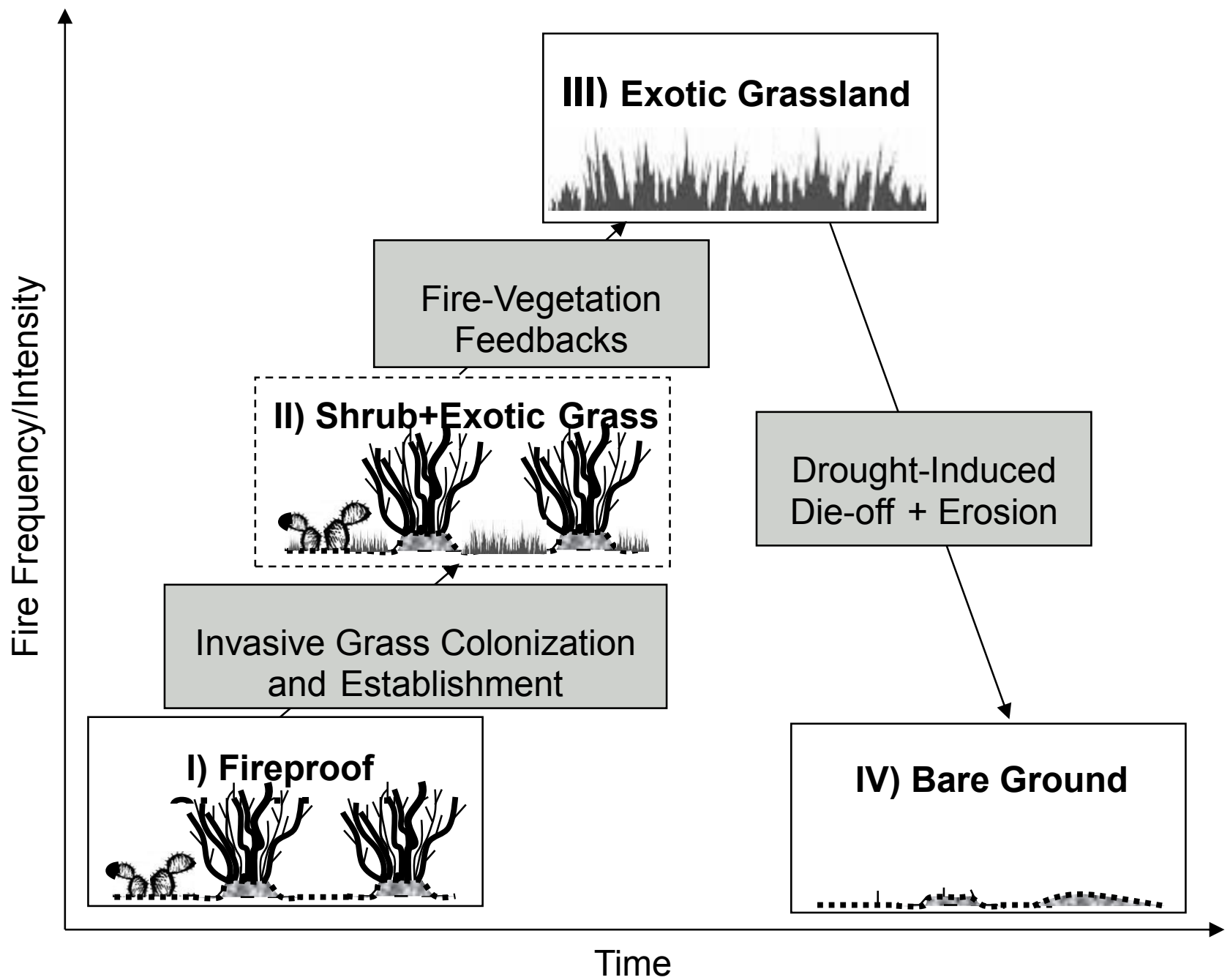

Figure 3-1: A conceptual representation of the possible four stages of vegetation transition in desert scrublands as affected by grass invasions.

Climate in arid and semiarid regions is notoriously variable, and is expected to become increasingly so, even if there is no long-term aridification trend worldwide; it has been argued that in the arid regions of western North America, a drying trend will likely occur in the next 50 
years (Seager et al., 2007, IPCC, 2013). Moreover, the increase in aridity is often associated with an increase in interannual climate variability (Nicholson 1980; Easterling et al., 2000; IPCC, 2013). A considerable number of studies have already addressed the transitions from States I to II and from States II to III (e.g., D'Antonio and Vitousek, 1992; D'Antonio, 2000), while it is less clear whether grass invasions could interact with fire dynamics in the presence of increased rainfall variability to convert these once fire-proof shrubland landscapes into highly degraded barren landscapes (State IV).

In this study, I investigate the idea that increase in interannual rainfall variability increases the frequency of extended period of low precipitation (i.e., drought years) and thus invasive grass cover (annuals or perennials) might be greatly reduced due to plant mortality under drought years to produce a novel unfertile state with little or no vegetation cover, especially when the grasses exhibit a special drought-susceptibility or lag in regrowth after drought (State IV) (e.g., Peake at al., 1979; Tilman and Haddi, 1992; Franklin et al., 2006). Although it is possible that the system could transition from states I to IV without going through III, in this study I focus on the transitions of the vegetation states presented in Figure 3.1 to investigate whether grass invasions and climate change could act in concert to induce land degradation. I suggest that this novel degraded state may be stable if the landscape is prone to wind and/or water erosion, which deplete soil resources and the seed bank, thereby potentially inhibiting the re-establishment of vegetation, including shrubs, even during wet years (Parsons et al., 2003; Okin et al., 2006; Okin et al., 2009a; Okin et al., 2009b; Ravi et al., 2009; Alvarez et al., 2012). The emergence of this novel degraded state as a result of grass invasions and interannual rainfall fluctuations would require a long term drought (i.e., on decadal time scales) following the transition from state I to state III. 
Studies on ecosystem dynamics in response to interannual rainfall variability are crucial but largely restricted by the short record of available data and technical capability to measure all relevant variables (e.g., Fatichi and Ivanov, 2014; Ng et al., 2015). To overcome these limitations, I develop a minimalist process-based model of coupled soil resource-vegetation dynamics to investigate the interactions between native shrubs and exotic invasive grasses. By clarifying the role of increased climate fluctuations in determining land degradation induced by grass invasions, this study contributes to a better understanding of ecosystem susceptibility to biological invasions and climate change.

\subsection{Methods}

\subsubsection{Modeling framework}

The effect of biotic-abiotic interactions on ecosystem dynamics has been often investigated with simple deterministic models accounting both for interspecific competition and for feedbacks with environmental conditions and disturbance regime. Some of these models have been developed to study changes in plant community composition in a variety of dryland ecosystems, and to show the emergence of alternative stable states in their deterministic dynamics (e.g., Noy-Meir, 1975; May, 1977; Walker et al., 1981; Anderies et al., 2002; Van Langevelde et al., 2003; Okin et al., 2009a). Here I use a similar approach to investigate a different process: the effect of grass invasions on the temporal dynamics of desert shrublands in a randomly fluctuating environment. To this end, I develop a stochastic process-based model of vegetation-resource dynamics accounting for the interactions between shrubs and grasses, and for their coupling with the dynamics of soil resources. This model assumes that (i) shrubs and grasses compete for the same soil resources (e.g., Smit and Rethman, 2000; Hipondoka et al., 2003; Beckage et al., 2009),

although I acknowledge that in some dryland ecosystems shrubs could have deeper roots than 
grasses (i.e., Walter's two layer hypothesis) (Walter, 1971; Eagleson and Segarra, 1985). (ii) even though in some ecosystems grasses might be stronger competitors than shrubs (especially at the seedling and sapling stages) (e.g., Scholes and Archer, 1997), here I consider the case of ecosystems where - in the absence of fires and climate fluctuations - shrubs have preferential access to soil resources and therefore are in competitive advantage with respect to exotic grasses (Van Auken, 2000; Sankaran et al., 2004; Beckage et al., 2009; Yu and D’Odorico, 2014a).; (iii) fires act as a source of disturbance for the native shrub population, i.e., the rate of fire-induced shrub mortality is proportional to the grass biomass (i.e., to the fuel load) (van Wilgen et al., 2000; Beckage et al., 2009); (iv) the carrying capacities for native shrubs $\left(S_{m a x}\right)$ and exotic grasses $\left(G_{\max }\right)$ depend on the available soil resources, $R$, mainly soil nutrients as well as on soil moisture, which varies from year to year as a result of interannual climate fluctuations. Thus the carrying capacities of shrubs and grasses are here accounted for by treating $S_{\max }$ and $G_{\max }$ as random variables with mean dependent on $R$. The sensitivity to these fluctuations is stronger in the invasive grasses than in the native shrubs, which are better adapted to droughts (e.g., Tilman and Haddi, 1992). Thus, the same climate fluctuations cause stronger variability in the carrying capacity of grasses than in that of shrubs (see the section on stochastic dynamics for details).

\subsubsection{Soil resource dynamics}

The state variables, native shrub biomass $(S)$, invasive grass biomass $(G)$, and soil resources $(R)$ have the dimensions of mass per unit area $\left(\mathrm{M} \mathrm{L}^{-2}\right)$. The temporal variability of $R$ is the result of an imbalance between the rates of soil resource accumulation, $R_{\mathrm{A}}$, and loss, $R_{\mathrm{E}}$

$$
\frac{d R}{d t}=R_{A}-R_{E}
$$


Overall, the accumulation of soil resources is favored by the presence of plant canopies because of their ability to reduce erosion and favor deposition (e.g., Okin et al., 2009a). In fact, vegetation tends to provide a more favorable depositional environment for sediments transported by wind and water (Schlesinger et al., 1990). Thus, the accumulation rate, $R_{\mathrm{A}}$ (with the dimensions $\left(\mathrm{M} \mathrm{L}^{-2} \mathrm{~T}^{-1}\right)$ ) is an increasing function of the total plant biomass, $G+S$ (Figure 3.1).

$R_{A}=c_{1}\left(1-e^{-c_{2}(S+G)}\right)$

with $c_{1}\left(\mathrm{M} \mathrm{L} \mathrm{L}^{-2} \mathrm{~T}^{-1}\right)$ and $c_{2}\left(\mathrm{~L}^{2} \mathrm{M}^{-1}\right)$ being two parameters determining the magnitude of the accumulation rate and its sensitivity to $S+G$, respectively. The rate, $R_{\mathrm{E}}\left(\mathrm{M} \mathrm{L}^{-2} \mathrm{~T}^{-1}\right)$, of resource loss associated with soil erosion is proportional to the amount of existing resources, $R$, and decreases with increasing vegetation biomass

$R_{E}=\left(c_{3}+c_{4} e^{-c_{5}(S+G)}\right)$

where the first of the two terms between brackets expresses the biomass independent erosion rate, while the second term accounts for the dependence of $R_{\mathrm{E}}$ on total plant biomass. The constants $c_{3}$ and $c_{4}$ have dimensions of $\left(\mathrm{M} \mathrm{L}^{-2} \mathrm{~T}^{-1}\right)$ and $c_{5}$ has dimensions of $\left(\mathrm{L}^{2} \mathrm{M}^{-1}\right)$.

\subsubsection{Vegetation dynamics}

Following other studies (e.g., Andeires et al., 2002; Van Langevelde et al., 2003; Beckage et al., 2009; Yu and D'Odorico, 2014a), the rate of change of shrub biomass is proportional to the existing shrub biomass, $S$, and to the resources available for new shrub growth, $S_{\max }-S$, while fireinduced disturbance kills shrubs at a rate that is proportional to the existing shrub biomass and to fire frequency, $f(G)$,

$\frac{d S}{d t}=\alpha \mathrm{S}\left(S_{\max }-S\right)-\beta f(G) S$ 
with $\alpha$ and $\beta\left(\mathrm{L}^{2} \mathrm{M}^{-1} \mathrm{~T}^{-1}\right)$ being two parameters determining the rates of shrub growth and of fireinduced mortality, respectively. As assumed above, in the absence of fires and climate fluctuations shrubs are in competitive advantage with respect to exotic grasses in the access to soil resources (Van Auken, 2000; Beckage et al., 2009; Yu and D’Odorico, 2014a); thus, the resources available to shrubs are expressed as $S_{\max }$ while the resources available to grasses are expressed as $G_{\max }-S$. Experimental evidence suggests that fire frequency is an increasing function of grass biomass (van Wilgen et al., 2000). Even though in this study I do not model fire as a sequence of intermittent events occurring at a given frequency (D’Odorico et al., 2006a), I account for the effect of grasses on fires by expressing the fire pressure as a deterministic function of grass biomass. Following van Wilgen et al (2000), I express fire frequency, $f(G)$, as:

$$
f(G)=\frac{\exp (q)}{1+\exp (q)}
$$

where $q=-2.47+2.35 G$.

Consistent with other studies (e.g., Andeires et al., 2002; Van Langevelde et al., 2003; Beckage et al., 2009; Yu and D’Odorico, 2014a), this model does not account for resprouting of shrubs after fires, a trait that is species-specific and is expected to favor State II (e.g., Chidumayo, 2004; Vest et al., 2004; Moreira et al., 2012). Thus, grasses may limit shrub growth only through fire dynamics but not through a preferential access to the available resources.

Similarly, the rate of change of grass biomass is modeled as proportional to grass biomass and to the resources left available to new grass growth (i.e., to $G_{\max }-(S+G)$ ), while a grass invasion term accounts for the effects of grass invasion, 
$\frac{d G}{d t}=\gamma \mathrm{G}\left(G_{\max }-S-G+F_{a} S\right)+I \delta\left(t-t_{I}\right)$

with $\gamma\left(\mathrm{M} \mathrm{L}^{-2} \mathrm{~T}^{-1}\right)$ being a parameter determining the rates of grass growth, $F_{\mathrm{a}} S$ a facilitation term expressing the facilitation effects of shrubs on grasses through shade ( $F_{\mathrm{a}}$, a facilitation coefficient $)$ (e.g., Holzapfel and Mahall, 1999; Yu and D’Odorico, $2015 \mathrm{a}$ b), and I (M L $\left.{ }^{-2} \mathrm{~T}^{-1}\right)$ being a onetime event of invasive species introduction occurring at time $t=t_{I}$. Thus, if $F_{\mathrm{a}}$ is positive, shrubs have a facilitative effect on grasses while if $F_{\mathrm{a}}$ is negative, shrubs only exert a competitive effect on grasses. During this event a relatively small amount of exotic grass biomass is successfully introduced and established. The function $\delta\left(t-t_{I}\right)$ in equation (5) is equal to 1 at time $t=t_{I}$ and zero, otherwise. Note that invasion of grasses into bare soil state during periods of sufficient water availability may lead to a small increase in soil resources and multiple invasions may convert the bare soil state to grassland state if no drought conditions are experienced before significant establishment. To account for the faster dispersal and growth of invasive grasses with respect to the native shrubs, the constant of proportionality in equation (5), $\gamma$, must be greater than $\alpha$.

\subsubsection{Stochastic dynamics}

If the carrying capacities are constant and have the same value for shrubs and grasses, the vegetation dynamics expressed by (4) and (5) have only one stable state, which is either $\left(G=G_{\max }\right.$, $S=0)$ or $\left(G=0, S=S_{\max }\right)$, depending on the relative importance of the parameters controlling shrub growth and fire-induced mortality. To investigate how these dynamics are modified by interannual climate variability that influences water availability and by feedbacks between vegetation and available resources, $R$, I express both $G_{\max }$ and $S_{\max }$ as random variables with synchronous fluctuations with mean, $\left\langle G_{\max }\right\rangle=\left\langle S_{\max }\right\rangle=R$, and lognormal distribution (Okin et al., 2009a). To account for the higher sensitivity of invasive grasses to drought occurrences, I assume that the 
same climate fluctuations induce a stronger variability in the response of invasive grasses than in native shrubs by using two different values for the standard deviation of the lognormal distribution of the carrying capacities for grasses $\left(\sigma_{\mathrm{G}}\right)$ and shrubs $\left(\sigma_{\mathrm{S}}=k \sigma_{\mathrm{G}}\right)$, with $\sigma_{\mathrm{G}}>\sigma_{\mathrm{S}}$ (i.e., $\left.k<1\right)$. More specifically, the simulation of both $G_{\max }$ and $S_{\max }$ is conducted by sampling at each time step a random number from a normal distribution of random numbers with mean 0 and standard deviation 1 (i.e., $\mathrm{N}(1,0)$ ). The same number is then converted into a value, $G_{\max }$, with lognormal distribution, mean $R$, and standard deviation $\sigma_{\mathrm{G}}$, and a value $S_{\max }$ with the same mean (i.e, $\left\langle G_{\max }\right\rangle=\left\langle S_{\max }\right\rangle$ $=R$ ) and standard deviation $\sigma_{\mathrm{S}}=k \sigma_{\mathrm{G}}$. In this way the carrying capacities of grasses and shrubs are random variables with the same mean $R$ and synchronous fluctuations of different amplitude (i.e., different standard deviation).

I use the model to investigate the interplay among vegetation dynamics, climate fluctuations and changes in available resources. To this end, I consider the case of a system in which, in the absence of fluctuations (i.e., $\sigma_{\mathrm{G}}=\sigma_{\mathrm{S}}=0$ ), exotic grasses are able to successfully establish and to completely displace the native shrubs (i.e., in this case the stable state of the deterministic system is $G=G_{\max }, S=0$ ). I then investigate the stochastic dynamics (i.e., $\sigma_{\mathrm{G}}>0, \sigma_{\mathrm{S}}>0$ ). For different values

of $\sigma_{\mathrm{G}}$ and $\sigma_{\mathrm{s}} \mathrm{I}$ run 1,000 iterations of the process and calculate the probability that the system reaches a steady state with shrub biomass ("shrub state"), grass biomass ("grass state"), or bare soil ("crash state") in which shrub and grass biomass are zero and soil resources fall to zero. Each simulation is run for 3,000 time steps using as initial conditions the native shrub state (i.e., $G=0$; $S=R=1$ ), and allowing for one-time invasive species introduction at time $t_{l}=250$.

\subsection{Results}

In the model developed in this study, I characterize the state of the system using three state variables: native shrub biomass $(S)$, invasive grass biomass $(G)$, and soil resources $(R)$. The 
dynamics of $S$ and $G$ are limited both by the resources, $R$, which varies in time as a result of random interannual rainfall fluctuations that affect $G_{\max }$ and $S_{\max }$. These fluctuations impose random variability on the carrying capacities of $S$ and $G$, with standard deviations, $\sigma_{\mathrm{S}}$ and $\sigma_{\mathrm{G}}$, respectively. Due to the higher drought sensitivity of grasses, $\sigma_{\mathrm{G}}$ is bigger than $\sigma_{\mathrm{S}}$. I look at the asymptotic state of the system (i.e., at the end of the simulation period), and classify it as "shrub state", "grass state" or "crash state", depending on whether it exhibits nonzero shrub biomass (while $G=0$ ), nonzero grass biomass (while $S=0$ ), or zero grass and shrub biomass, respectively. All simulations resulted in one of these three final states after 3000 iterations.

The results of this analysis for different levels of climate fluctuations (i.e., different values of $\sigma_{\mathrm{G}}$ and $\left.\sigma_{\mathrm{S}}=\mathrm{k} \sigma_{\mathrm{G}}\right)$ show that, in the deterministic case $\left(\sigma_{\mathrm{G}}=\sigma_{\mathrm{S}}=0\right)$, the fire cycle leads to the successful dispersal of invasive grasses (Figure 3.2). In this case, the system reaches a stable state dominated by exotic grasses and no other mechanism further disturbs this state. As the amplitude of climate fluctuations is increased, the probability for the system to reach a stable grassland state decreases, while the probability of converging to a stable shrubland state increases along with the probability of "crashing" to a resource depleted bare soil state. Thus, climate fluctuations can completely reverse the behavior of the system and allow for the stable existence of the shrub state, while in the deterministic counterpart of this process, the stable state of the system is a grassland. For intermediate amplitude of climate fluctuations the probability of reaching the grassland or the shrubland states can be comparable, while there is an even higher probability of crashing to the unvegetated state (Figure 3.2). 


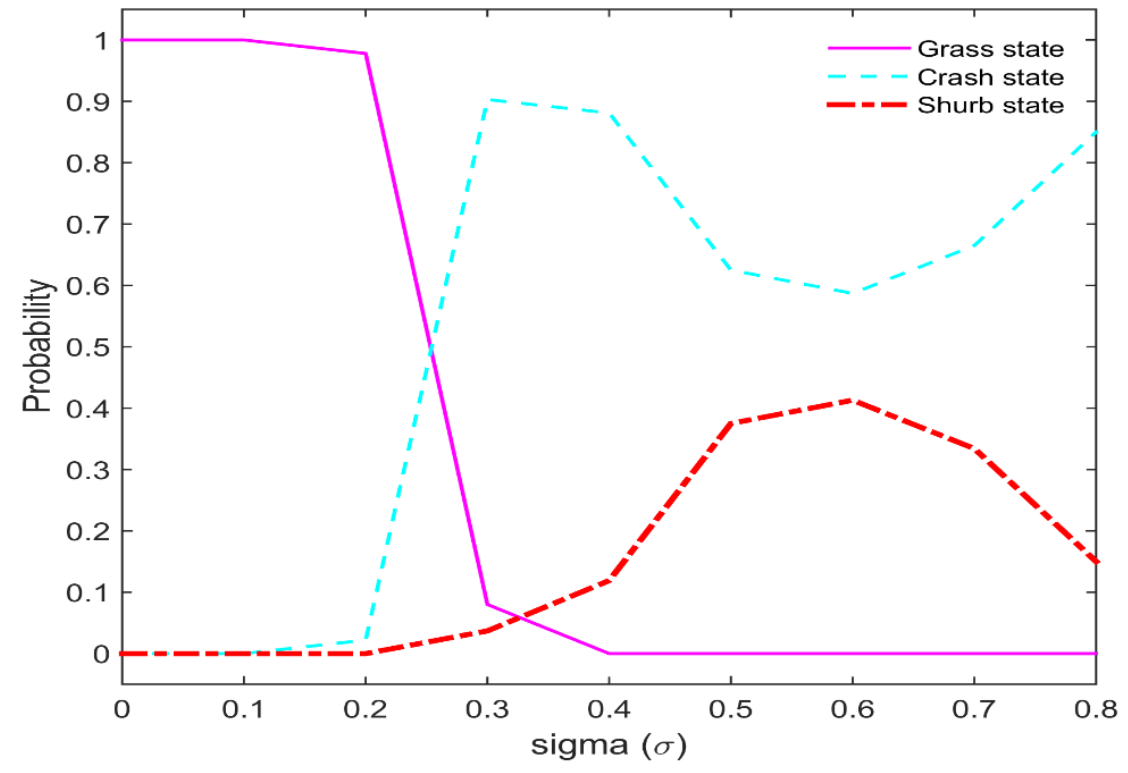

Figure 3-2: Probability of being in one of three possible states at the final state $(\mathrm{t}=3000)$ as a function of $\mathrm{G}$ (shown for the case of one time grass invasion with $\alpha=0.5 ; \beta=1.3 ; \gamma=0.85$; $\mathrm{c} 1=0.08 ; \mathrm{c} 2=2 ; \mathrm{c} 3=0.06 ; \mathrm{c} 4=0.06 ; \mathrm{c} 5=10 ; \mathrm{Fa}=0 ; \sigma \mathrm{S}=0.7 \sigma \mathrm{G})$.

The modeling results also show that in the deterministic case $\left(\sigma_{\mathrm{G}}=\sigma_{\mathrm{S}}=0\right)$ the grass biomass and available soil resources are high (Figure 3.3). The grass biomass in the grass states abruptly goes to zero as interannual climate variability increases, while shrub biomass in the shrub state gradually increase as $\sigma_{\mathrm{G}}$ increases up to intermediate levels of interannual climate variability (Figure 3.3). Thus, as interannual climate variability increases, the probability of reaching a final state dominated by shrubs increases up to intermediate levels of interannual climate variability, while the probability of attainment of a grass state rapidly decreases (Figure 3.2). The decrease in vegetation biomass in the grass states is associated with an increase in erosion rates and with the consequent decrease in available soil resources as shown in Figure 3.3. Thus, when the final state is a grassland, vegetation biomass decreases with increasing levels of environmental variability. Conversely, whenever the final state of the system is dominated by shrubs, vegetation biomass 
remains relatively high and no major erosional losses of soil resources occur. Overall, the decrease in biomass is paralleled by a decrease in soil resources and vice versa (Figure 3.3).

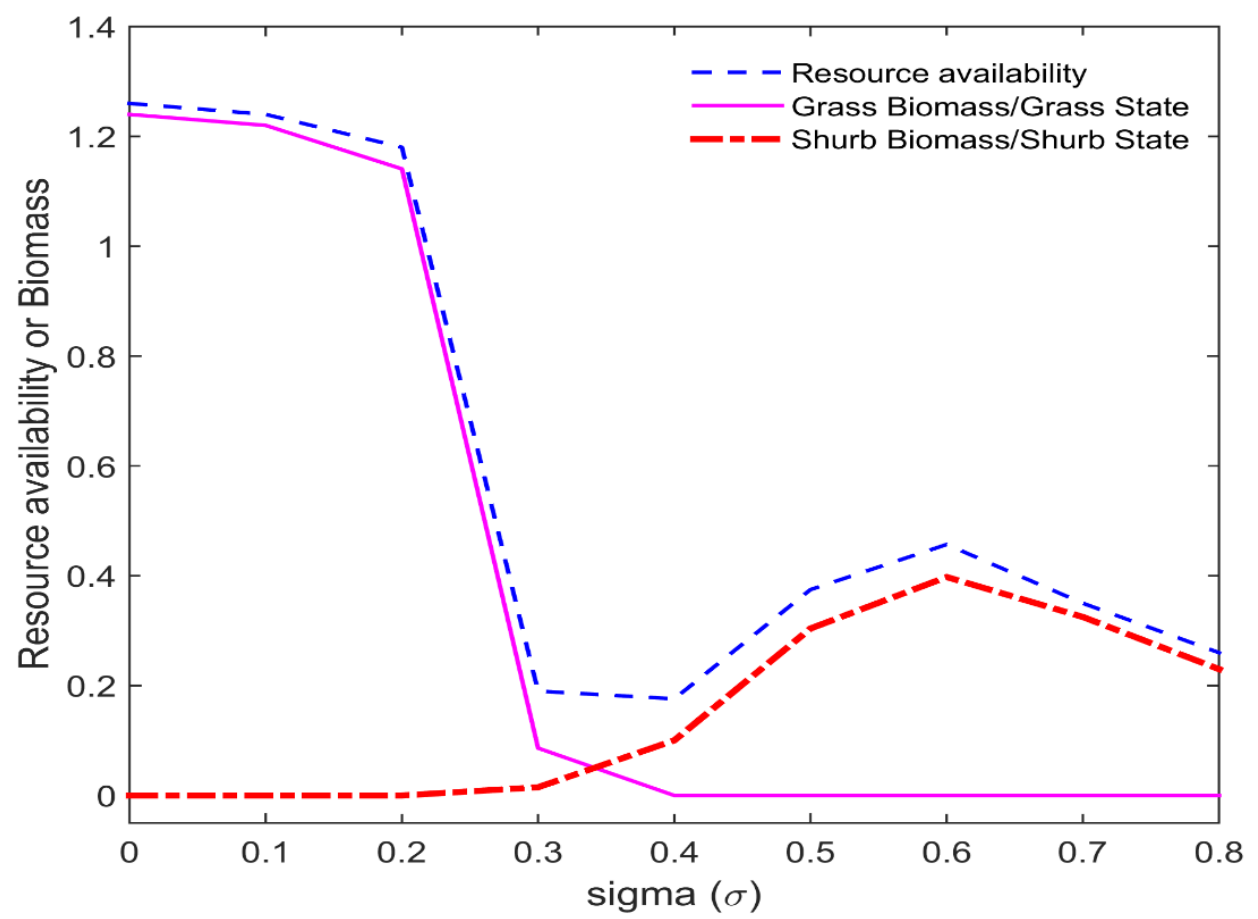

Figure 3-3: Resource concentration, shrub, and grass biomass at the final state ( $\mathrm{t}=3000)$ as a function of G (shown for the case of one time grass invasion). Same parameters as in Figure 3.2. Resource availability is calculated by equation 1 ; shrub and grass biomass are calculated as the mean shrub and grass biomass at final state $(\mathrm{t}=3000)$ over 1000 realizations of the stochastic process.

\subsection{Discussion}

This study develops a minimalist process-based model of coupled soil resource-vegetation dynamics to investigate changes in dryland vegetation associated with the invasion of desert shrublands by exotic grasses. Figure 3.1 describes the typical four stages of vegetation change characterized by different plant community composition, fire frequency, and ecosystem processes. Past studies have focused on the transitions from the state of shrubland to shrub/grass associations 
(States I to II) and from shrub/grass associations to the state of exotic grassland (States II to III) (e.g., D'Antonio and Vitousek, 1992; D'Antonio, 2000). Climate change studies predict an increase in the variability of precipitation across different scales in space and time (Nicholson 1980; Easterling et al., 2000; IPCC, 2013). This study investigates the transition from State III to the bare ground state (State IV) under the effect of interannual rainfall variability. While the impact of climate drivers on ecosystems is often investigated by examining only the effect of changes in mean climate conditions (e.g., Walther et al., 2002; Kljun et al., 2006), my modeling results show that grass invasions and interannual rainfall fluctuations could act in concert to induce the transition from States III to IV. Overall, this study highlights the importance of understanding the effect of rainfall variability on the potential ecosystem states.

This modeling study shows that, in the absence of climate fluctuations, invasive grasses (State III) replace the native shrubs (State I) (Figure 3.2). The factors favoring the replacement of shrubs by grasses include anthropogenic introduction (Dukes and Mooney, 1999), increase in atmospheric $\mathrm{CO}_{2}$ concentrations and temperature, and/or increase in soil resource availability (i.e., nitrogen) (Smith et al., 2000; Ziska et al., 2005; Sorte et al., 2013). Fluctuations in resource/rainfall availability could provide windows of opportunity in resource enrichment and thus species (i.e., grasses) with a high growth rate may quickly take up resources, change the disturbance regime (i.e., fires), and then invade or dominate the landscape (e.g., Davis et al., Davis and Melissa, 2001; Corbin and D'Antonio, 2004). However, opposite interactions may occur in arid environments where grasses exhibit slow growth rates (e.g., Teuling et al., 2010; Collins et al., 2012). Indeed, recent field studies at the Jornada Basin (New Mexico) show that increasing interannual rainfall fluctuations could favor shrubs over grasses (e.g., Gherardi and Sala, 2015 a b). 
The transition from state I to state III occurs through an intermediate state (State II) in which the introduction of a relatively continuous cover of exotic grasses favors the occurrence and spread of fires in the native shrubland (Figure 3.2) (D'Antonio, 2000). This intermediate state is not stable because grass establishment triggers the fire-vegetation feedback resulting in a stable grass cover (Grigulis et al., 2005; Keeley and Rundel, 2005). Such a transition from state I to state III could also occur in ecosystems where shrubs have deeper roots than grasses (Smit and Rethman, 2000; Hipondoka et al. 2003; Beckage et al. 2009) because in these dynamics shrub displacement by grass is not due to competition but to the ability of grasses to enhance fireinduced shrub mortality.

This study also shows that increases in climate fluctuations could reduce the probability for the system to reach a stable grassland state but increase the probability of converting to a stable shrubland state along with the probability of crashing to a bare soil state (Figure 3.2). This pattern corresponds with change in grass/shrub biomass and resource concentration as observed in Figure 3.3. The key in this modeled pattern is that the increasing frequency of drought under increased climate fluctuations may extend periods in which patches invaded by exotic grasses have no or low cover, especially when the grasses are susceptible to drought or lag in regrowth after drought (State IV) (e.g., Peake at al. 1979; Tilman and Haddi, 1992; Franklin et al., 2006). Plant mortality induced by moisture stress (a key determinant of the crash state) is expected to become more frequent in the future due to the combined effect of drought and temperature extremes (Adams et al., 2008; Volder et al., 2010). Thus, depending on their intensity (i.e., $\sigma_{\mathrm{G}}$ ), climate fluctuations could limit the establishment of exotic grasses before they are able to displace the native shrubs through the fire cycle, which leads to a stable shrubland (Figure 3.2). Alternatively, grass mortality could occur at a later stage after the fire cycle has killed all native shrubs. In this case, low or no 
vegetation cover (grasses and/or shrubs) leads to soil erosion and depletion of the seedbank and other soil resources (equation 1) (e.g., Peake et al 1979; Tilman and Haddi 1992; Franklin et al., 2006; Alvarez et al., 2012; Bhattachan et al., 2014). Erosive losses will then likely lead to lower vegetation productivity, thereby completing a positive feedback loop that slows down or prevents the regrowth of vegetation, including shrubs, even during wet years (e.g., Okin et al., 2009a). I also note that the regrowth of grass seeds during wet years can be limited by the short seed lifetime (e.g., Ellis, 1991; Bakker et al., 1996), which depends on seed size, shape, vertical distribution in the soil profile, and environmental conditions (e.g., temperature and soil moisture availability) (e.g., Ellis, 1991; Bakker et al., 1996; Bekker et al., 1998).

Experimental evidence (Supplementary Table 3.1) has been extensive in documenting a high sensitivity of perennial grasses in response to drought (e.g., Herbel, 1972; McClaran and Angell, 2006; Yao et al., 2006; Munson et al., 2012). Annual grasses have been found to be more sensitive to drought than perennial grass, likely because of their lower biomass per individual, small roots and, consequently, more limited access to soil water resources (e.g., Tilman and Haddi, 1992; Germino et al., 2016). In fact, invasions by exotic grasses, particularly annuals such as Bromus species leads to changes in litter, fire, and soil properties which appear to feedback to reinforce Bromus' dominance and further portends desertification under increased rainfall fluctuations (Germino et al., 2016). Overall, exotic grasses (perennial or annual) will not establish or grow significantly during a drought and are thus more sensitive to interannual rainfall variability and water deficits (Breman and Cissé, 1977; Mack and Pyke, 1983). The carrying capacity for grasses has therefore been modeled with the same mean but a higher variance than the carrying capacity for shrubs to reflect the higher susceptibility of exotic grasses to changes in biomass induced by interannual rainfall variability. Moreover, I also note that, in 
the case of annual grasses, there is a higher soil vulnerability to erosion because the soil surface is sheltered only by seasonal grass cover and weak root systems (e.g., Kort, 1998; Beyears, 2004). On the other hand, I also note that some invasive annuals and/or perennials may have unique physiological traits that allow them to tolerate rainfall variability and drought (e.g., Vaughn et al., 2011; Marshall et al., 2012).

Overall, this study shows how climate fluctuations may lead to qualitatively different ecosystem states that cannot be maintained in the deterministic counterpart of the process (i.e., with $\left.\sigma_{\mathrm{G}}=0\right)$. Random climate fluctuations are often associated with an effect of ecosystem disturbance, whereby environmental variability induces random oscillations of the state of the system around the stable states of the underlying deterministic dynamics. The results presented in this study show that the effect of random environmental variability on ecosystem dynamics may be more profound. Indeed it can induce the emergence of novel stable states that differ from those of the deterministic system. The ability of noise to induce new bifurcations and new dynamical behaviors has been documented and explained for a number of dynamical systems (Horsthemke and Lefever, 1984; Garcia-Ojalvo and Sancho, 1999). In the case of environmental dynamics it has been found that random environmental fluctuations may enhance biodiversity (Chesson, 2000; Benedetti-Cecchi et al., 2006; D'Odorico et al., 2008), form spatial patterns (D’Odorico et al., 2006b; D'Odorico et al., 2007), or stabilize the system around an unstable state of the underlying deterministic dynamics (D'Odorico et al., 2005). Known as noise-induced stability, this latter effect seems to occur in the case of Figure 3.2, where random fluctuations maintain the system in a state that would not be stable without environmental variability (Zeng and Neelin 2000).

The model captures the main features of these interactions and accounts for the different susceptibility of these two functional groups to fires and drought-induced mortality. I note, 
however, that the goal of this model is not to provide an accurate simulation of these complex dynamics, but to offer a conceptual framework for the analysis of the possible combined effects of grass invasions, fires, erosion, and climate fluctuations in arid and semiarid landscapes, particularly in terms of the production of a novel bare ecosystem state. Overall, the process based framework developed in this study has allowed us to generate new hypotheses on the effect of grass invasions and interannual rainfall fluctuations on changes in dryland vegetation. Future research will need to test these theories with field observations and manipulative experiments.

In summary, the modeling framework presented in this study shows how the combined effect of grass invasion, fire dynamics, erosion, and droughts may turn shrubland into exotic grasslands and, potentially, into vegetation- and resource-poor scablands. Although the potential for shrublands to be converted to invasive grasslands has been identified in the past, the possible emergence of the novel crash, or scabland, state under the effect of climate fluctuations is a possible hazard to be mitigated by the management of invasion-prone arid ecosystems. In the degradation mechanism described here biological invasions and climate change could act in concert to induce land degradation. 


\section{Woody plants-grass interactions as affected by hydraulic lift and interannual rainfall variability}

This chapter is adapted from Yu KL and D'Odorico P. (2014), Climate, vegetation, and soil controls on hydraulic redistribution in shallow tree roots, Advance in water resources, 66:70-80, and Yu KL and D'Odorico P. (2015), Hydraulic lift as a determinant of tree-grass coexistence on savannas, New Phytologist, 207:1038-1051, as well as Yu KL, Michael Saha, and D’Odorico P. The effects of interannual rainfall variability on tree-grass composition along Kalahari rainfall gradient, Ecosystems (in press).

\footnotetext{
Abstract

Savanna ecosystems are characterized by a mixed community of woody plants and grasses. The coexistence of woody plants and grasses in savannas is determined by a complex set of interacting factors that determine access to resources and demographic dynamics, under the effect of external drivers and vegetation feedbacks with the physical environment. To date, theoretical studies on the way facilitative interactions, resulting from hydraulic lift affect treegrass coexistence and the range of environmental conditions in which savannas are stable. are still missing. To this end I develop a new mechanistic model to investigate the role of hydraulic lift on the stability of tree-grass coexistence in savannas. This model accounts both for competition for soil water in the shallow soil and fire-induced disturbance. The modelling results show that hydraulic lift favors grasses, which scavenge the water lifted by woody plants. Thus, hydraulic lift expands (at the expense of woodlands) the range of environmental conditions in which savannas are stable. These results suggest that hydraulic lift could be an important mechanism responsible for the coexistence of woody plants and grasses in savannas.
} 
Precipitation variability has been predicted to increase in a warmer climate, and is expected to greatly affect plant growth, interspecies interactions, plant community composition, and other ecosystem processes. While previous studies have investigated the effect of intra-annual rainfall variability on plant growth and ecosystem dynamics, the impacts of interannual rainfall variability remain understudied. To this end, I used satellite data and a mechanistic model to explorestree-grass composition responses under interannual rainfall variability in arid to subhumid ecosystems along the Kalahari Transect in Southern Africa. Both satellite data and model results show that increasing interannual rainfall fluctuations favor deep rooted trees over shallow rooted grasses in drier environments (i.e., mean annual rainfall, MAP $<900-1000 \mathrm{~mm}$ ) but favor grasses over trees in wetter environments (i.e., MAP>900-1000 mm). I suggest that under increasing interannual rainfall fluctuations both direct effects on soil water availability and indirect effects mediated by tree-grass interactions and fire dynamics are expected to play an important role in determining changes in plant community composition.

\subsection{Introduction}

Characterized by a mixed community of woody plants and grasses, savanna ecosystems occur in many regions of the world, including Africa, Australia, South and North America, and Europe (Scholes and Walker, 1993; Scholes and Archer, 1997). The apparently stable coexistence of woody plants and grasses in savannas has often interested scientists because ecological theories indicate that, if two species are limited by the same resource, the species with lower resource requirement will displace the other (Tilman, 1982). Known as the "savanna question" (Sarmiento, 1984), the enigma of tree-grass coexistence has often been explained either by invoking spatial (Walter, 1971; Walker and Noy-Meir, 1982; Eagleson and Segarra, 1985) or temporal (Scholes and Archer, 1997; House et al., 2003) niche separation between the two 
functional groups (equilibrium theories), or by challenging the notion that savannas are systems in a stable equilibrium (disequilibrium theories) (Sankaran, 2004).

The spatial niche separation hypothesis assumes that grasses have preferential access to the shallow soil resources, while woody plants to deeper soil layers, an assumption often known as the "two layer hypothesis" (Walter, 1971). According to this theory, the long-term dynamics of vegetation reach an equilibrium characterized by a certain ratio between woody plants and grasses (Sankaran et al., 2004), while fluctuations in their relative abundance emerge in response to rainfall variation or disturbances such as fire and herbivory (Walker and Noy-Meir, 1982; van Langevelde et al., 2003). Walter's two layer hypothesis has been supported in some ecosystems (Knoop and Walker, 1985; Sala et al., 1989; Weltzin and McPherson, 1997) but not in others (Hipondoka et al., 2003; Sankaran et al., 2004; Beckage et al., 2009). Recent studies revisited this hypothesis and found that difference in resource uptake between woody plants and grasses could prevent competitive exclusion even with substantial root overlap (Holdo et al., 2013; Ward et al., 2013).

Disequilibrium theories propose that in the absence of disturbances the equilibrium state of the system exhibits either complete grass or woody plant cover (Archer, 1989; Sankaran et al., 2004). Disturbances such as fire and herbivory prevent the system from transitioning to its equilibrium state through ecological buffering (Jeltsch et al., 2000) or storage mechanisms (Higgins, 2000; Gardner, 2006). Disturbance-based models have gained favor in recent years, but typically they do not explicitly account for the role of competition between woody plants and grasses (Sankaran et al., 2004). It is also unclear whether conditions favorable for disturbanceinduced coexistence operate in all savannas (D’Odorico et al., 2006a; Scheiter and Higgins, 
2007) and whether tree-grass coexistence can be explained without invoking complete root separation or disturbances.

An analysis of the determinants of woody plant cover across more than 800 savanna sites in Africa (Sankaran et al., 2005) suggested that coexistence mechanisms based on resource competition tend to occur in dry savannas (mean annual precipitation, MAP $<650 \mathrm{~mm}$ ), while the disturbance-based mechanism is typical of wet savannas (MAP> $650 \mathrm{~mm}$ ). Recent studies suggest that low resource levels (predominately soil water) in dry savannas limit woody cover and thus permit grasses to persist in the system (Sankaran et al., 2005; Higgins et al., 2010). In contrast, in more mesic environments, light limitations play a role because woody plants develop higher leaf area thereby limiting light availability to grasses (Dohn et al., 2013; Moustakas et al., 2013). Therefore, in the absence of disturbances the system would turn into a woodland (Sankaran et al., 2005). Similar results were obtained by recent savanna models that account for woody root (i.e., water) and shoot (i.e., light) competition separately (Scheiter and Higgins, 2007; Higgins et al., 2010).

An alternative mechanism for the coexistence of woody plants and grasses that has not been investigated before is related to the phenomenon of hydraulic lift (HL) (Figure 4.1b). HL transports soil water available at depth to the (drier) shallow soil through the plant roots during nighttime (Dawson, 1993; Caldwell et al., 1998). HL is expected to favor grasses and/or tree seedlings, which are found to be capable of scavenging the water lifted by trees (Dawson, 1993; Zou et al., 2005; Scott et al., 2008; Quijano et al., 2012). Past studies suggest that HL contributes to the facilitation of overstory woody plants on understory plants (shrubs or grasses) (Riginos et al., 2009; Quijano et al. 2012; Dohn et al., 2013; Moustakas et al., 2013), but theoretical work investigating in detail the role of $\mathrm{HL}$ in determining tree-grass coexistence in savannas and 
altering the range of environmental conditions in which savannas are stable is still missing (Ludwig et al., 2003). HL benefits grass growth and is thus expected to expand - with respect to the case with no HL - the range of environmental conditions in which grasslands or savannas can occur. While model simulations have shown that HL reduces water stress in grasses (Yu and D’Odorico, 2014b), it remains unclear how it affects their interactions with woody plants and the overall dynamics of vegetation.

The woody plants-grass interactions in savannas is also greatly affected by interannual rainfall variability. Unlike the effects of intra-annual rainfall variability, research on the effects of interannual rainfall variability is strongly limited by the availability of long-term field observational data and technical capability to measure all relevant variables (Fatichi and Ivanov, 2014; Ng et al., 2015). Long-term field campaigns have seldom addressed the impacts of interannual rainfall variability on ecosystem dynamics (Munson et al., 2012; Collins and Xia, 2015; but see Knapp et al., 2002). Indeed, in field experiments with no rainfall manipulations changes in rainfall variability may often be associated with changes in mean rainfall, thus making it difficult to differentiate the effects of changes in rainfall variability from those of changes in mean rainfall (Hsu et al., 2012; Reyer et al., 2013). To overcome these limitations, process-based models - possibly integrated with field or satellite data - could offer alternative approaches to gain a mechanistic understanding of the ecological impacts of trends in rainfall variability (Reyer et al., 2013; Fatichi and Ivanov, 2014).

How a functional group (i.e., trees) responds to an increase in interanual rainfall variability depends on the trade-off between wet and dry years (Holmgren et al., 2013) as well as on its competitive relationship with other functional groups (grasses) and associated disturbance regime (i.e., fires) (Williams and Albertson, 2006; Reyer et al., 2013; Zeppel et al., 2014). A 
dynamic soil moisture model has been used to show the effect of grass dynamics on tree response to increasing interannual rainfall variability (Scanlon and Albertson, 2003). FernandezIllescas and Rodriguez-Iturbe (2003) developed a hierarchical competition-colonization model in which dynamic water stress affects the tree/grass competition-colonization relationship; these authors used this model to investigate the impact of interannual rainfall fluctuations on tree/grass associations. Both of these models, however, ignored the roles of fires. Indeed, grasses typically have relatively high growth rates especially in wet environments (Teuling et al., 2010; Collins et al., 2012; Xu et al., 2015) and thus may more quickly take up soil water or other resources, thereby more effectively competing with trees in wet years. The competitive advantage of grasses in wet years could enhance fire occurrences, thereby increasing tree mortality (Bond et al., 2008; Ratajczak et al., 2014); as a result, the increase in interannual rainfall variability is expected to favor grasses at the expenses of trees.

To my knowledge, only few studies have modeled the impact of interannual rainfall fluctuations in savannas accounting for both water competition and fires (Williams and Albertson, 2006). Key to the understanding of vegetation response to changes in rainfall variability in water limited ecosystems is an adequate representation of the soil water balance (Porporato et al., 2002). In fact, the increase in rainfall intensity (with the total rainfall amount remaining constant) has been found to lead to shrub encroachment because of increased drainage into the deeper soil layer where trees with deeper roots can have exclusive access to soil water (Kulmatiski and Beard, 2013a b). Recent studies suggest that the increase in interannual rainfall variability may also increase soil water availability in the deep soil, thereby favoring deep rooted over shallow rooted species (Sala et al., 2015; Gherardi and Sala, 2015 a b). It remains unclear how the trait-based trade off (i.e., higher growth rate but shallower roots in grasses than trees) 
affects the response of tree-grass associations to increasing interannual rainfall variability along a rainfall gradient.

I first develop a new mechanistic model to investigate the impact of $\mathrm{HL}$ on tree-grass interactions and the overall dynamics of savanna ecosystems. This model accounts both for competition for soil water in the shallow soil and fire-induced disturbance. I then adapt the mechanistic model to investigate the response of tree-grass composition to increasing interannual rainfall variability in arid to sub-humid ecosystems along the Kalahari Transect in Southern Africa. By clarifying the role of HL and interannual rainfall variability in woody plants-grass interactions, this study contributes to a better understanding of savanna susceptibility to changes in climate and disturbance regimes (i.e., fires).

\subsection{Methods}

\subsubsection{Modelling framework for the role of hydraulic lift}

I develop a mechanistic model (hereafter called model 1) to investigate the role of hydraulic redistribution (HR) on the interactions between woody plants and grasses and the dynamics of savanna ecosystems. HR is the transport of soil water either from wetter deep soil to drier shallow soil (hydraulic lift, HL) or from wetter shallow soil to drier deep soil (hydraulic descent). HR is driven by soil hydraulic gradients, occurs through the plant roots, and takes place at night when no transpiration occurs (Figure 4.1b). Consistent with other studies (Ryel et al., 2002; Lee et al., 2005; Yu and D'Odorico, 2014b), the net direction and magnitude of HR is quantified by a model which simulates soil moisture dynamics in two soil layers and accounts for flows between them due to drainage and HR. Woody plants are assumed to have roots in both layers and perform HR (Quijano et al., 2012; Yu and D’Odorico, 2014b); grasses are assumed to 
have roots only in the shallow soil layer (Kulmatiski and Beard, 2013a b; Yu and D'Odorico, 2014b).
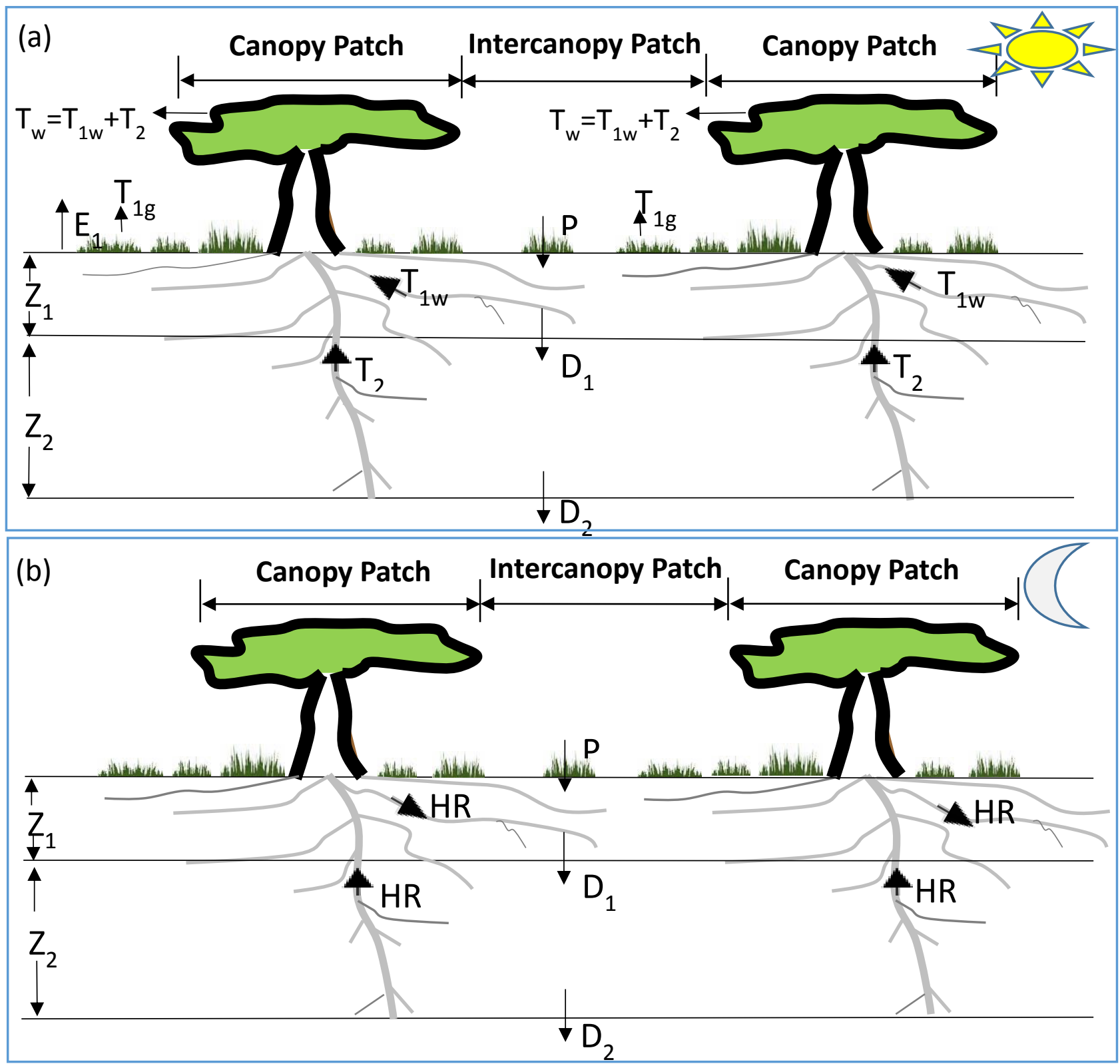

Figure 4-1: A schematic representation of soil water budget during the day (a) and during the night (b). This schematic representation also shows the typical patchy distribution of vegetation with areas covered by woody plant canopies, or "canopy patches" and "intercanopy patches" with sparse grasses and bare soil. The readers can refer to Supplementary Table 4.1 for the meaning of each variable. 
Biomass dynamics of woody plants and grasses are represented by logistic growth equations which account for biomass loss from senescence and litter fall and/or disturbance (fires). Biomass loss resulting from senescence and little fall will be decomposed and thus senescence and little fall is modeled together as a decomposition process for the sake of simplicity. HR changes the soil resource (water) availability in the two soil layers and thus is expected to affect the interactions between woody plants and grasses (Figure 4.1b). The model uses a stochastic representation of rainfall and soil moisture dynamics (Rodriguez-Iturbe et al., 1999a) and deterministic laws to express the interactions and dynamics of woody plants and grasses, without accounting for the randomness of demographic growth in woody plants (Higgins, 2000; Gardner, 2006), the interannual variability of rainfall (van Wijk and Rodriguez-Iturbe, 2002), and the stochastic nature of fire (D’Odorico et al., 2006a). The model is not spatially explicit and thus it does not explicitly represent the spatial interactions among woody plants and grasses (Jeltsch et al., 1996, Rodriguez-Iturbe et al., 1999b; van Wijk and Rodriguez-Iturbe, 2002). The model, however, accounts for the impact on the soil water balance of spatial heterogeneities associated with the mosaic-like structure of the landscape and - as it is shown below - uses a lumped approach to relate patch-scale to landscape-scale variables (Figure 4.1a, b). Consistent with other studies (van Wijk and Rodriguez-IturbeI, 2002; van Langevelde et al., 2003), woody plants and grasses are assumed to compete for soil water resources in the shallow soil layer while woody plants have exclusive access to the soil water resources in the deep soil layer. I also assume that woody plants have rapid root growth in early stages and thus can develop their exclusive niche in the deep soil layer relatively early in their lifetime (Brown and Archer, 1990; Bragg et al., 1993; Scholes and Archer, 1997). Therefore the model is not well suited to investigate the effect of demographic bottlenecks on tree-grass coexistence (Higgins, 2000). Woody plants are assumed 
to exert a highly asymmetric light competition on grasses (Scheiter and Higgins, 2007; Higgins et al., 2010), particularly in wet environments where grass growth is limited by light availability under woody plant canopies (Dohn et al., 2013; Moustakas et al., 2013).

\subsubsection{Modelling framework for the role of interannual rainfall variability}

The model developed to investigate the role of hydraulic redistribution is adapted to investigate the response of trees or grasses alone and tree-grass associations to increasing interannual rainfall variability along a rainfall gradient. The model (hereafter called model 2) simulates soil moisture dynamics in two soil layers and accounts for flows between them due to drainage but without the effects of hydraulic redistribution. The model simulates the biomass dynamics of trees and grasses as a logistic growth coupled with the soil water balance and accounts for biomass loss from senescence, litter fall and disturbance (fires). Trees are assumed to have roots in both layers while grasses are assumed to have roots only in the shallow soil layer (van Wijk and Rodriguez-Iturbe, 2002; Van Langevelde et al., 2003; Kulmatiski and Beard, 2013a b; Yu and D’Odorico, $2015 \mathrm{a}$ b). Thus, trees and grasses are assumed to compete for soil water resources in the shallow soil layer, while trees can exclusively access the soil water resources in the deep soil layer. While in some savannas grasses could have roots as deep as trees (Sankaran et al., 2004; Beckage et al., 2009), here I consider the case in which tree roots span a much deeper soil column than grasses (Walter, 1971; Kulmatiski and Beard, 2013 a b; Holdo and Nippert, 2015). An increase in interannual rainfall variability changes the soil moisture profile in the root zone and thus is expected to affect the interactions between trees and grasses. Rainfall and soil moisture dynamics are represented as stochastic processes (Rodriguez-Iturbe et al., 1999a); interannual rainfall fluctuations are accounted for by a two-parameter gamma distribution (D’Odorico et al., 2000; Porporato et al., 2006). Deterministic laws are used to 
express the interactions and dynamics of trees and grasses, without accounting for the randomness of demographic growth in trees (Higgins et al., 2000; Gardner, 2006), and the stochastic nature of fire (D'Odorico et al., 2006a). The model does not explicitly represent the spatial interactions among trees and grasses (Jeltsch et al., 1996; van Wijk and Rodriguez-Iturbe, 2002) and thus uses a lumped approach to simulate dynamics of trees and grasses. Since model 2 is adapted from model 1 , in this chapter details of model 1 including soil water balance and vegetation dynamics are provided while the reader is referred to $\mathrm{Yu}$ and D'Odorico (in press) for details of the model 2.

\subsubsection{Soil moisture dynamics}

Soil moisture dynamics in the two soil layers are modeled at the landscape scale by two coupled equations (Figure 4.1a, b)

$$
\mathrm{nZ}_{1} \frac{\mathrm{dS}_{1}}{\mathrm{dt}}=\mathrm{P}-\mathrm{ET}_{1}-\mathrm{D}_{1}+\mathrm{HR} \quad \mathrm{Eqn} 1 \mathrm{a}
$$

and

$$
\mathrm{nZ}_{2} \frac{\mathrm{dS_{2 }}}{\mathrm{dt}}=\mathrm{D}_{1}-\mathrm{T}_{2}-\mathrm{D}_{2}-\mathrm{HR} \quad \text { Eqn } 1 \mathrm{~b}
$$

where the subscripts 1 and 2 refer to the shallow and deep soil layer, respectively; $\mathrm{n}$ is the soil porosity, $\mathrm{Z}_{1}$ and $\mathrm{Z}_{2}$ the soil layer thickness $(\mathrm{mm}), \mathrm{S}_{1}$ and $\mathrm{S}_{2}$ the relative soil moisture $\left(0<\mathrm{S}_{1}\right.$, $\left.\mathrm{S}_{2} \leq 1\right), \mathrm{P}$ the rate of rainfall infiltration into the top soil layer $\left(\mathrm{mm} \mathrm{d}^{-1}\right), \mathrm{ET}_{1}$ and $\mathrm{T}_{2}$ the soil moisture losses from each soil layer due to evapotranspiration $\left(\mathrm{mm} \mathrm{d}^{-1}\right), \mathrm{D}_{1}$ and $\mathrm{D}_{2}$ the drainage rates $\left(\mathrm{mm} \mathrm{d}^{-1}\right)$, and $\mathrm{HR}$ the hydraulic redistribution $\left(\mathrm{mm} \mathrm{d}^{-1}\right)$. Note that in these equations all water fluxes are expressed at the landscape scale. Positive values of HR indicate "hydraulic lift" (i.e., upward hydraulic redistribution), while negative values of HR indicate "hydraulic descent" 
(i.e., downward hydraulic redistribution). Runoff occurs when the surface layer is saturated (i.e., $\left.S_{1}=1\right)$. Drainage is assumed to be driven only by gravity and is expressed as

$$
\mathrm{D}=\frac{\mathrm{K}_{\mathrm{s}} \exp \left(\beta\left(\mathrm{S}-\mathrm{S}_{\mathrm{fc}}\right)-1\right)}{\exp \left(\beta\left(1-\mathrm{S}_{\mathrm{fc}}\right)-1\right)} \quad \text { Eqn 1c }
$$

where $K_{s}$ is the soil saturated hydraulic conductivity $\left(\mathrm{mm} \mathrm{h}^{-1}\right), \beta$ a coefficient, $S$ the relative soil moisture, $\mathrm{S}_{\mathrm{fc}}$ the field capacity (Laio et al., 2001).

\subsubsection{Evapotranspiration by woody plants and grasses}

Potential evapotranspiration of woody plants $\left(\mathrm{PET}_{\mathrm{w}}\right)$ and grasses $\left(\mathrm{PET}_{\mathrm{g}}\right)$ is assumed to depend on shortwave solar radiation available (Yu and D'Odorico, 2014 a). Shortwave radiation exponentially decays through the plant canopy (Beer's law). Following other studies (Caylor et al., 2005; Yu and D’Odorico, $2014 \mathrm{a}$ ), $\mathrm{PET}_{\mathrm{w}}$ and $\mathrm{PET}_{\mathrm{g}}$ are determined as $\mathrm{PET}_{\mathrm{w}}=$ PET $\left[1-\exp \left(-\mathrm{e}_{\mathrm{s}} \mathrm{LAI}_{\mathrm{lw}}\right)\right]$ and $\mathrm{PET}_{\mathrm{g}}=\mathrm{PET} \exp \left(-\mathrm{e}_{\mathrm{s}} \mathrm{LAI}_{\mathrm{lw}}\right)$, respectively, where PET is the total potential evapotranspiration $\left(\mathrm{mm} \mathrm{d}^{-1}\right), \mathrm{e}_{\mathrm{s}}$ the extinction coefficient of shortwave radiation, and $\mathrm{LAI}_{\mathrm{lw}}$ woody plant leaf area index expressed at the landscape scale $\left(\mathrm{m}^{2} \mathrm{~m}^{-2}\right)$, calculated as explained below. Potential evapotranspiration of woody plants $\left(\mathrm{PET}_{\mathrm{w}}\right)$ is partitioned into potential evapotranspiration for the shallow soil layer $\left(\mathrm{PET}_{1 \mathrm{w}}\right)$ and potential transpiration for the deep soil layer $\left(\mathrm{PT}_{2 \mathrm{w}}\right)$, i.e., $\mathrm{PET}_{1 \mathrm{w}}=\mathrm{PET}_{\mathrm{w}} \mathrm{Z}_{1} \mathrm{~S}_{1} /\left(\mathrm{Z}_{1} \mathrm{~S}_{1}+\mathrm{Z}_{2} \mathrm{~S}_{2}\right)$ and $\mathrm{PT}_{2 \mathrm{w}}=\mathrm{PET}_{\mathrm{w}} \mathrm{Z}_{2} \mathrm{~S}_{2} /$ $\left(\mathrm{Z}_{1} \mathrm{~S}_{1}+\mathrm{Z}_{2} \mathrm{~S}_{2}\right.$ ) (Yu and D'Odorico, 2014a). In general, the transpiration model should account also for seasonal changes in plant hydraulic conductivity; this effect, however, is here not accounted for because I prefer to limit the complexity of the model. The actual transpiration by plants is then determined by accounting for the soil water availability (Rodriguez-Iturbe et al., 1999a; Guswa et al., 2002, 2004). Thus the actual evapotranspiration for grasses $\left(\mathrm{ET}_{1 \mathrm{~g}}\right)$ is 
calculated as $\mathrm{ET}_{1 \mathrm{~g}}=\mathrm{PET}_{\mathrm{g}} \tau\left(\mathrm{S}_{1}\right)$, where $\tau\left(\mathrm{S}_{1}\right)$ is a function expressing the reduction in evapotranspiration resulting from soil moisture limitations. The actual evapotranspiration of woody plants is contributed by root uptake from the shallow soil layer $\left(\mathrm{ET}_{1 \mathrm{w}}\right)$ and root uptake from the deep soil layer $\left(\mathrm{T}_{2}\right)$, which depend on the root profile as $\mathrm{ET}_{1 \mathrm{w}}=\mathrm{PET}_{1 \mathrm{w}} \tau\left(\mathrm{S}_{1}\right) \alpha_{1}$ and $\mathrm{T}_{2}=\mathrm{PT}_{2 \mathrm{w}} \tau\left(\mathrm{S}_{2}\right) \alpha_{2}$, where $\alpha_{1}$ and $\alpha_{2}$ are cumulated (and normalized, $\alpha_{1}+\alpha_{2}=1$ ) woody root densities in the shallow and the deep soil layers, respectively.

\subsubsection{Hydraulic redistribution}

Hydraulic redistribution is driven by a water potential gradient and is determined as $\mathrm{HR}=$ $\mathrm{R}_{\mathrm{e}} \mathrm{C}_{\mathrm{rmax}}\left(\Psi_{\mathrm{s} 2}-\Psi_{\mathrm{s} 1}\right) \min \left(\alpha_{1}, \alpha_{2}\right) \mathrm{f}_{\mathrm{r}}$ without considering gravitational potential (Ryel et al., 2002; Lee et al., 2005; Yu and D'Odorico, 2014b), where $\mathrm{R}_{\mathrm{e}}$ is a factor reducing root hydraulic conductance and accounting for soil water limitation, $\mathrm{C}_{\mathrm{rmax}}$ the maximum root hydraulic conductance of the entire active root system $\left(\mathrm{mm} \mathrm{MPa}^{-1} \mathrm{~h}^{-1}\right), \Psi_{\mathrm{s} 2}$ and $\Psi_{\mathrm{s} 1}$ the soil water potential (MPa) in the deep and the shallow soil layer, respectively, and $f_{r}$ the fractional root cover of woody plants at the landscape scale (\%), calculated as explained below. $\Psi$ is a function of soil moisture expressed as $\Psi=\Psi_{\mathrm{S}} \times \mathrm{S}^{-\mathrm{d}}$, where $\Psi$ is soil water potential, $\mathrm{S}$ soil moisture, while $\Psi_{\mathrm{S}}$ and $\mathrm{d}$ are experimentally derived parameters (Clapp and Hornberger, 1978). $\mathrm{f}_{\mathrm{r}}$ is used to quantify HR expressed at the landscape scale. Note that the dynamics of soil moisture in the shallow $\left(S_{1}\right)$ and deep $\left(S_{2}\right)$ soil layers are fully coupled, i.e., $S_{1}$ and $S_{2}$ depend on HR, which in turn depends on the water potential gradients between the two soil layers.

Following Caylor et al (2006) I represent the landscape as a mosaic of "canopy" and "between canopy" areas resulting from a two-dimensional Poisson distribution of individual woody plants 
(with circular footprint). According to Caylor et al (2006), the fractional canopy cover of woody plants $\left(\mathrm{f}_{\mathrm{c}}\right)$ is determined as:

$$
\mathrm{f}_{\mathrm{c}}=1-\exp ^{-\pi \mathrm{i}_{\mathrm{w}} \mathrm{u}_{\mathrm{c}}{ }^{2}} \quad \text { Eqn } 2 \mathrm{a}
$$

where $\mathrm{i}_{\mathrm{w}}$ is the number of woody plant individuals per unit area (individuals $\mathrm{m}^{-2}$ ) and $\mathrm{u}_{\mathrm{c}}$ the canopy radius of an individual of woody plant canopy (m). Likewise, I express the fractional root cover of woody plants $\left(f_{r}\right)$ as:

$$
\mathrm{f}_{\mathrm{r}}=1-\exp ^{-\pi \mathrm{i}_{\mathrm{w}} \mathrm{u}_{\mathrm{r}}{ }^{2}} \quad \text { Eqn } 2 \mathrm{~b}
$$

where $u_{r}$ is the radius of the footprint of root zone of an individual of woody plant (m). Rearranging the Eqn 2(a) and Eqn 2(b), I have:

$$
\mathrm{f}_{\mathrm{r}}=1-\left(1-\mathrm{f}_{\mathrm{c}}\right)^{\left(\frac{\mathrm{u}_{\mathrm{r}}}{\mathrm{u}_{\mathrm{c}}}\right)^{2}} \quad \text { Eqn 2c }
$$

I express the biomass of woody plants at the landscape scale $\left(\mathrm{W}_{\mathrm{l}}, \mathrm{kg} \mathrm{m}^{-2}\right)$ as:

$$
\mathrm{W}_{\mathrm{l}}=\mathrm{W}_{\mathrm{p}} \times \mathrm{i}_{\mathrm{w}} \quad \text { Eqn } 2 \mathrm{~d}
$$

where $\mathrm{W}_{\mathrm{p}}$ is the biomass of an individual woody plant canopy $\left(\mathrm{kg}_{\mathrm{ind}}{ }^{-1}\right)$. Rearranging Eqn 2(a) and Eqn 2(d), I obtain:

$$
\mathrm{W}_{\mathrm{l}}=\frac{\mathrm{W}_{\mathrm{p}}}{\pi \mathrm{u}_{\mathrm{c}}^{2}} \log \frac{1}{1-\mathrm{f}_{\mathrm{c}}} \quad \text { Eqn } 2 \mathrm{e}
$$

4.2.6 Biomass dynamics of woody plants and grasses

The dynamics of grass biomass at the landscape scale $\left(\mathrm{G}_{1}, \mathrm{~kg} \mathrm{~m}^{-2}\right)$ are represented as a logistic growth with a mortality term accounting for decomposition:

$$
\frac{\mathrm{dG}_{1}}{\mathrm{dt}}=\mathrm{gg}_{\mathrm{g}} \mathrm{G}_{\mathrm{l}}\left(\mathrm{V}_{1}-\mathrm{G}_{\mathrm{l}}-\gamma \mathrm{W}_{\mathrm{l}}\right)-\mathrm{m}_{\mathrm{G}} \mathrm{G}_{\mathrm{l}} \quad \text { Eqn } 3 \mathrm{a}
$$


where $g_{g}$ is the grass growth coefficient, $V_{1}$ the part of vegetation carrying capacity contributed by soil moisture in the shallow soil layer $\left(\mathrm{kg} \mathrm{m}^{-2}\right), \gamma$ the fraction of woody plants biomass relying on the shallow soil layer, and $\mathrm{m}_{\mathrm{G}}$ the grass decomposition coefficient. Grass decomposition removes each year a portion of biomass after the growing season. Grasses are assumed to quickly recover from fires (Russell-Smith et al., 2001) and thus I do not include a fire mortality term in the grass dynamics equation. $\gamma$ is determined as:

$$
\gamma=\frac{\mathrm{ET}_{1 \mathrm{w}}}{\mathrm{ET}_{1 \mathrm{w}}+\mathrm{T}_{2}} \quad \text { Eqn } 3 \mathrm{~b}
$$

The term $\gamma \mathrm{W}_{\mathrm{l}}$ here is used to express the belowground competitive effect of woody plants on grasses for soil water in the shallow soil layer. A high value of $\gamma W_{l}$ is expected to lead to competitive exclusion of grasses. The ratio between woody root density in the shallow soil layer $\left(\alpha_{1}\right)$ and the deep soil layer $\left(\alpha_{2}\right)$ greatly affects $\gamma$. Higher values of $\alpha_{1} / \alpha_{2}$ correspond to situations with more root overlapping between woody plants and grasses in the shallow soil layer (because $\alpha_{1}+\alpha_{2}=1$ ).

The dynamics of woody plant biomass at the landscape scale $\left(\mathrm{W}_{\mathrm{l}}\right)$ are modeled as a logistic growth with mortality terms accounting for fire and decomposition:

$$
\frac{d W_{1}}{d t}=g_{w} W_{l}\left[\left(V_{1}-G_{l}-\gamma W_{l}\right)+V_{2}-(1-\gamma) W_{l}\right]-m_{W} W_{l}-k_{\eta} W_{l} \quad \text { Eqn } 3 c
$$

where $g_{\mathrm{w}}$ is the growth coefficient of woody plants, $\mathrm{V}_{2}$ the portion of vegetation carrying capacity contributed by the deep soil layer $\left(\mathrm{kg} \mathrm{m}^{-2}\right), \mathrm{m}_{\mathrm{W}}$ the decomposition coefficient of woody plants, $\mathrm{k}$ a parameter determining the death rate of woody plants by fires, and $\eta$ the fire frequency depending on grass biomass (van Wilgen et al., 2000). Woody plant decomposition is modeled as a process that removes a proportion of biomass after the growing season in each 
year. In agreement with other studies (Walker and Noy-Meir, 1982; van Langevelde et al., 2003), fires are modeled as a process that continuously removes the biomass of woody plants.

\subsubsection{Landscape scale dynamics}

I first examine the resource-based competition mechanism of coexistence between woody plants and grasses in relatively dry savannas (MAP $<650 \mathrm{~mm}$ ). To this end, I consider the case with no woody biomass losses from fires $(\mathrm{k \eta}=0)$ and investigate how the coexistence of woody plants and grasses may occur in dry savannas in the absence of fires (Sankaran et al., 2004, 2005; Scheiter and Higgins, 2007; Higgins et al., 2010). In dry savannas I also do not account for light limitation in grass growth because the woody plant cover is relatively low and thus light limitation in grasses is insignificant, in agreement with other studies (Scheiter and Higgins 2007; Higgins et al., 2010; Yu and D’Odorico 2014a). I then examine the disturbance-based mechanism of coexistence between woody plants and grasses in wet savannas (MAP>650 mm), and account for light limitation in grasses and woody biomass loss from fires.

To this end, I use Beer's law to calculate solar irradiance under woody plant canopies as a function of the leaf area index $\left(\overline{\mathrm{LAI}}_{\mathrm{w}}, \mathrm{m}^{2} \mathrm{~m}^{-2}\right)$ of woody plants (Scheiter and Higgins, 2007; Yu and D'Odorico, 2014a). I then account for the impact of reduced light availability (under woody plant canopies) on grass growth through a light limitation coefficient (l) calculated as the ratio of actual to light saturation net photosynthesis rate, based on leaf-level physiological measurements (O'Halloran, 2007). Thus, the landscape scale grass biomass with light limitation $\left(\mathrm{G}_{\mathrm{ll}}\right)$ is calculated as:

$$
\mathrm{G}_{\mathrm{ll}}=\mathrm{l} \times \mathrm{G}_{\mathrm{l}} \quad \text { Eqn 4a }
$$


Note that $G_{l l}$ replaces $G_{l}$ calculated with Eqn 3(a); $G_{l l}$ expresses the landscape scale grass biomass when light limitation is accounted for in wet savannas (MAP> $650 \mathrm{~mm}$ ). $\mathrm{G}_{\mathrm{ll}}$ is then used in Eqn 3(c) in place of $G_{l}$ when the landscape scale dynamics of woody plant biomass are simulated. The average leaf area index $\left(\overline{\mathrm{LAI}}_{\mathrm{w}}, \mathrm{m}^{2} \mathrm{~m}^{-2}\right)$ of woody land patches is one of the crucial parameters in the calculation of 1 . Following Yu and D'Odorico (2014a), $\overline{\mathrm{LAI}}_{\mathrm{w}}$ is expressed as:

$$
\overline{\mathrm{LAI}}_{\mathrm{w}}=-\frac{\log \left(1-\mathrm{f}_{\mathrm{c}}\right)}{\mathrm{f}_{\mathrm{c}}} \text { LAI } \quad \text { Eqn } 4 \mathrm{~b}
$$

where LAI is the leaf area index of an individual woody plant canopy $\left(\mathrm{m}^{2} \mathrm{~m}^{-2}\right)$. The fraction on the right-hand side of Eqn 4(b) accounts for the average number of overlapping canopies existing at any point in the landscape (Caylor et al., 2006). Consistent with other studies (Scheiter and Higgins, 2007), l decreases exponentially with the woody plant cover, as implied by Beer's Law. Woody plant leaf area index at the landscape scale $\left(\mathrm{LAI}_{l \mathrm{w}}\right)$ is expressed as $\mathrm{LAI}_{l \mathrm{w}}=\overline{\mathrm{LAI}}_{\mathrm{w}} \mathrm{f}_{\mathrm{c}}$.

\subsubsection{Case study: Kalahari Transect in Southern Africa}

The model is parameterized for the case of the Kalahari Transect in Southern Africa. This region exhibits relatively homogenous soils (sand) along a rainfall gradient, and provides excellent opportunities to investigate changes in plant community composition associated with different rainfall regimes (Koch et al., 1995). The rainy season lasts 210 days from October to May (about $95 \%$ of mean annual rainfall (MAP)) (Bhattachan et al., 2012). Long-term daily rainfall data for some areas of the Kalahari Transect is lacked and thus precipitation is modeled as a sequence of intermittent rainfall events occurring as a marked Poisson process with average rainfall frequency, $\lambda$, (events per day). Each storm size $(\mathrm{mm})$ is modeled as an exponentially distributed random variable with mean, h , (mm per event) (Rodriguez-Iturbe et al., 1999a). This stochastic 
rainfall model has been found to be an appropriate description of the distribution of growing season rainfall across the Kalahari Transect (Porporato et al., 2003; Caylor et al., 2005). Mean rainfall depth (h) is relatively uniform across the Kalahari rainfall gradient and is $10 \mathrm{~mm}$ per event, consistent with other studies (Porporato et al., 2003; Caylor et al., 2005; Bhattachan et al., 2012). Following Porporato et al (2003), average rainfall frequency $(\lambda)$ is limited to vary from $0.1 \mathrm{~d}^{-1}$ to $0.5 \mathrm{~d}^{-1}$ across the Kalahari rainfall gradient. Thus, this allows mean rainfall depth (h) to be less $10 \mathrm{~mm}$ per event in very dry environments (i.e., MAP $<220 \mathrm{~mm}$ ) but to be more than 10 per event in very wet environments (i.e., MAP>1110 mm).

The quantification of vegetation carrying capacity in the shallow $\left(\mathrm{V}_{1}\right)$ and deep $\left(\mathrm{V}_{2}\right)$ soil layers is based on the data from Sankaran et al (2005) who found that woody plants are completely absent at MAP $<100 \mathrm{~mm}$ in a number of savanna sites in Africa. Because in these very dry environments where deep soil moisture is low (hence, $V_{2} \approx 0$ ), woody plants would need to rely on shallow soil moisture. Grasses, however, have physiological and life form traits that make them more resistant to drought and are therefore better competitors than woody plants for shallow soil water (Noy-Meir, 1973). This explains why trees are completely absent from areas with MAP $<100 \mathrm{~mm}$ as observed by Sankaran et al (2005). Here I express $V_{1}$ and $V_{2}$ as:

$\mathrm{V}_{1}=\mathrm{C}_{0} \mathrm{Z}_{1} \mathrm{~S}_{1} \quad$ Eqn 5a

and

$\mathrm{V}_{2}=\left\{\begin{array}{cc}\mathrm{C}_{0} \mathrm{Z}_{2}\left(\mathrm{~S}_{2}-\mathrm{S}_{2 \mathrm{w}}\right), \mathrm{MAP} \geq 100 \mathrm{~mm} \\ 0, & \text { MAP }<100 \mathrm{~mm}\end{array} \quad\right.$ Eqn $5 \mathrm{~b}$

where $\mathrm{C}_{0}\left(\mathrm{~kg} \mathrm{~m}^{-2} \mathrm{~mm}^{-1}\right)$ is a coefficient converting the soil water resources into vegetation carrying capacity, $S_{2 w}$ the wilting point for woody plants, $S_{1}$ and $S_{2}$ relative soil moisture in the 
shallow and deep soil layers, respectively. Note that in the simulations $V_{1}$ and $V_{2}$ are continuously updated as a function of changing values of $S_{1}$ and $S_{2} . C_{0}$ is determined by fitting model parameters to the field data in a way that the woody plant biomass at the landscape scale is $\mathrm{W}_{\mathrm{l}}=2.5 \mathrm{~kg} \mathrm{~m}^{-2}$ at MAP $=650 \mathrm{~mm}$, as observed for example at Nylsvley, South Africa (Scholes and Walker, 1993; Holdo et al., 2013), assuming the ratio of aboveground biomass to total woody biomass to be 0.6 .

The grass growth coefficient $\left(\mathrm{g}_{\mathrm{g}}\right)$ and the woody plant growth coefficient $\left(\mathrm{g}_{\mathrm{w}}\right)$ are assumed to be constant along the rainfall gradient. Experimental measurement of $g_{g}$ and $g_{w}$ is lacked and thus I determined $\mathrm{g}_{\mathrm{g}}$ and $\mathrm{g}_{\mathrm{w}}$ assuming that the time for grasses and woody plants to reach carrying capacity is one growing season and 50 growing seasons, respectively. The values of $g_{g}$ and $g_{w}$ used in this study lead to a good agreement of vegetation composition (biomass of woody plants and grasses) with experimental observations (Sankaran et al., 2005; Bhattachan et al., 2012); sensitivity analysis indicates that a relatively small changes of $g_{g}$ and $g_{w}$ do not change the results. The ratio $\left(\alpha_{1} / \alpha_{2}\right)$ between root density of woody plants in the shallow and deep soil layer is taken to vary from 1 to 6 and is consistent with the assumption of exponential or linear root distributions reported by other authors (Lai and Katul et al., 2000; Schenk and Jackson, 2002; Kulmatiski and Beard, 2013b). Based on limited data reported by Bhattachan et al (2012), $\alpha_{1} / \alpha_{2}$ vary from 1.5 to 4 across the rainfall gradient (i.e., $200 \mathrm{~mm}<\mathrm{MAP}<700 \mathrm{~mm}$ ) in the Kalahari Transect. Following van Wilgen et al (2000), I express fire frequency ( $)$ as:

$$
\eta=\frac{\exp (q)}{1+\exp (q)} \quad \text { Eqn } 6
$$

where $\mathrm{q}=-2.47+2.35 \mathrm{G}_{\mathrm{l}}$ with $\mathrm{G}_{1}$ expressed in $\mathrm{kg} \mathrm{m}^{-2}$, assuming the ratio of aboveground biomass to total grass biomass to be 0.6 . Values of the model parameters are shown in 
Supplementary Table 4.2. Soil moisture dynamics are calculated with a time step of one hour while woody plant and grass dynamics with a time step of one day. The soil moisture is initialized at the wilting point of woody plants and grasses (Yu and D’Odorico, 2014a), while the landscape scale biomass of woody plants and grasses is initialized at $0.001 \mathrm{~kg} \mathrm{~m}^{-2}$. However, to investigate whether the dynamics exhibit alternative stable states, simulations are also run with woody plants and/or grasses initialized at a higher value of biomass (i.e., $90 \%$ of vegetation carrying capacity). I found that the dynamics may exhibit alternative stable states, consistent with previous models of savanna ecosystems (Walker et al., 1981; D'Odorico et al., 2006a; Staver et al., 2011a b). The simulations are run until the system attains a steady state (which typically occurs within 15-25 growing seasons) and I discard the initial transient. Landscapes with tree biomass $<0.05 \mathrm{~kg} \mathrm{~m}^{-2}$ are classified as grasslands, while above that limit they are considered as savannas. I allow for the existence of a low biomass of woody plants $(<\approx 0.05 \mathrm{~kg}$ $\mathrm{m}^{-2}$ ) in grasslands to investigate the effects of hydraulic redistribution (particularly hydraulic lift, HL) on the stability of savannas.

Studies of climate changes predict an increased occurrence of extreme rainfall regimes with increased rainfall intensity but reduced rainfall frequency (Knapp et al., 2002; Smith, 2011). To evaluate this effect, the sensitivity of this model is investigated with respect to changes in rainfall regime (i.e., increased rainfall intensity but consistent total amount of rainfall). Some studies indicate that root depth of woody plants (95\% of root biomass) vary from 0.6 to $1.2 \mathrm{~m}$ and tend to decrease with rainfall across the Kalahari rainfall gradient (Bhattachan et al., 2012; Gentine et al., 2012). For convenience of comparison and simplicity, root depth of grasses (i.e., $250 \mathrm{~mm}$ ) and woody plants (i.e., $700 \mathrm{~mm}$ ) is assumed to be constant across the Kalahari rainfall gradient. However, a sensitivity analysis of deeper rooted woody plants (i.e., $1000 \mathrm{~mm}$ ) was conducted for 
the case of dry savannas (MAP $<650 \mathrm{~mm}$ ). Additional runs of model are also preformed to assess the effects of soil texture. Soil texture (sandy loam) with lower hydraulic conductivity than sands will reduce the drainage from the shallow soil layer to the deep soil layer and thus reduce the magnitude of hydraulic lift (Yu and D'Odorico, 2014b). The parameters describing various soil characteristics used in this study are presented in Supplementary Table 4.3. A list of the abbreviations used to refer to the model variables is provided in Supplementary Table 4.1.

The initial validity of the model is evaluated by comparing the values of woody plant fractional cover $\left(f_{c}\right)$ to those reported by Sankaran et al (2005). As shown in Supplementary Figure 4.1, $\mathrm{f}_{\mathrm{c}}$ is less than 5\% when MAP $<220 \mathrm{~mm}$ and substantially increases with MAP (>220 $\mathrm{mm}$ ), in agreement with Sankaran et al (2005). At the landscape scale HL increases with MAP mainly because the fractional woody root cover, $\mathrm{f}_{\mathrm{r}}$ increase with MAP. These values of HL are within the range of those reported by other experimental studies in savannas (Meinzer et al., 2004; Scholz et al., 2010).

\subsection{Results}

\subsubsection{Dry savannas as affected by hydraulic lift}

Model results suggest that grasses outcompete woody plants in very dry environments (mean annual precipitation, MAP $<200 \mathrm{~mm})$, regardless of the ratio $\left(\alpha_{1} / \alpha_{2}\right)$ between woody root density in the shallow and deep soil layers (Figure 4.2a). While in relatively dry environments (MAP $<540-610 \mathrm{~mm}$ ) woody plants and grasses coexist regardless of the value of $\alpha_{1} / \alpha_{2}$, in semiarid environments with MAP $>540-610 \mathrm{~mm}$ (but still MAP $<650 \mathrm{~mm}$ ) woody plants even with relatively shallow roots (i.e., high values of $\alpha_{1} / \alpha_{2}$ ) outcompete grasses (Figure 4.2a). In fact, the precipitation is sufficient to sustain drainage to the deeper soil layer (Eqn 1c), where 
woody plants have exclusive access to water (Eqn 3c and Eqn 5b); these conditions favor woody

plants.
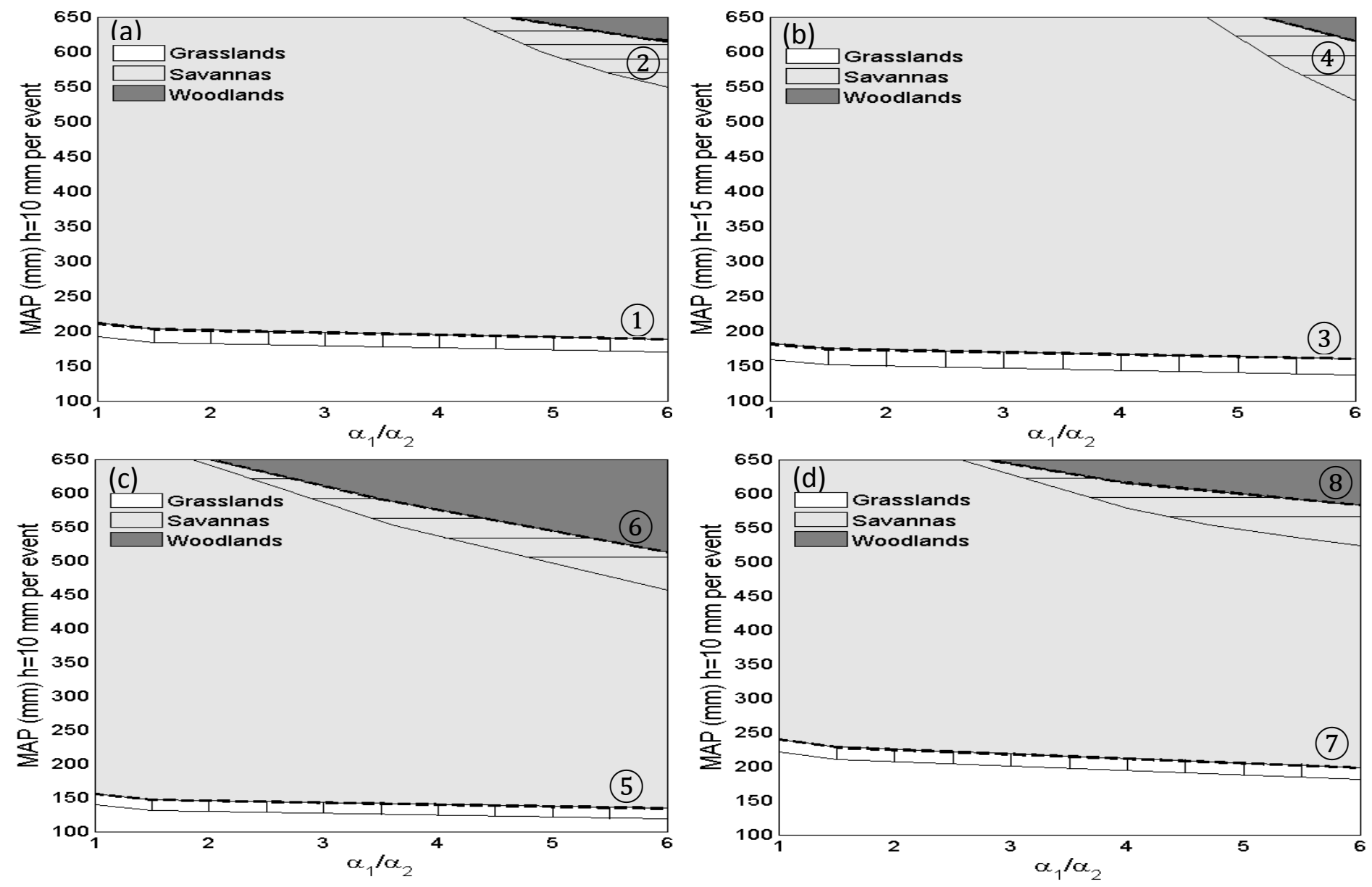

Figure 4-2: Grassland, savanna, and woodland domains as a function of mean annual precipitation (MAP) and the ratio $(\alpha 1 / \alpha 2)$ of woody plant root density in the shallow and the deep soil layer. Shading with horizontal lines (i.e., " $\equiv "$ indicates the band in the para parameter space in which the existence of savannas is induced by hydraulic lift (HL); in the absence of HL the landscape would be covered by woodland vegetation. Shading with vertical lines (i.e., "||l" indicates the zone of the parameter space where the existence of grasslands is due to hydraulic lift (HL); in the absence of HL savanna would instead exist. (a) Mean rainfall depth, $\mathrm{h}=10 \mathrm{~mm}$ per event; depth of the shallow soil layer, $\mathrm{Z}_{1}=0.25$ $\mathrm{m}$; depth of the deep soil layer, $\mathrm{Z}_{2}=0.45 \mathrm{~m}$; soil type: sand. Along line (1): $\mathrm{HL}=0.02-0.04 \mathrm{~mm} \mathrm{~d}^{-1}$. Along line (2): $\mathrm{HL}=0.21-0.23 \mathrm{~mm} \mathrm{~d}^{-1}$. (b) Mean rainfall depth, $\mathrm{h}=15 \mathrm{~mm}$ per event; depth of the shallow soil layer, $\mathrm{Z}_{1}=0.25 \mathrm{~m}$; depth of the deep soil layer, $\mathrm{Z}_{2}=0.45 \mathrm{~m}$; soil type: sand. Along line (3): $\mathrm{HL}=0.03-0.06 \mathrm{~mm} \mathrm{~d}^{-1}$. Along line (4): $\mathrm{HL}=0.36-0.37 \mathrm{~mm} \mathrm{~d}^{-1}$. (c) Mean rainfall depth, $\mathrm{h}=10$ $\mathrm{mm}$ per event; depth of the shallow soil layer, $\mathrm{Z}_{1}=0.25 \mathrm{~m}$; depth of the deep soil layer, $\mathrm{Z}_{2}=0.75 \mathrm{~m}$; soil type: sand. Along line (5): $\mathrm{HL}=0.01-0.03 \mathrm{~mm} \mathrm{~d}^{-1}$. Along line (6): $\mathrm{HL}=0.04-0.17 \mathrm{~mm} \mathrm{~d}^{-1}$. (d) Mean rainfall depth, $\mathrm{h}=10 \mathrm{~mm}$ per event; depth of the shallow soil layer, $\mathrm{Z}_{1}=0.25 \mathrm{~m}$; depth of the deep soil layer, $\mathrm{Z}_{2}=0.45 \mathrm{~m}$; soil type: sandy loam. Along line (7): $\mathrm{HL}=0.01-0.02 \mathrm{~mm} \mathrm{~d}^{-1}$. Along line (8): $\mathrm{HL}=0.06-0.11 \mathrm{~mm} \mathrm{~d}^{-1}$. 
The fraction $(\gamma)$ of woody plant biomass relying on the shallow soil layer increases with $\alpha_{1} / \alpha_{2}$ (Eqn 3b) (Supplementary Figure 4.2a, b, c); high values of $\gamma$ correspond to shallower rooted profiles of woody plants (Supplementary Figure 4.2b) and soil texture with a lower hydraulic conductivity (i.e., sandy loam) (Supplementary Figure 4.2c). With high values of $W_{l}$ and $\gamma$ woody plants strongly compete for water with grasses in the shallow soil layer (Eqn 3a), while maintaining exclusive access to the deep soil layer, thus providing the conditions that are conducive to the competitive exclusion of grasses. Note that the same of $\alpha_{1} / \alpha_{2}$ and thus $\gamma$ can be achieved in different environments, but environmental conditions such as a higher rainfall will increase biomass of woody plants $\left(W_{l}\right)$ and is thus conducive to the competitive exclusion of grasses. Overall, these results indicate that increase in the degrees of root separation between woody plants and grasses and reduction in rainfall promote coexistence by decreasing direct competitive effect of woody plants on grasses.

Model results also suggest that increase in mean rainfall depth $(h)$ (keeping a constant total annual rainfall) disfavors grasses (Eqn 3a) in dry environments (Figure 4.2) because of reduction of soil moisture in the shallow soil layer. Consequently, an increase in $\mathrm{h}$ reduces the range of mean annual precipitation (MAP) and $\left(\alpha_{1} / \alpha_{2}\right)$ ratios - between woody root density in the shallow and deep soil layers - corresponding to savanna conditions at the expense of woodlands (Figure 4.2a, b). Interestingly, I also find that in the more humid environments (still with MAP $<650 \mathrm{~mm}$ ) the advantage of woody plants at higher values of $h$ is reversed. For example, for MAP $=650 \mathrm{~mm}$, a value of $\alpha_{1} / \alpha_{2}=4.5$ allows for the coexistence of woody plants and grasses at $h=15 \mathrm{~mm}$ per event but leads to the competitive exclusion of grasses at $h=10 \mathrm{~mm}$ per event. This can be explained by a reduction of water losses due to drainage from the deeper soil layer $\left(D_{2}\right)$ and thus an increase in vegetation carrying capacity contributed by the deep soil 
layer $\left(V_{2}\right)$ with higher $\lambda$ especially when MAP is relatively high (Supplementary Figure 4.3a). Increase in root depth of woody plants favors woody plants and thus expands the range of mean annual precipitation (MAP) and $\left(\alpha_{1} / \alpha_{2}\right)$ ratios in which woodlands are stable (Figure 4.2a, c) because of reduction of $D_{2}$ (Supplementary Figure 4.3b). Soil texture (loamy sand) with a lower hydraulic conductivity than sand increases soil moisture in the shallow soil layer and thus favors grasses in dry environments (Figure 4.2a, d). Also interestingly, the advantage of grasses in reversed in the more humid environments (still with $\mathrm{MAP}<650 \mathrm{~mm}$ ) because of a low $D_{2}$ in sandy loam (Supplementary Figure 4.3c), which favors woody plants instead.

Hydraulic redistribution occurs predominately in the upward direction (hydraulic lift, HL) even in very dry environments because grasses maintain low soil water contents in the shallow soil layer (Yu and D'Odorico, 2014b). I can now examine the effects of HL on the interactions between woody plants and grasses and the stability of savannas. Note that in the simulations I allow a low woody plant biomass $\left(<\approx 0.05 \mathrm{~kg} \mathrm{~m}^{-2}\right)$ to exist in the grasslands. HL expands the range of parameters for which grasslands and savannas exist with respect to the case without $\mathrm{HL}$ (Figure 4.2). More specifically, if woody plants establish in the grassland, they tend to favor grasses by performing HL. The benefits grasses gain from HL tend to sustain grasslands and prevent the encroachment of woody plants. Likewise, when in more humid environments soil moisture in the deeper soil would promote woody plant dominance, HL can favor grass growth and thus sustain savannas. Higher values of HL and lower values of the fraction $(\gamma)$ of woody plant biomass competing with grasses favor grasses more. Thus the ability of HL to sustain savannas in areas that in the absence of HL would otherwise be woodlands (i.e., in the absence of $\mathrm{HL}$ ) is stronger when mean rainfall depth is higher, because these conditions enhance HL and reduce $\gamma$, as shown by the comparison between the case with mean rainfall depth, $h=15 \mathrm{~mm}$ 
per event (higher HL) and $h=10 \mathrm{~mm}$ per event (lower HL) (Figure 4.2a, b; Supplementary Figure 4.2a). Overall, these results also indicate that HL may be an alternative mechanism responsible for the coexistence of woody plants and grasses, as observed in the zones shaded with horizontal lines in Figures 2.

\subsubsection{Wet savannas as affected by hydraulic lift}

I also investigate the stability of relatively wet savannas induced by hydraulic lift (HL). I find that woody plants strongly compete with grasses in wet savannas, because of their preferential access to light and exclusive access to deep soil water. Thus, competitive exclusion of grasses occurs with high values of the fraction $(\gamma)$ of woody plant biomass competing with grasses (Eqn 3a) and with the low value of the light limitation coefficient $(l<1)($ Eqn $4 a)$. If I account for light limitation in grasses, in absence of fires woody plants and grasses can coexist only with $\mathrm{MAP}<\approx 1100-1300 \mathrm{~mm}$ and low ratios $\left(\alpha_{1} / \alpha_{2}\right)$ between the woody root densities in the shallow and deep soil (Figure 4.3a, b). For MAP $>\approx 1100-1300 \mathrm{~mm}$, the coexistence of woody plants and grasses can occur only in the presence of fires (Figure 4.3a, b). Hydraulic lift (HL) expands the range of parameters in which savannas can be stable at the expense of woodlands especially in regions with lower MAP (Figure 4.3a, b). 

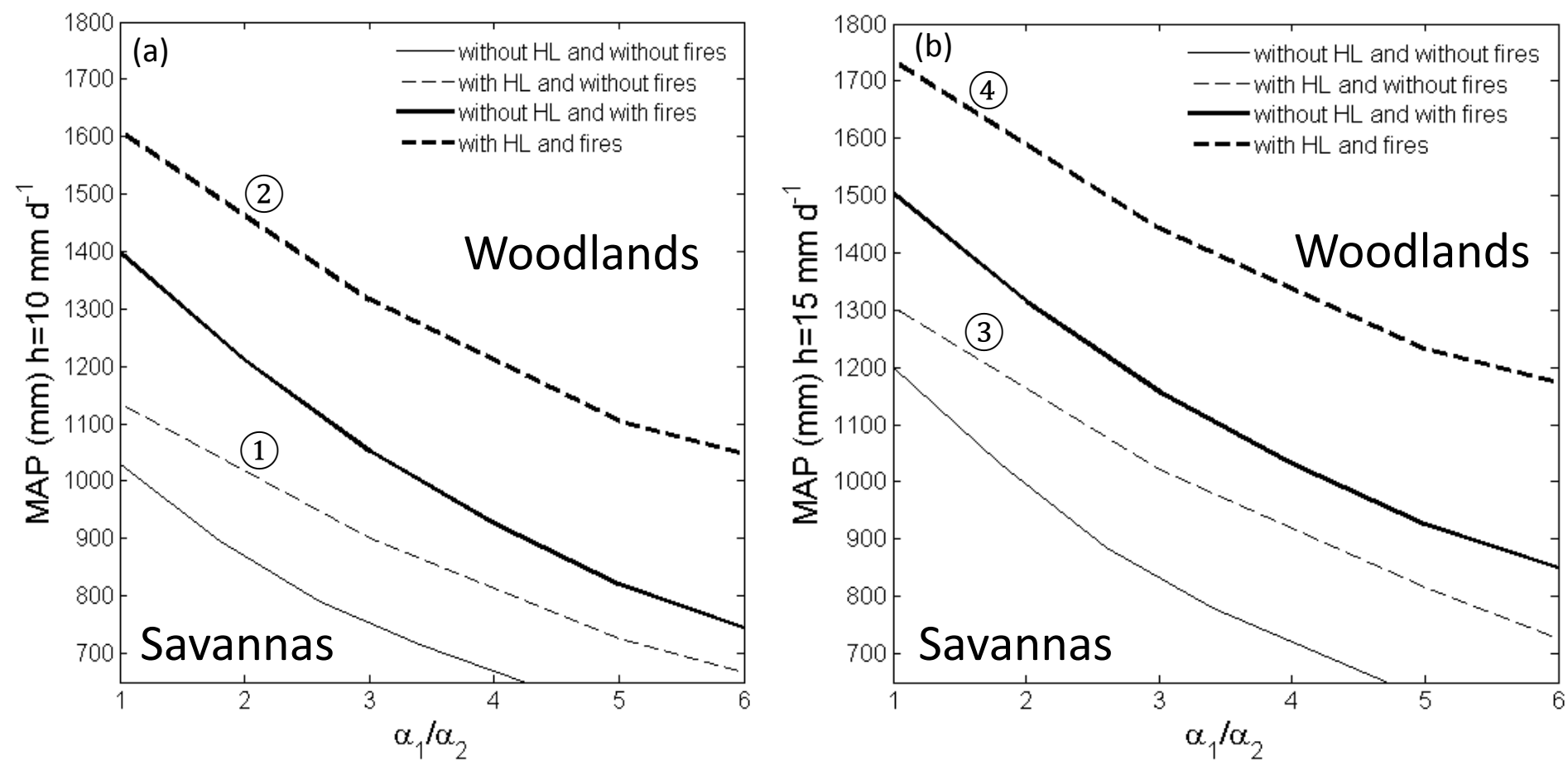

Figure 4-3: Transition between "wet" savanna and woodland vegetation as a function of mean annual precipitation (MAP) and the ratio $(\alpha 1 / \alpha 2)$ between woody root density in the shallow and the deep soil layer with and without hydraulic lift (HL) and fires. Points in the parameter space to the left of these lines correspond to savannas while points to the right correspond to woodlands. (a) Mean rainfall depth, $\mathrm{h}=10 \mathrm{~mm}$ per event; depth of the shallow soil layer, $\mathrm{Z}_{1}=0.25 \mathrm{~m}$; depth of the deep soil layer, $Z_{2}=0.45 \mathrm{~m}$; soil type: sand. Along line (1): $H L=0.13-0.22 \mathrm{~mm} \mathrm{~d}^{-1}$. Along line (2): $\mathrm{HL}=0.23-0.34 \mathrm{~mm} \mathrm{~d}^{-1}$. (b) Mean rainfall depth, $\mathrm{h}=15 \mathrm{~mm}$ per event; depth of the shallow soil layer, $\mathrm{Z}_{1}=0.25 \mathrm{~m}$; depth of the deep soil layer, $\mathrm{Z}_{2}=0.45 \mathrm{~m}$; soil type: sand. Along line (3): $\mathrm{HL}=0.24-0.35 \mathrm{~mm} \mathrm{~d}^{-1}$. Along line (4): $\mathrm{HL}=0.3-0.41 \mathrm{~mm} \mathrm{~d}^{-1}$. The following parameters were used: death rate of woody plants by fires, $\mathrm{k}=0.0002$.

\subsubsection{Tree-grass composition as affected by interannual rainfall variability}

\subsubsection{Impacts of interannual rainfall variability on tree-grass associations: satellite data}

Satellite data show a positive response of tree cover to increasing interannual rainfall variability in dry environments (i.e., mean annual precipitation, MAP $<900-1000 \mathrm{~mm}$ ) but a negative response of tree cover in wet environments (MAP > 900-1000 mm) (Figure 4.4a). The average proportion of burned areas (i.e., grass biomass) increases with increasing interannual rainfall 
variability in wet environments (i.e., MAP > 1000-1100 mm), in contrast to what happens in dry environments (i.e., MAP < 1000-1100 mm) (Figure 4.4b).
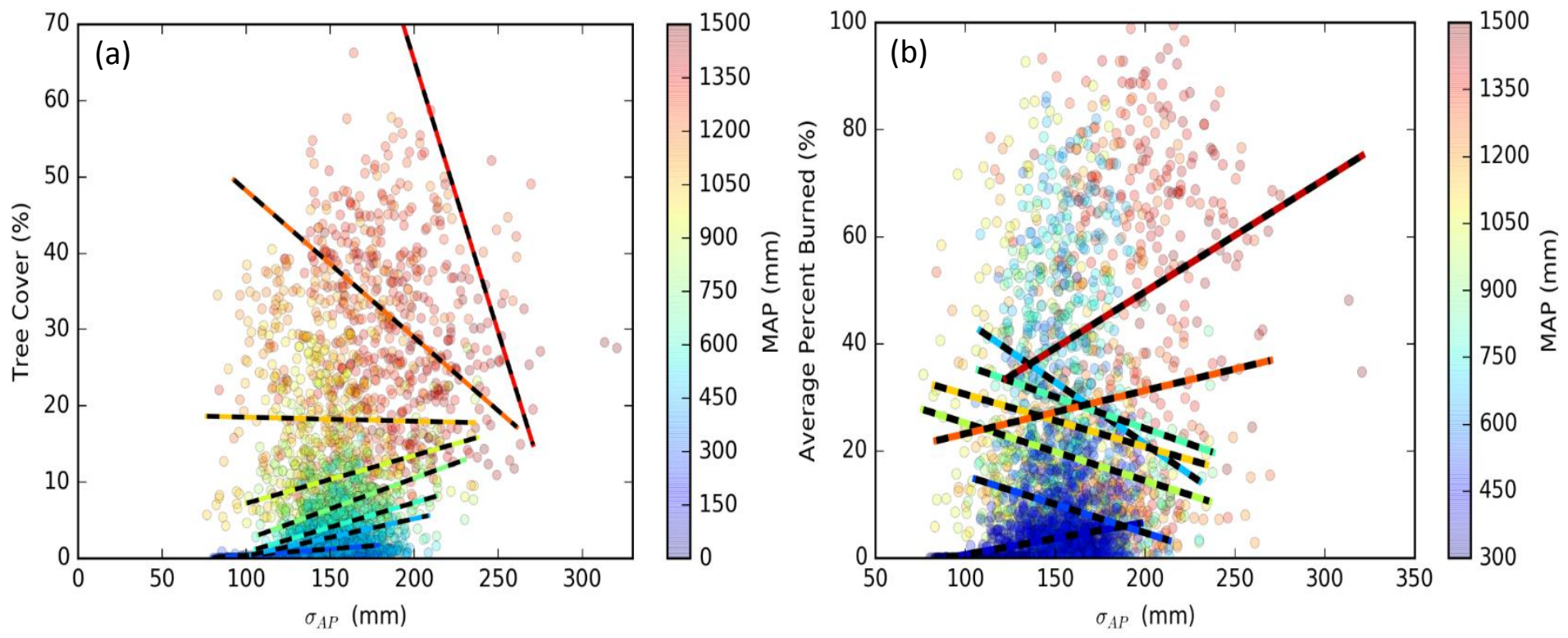

Figure 4-4: (a, b) MODIS-derived tree cover (a) and annual percentage burned (b) as a function of the standard deviation of annual precipitation $\left(\sigma_{\mathrm{AP}}, \mathrm{mm}\right)$ and mean annual rainfall (MAP, mm). MAP is binned into $150 \mathrm{~mm}$ bins and simple linear regression is used to highlight $\sigma_{\mathrm{AP}}$ related trends within these bins.

\subsubsection{Modeling impacts of interannual rainfall variability on tree-grass associations}

The modeling results show that in dry environments (i.e., $\lambda=0.2$ event $\mathrm{d}^{-1}, \mathrm{~h}=10 \mathrm{~mm}$ per event) tree-grass associations exhibit a substantial increase in tree biomass and cover, and a decrease in grass biomass with increasing interannual rainfall variability (Figure 4.5a), in agreement with my results based on satellite data (Figure 4.4). Grasses in tree-grass associations substantially decrease with increasing interannual rainfall variability because of increased competition from trees. In wet environments (i.e., $\lambda=0.4$ event $\mathrm{d}^{-1}, \mathrm{~h}=10 \mathrm{~mm}$ per event), grass biomass in tree- 
grass associations increases while tree cover in tree-grass associations decreases (Figure 4.5b), in agreement with satellite data (Figure 4.4).
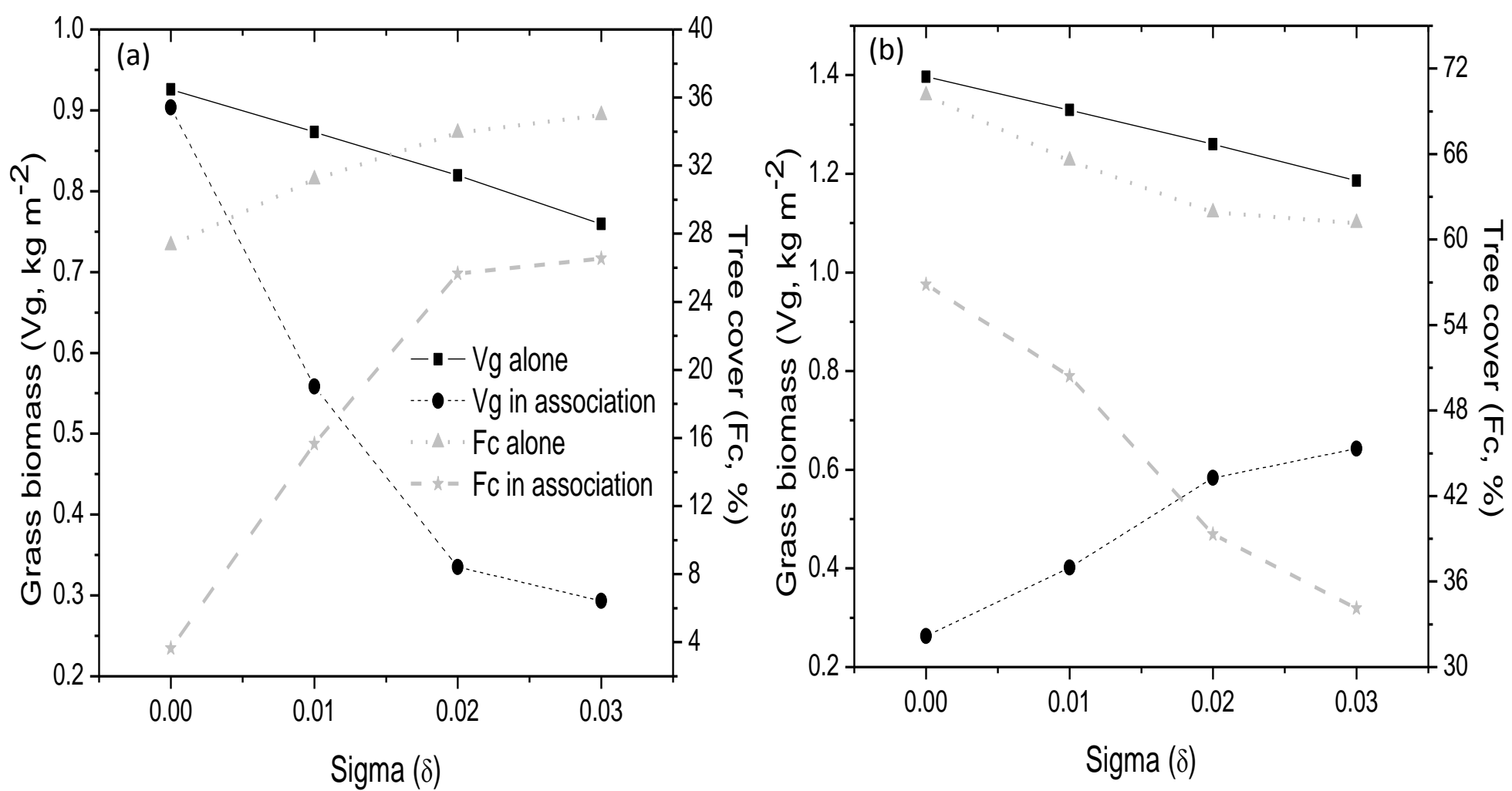

Figure 4-5: The modeled average grass biomass $(\mathrm{Vg})$ and tree cover $(\mathrm{Fc})$ in alone or associations for the case of sandy soil as affected by interannual rainfall variability (standard deviation of $\lambda$,

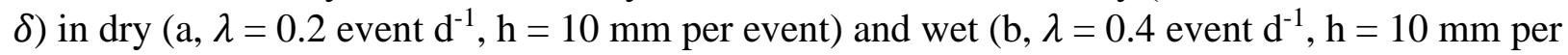
event) environments.

This pattern of grass in tree-grass associations, which is opposite to the results obtained in the case of grasses alone (Figure 4.5b), could be explained by the high growth rate of grasses and their ability to take advantage of increased pulses in shallow soil moisture with increasing interannual rainfall variability. The increase in grass biomass enhances the fire regime, thereby increasing fire-induced tree mortality and thus reducing competition from trees. 


\subsection{Discussion}

4.4.1 Hydraulic lift as a determinant of tree-grass coexistence on savannas

I investigate the factors affecting tree-grass coexistence in savannas. I focus on competitionbased mechanisms in dry savannas and disturbance-induced coexistence in wet savannas. The model developed in this study, however, accounts for both phenomena (i.e., competition and fires) at the same time, and thus provides an integrated framework for the study of resource- and disturbance-based mechanisms (Sankaran et al., 2004; Scheiter and Higgins 2007; Higgins et al., 2010). The role of hydraulic lift (HL) is accounted for by coupling the dynamics of woody plants and grasses with a soil water balance in the shallow and deep soil, and considering the effect of HL on soil moisture dynamics in both layers.

I revisit the Walter's two layer hypothesis by investigating the level of root separation necessary for the coexistence of woody plants and grasses. Early models in savanna ecology assume complete root separation as a requirement for the coexistence of woody plants and grasses (Walker and Noy-Meir, 1982; Eagleson and Segarra, 1985). Recent research indicates that woody plants and grasses can coexist even with substantial rooting overlap because of difference in resource uptake (Holdo et al., 2013; Ward et al., 2013). My results suggest that woody plants and grasses can coexist with substantial root overlapping $\left(\alpha_{1} / \alpha_{2}\right)$ in relatively dry environments (Figure 4.2a, b). Note that the same values $\alpha_{1} / \alpha_{2}$ can lead to different values of the fraction $(\gamma)$ of woody plant biomass relying on the shallow soil layer in different situations (Supplementary Figure 4.2a, b, c). Changes of rainfall regime may change the values of $\alpha_{1} / \alpha_{2}$ and $\alpha_{1} / \alpha_{2}$ was found to vary from 1.5 to 4 across the Kalahari rainfall gradient (Bhattachan et al., 2012). At the wetter end of what I have here defined as "dry savannas" (i.e., mean annual precipitation, MAP $<650 \mathrm{~mm}$ ) woody plants would displace grasses because of their competition 
with grasses (expressed by $\gamma \mathrm{W}_{\mathrm{l}}$ in Eqn 3a), which increases with the increase in mean annual precipitation (MAP) and root overlap (i.e, $\alpha_{1} / \alpha_{2}$ ). This is consistent with recent studies which suggest that restrictions on woody cover as a result of water limitation allow for the existence of grasses (Sankaran et al., 2005; Higgins et al., 2010). Increase in rainfall increases soil water in the deep soil and benefits woody plants more than grasses (Walker and Noy-Meir, 1982; van Langevelde et al., 2003), thus providing the conditions that are conducive to the competitive exclusion of grasses.

An increase in mean rainfall depth (h) - while keeping a constant MAP - reduces the soil moisture in the shallow soil layer, thereby disfavoring grass biomass and thus reducing the threshold values of MAP and root overlap (i.e., $\alpha_{1} / \alpha_{2}$ ) before grass exclusion occurs(Figure 4.2a, b). These results are consistent with the Walter's two layer hypothesis that the equilibrium ratio of woody plants and grasses depends on the vertical distribution of water in the soil profile with grass biomass increasing with the soil moisture in the shallow soil layer (Walker and NoyMeir, 1982; Sala et al., 1997; van Langevelde et al., 2003; Sankaran et al., 2004). The advantage of woody plants in rainfall regime with a higher $\mathrm{h}$ is reversed in the case of higher MAP (Figure 4.2a, b). These results are consistent with those by Kulmatiski and Beard (2013a), who found that that an increase in precipitation intensity (reduction in rainfall frequency) favors woody plant encroachment because of increased woody root depths. Increase in root depth of woody plants favors woody plants and thus allows for coexistence of woody plants and grasses in drier environments (i.e., MAP<200 mm) (Figure 4.2c), which is consistent with experimental observation (Bhattachan et al., 2012). Another factor affecting the vertical water distribution in the soil profile is soil texture. Soils with lower hydraulic conductivity are expected to be moister in the shallow soil layer and to provide conditions that favor grasses (Sala et al., 1989; Sankaran 
et al., 2004), as observed in Figure 4.2a and 2d. Likewise, the advantage of grasses in soil with lower hydraulic conductivity is reversed in the case of higher MAP (Figure 4.2a, d).

Hydraulic lift (HL) expands the range of grassland and savanna stability at the expense of woodlands (Figure 4.2). HL increases the soil moisture in the shallow soil layer by transporting soil water from the deep soil layer into the shallow soil (Dawson, 1993; Caldwell et al., 1998), thus promoting grass growth. I note that in this study grasses have access to water hydraulically lifted by woody plants, as shown by a number of other authors (Dawson 1993; Zou et al., 2005; Scott et al., 2008) and in contrast to other studies suggesting that the hydraulically lifted water may remain confined to microsites within the soil profile close to the roots that have performed hydraulic lift (Brooks et al., 2006). Recent studies indicate that grasses may take up lifted water through ecto- and endomycorrhizal networks (CMNs) (Egerton-Warburton et al., 2007; Warren et al., 2008). Past studies suggested that HL plays an important role in the facilitation of woody plants on grasses (Riginos et al., 2009; Moustakas et al., 2013; Dohn et al., 2013). My research developed a mechanistic model to evaluate this effect of HL. The inclusion in the model of the possible effects of groundwater (Hultine et al., 2004), is expected to further enhance HL, thereby benefiting grasses even more.

I also evaluated the interactions of woody plants and grasses in wet savannas. I find that without disturbances (fires) woody plants and grasses can coexist only at low levels of rainfall and root overlap (Figure 4.3). This results from the strong competition both for water and light (i.e., belowground for soil water and aboveground for light) that woody plants exert on grasses in wet savannas. This is in contrast to other studies which emphasized only the competitive effect for light (Sankaran et al., 2005; Higgins et al., 2010). Overall, my results support the disturbance-based mechanism of tree-grass coexistence in wet savannas (Sankaran et al., 2004), 
even without accounting for the stochastic nature of fire (D’Odorico et al., 2006a) and the variance existing in demographic growth of woody plants (Higgins 2000; Gardner 2006). Recent models generated similar results invoking intense root-shoot interactions (Scheiter and Higgins 2007; Higgins et al., 2010), whereby shoot biomass grows faster if the roots are not killed by fires. These root-shoot dynamics, however, are expected to occur in those species that can sprout after disturbances (such as fires) remove their shoots (Midgley 1996; Vest et al., 2004; Moreira et al., 2012).

This study suggests the existence of a mechanism of tree-grass coexistence induced by hydraulic lift (HL) both in dry (zone shaded with horizontal lines in Figure 4.2) and wet savannas (Figure 4.3). This mechanism works similarly to other buffer mechanisms (fires) that maintain the system away from the woodland state (Jeltsch et al., 2000). In fact, HL benefits grass biomass and thus prevents the system from transitioning to the woodland state. There is experimental evidence that understory plants (grasses and/shrubs) benefit from HL (Dawson 1993; Zou et al 2005). Nevertheless the plants engaged in HL and/or tree seedlings may still compete for lifted water (Ludwig et al., 2004; Quijano et al., 2012) and HL may occur in periods when the water requirements of understory plants are reduced (Meinzer et al., 2004), thus limiting the benefits of understory plants from HL.

Studies of hydraulic lift (HL) continue to engender scientific interest because of its wide occurrence in woody plants in different environments (see review by Neumann and Cardon, 2012). The magnitude of HL are substantial when compared with the amount of water utilized by the plants (Brook et al., 2006; Neumann and Cardon, 2012), thus significantly affecting plantplant interactions (Dawson 1993; Ludwig et al., 2004; Meinzer et al., 2004; Zou et al., 2005), hydrological and biogeochemical cycles (Jackson et al., 2000; Armas et al. 2011; Quijano et al., 
2013), and ecosystem productivity (Lee et al 2005; Scott et al., 2008; Domec et al., 2010). The ability of HL to induce/enhance tree-grass coexistence is a new paradigm in savanna ecology. In fact, the existing theories explain coexistence in savannas invoking niche separation (Walter 1971; Walker and Noy-Meir, 1982; Eagleson and Segarra, 1985), the role of disturbance such as fires (Sankaran et al., 2004; D’Odorico et al., 2006a), storage mechanisms (Higgins 2000; Gardner 2006), spatial interactions (Jeltsch et al. 1996, Rodriguez-IturbeI et al., 1999b; van Wijk and Rodriguez-IturbeI, 2002), or landscape heterogeneity (Kim and Eltahir, 2004), while the effect of HL on vegetation dynamics in savannas has remained poorly investigated. While these dynamics are determined by a complex set of interacting factors (Scholes and Archer, 1997; van Langevelde et al., 2003; Sankaran et al., 2004), the model developed here highlights the role of hydraulic lift as a possible determinant of savannas stability.

4.4.2 The effects of interannual rainfall variability on tree-grass composition along Kalahari rainfall gradient

Climate change studies predict an increase both in intra-annual and interannual rainfall variability (Easterling et al., 2000; Fischer et al., 2013; IPCC, 2013). While previous studies have investigated the ecological impacts of changes in intra-annual rainfall variability (Knapp et al., 2008; Kulmatiski and Beard, 2013a; Zeppel et al., 2014), the effects of interannual rainfall variability on vegetation composition and ecosystem processes is not well understood, mostly because of limitations in long-term observational data (Fatichi and Ivanov, 2014; Ng et al., 2015). This study uses satellite data and develops a new mechanistic model to investigate the response of tree/grass composition to increasing interannual rainfall variability along the Kalahari Transect in Southern Africa. 
Satellite data show that increasing interannual rainfall variability favors trees over grasses in dry environments (i.e., mean annual precipitation, MAP $<900-1000 \mathrm{~mm}$ ) and disfavors trees over grasses in wet environments (i.e., MAP > 900-1000 mm) (Figure 4.4). In contrast to this study, Holmgren et al (2013) used the satellite data to show a neutral response of tree cover to increasing rainfall variability in wet tropics in Africa, possibly because of soil texture effects and disturbance (i.e., grazing). Most of the other studies on this subject have used models as diagnostic tools to gain mechanistic understanding of ecosystem dynamics in response to increasing interannual rainfall fluctuations (Fernandez-Illescas and Rodriguez-Iturbe 2003; Tews et al., 2004; Tews et al., 2006; Williams and Albertson, 2006; Liedloff and Cook, 2007). This study develops a new mechanistic model to clarify the role of tree-grass competition for soil water resources and fire-induced disturbance as determinants of savanna response to changes in interannual rainfall variability.

Previous studies have stressed the role of competition in the response of vegetation composition to increasing interannual rainfall variability (Scanlon and Albertson, 2003; Illescas and Rodriguez-Iturbe, 2003), while the effect of fires has been ignored (but see Williams and Albertson, 2006). This new mechanistic model shows that in dry environments an increase in interannual rainfall variability leads to a reduction in grass competition and fire or an increase in competition from trees that cause a higher rate of reduction in grass biomass and a higher rate of increase in tree cover than in the case of tree or grass alone (Figure 4.5). The competitive advantage of trees results from deeper root systems than grasses, which allow trees to have exclusive access to increased deep soil water (on wet years) with increasing interannual rainfall variability. Extensive field studies in Africa have found deeper root systems in trees than grasses (Kulmatiski and Beard, 2013a b; Holdo and Nippert, 2015), although in some savannas grasses 
could have roots as deep as trees (Sankaran et al., 2004; Beckage et al., 2009). Consistent with this study, Gherardi and Sala (2015 a b) show that in a 6-y field experiment an increase in interannual rainfall variability shifts species composition in favor of deep rooted (i.e, trees) over shallow (i.e., grasses) rooted species.

Interestingly, my study shows that increases in interannual rainfall variability in wet environments shifts species composition favoring grasses over trees (Figure 4.4; Figure 4.5). This is a novel finding because other studies in grasslands (Hsu et al., 2012) have shown that grasses alone have a negative response to increasing interannual rainfall variability in wet environments. Grasses typically have a high growth rate especially in wet environments (Teuling et al., 2010; Collins et al., 2012; Xu et al., 2015), consistent with the concept of the world-wide 'fast - slow' plant economics spectrum (Reich, 2014); thus, grasses could quickly take advantage of the window of opportunity existing in years with above average precipitation. The high growth rate in grasses increases fire frequency and fire-induced tree mortality (Bond et al., 2008; Ratajczak et al., 2014), thereby leading to a reduction in tree competition with grasses for soil water, which further favors grass biomass. These results are in agreement with a general theory of invisibility in plant communities under fluctuating resources (Davis et al., 2000). Based on field studies (Davis and Melissa, 2001; Corbin and D'Antonio, 2004), this theory holds that fluctuations in resource availability provide windows of opportunity in resource enrichment, whereby species with a high growth rate could quickly take up resources, change the disturbance regime, and then invade or dominate the landscape. Consistent with these studies, my study shows that the way tree-grass composition responds to increased interannual variation in precipitation would depend on key traits of trees and grasses (i.e., growth rate and root depth) 
that determine their ability to take advantage of the windows of opportunity offered by periods with higher soil moisture.

The encroachment of woody plants into grasslands has been widely documented in arid and semiarid environments in many regions of the world including southern Africa (Moleele et al., 2002). The mechanisms typically invoked to explain this phenomenon involve exogenic drivers including overgrazing, fire suppression, increase in $\mathrm{CO}_{2}$ concentration, and long-term global changes in rainfall or temperature and endogenic positive feedbacks (D'Odorico et al., 2012; Yu and D’Odorico, 2014a). This study shows that the increase in tree dominance in dry environments may also result from an increase in interannual rainfall variability.

A number of studies have invoked fire-vegetation feedbacks to explain the existence of savannas in a wide range of rainfall conditions in southern Africa (Staver et al., $2011 \mathrm{a}$ b). The increase in grass biomass and thus fire frequency in wet environments found in this study indicates that interannual rainfall fluctuations may expand the range of environmental conditions in which savannas are stable. This idea is in agreement with the emerging view that the interplay between tree/grass growth rate and fires regimes govern savanna-forest transitions (Hoffmann et al., 2012; Murphy et al., 2012).

The model developed in this study expressed vegetation capacity as a function of instantaneous soil moisture and thus allows for a process-based analysis of impacts of increasing interannual rainfall variability and/or soil texture on tree-grass composition. We also noted that an alternative modeling approach relating vegetation capacity to mean growing seasonal rainfall and root depth by trees and grasses did not change the general pattern found in this study. Moreover, we notice that this model does not account for other factors, including rainfall seasonality (Vico et al., 2015) and plant life histories (i.e., annuals or perennials; evergreen or 
deciduous) (Kos et al., 2012), which play an important role in determining plant community composition in savannas. In fact, an increase in winter rainfall increases deep soil water and thus favors trees (deep rooted plants) over grasses (shallow rooted plants) (Brown et al., 1997; Germino and Reinhardt, 2014). The high inter-annual variability of rainy season duration favors deciduous trees over evergreen trees, which may affect the competition with grasses (Vico et al., 2015). The legacy effect of trees and grasses attributed to water and/or carbohydrate storage, available seeds/meristems, and/or nutrient availability from litter decomposition in response to rainfall fluctuations are also expected to be affected by plant life histories (Sherry et al., 2008; Sala et al., 2012; Anderegg et al., 2015).

In summary, this study found that hydraulic lift is an important mechanism responsible for the coexistence of woody plants and grasses in savannas. This mechanism works because hydraulic lift increases soil moisture in the shallow soil where grasses could get access to and are thus beneficial. With the benefits gained by grasses, hydraulic lift expands (at the expense of woodlands) the range of environmental conditions in which savannas are stable. This study also used satellite data and a mechanistic model to show that deep rooted trees are favored over shallow rooted grasses in drier environments in response to increasing interannual rainfall fluctuations (i.e., mean annual rainfall, MAP<900-1000 mm), in contrast to wetter environments (i.e., MAP>900-1000 mm). We suggest that the relative magnitude of the growth rates and root depths of grasses and trees greatly affects the response of tree-grass composition in response to increasing interannual rainfall variability. 


\section{Plants with Crassulacean Acid Metabolism outcompete grasses under carbon dioxide enrichment and drought}

This chapter is adapted from Yu KL, D’Odorico P, Collins S, Carr D, Porporato A, et al., Plants with Crassulacean Acid Metabolism outcompete grasses under carbon dioxide enrichment and drought (under review).

\section{Abstract}

Plants with Crassulacean Acid Metabolism (CAM) are increasing in abundance in drylands worldwide but the underlying drivers remain unknown. In particular, concurrent elevated carbon dioxide $\left(\mathrm{CO}_{2}\right)$ concentrations and water stress - the important drivers which have been found to greatly affect $\mathrm{C}_{3}$ or $\mathrm{C}_{4}$ plants - may allow CAM plants to outcompete co-occurring $\mathrm{C}_{4}$ plants, but this has not been tested. Here, I report on experiments to investigate the competitive relationships between seedlings of Cylindropuntia imbricata (CAM) and Bouteloua eriopoda $\left(\mathrm{C}_{4}\right.$ grass $)$, which coexist in semiarid ecosystems across the Southwestern United States. Using growth chambers I altered $\mathrm{CO}_{2}$ and water conditions, this study shows that $C$. imbricata positively responded to $\mathrm{CO}_{2}$ enrichment under drought conditions, while $B$. eriopoda died from water stress. Conversely, in well-watered conditions B. eriopoda had a strong competitive advantage over $C$. imbricata, thereby reducing the photosynthetic rate and biomass (per individual) of $C$. imbricata grown with B. eriopoda, when compared to C. imbricata alone. Although $\mathrm{CO}_{2}$ enrichment increased the photosynthesis and biomass of both C. imbricata and B. eriopoda when grown alone, in mixtures $\mathrm{CO}_{2}$ enrichment benefited $C$. imbricata more than B. eriopoda and thus indirectly disfavored B. eriopoda under drought conditions. A meta-analysis of growth experiments for plants across multiple families shows a positive response of CAM photosynthesis and productivity to $\mathrm{CO}_{2}$ enrichment. My results suggest that drought and elevated 
$\mathrm{CO}_{2}$ concentrations projected with climate change favor CAM plants with likely continuing increases of these plants in semiarid ecosystems.

\subsection{Introduction}

Over the last century, human activities associated with fossil fuel burning and land use change have dramatically increased the concentrations of $\mathrm{CO}_{2}$ and other trace gases in the atmosphere, a trend that is expected to continue in the decades to come (e.g., Vitousek et al., 1997; IPCC 2014). At the same time, global climate models predict increased precipitation variability along with more frequent extreme rainfall events, and increased risk of prolonged drought, especially in dryland regions (Smith, 2011; IPCC, 2014). These changes in environmental conditions combined with human activities have led to significant changes in vegetation cover and plant community composition (Poorter and Navas, 2003; D’Odorico et al., 2012; Anderegg et al., 2013), with important impacts on ecohydrological and geochemical processes, regional climate and the provision of ecosystem services such as livestock grazing, sheltering of the soil surface, and carbon sequestration (Anderegg et al., 2013; Reichstein et al., 2013).

In particular, previous studies in the Southwestern United States have focused on the transition from $\mathrm{C}_{4}$ grasslands to $\mathrm{C}_{3}$ shrublands (e.g., D'Odorico et al., 2012) or widespread tree mortality under global environmental change (e.g., Anderegg et al., 2013). An empirical evaluation of the response of plants with Crassulacean Acid Metabolism (CAM) and their competitive relationships with other functional groups (i.e., $\mathrm{C}_{3}$ and $\mathrm{C}_{4}$ plants) to $\mathrm{CO}_{2}$ enrichment and changing climate, however, is still missing (Poorter and Navas, 2003). The phenomenon of increased dominance of CAM plants could provide new opportunities for the use of marginal lands for human adaptation to climate change by promoting bioenergy production and carbon 
sequestration (Borland et al., 2009). Some studies suggest that changes in climate (i.e., temperature or rainfall) or increasing atmospheric $\mathrm{CO}_{2}$ concentrations could be major drivers of CAM expansion in drylands (Drennan and Nobel, 2000; Borland et al., 2009; Reyes-García and Andrade, 2009), but this has yet to be tested experimentally.

CAM plants feature water storage in their tissues, nocturnal $\mathrm{CO}_{2}$ uptake, photosynthetic plasticity, and high water use efficiency (Drennan and Nobel, 2000; Lüttge, 2004; Borland et al., 2009), which could explain why their abundance appears to be increasing in many drylands worldwide. This phenomenon, however, also depends on the competitive relationship of CAM plants with other functional groups (i.e., $\mathrm{C}_{3}$ and $\mathrm{C}_{4}$ plants) (Poorter and Navas, 2003; Yu and D'Odorico, 2015), an aspect of CAM plant sensitivity to changes in climate and atmospheric $\mathrm{CO}_{2}$ concentrations that has remained largely understudied. Indeed, global environmental change (i.e., drought intensification) could disfavor other functional groups, thus indirectly increasing the competitive advantage of CAM plants. Conversely, $\mathrm{CO}_{2}$ enrichment may favor CAM plants more than $\mathrm{C}_{4}$ species, thus leading to the indirect disadvantage of $\mathrm{C}_{4}$ plants.

Here, we are the first to investigate the potential shift in the competitive relationships between CAM plants and $\mathrm{C}_{4}$ plants under global environmental change. To this end, we developed a set of growth chamber experiments in which two species, Cylindropuntia imbricata (CAM) and Bouteloua eriopoda ( $\mathrm{C}_{4}$ grass) co-occurring in desert grasslands in the southwestern United States and northern Mexico (i.e., Chihuahuan Desert) were subjected to two levels of $\mathrm{CO}_{2}$ concentrations under drought and well-watered conditions. Field observations in native and protected desert grasslands indicate that $C$. imbricata has been increasing in abundance in desert grasslands currently dominated by B. eriopoda (Supplementary Figure 5.1). The underlying mechanisms, however, remain unclear. 


\subsection{Materials and methods}

\subsubsection{Germination of seeds and growth of seedlings}

Seeds of the CAM plant, Cylindropuntia imbricata and the $\mathrm{C}_{4}$ grass, Bouteloua eriopoda, were germinated on 5 July 2015 in plastic trays covered with 1-2 mm of mineral soil in the greenhouse facility at the University of Virginia. Seed scarification through soaking in water for 10 hours was conducted for $C$. imbricata to improve germination rate. Seedlings (of similar size) of $C$. imbricata and B. eriopoda were transplanted into plastic pots $(13 \mathrm{~cm}$ in diameter and $11.5 \mathrm{~cm}$ in height) on 20 August 2015. C. imbricata and B. eriopoda were planted in either monoculture (4 individuals) or mixture ( 2 individuals of $C$. imbricata and 2 individuals of B. eriopoda). On 9 September 2015 seedlings $(6-7 \mathrm{~cm}$ in height for $C$. imbricata and $35-38 \mathrm{~cm}$ in height for $B$. eriopoda) were transported to Duke University.

\subsubsection{Experimental design and growth chambers}

Pots of 10 replicates containing seedlings of $C$. imbricata and B. eriopoda in both monoculture and mixture were subjected to two levels of $\mathrm{CO}_{2}$ concentrations and two water treatments: 400 ppm and 800 ppm under drought and well-watered conditions, using $\mathrm{CO}_{2}$ chambers at the Duke University Phytotron facility. Watering intensity and frequency were based on the 30-year mean values of growing season rainfall appropriate for the Sevilleta National Wildlife Refuge (Petrie et al., 2014), an arid grassland where C. imbricata and B. eriopoda co-exist (Miller et al., 2009). To investigate the effects of extreme drought on plant growth under future climate (Easterling et al., 2000; Smith, 2011; IPCC, 2014), for the first 63 days (the first stage until Novenber 13, 2015) plants were watered every nine days with an intensity of $4.5 \mathrm{~mm}$ per event in drought conditions (low water treatment, LW). To investigate the ability of plants to recover from 
extreme drought events (Volaire et al., 1998; Rivero et al., 2007), starting on day 64 all plants (from the LW treatment) were watered every three days with an intensity of $4.5 \mathrm{~mm}$ per event for 39 more days (the second stage until December 23, 2015). By comparison, in well-watered conditions (high water treatment, HW) plants were watered every three days with an intensity of $4.5 \mathrm{~mm}$ per event over 102 days. Nitrogen (1/2 strength Hoagland) was applied once every nine days to all the pots to support plant growth. Plants in chambers were subjected to controlled light conditions (i.e., 12 hours of light with photosynthetically photon flux density (PPFD) of 600-700 umol $\mathrm{m}^{-2} \mathrm{~s}^{-1}$ from 7 am to $7 \mathrm{pm}$ Eastern Daylight saving Time, EDT) and temperature (i.e., $25^{\circ} \mathrm{C}$ during the day and $20^{\circ} \mathrm{C}$ during the night) conditions. The experiment was a split-plot design in which $6 \mathrm{CO}_{2}$ chambers were used for treatments of two $\mathrm{CO}_{2}$ concentration gradients (each $\mathrm{CO}_{2}$ level had three replicates) and in each $\mathrm{CO}_{2}$ chamber seedlings were randomly assigned to treatments of drought and well-watered conditions (each treatment had 10 replicates). The growth chambers were manufactured by Environmental Growth Chambers (EGC), Chagrin Falls, Ohio 44022.

\subsubsection{Soil and soil moisture}

A mixture of Canadian sphagnum peat moss and calcined clay (6:3) was used as soil (Kieft, 1998). Soil moisture content was calculated as the ratio of the mass of water to dry soil and was determined gravimetrically by drying samples at $60{ }^{\circ} \mathrm{C}$ for $72 \mathrm{~h}$.

\subsubsection{Gas exchange}

Gas exchange between plant and surrounding air was measured at the end of the second stage of the experiment. The shoots of the CAM plants $C$. imbricata are stems with cylindrical shape (Supplementary Figure 5.2). To measure gas exchange of $C$. imbricata, a customized Plexiglas cylindrical chamber $(7 \mathrm{~cm}$ in diameter and $12.5 \mathrm{~cm}$ in height) closed on one end and with a hole 
(1.5 cm in diameter) on the other was built. The chamber (model Li-6400-19 Custom Chamber

Kit, LiCor Inc., Lincoln, NB) was then interfaced to a portable photosynthesis unit (model LI6400XT, LiCor Inc.). Measurements of gas exchange were conducted for each individual of $C$. imbricata using LI-6400XT unit with the leaf area (a) of $2 \times 3 \mathrm{~cm}^{2}$ in a closed system mode. The net photosynthetic rate $\left(A\right.$, umol $\left.\mathrm{m}^{-2} \mathrm{~s}^{-1}\right)$ of $C$. imbricata was determined as $\mathrm{A}=\mathrm{A}_{\mathrm{r}} \times \mathrm{a} / \mathrm{S}$, where $\mathrm{A}_{\mathrm{r}}$ is the recorded value of the net photosynthetic rate by LI-6400XT and S is the total photosynthetic surface area $\left(\mathrm{cm}^{2}\right)$ of $C$. imbricata. After harvesting, the surface area of each plant stem was calculated as follows: depending on the shape of $C$. imbricata, each $C$. imbricata stem was cut into 3-4 sections, each of which represents a more regular cylinder shape and has the surface area of $\pi \times d \times h(d$ is the stem diameter and $h$ is the lengths of each stem segment $)$; $\mathrm{S}$ is the sum of the surface areas of all sections. Diameter and height were measured by a caliper with a resolution $0.001 \mathrm{~mm}$. Diurnal change of gas exchange for $C$. imbricata was measured at a constant leaf temperature of $20^{\circ} \mathrm{C}$ using three replicates in each treatment in each chamber. The $\mathrm{CO}_{2}$ mixing ratio was set to $400 \mathrm{ppm}$ at ambient $\mathrm{CO}_{2}$ conditions while the $\mathrm{CO}_{2}$ mixing ratio was set to $800 \mathrm{ppm}$ at high $\mathrm{CO}_{2}$ conditions. The light intensity was the same as in the environment inside each chamber.

\subsubsection{Titratable acidity}

Titratable acidity was measured at the end of the second stage of the experiment. Stems of $C$. imbricata were sampled at $3 \mathrm{~h}$ intervals and stored at $-20{ }^{\circ} \mathrm{C}$. Each stem was cut into 3-4 discs; one disc with a regular cylindrical shape was selected to measure titratable acidity. Diurnal change in titratable acidity $\left(\mathrm{mmol} \mathrm{m}^{-2}\right)$ was measured using the acid base titration method (Caemmerer and Griffiths, 2009), in which freshly made $10 \mathrm{mM}$ sodium hydroxide $(\mathrm{NaOH})$ was 
added into a $C$. imbricata solution with $20 \mathrm{uL}$ of a $1 / 5$ dilution of phenolphthalein as indicator after boiling for $10 \mathrm{~min}$.

\subsubsection{Plant biomass and productivity}

Plants were harvested on Nov 13, (the end of the first stage) and Dec 23, 2015 (the end of the second stage), respectively for measuring plant biomass and productivity. When grown in mixture, $C$. imbricata and B. eriopoda were separated; a small amount of root fragments of $C$. imbricata or B. eriopoda remained in the soil after plant separation and were identified based on color, diameter and shape. Shoots (leaves and/or stems) and roots were separated. Roots were washed free of soil through 0.1-mm mesh sieves. Fresh shoots were weighted on a scale; both shoots and roots were dried at $60^{\circ} \mathrm{C}$ for $72 \mathrm{~h}$ and weighted. Total biomass and shoot-to-root biomass ratios were calculated. Relative aboveground plant water content was determined as (fresh weight-dry weight) / fresh weight, \%).

\subsubsection{Carbon isotope analysis}

Carbon dioxide was sampled in each chamber using gas-tight vials. The carbon isotope composition of $\mathrm{CO}_{2}$ gas samples $\left(\delta^{13} \mathrm{C}_{\mathrm{g}}\right)$ was analyzed at Indiana University Purdue University Indianapolis on a stable isotope ratio mass spectrometer (IRMS) (Model Delta V Plus, Thermo

Fisher Scientific, Waltham, MA) connected under continuous flow to a headspace gas analyzer (Thermo, GasBenchII). Aboveground plant samples were dried at $60^{\circ} \mathrm{C}$ for $72 \mathrm{~h}$ and then ground and homogenized for isotope and elemental analysis. Aboveground plant samples were combusted in an elemental analyzer (Costech Analytical, ECS 4010) and the carbon isotope composition of aboveground plant samples $\left(\delta^{13} C_{p}\right)$ was analyzed under continuous flow on the 
same IRMS. Analytical precision for replicate analysis of standard reference materials was \pm $0.1 \%$. Aboveground plant carbon isotope discrimination $(\Delta)$ was calculated as, $\Delta=\delta^{13} C_{g}-\delta^{13} C_{p}$.

\subsubsection{Meta-analysis}

A meta-analysis was conducted to examine the response of CAM photosynthesis and productivity to $\mathrm{CO}_{2}$ enrichment reported by previous studies. We performed multiple Google Scholar and Web of Science searches using keywords of crassulacean acid metabolism (or CAM, cactus, succulent), $\mathrm{CO}_{2}$, photosynthesis, and productivity. To be included in the meta-analysis, the paper needed to report a long term ( $\geq 2$ months) response of photosynthesis, biomass (productivity), or growth rate to $\mathrm{CO}_{2}$ enrichment which spans a range of $650 \mathrm{ppm}$ to $1000 \mathrm{ppm}$ in optimal environments (i.e., adequate light and nutrient availability). We found that the published studies were scarce and identified 21 studies spanning 19 CAM species among 5 families across the Southwestern United States, Israel, Panama, Singapore, and China. For those studies which did not report standard deviation and/or standard error, standard deviation and/or standard error were determined as the mean of all CAM species in each family.

\subsubsection{Data analysis}

The effects of $\mathrm{CO}_{2}$, water treatment, species, competition and time as well as their interactions on total biomass, the rate of total biomass change, shoot to root biomass ratio, aboveground plant carbon isotope discrimination, and relative aboveground plant water content were analyzed by a five-way ANOVA with each chamber as a whole-plot random factor. The effects of $\mathrm{CO}_{2}$, water, competition and time as well as their interactions on soil moisture were analyzed by a four-way ANOVA with each chamber as a whole-plot random factor. Data of total biomass was natural log transformed prior to ANOVA. In general, the most interesting effects were found in multiway interactions. To explore these interactions, I constructed pairwise orthogonal contrasts to detect 
differences between individual pairs of means. All these statistics were performed in SAS 9.4.

The meta-analysis was conducted using "metafor" package in R.

\subsection{Results}

$\mathrm{CO}_{2}$ enrichment significantly increased the biomass of $C$. imbricata and B. eriopoda alone in both drought and well-watered conditions (all $\mathrm{P} \leq 0.0184$ for $C$. imbricata, all $\mathrm{P} \leq 0.0372$ for $B$. eriopoda, Figure 5.1).
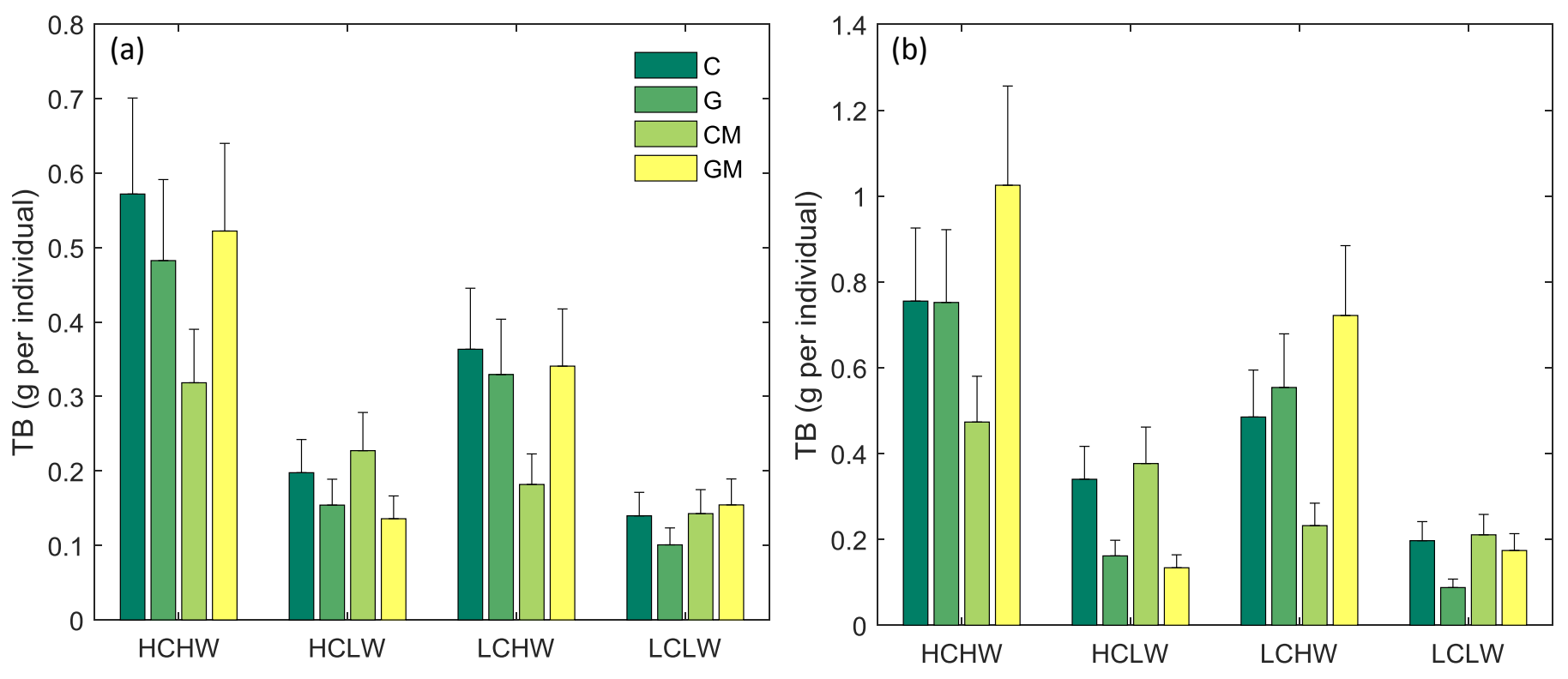

Figure 5-1: Total biomass (TB, g) of Cylindropuntia imbricata (CAM) and Bouteloua eriopoda $\left(\mathrm{C}_{4}\right.$ grass) in CAM alone (C), grass alone (G), mixture (CM, GM) in the first stage (a, November 13th 2015) and the second stage (b, December 23th 2015) in each treatment. HCHW refers to high $\mathrm{CO}_{2}$ and well-watered conditions; $\mathrm{HCLW}$ refers to high $\mathrm{CO}_{2}$ and drought conditions; $\mathrm{LCHW}$ refers to low $\mathrm{CO}_{2}$ and well-watered conditions; LCLW refers to low $\mathrm{CO}_{2}$ and drought conditions. The error bars represent $95 \%$ confidence intervals.

$\mathrm{CO}_{2}$ enrichment increased $\mathrm{CO}_{2}$ uptake by $C$. imbricata both at night through $\mathrm{C}_{4}$ photosynthesis ( $8 \mathrm{pm}$ to $8 \mathrm{am})$ and during the late afternoon with $\mathrm{C}_{3}$ photosynthesis $(\sim 4-7 \mathrm{pm})$ (Figure 5.2a, c). The increase in $\mathrm{CO}_{2}$ uptake at night in response to $\mathrm{CO}_{2}$ enrichment increased the accumulation of malic acid in the vacuole and thus titratable acidity (Figure 5.2b, d). 

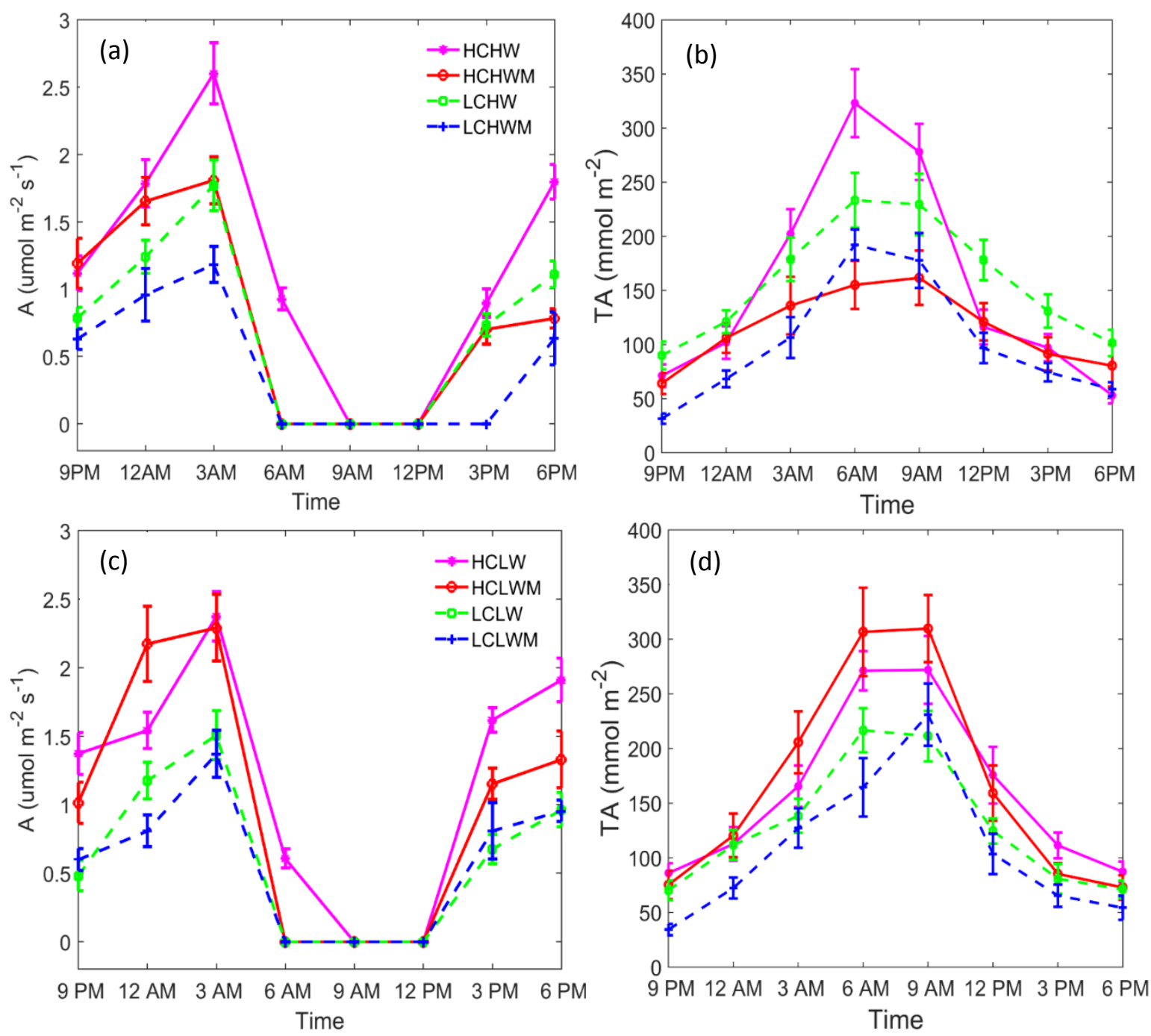

Figure 5-2: Diurnial change of rates of carbon dioxide uptake (A, umol m $\left.\mathrm{m}^{-2} \mathrm{~s}^{-1}\right)$ and titratable acidity $\left(\mathrm{mmol} \mathrm{\textrm {m } ^ { - 2 } )}\right.$ by Cylindropuntia imbricata in well-watered $(\mathrm{a}, \mathrm{b})$ and drought $(\mathrm{c}, \mathrm{d})$ conditions. $C$. imbricata performs $\mathrm{C}_{4}$ photosynthesis at night and $\mathrm{C}_{3}$ photosynthesis in the late afternoon, as shown by tritiable acidicity measurements. Values are mean \pm standard deviation (n $=9$ ).

The response of mixed CAM- $\mathrm{C}_{4}$ communities to $\mathrm{CO}_{2}$ enrichment depended crucially on water conditions $\left(\mathrm{CO}_{2} \times\right.$ water $\times$ competition $\mathrm{P}<0.0001$, Supplementary Table 1$)$. While $\mathrm{CO}_{2}$ enrichment significantly increased the biomass of both B. eriopoda and C. imbricata in mixture in well-watered conditions in both stages (both $\mathrm{P} \leq 0.0169$ for $B$. eriopoda, both $\mathrm{P} \leq 0.0002$ for C. imbricata), $\mathrm{CO}_{2}$ enrichment only favored $C$. imbricata in mixture in drought conditions $(\mathrm{P} \leq$ 
0.0016 for $C$. imbricata, $\mathrm{P} \geq 0.0731$ for B. eriopoda, Figure 5.1). Moreover, in ambient $\mathrm{CO}_{2}$ and drought conditions (LCLW) the biomass of B. eriopoda in mixture was significantly higher than that in grass alone (for both stages $\mathrm{P}<0.0001$, Figure 5.1), in contrast to the case of HCLW (for both stages $\mathrm{P} \geq 0.0632$, Figure 5.1). Overall, these results suggest that under drought conditions C. imbricata responded more positively to $\mathrm{CO}_{2}$ enrichment than B. eriopoda $\left(\mathrm{CO}_{2} \times\right.$ species $\mathrm{P}<$ 0.0001, Supplementary Table 1) and thus $\mathrm{CO}_{2}$ enrichment indirectly disfavored the $\mathrm{C}_{4}$ grass in mixture.

The increase in water availability significantly increased the biomass of both $C$. imbricata and B. eriopoda when grown alone and in mixture, except for C. imbricata in mixture under ambient $\mathrm{CO}_{2}$ conditions in the second stage $(\mathrm{LCS})(\mathrm{P}=0.3381$ for $\mathrm{LCS}$; all $\mathrm{P} \leq 0.0242$ for other conditions, Figure 5.1). C. imbricata had a higher relative aboveground plant water content than B. eriopoda (all $\mathrm{P}<0.0001$, Supplementary Figure 5.3) and its growth, which was limited by drought in the first stage, recovered quickly in the second stage of well-watered conditions (Supplementary Figure 5.4). By comparison, all individuals of B. eriopoda appeared to die in the first stage under drought conditions (Supplementary Table 2) and did not recover even after the water treatment returned to well-watered conditions in the second stage of the experiment (Supplementary Figure 5.4). In well-watered conditions, there was a lower photosynthetic rate (Figure 5.2a, c), lower nocturnal titratable acidity (Figure 5.2b, d), smaller biomass (all P < 0.0001, Figure 5.1), and smaller relative aboveground plant water content (all $\mathrm{P}<0.0001$, Supplementary Figure 5.3) in C. imbricata in mixture with B. eriopoda than $C$. imbricata plants grown alone. Collectively, these results indicate that in well-watered conditions B. eriopoda had a strong competitive advantage over $C$. imbricata. The response of $C$. imbricata and B. eriopoda 
in both monoculture and mixture to altered $\mathrm{CO}_{2}$ and water conditions is summarized in Figure

5.3 .

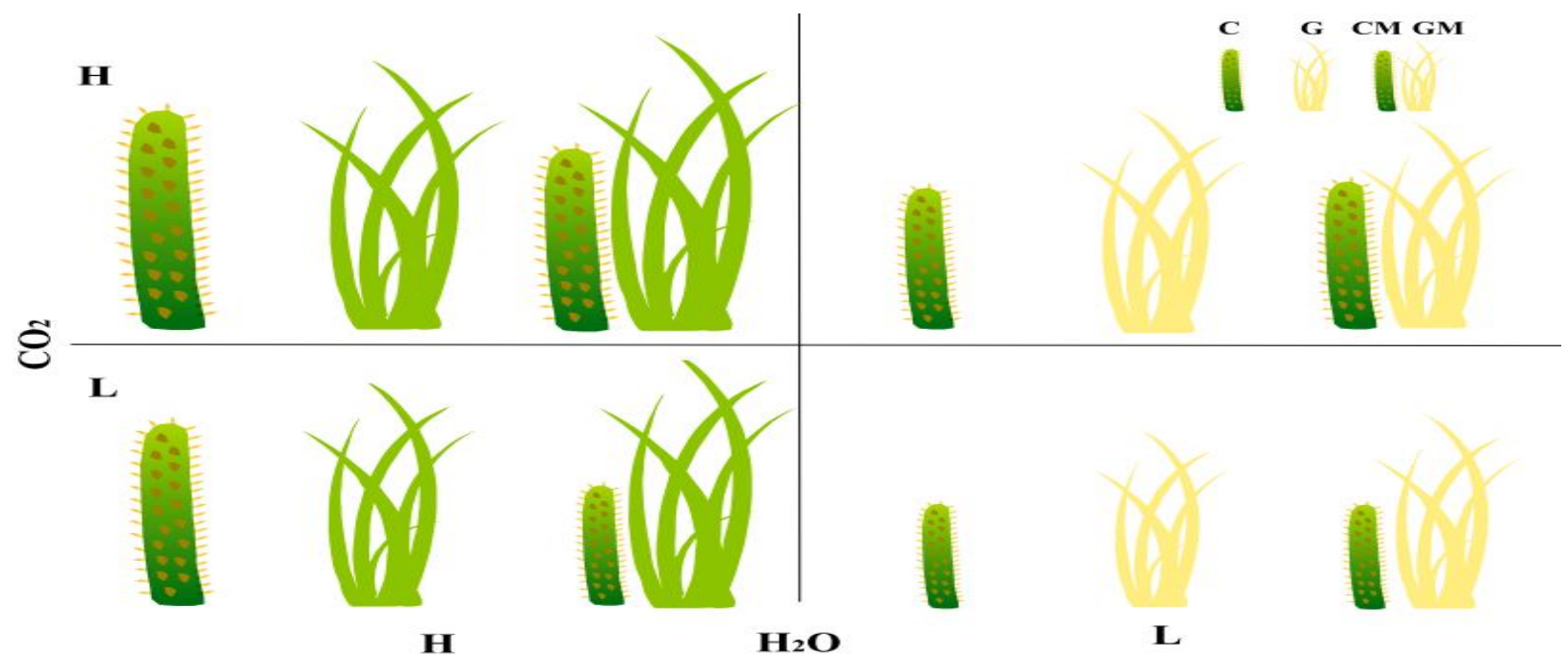

Figure 5-3: A conceptual representation of growth responses of CAM alone (C), grass alone (G), CAM-grass associations (CM, GM) to $\mathrm{CO} 2$ and water manupilations. $\mathrm{H}$ refers to high while $\mathrm{L}$ refers to low. The panel in the right and above indicates the initial stage of plants before $\mathrm{CO}_{2}$ and water treatments. Green color indicates that plants are alive while brown color indicates that plants are dead.

\subsection{Discussion}

As previously found for other $\mathrm{C}_{4}$ grasses (e.g., Smith et al., 2000; Morgan et al., 2001), $B$. eriopoda $\left(\mathrm{C}_{4}\right.$ grasses) showed a positive response to $\mathrm{CO}_{2}$ enrichment (Figure 5.1). The $\mathrm{C}_{4}$ photosynthesis performed at night by $C$. imbricata - which is typically thought to saturate quickly with increasing atmospheric $\mathrm{CO}_{2}$ concentrations (Poorter and Navas, 2003, Drennan and Nobel, 2000; Borland et al., 2009) - still had a positive response to $\mathrm{CO}_{2}$ enrichment (Figure 5.2), consistent with the response of most CAM species (Drennan and Nobel, 2000). This may occur because $\mathrm{CO}_{2}$ uptake at night in ambient $\mathrm{CO}_{2}$ conditions is still restricted by a low mesophyll conductance limiting the supply of $\mathrm{CO}_{2}$ to the photosynthetic tissue (Drennan and Nobel, 2000; Nelson and Sage, 2008; Ripley et al., 2013). The indirect effect of $\mathrm{CO}_{2}$ enrichment on 
photosynthesis and productivity - through a reduction in stomatal conductance and transpiration and an increase in water-use efficiency and soil moisture availability (Ainsworth and Long, 2005; Fay et al., 2012) - was significant for C. imbricata and B. eriopoda, as shown by the higher soil moisture availability found in well-watered conditions (Supplementary Figure 5.5), and the higher photosynthetic carbon isotope discrimination (lower stomatal conductance) (Supplementary Figure 5.6).

C. imbricata outcompeted B. eriopoda in drought conditions. C. imbricata coped with drought conditions by efficiently exploiting rainfall pulses and then drawing maximum benefit from storing the absorbed water (Lüttge, 2004; Borland et al., 2009). This led to higher relative aboveground plant water content (Supplementary Figure 5.3) and thus more favorable tissue water potential, lower water stress, and higher tolerance to droughts than B. eriopoda. Under drought conditions a more positive response to $\mathrm{CO}_{2}$ enrichment by $C$. imbricata indirectly disfavored B. eriopoda (Figure 5.1), which indicated that interspecific competition between CAM plants and $\mathrm{C}_{4}$ grasses can counteract the favorable direct effect of $\mathrm{CO}_{2}$ enrichment. Similarly, Suttle et al (2007) found that species interactions strongly influenced ecosystem responses to changing climate and reversed the direct climatic effects in a California grassland. Interspecific interactions slowed warming-induced upward movement of treelines on the Tibetan Plateau (Liang et al., 2016).

B. eriopoda had a strong competitive advantage over $C$. imbricata in well-watered conditions (Figures 5.1 and 5.2). As previously found for other grasses (e.g., Collins et al., 2012), B. eriopoda exhibited a high growth rate in well-watered conditions (Supplementary Figure 5.4) and an extensive root system with a high root/shoot ratio (Supplementary Figure 5.7). These traits allowed this grass to take advantage of the well-watered conditions under ambient $\mathrm{CO}_{2}$ levels, 
thereby indirectly limiting the response of $C$. imbricata to the increase in water availability (Figures 5.1 and 5.2). The intensive water usage strategies of grasses accounted for its competitive advantage over woody plants in savannas in response to increased intraannual or interannual rainfall variability (Xu et al., 2015; Yu et al., 2017). By comparison, C. imbricata had relatively high water use efficiency (Lüttge, 2004; Borland et al., 2009). Thus, there was likely a lower interspecific competition for soil water between $C$. imbricata and B. eriopoda than intraspecific competition among individuals of B. eriopoda, thereby leading to higher total biomass of B. eriopoda per individual in mixture than the case of B. eriopoda alone (Figures 5.1 and 5.3).

While only one CAM species was tested in this study, the ability to store water is a common characteristic of most CAM species (Lüttge, 2004; Borland et al., 2009) and a positive response of most CAM species to $\mathrm{CO}_{2}$ enrichment has been widely reported (Drennan and Nobel, 2000 2000; Poorter and Navas, 2003; Borland et al., 2009). Indeed, a meta-analysis of the literature shows that other studies have demonstrated that under elevated $\mathrm{CO}_{2}$ concentrations different families of CAM plants exhibited an average increase in daily $\mathrm{CO}_{2}$ uptake of $51 \%$ (Agavaceae), 17\% (Bromeliaceae), 71\% (Cactaceae), and 128\% (Crassulaceae) (Supplementary Figure 5.8a), while the increase in biomass averaged 54\% (Agavaceae), 6\% (Bromeliaceae), 34\% (Cactaceae), 31\% (Crassulaceae), 105\% (Orchidaceae) (Supplementary Figure 5.8b). Combined with our experimental results, these findings suggest that CAM plants could further expand their abundance under future climate change scenarios in regions affected by drought intensification (e.g., the southwestern U.S.) and elevated $\mathrm{CO}_{2}$ concentrations (Figure 5.3). While not investigated in this study, warming trends are expected to further benefit CAM plants because 
they exhibit maximum photosynthetic rates at higher temperatures (Borland et al., 2009; ReyesGarcía and Andrade, 2009).

This study provides the first experimental evidence that concurrent $\mathrm{CO}_{2}$ enrichment concentrations and water stress - the important drivers which have been found to greatly affect $\mathrm{C}_{3}$ or $\mathrm{C}_{4}$ plants (Smith et al., 2000; Poorter et al., 2003; Ainsworth and Long, 2005; Fay et al., 2012) - may allow CAM plants to outcompete co-occurring $\mathrm{C}_{4}$ plants. The physiological mechanisms underlying the competitive advantage of CAM plants are associated with its water storage, high drought tolerance, and lack of "saturation" in the response to $\mathrm{CO}_{2}$ enrichment. We suggest that understanding the potential shift of the competitive relationships between CAM plants and $\mathrm{C}_{4}$ grasses is crucial for evaluations of possible shifts in dryland vegetation composition, and related changes in ecosystem resilience and productivity, and the provision of ecosystem services under global environmental change. 


\title{
6 Crassulacean acid metabolism in Mesembryanthemum crystallinum: the effects of water stress, nutrient, and competition
}

This chapter is adapted from Yu KL, D’Odorico P, Carr D, Personius A, and Collins S, Crassulacean acid metabolism in Mesembryanthemum crystallinum: the effects of water stress, nutrient, and competition (under review).

\author{
Abstract \\ Crassulacean acid metabolism (CAM) is an evolutionary adaptation to water limited \\ environments and CAM plants are increasing their abundance in drylands worldwide. The \\ drivers and mechanisms, however, underlying CAM expression (nocturnal carboxylation) in \\ facultative CAM plants and the increased dominance of CAM plants remain poorly understood. I \\ investigate how nutrient and water conditions affect competition between Mesembryanthemum \\ crystallinum (facultative $\mathrm{CAM}$ ) and its interacting $\mathrm{C}_{3}$ species (Bromus mollis) found in \\ California's coastal grasslands and the extent to which water stress, nutrient, and competition \\ affect nocturnal carboxylation in $M$. crystallinum. High nutrient and low water conditions \\ favored M. crystallinum over B. mollis, in contrast to high water conditions. While low water \\ conditions induced CAM expression in 9 week old individuals of $M$. crystallinum, in these low \\ water treatments a $66 \%$ reduction in nutrient applied over the entire experiment did not increase \\ CAM expression. In high water conditions $M$. crystallinum both alone and in association with $B$. \\ mollis did not perform nocturnal carboxylation. I suggest that a high drought tolerance would \\ underlay the increased dominance by CAM plants in a future (drier) climate. Nocturnal \\ carboxylation that $M$. crystallinum typically uses to adapt to environmental stress could be \\ restricted by a strong competition in high water conditions.
}




\subsection{Introduction}

Crassulacean acid metabolism (CAM), a unique photosynthetic pathway evolving from $\mathrm{C}_{3}$ photosynthesis, is expressed by 6-7 \% of vascular plant species (Smith and Winter, 1996; Crayn et al., 2004). CAM plants feature nocturnal $\mathrm{CO}_{2}$ uptake, water storage, and a high water use efficiency (Lüttge, 2004; Borland et al., 2011). Crassulacean acid metabolism is an intriguing adaptation because of the photosynthetic plasticity and water conservation (Cushaman and Borland, 2002; Winter and Holtum, 2007), which provides CAM plants with ecological opportunities to increase their abundance under drier climate conditions (Drennan and Nobel, 2000; Cushaman and Borland, 2002; Borland et al., 2009; Reyes-García and Andrade, 2009).

Obligate CAM species perform CAM photosynthesis (i.e., nocturnal carboxylation) independently of environmental conditions, while the behavior of facultative CAM plants depends on environmental drivers (i.e., water stress) (e.g., Lüttge, 2004; Borland et al., 2011). Mesembryanthemum crystallinum, a halophytic annual in the Aizoaceae, is one of the most studied facultative CAM species. It can switch from $\mathrm{C}_{3}$ to $\mathrm{CAM}$ photosynthesis in response to environmental stress (i.e., low water and/or salinity) once it transitions from juvenile to adult ( $\approx>6-7$ weeks old) (Osmond, 1978; Winter and Holtum, 2007, 2014). Some studies suggest that the expression of CAM behavior in M. crystallinum is a preprogrammed developmental process related to ontogeny (Osmond, 1978; Adams, 1998; Cushaman and Borland, 2002). Others, however, demonstrate that CAM behavior in M. crystallinum is controlled by environmental conditions (i.e., water stress) (Piepenbrock and Schmitt, 1991; Winter and Holtum, 2007). In fact, $M$. crystallinum can revert from CAM back to $\mathrm{C}_{3}$ photosynthesis after removing 
environmental stress (Vernon et al., 1988; Schmitt, 1990; Winter and Holtum, 2014), although evidence of reversibility is difficult to determine because of the short life span of M. crystallinum leaves (Winter and Holtum, 2007, 2014).

Climate change studies predict an intensification of drought in many drylands around the world (e.g., Easterling et al., 2000; Dai et al., 2011; IPCC, 2013). Previous studies on CAM expression in M. crystallinum typically ceased watering for about two weeks until CAM expression was induced (e.g., Winter and Holtum, 2007, 2014). However, in the natural environments some sporadic rainfall events could still occur before CAM expression is induced in M. crystallinum. To date, it remains unclear whether less intense drought (e.g., a reduction in watering frequency instead of complete interruption of water applications) could induce CAM expression in M. crystallinum. Evidence of CAM expression of M. crystallinum induced by less intense drought may imply its adaptation to a wider range of environmental conditions.

Nutrient availability (mainly $\mathrm{N}$ ) is another important factor that may affect CAM expression, but its role remains poorly understood. Some studies in obligate CAM species indicate that $\mathrm{N}$ deficiency limited the rate of CAM photosynthesis (e.g., Winter et al., 1982; Nobel, 1983), presumably because of the $\mathrm{N}$ requirements by the enzymes used for plant photosynthesis. In contrast, other studies show that CAM plants (including both obligate and facultative) grown in conditions with lower $\mathrm{N}$ availability had a higher CAM expression (e.g., Ota et al., 1988; Paul and Cockburn, 1990; Lüttge, 2006; Winter and Holtum, 2011). Recent studies recognized the role of carbohydrates (i.e., 3-carbon acceptor phosphoenolpyruvate, PEP which is produced by degrading starch/sugars) as substrates in nocturnal carboxylation (Borland and Dodd, 2002; Antony and Borland, 2008; Antony et al., 2008). Haider et al (2012) found that CAM expression in a starch-deficient mutant of $M$. crystallinum was suppressed under high salt additions. Indeed, 
the only study to investigate the effects of $\mathrm{N}$ and $\mathrm{P}$ deficiency on CAM expression in $M$. crystallinum found that CAM expression increased (Paul and Cockburn, 1990). However, the N and $\mathrm{P}$ deficiency was applied at the adult stage, after the plants were able to accumulate a sufficient carbohydrates from earlier growth stages with no $\mathrm{N}$ and $\mathrm{P}$ limitation. It is unclear how nutrient treatments applied in early life stages and their interactions with water availability could affect CAM expression in M. crystallinum.

Water and nutrient conditions are likely to affect not only the physiology of CAM plants but also their competitive relationships with other functional groups (i.e., $\mathrm{C}_{3}$ and/or $\mathrm{C}_{4}$ plants). Research has mostly focused on the response of $\mathrm{C}_{3}$ and/or $\mathrm{C}_{4}$ plants and their competitive relationships to water and nutrient conditions (e.g., Niu et al., 2008; van der Waal et al., 2009). This study investigates the effects of nutrient and water conditions on competition between the CAM species $M$. crystallinum (invasive) and an interacting $\mathrm{C}_{3}$ species Bromus mollis. B. mollis is an annual $\mathrm{C}_{3}$ grass that has invaded the coastal grasslands of California, where it competes with M. crystallinum (McCown and Williams, 1968; Vivrette and Muller, 1977; D’Antonio, 1992). While the higher salt tolerance of M. crystallinum is often thought to be the key mechanism for its invasion success in this region (Vivrette and Muller, 1977), the role of other divers of global environmental change including $\mathrm{N}$ deposition and low soil water availability (Harpole et al., 2007; Suttle et al., 2007) remains poorly understood. CAM plants are more tolerant of low water availability because they can store water in aboveground biomass and they exhibit high water use efficiency (Lüttge, 2004; Borland et al., 2009). As a consequence, $M$. crystallinum would be expected to outcompete B. mollis under low soil water. In wet conditions, however, B. mollis $\left(\mathrm{C}_{3}\right.$ grasses) could sustain higher growth rates, especially with high $\mathrm{N}$ (McCown and Williams, 1968), and thus have a competitive advantage with respect to $M$. 
crystallinum in access to light, soil water and nutrients. M. crystallinum, on the other hand, may adapt to competition with B. mollis by switching to CAM photosynthesis. Testing these hypotheses would provide new insights into crassulacean acid metabolism as an adaptive strategy to both abiotic and biotic stress.

I developed greenhouse experiments in which the seedlings of M. crystallinum and B. mollis in both monoculture and mixtures were subjected to two nutrient levels ("high" and "low") and two water levels ("high" and "low"). Plant responses were evaluated through measurements of gas exchange, concentrations of titratable acidity, aboveground plant $\mathrm{N}$, biomass and productivity. I asked: (i) how does the exposure to moderate drought affect CAM expression and its reversibility in M. crystallinum? (ii) How does nutrient availability and its interactions with water availability influence CAM expression and reversibility in M. crystallinum? (iii) How does competition between M. crystallinum and B. mollis respond to nutrient and water conditions? (iv) Is the physiological plasticity in $M$. crystallinum an adaptive strategy for competition with $B$. mollis?

6.2 Materials and methods

\subsubsection{Experimental design}

M. crystallinum seeds were germinated in plastic trays covered with 1-2 mm substratum of mineral soil in the greenhouse facility at the University of Virginia. Likewise, seeds of B. mollis were germinated in plastic pots $(14.5 \mathrm{~cm}$ in diameter and $10.5 \mathrm{~cm}$ in height with a capacity of $1.3 \mathrm{~L})$ in the greenhouse. Germination started on Sep $6^{\text {th }} 2015$, and by Sep $20^{\text {th }} 2015$, seedlings of $M$. crystallinum and B. mollis were ready to be transplanted in either monoculture (one individual of 
M. crystallinum or 20 individuals of B. mollis) or a mixture (one individual of M. crystallinum and 20 individuals of $B$. mollis with $M$. crystallinum located in the middle of each pot).

The study used a randomized block experiment design in which the seedlings of $M$. crystallinum and B. mollis in both monoculture and mixture were subjected to two nutrient fertilization levels (high and low) under high and low water conditions. This experiment had three stages of plant harvest (Figure 6.1) and there were 6 replicates arranged in 6 blocks for each measurement in each stage.

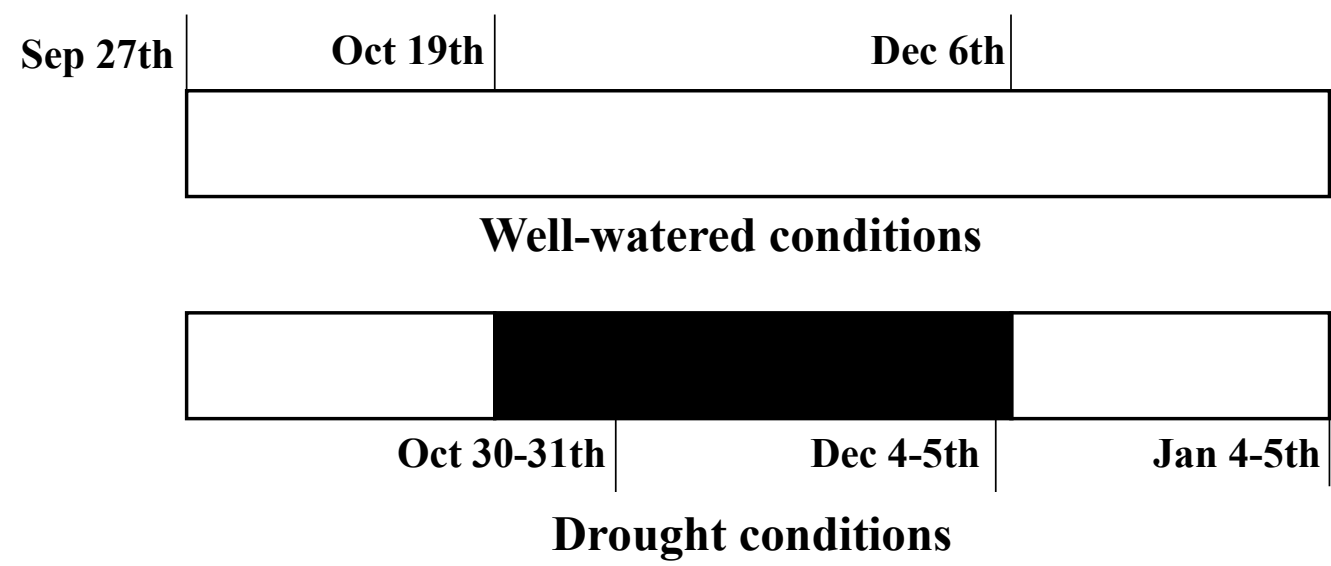

Figure 6-1: Schematic diagram of water treatments in high and low water conditions. Black zone represents low frequency watering treatment (once in every 8 days) while the white zone represents high frequency watering treatment (once in every 2 days). Plants were harvested in the first (Oct 30-31th), second (Dec 4-5th), and third (Jan 4-5th) stages of the experiment. Gas exchange and titratable acidity were measured 1-2 days before each harvest.

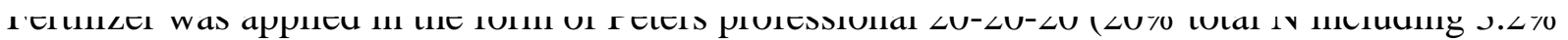

$\mathrm{NH}_{4}-\mathrm{N}, 5.3 \% \mathrm{NO}_{3}-\mathrm{N}$, and $11.5 \%$ urea, $20 \% \mathrm{P}_{2} \mathrm{O}_{5}, 20 \% \mathrm{~K}_{2} \mathrm{O}$, as well as other micronutrients).

Each pot in high nutrient conditions received $15 \mathrm{mg} \mathrm{N}$ once every 8 days while each pot in low nutrient conditions received $15 \mathrm{mg} \mathrm{N}$ once every 24 days. Plants in high water conditions were watered every two days with an intensity of $8 \mathrm{~mm}$ per event during the whole experiment. Plants in low water conditions were watered every two days with an intensity of $8 \mathrm{~mm}$ per event until 
Oct $19^{\text {th }} 2015$ and were then watered every 8 days with an intensity of $8 \mathrm{~mm}$ per event until Dec $6^{\text {th }} 2015$, when the water treatment returned to high water conditions (i.e., watering frequency of once every two days) until the end of the experiments (Jan $\left.5^{\text {th }} 2015\right)$; thus, this low water treatment was in the form of a "wet-dry-wet" sequence (Figure 6.1). I note that, in contrast to previous studies (Winter and Holtum, 2007, 2014), the low water treatment entailed a low watering frequency instead of complete interruption of water applications. Plants were subjected to well controlled light (i.e., 12 hours light with photosynthetically photon flux density (PPFD) of $700-800 \mathrm{umol} \mathrm{m}^{-2} \mathrm{~s}^{-1}$ from 7 am to $7 \mathrm{pm} \mathrm{EDT)} \mathrm{and} \mathrm{temperature} \mathrm{(i.e.,} 25^{\circ} \mathrm{C}$ during the day and $20^{\circ} \mathrm{C}$ during the night) conditions.

\subsubsection{Gas exchange and concentration of titratable acidity}

Before plant harvest (usually two days before), gas exchange for M. crystallinum and B. mollis in both monoculture and mixture were measured using the standard leaf chamber $\left(2 \times 3 \mathrm{~cm}^{2}\right)$ in a Licor 6400 gas analyzer. During the day the measurements of gas exchange were made for $M$. crystallinum and B. mollis between 10:00 am and 12:00 pm at a constant leaf temperature of $23{ }^{\circ} \mathrm{C}$ and photosynthetic active radiation of $1500 \mu \mathrm{mol} \mathrm{m} \mathrm{m}^{-2} \mathrm{~s}^{-1}$; during the night gas exchange was measured once every two hours between $8 \mathrm{pm}$ and 8 am on the following day for $M$. crystallinum - both alone and mixed with B. mollis - at a constant leaf temperature of $23{ }^{\circ} \mathrm{C}$ and photosynthetic active radiation of $0 \mu \mathrm{mol} \mathrm{m} \mathrm{m}^{-2} \mathrm{~s}^{-1}$. Gas exchange during the day was measured once while during the night it was measured to capture the nocturnal change of $\mathrm{CO}_{2}$ uptake.

After measurements of gas exchange (still before plant harvest), one leaf of $M$. crystallinum (alone or mixed with $B$. mollis) was sampled from each plant at 7 am and $5 \mathrm{pm}$, respectively, and then stored at $-20^{\circ} \mathrm{C}$ before being used for measurements of titratable acidity. Titratable acidity was measured using the acid base titration method (von Caemmerer and Griffiths, 2009), 
whereby leaf discs $\left(4 \mathrm{~cm}^{2}\right)$ are boiled in $1.5 \mathrm{~mL} \mathrm{H}_{2} \mathrm{O}$ for $5 \mathrm{~min}$ in a microfuge tube; $10 \mathrm{mM}$ $\mathrm{NaOH}$ was added into the same tube with $20 \mathrm{uL}$ of a $1 / 5$ dilution of phenolphthalein as indicator. Concentration of titratable acidity was then calculated from the amount of $\mathrm{NaOH}$ added (von Caemmerer and Griffiths, 2009).

\subsubsection{Light availability and biomass measurements}

Before plant harvest, light intensity in mixture was measured above and at the bottom of canopies (approximately at ground level) using a HOBO Pendant ${ }^{\circledR}$ Temperature/Light $64 \mathrm{~K}$ Data Logger. Relative light intensity (\%) was calculated as the ratio of light intensity under canopies to that above canopies (Sun et al., 2015). Plants were harvested on Oct $30-31^{\text {th }}$ (the first stage), Dec $4-5^{\text {th }}$ (the second stage), and Jan $4-5^{\text {th }}$ (the third stage), respectively with 6 replicates in each block in each stage (Figure 6.1). M. crystallinum and B. mollis in mixture were separated; loose roots found in the soil profile not attached to the parent plant ( $<5 \%$ of total root biomass) were classified as belonging to M. crystallinum or B. mollis based on root color, diameter and shape. Roots were washed free of soil through 0.1-mm mesh sieves. Plant tissues were dried at $60^{\circ} \mathrm{C}$ for $72 \mathrm{~h}$ and weighed. Total biomass and shoot-to-root biomass ratios were calculated. Note that the samples of fresh $M$. crystallinum collected for measurements of titratable acidity were weighed and then converted to dry biomass using the fresh/dry biomass ratio.

\subsubsection{Soil moisture, plant leaf water potential and plant $\mathrm{N}$}

Soil moisture content was determined gravimetrically by drying samples at $60^{\circ} \mathrm{C}$ for $72 \mathrm{~h}$ after plants were harvested. Plant leaf water potential was measured using a Decagon WP4 ${ }^{\circledR}$ potentiometer. Plant samples dried at $60^{\circ} \mathrm{C}$ for $72 \mathrm{~h}$ were ground and homogenized for elemental analysis. Plant N analysis was performed using a Thermo Scientific FLASH 2000 NC Analyzer. 


\subsubsection{Statistical analysis}

The effects of nutrient treatment, water availability, species competition and time, as well as their interactions, on plant leaf water potential, specific leaf area, diurnal photosynthetic assimilation, total biomass, belowground to aboveground biomass ratio, and aboveground plant total $\mathrm{N}$ were analyzed using a five-way ANOVA with block as a random factor. The effects of nutrient, water, species, and time as well as their interactions on soil moisture were analyzed using a four-way ANOVA with block as a random factor. The BA values were natural log transformed prior to ANOVA. In general, the most interesting effects were found in multiway interactions. To explore these interactions, I constructed pairwise orthogonal contrasts to detect differences between individual pairs of means. All statistics were performed in SAS 9.4.

\subsection{Results}

\subsubsection{Leaf water potential (LWP)}

Nutrient availability had significant effects on leaf water potential (LWP) $(\mathrm{P}=0.0075$,

Supplementary Table 1). In fact, in the first stage there was a sharp decrease in LWP of B. mollis $\left(\mathrm{C}_{3}\right.$ grass $)$ both alone $(\mathrm{G})$ and in mixture $(\mathrm{GM})$ in high nutrient and low water conditions (HNLW) (LWP $=-10.8 /-11.3 \mathrm{MPa}$ for $\mathrm{G} / \mathrm{GM}$ ) as compared to low nutrient and low water conditions (LNLW) $(\mathrm{LWP}=-7.6 /-6.8 \mathrm{MPa}$ for G/GM) $(\mathrm{P}<0.0001$, Figure 6.2a). This result as well as the fact that $B$. mollis died earlier in HNLW than LNLW after watering frequency was reduced indicates that $B$. mollis was more vulnerable to low water and high nutrient conditions. Nutrient availability, however, did not significantly affect LWP in any vegetation type $(M$. 
crystallinum alone-FC, $M$. crystallinum mixed with $B$. mollis-FCM, G, GM) in high water conditions in any of the three stages (all $\mathrm{P} \geq 0.1400$, Figure 6.2).

(a)
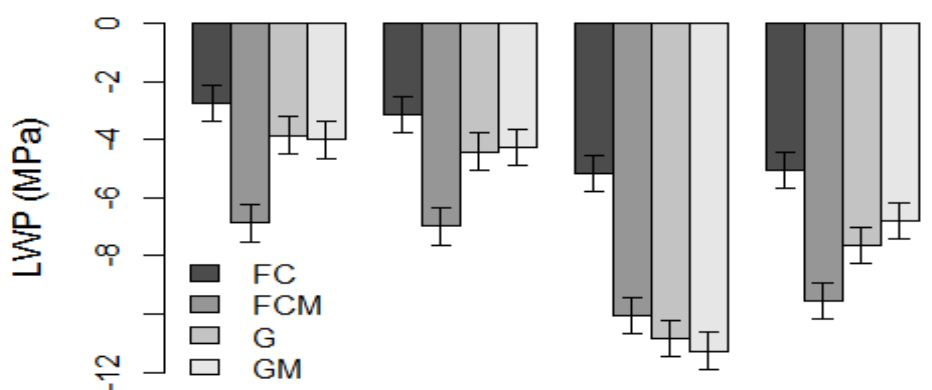

(b)
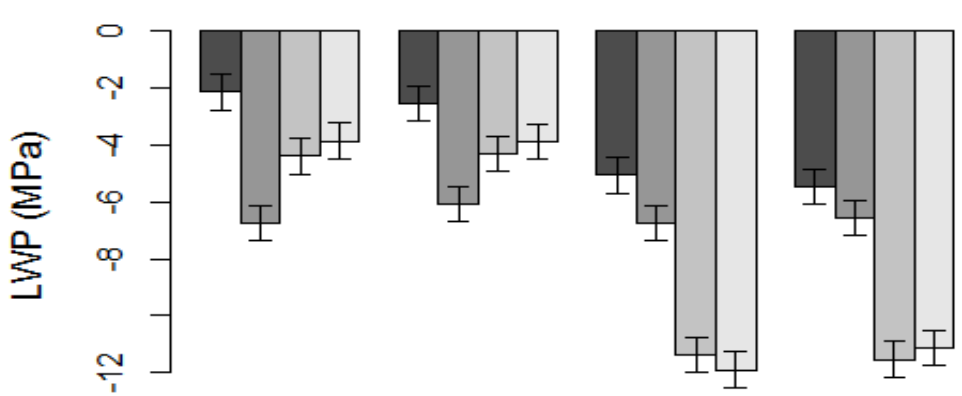

(c)

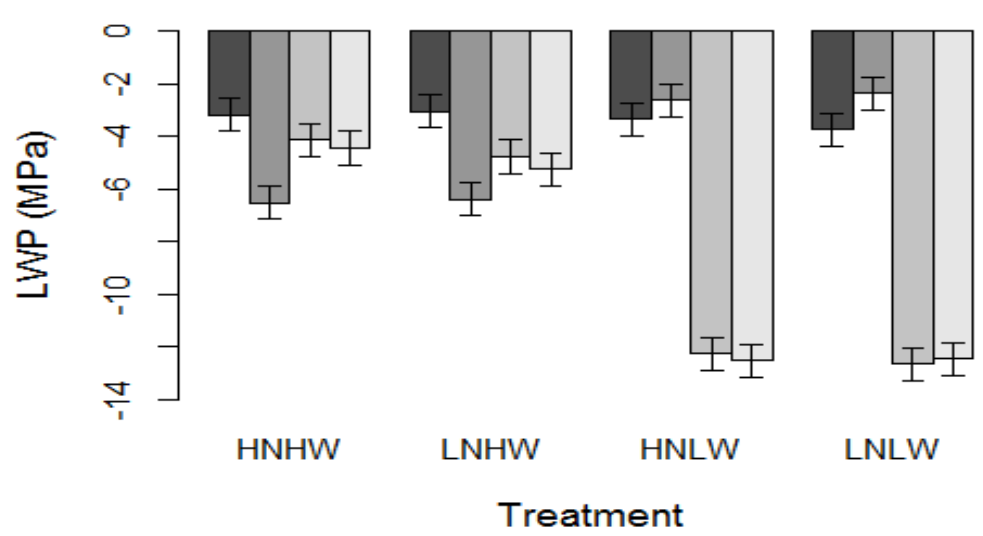

Figure 6-2: Leaf water potential (LWP) in Mesembryanthemum crystallinum alone (FC), Mesembryanthemum crystallinum in mixture (FCM), Bromus mollis alone (G), Bromus mollis in mixture (GM) under different nutrient and water conditions in the first (a), second (b), an and third (c) stages of the experiment. HNHW refers to high nutrient and high water conditions; LNHW refers to low nutrient and high water conditions; HNLW refers to high nutrient and low water conditions; LNLW refers to low nutrient and low water conditions. Each bar represents the mean of 6 values while error bar indicates $95 \%$ confidence intervals.

LWP of all vegetation types was significantly greater in high water conditions than low water conditions (all $\mathrm{P}<0.0001$, Figure 6.2) except for the cases of M. crystallinum (CAM) alone and 
M. crystallinum in mixture in the third stage which was a high water treatment (both $\mathrm{P} \geq 0.1204$ for CAM alone; both $\mathrm{P}<0.0001$ for CAM in mixture, Figure 6.2c). LWP in M. crystallinum alone was significantly greater than in B. mollis alone and B. mollis in mixture over all the stages of the experiments (all $\mathrm{P} \leq 0.0158$, Figure 6.2 ). LWP of M. crystallinum in mixture was significantly lower than M. crystallinum alone because of competition for soil water from $B$. mollis (all $\mathrm{P} \leq 0.0140$, Figure 6.2), except for the case of low water conditions because of lack of competition in the third stage ( $\mathrm{P} \geq 0.1011$, Figure 6.2c); in fact, $B$. mollis both alone and in mixture died at some time between the first and second stage, as showed by its extremely low LWP (Figure 6.2a, b), and the highest soil moisture in B. mollis alone (Supplementary Figure 6.1), and lack of photosynthetic assimilation (Figure 6.3a, b) in the second and third stages. Overall, these results indicate that high nutrient and low water conditions favor M. crystallinum over B. mollis.

\subsubsection{Photosynthetic assimilation during the day $\left(A_{D}\right)$}

The effect of nutrient availability on photosynthetic assimilation during the day $\left(A_{D}\right)$ depends on water conditions (Supplementary Table $1 ; \mathrm{P}<0.001$ for $\mathrm{N} \times$ water). Similar to the pattern of specific leaf area (Supplementary Figure 6.2), in high water conditions $A_{D}$ of all vegetation types in high nutrient conditions was significantly greater than low nutrient conditions (all $\mathrm{P} \leq 0.0387$, Figure 6.3); the exceptions, however, are the cases of M. crystallinum in mixture in the second and third stages $(\mathrm{P}=0.5181$ for the second stage; $\mathrm{P}=0.3458$ for the third stage; Figure $6.3 \mathrm{~b}, \mathrm{c})$ where competition outweighed the nutrient effects. In low water conditions, $A_{D}$ of all vegetation types in high nutrient conditions was not significantly different from low nutrient conditions (all $\mathrm{P} \geq 0.1675$, Figure 6.3) except the third stage (all $\mathrm{P} \leq 0.0412$ for all vegetation types in high water conditions and FC and FCM in low water conditions, Figure 6.3c). $A_{D}$ in high water 
conditions was significantly greater than in low water conditions (all $\mathrm{P} \leq 0.0071$, Figure 6.3)

except for the cases of M. crystallinum in mixture in the second stage in which competition from grasses was lacking $(\mathrm{P}<0.0001$, Figure $6.3 \mathrm{~b})$ and M. crystallinum alone and in mixture in the third stage ( $\mathrm{P}=0.0234$ for $\mathrm{FC} ; \mathrm{P}<0.0001$ for $\mathrm{FCM}$, Figure $6.3 \mathrm{c})$. $\mathrm{A}_{\mathrm{D}}$ of M. crystallinum alone was significantly higher than $M$. crystallinum in mixture (all $\mathrm{P} \leq 0.0029$, Figure 6.3 ) except in low water conditions in the second and third stages $(\mathrm{P}=0.0080$ for the second stage; $\mathrm{P}<0.0001$ for the third stage, Figure $6.3 \mathrm{~b}, \mathrm{c})$. $A_{D}$ of $B$. mollis alone was not significantly different from $B$. mollis in mixture in all the treatments over the three stages (all $\mathrm{P} \geq 0.3775$ ) (Figure 6.3).

(a)

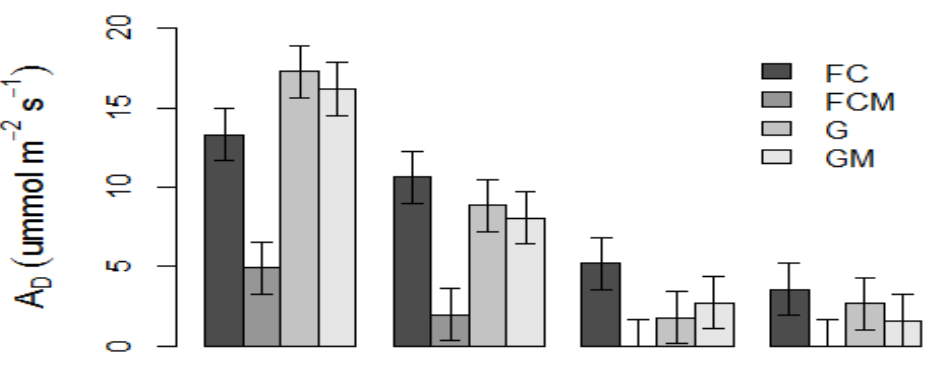

(b)

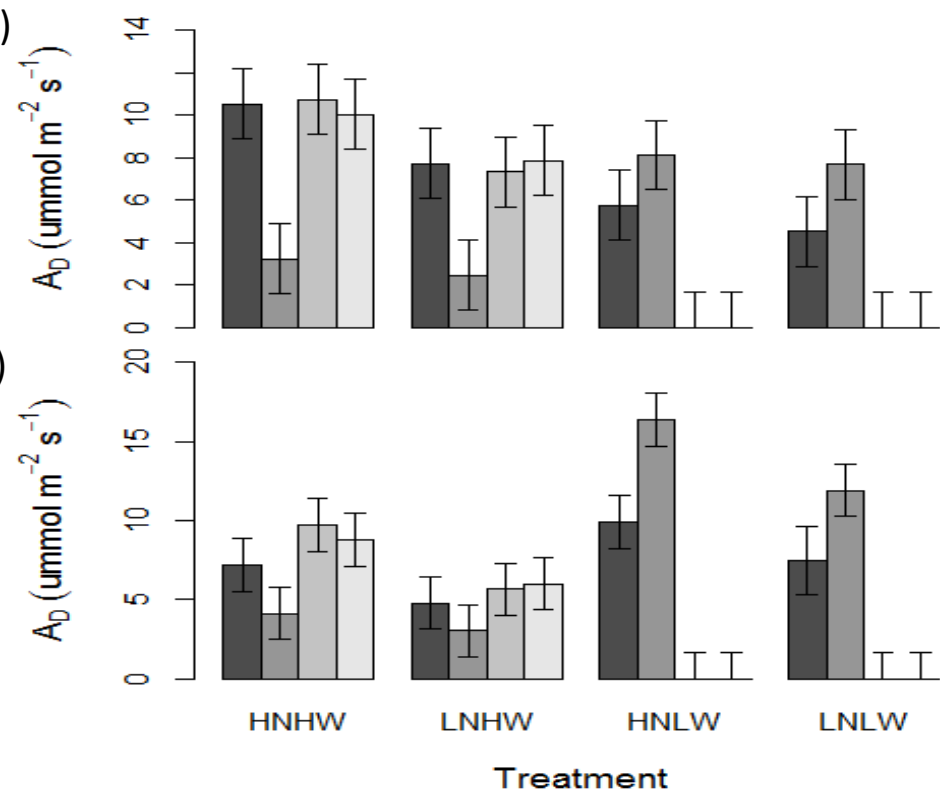

Figure 6-3: Photosynthetic assimilation during the day (AD) in Mesembryanthemum crystallinum alone (FC), Mesembryanthemum crystallinum in mixture with B. mollis (FCM), Bromus mollis alone (G), Bromus mollis in mixture with M. crystallinum (GM) under different nutrient and water conditions in the first (a), second (b), and third (c) stages of the experiment. Symbols for each treatment are the same as Figure 6.2. Each bar represents the mean of 6 values while error bar indicates $95 \%$ confidence intervals. 


\subsubsection{Total Biomass (TB)}

There was a significant effect of nutrient and water interactions on total biomass (TB) (Supplementary Table 1; $\mathrm{P}<0.001$ ). For high water conditions, increase in nutrient significantly increased TB of M. crystallinum alone (FC), B. mollis alone and B. mollis in mixture in all the three stages (all $\mathrm{P} \leq 0.0006$, Figure 6.4) except the case of $\mathrm{FC}$ in the first stage $(\mathrm{P}=0.122$, Figure 6.4a); in low water conditions increase in nutrient significantly increased TB of M. crystallinum alone in the third stage (which is indeed a high water treatment) $(\mathrm{P}=0.0004$, Figure $6.4 \mathrm{c})$, in contrast to other cases (all $\mathrm{P} \geq 0.2667$, Figure 6.4 ). Increase in water significantly increased biomass of $B$. mollis alone and $B$. mollis in mixture in all the three stages (all $\mathrm{P} \leq 0.0184$, Figure 6.4) while the effect of water additions on TB of $M$. crystallinum alone was significant in the second and third stages $(\mathrm{P}<0.0001$ for the second stage; $\mathrm{P}<0.0001$ for the third stage, Figure $6.4 \mathrm{a}, \mathrm{b})$ but not the first stage $(\mathrm{P}=0.3723$, Figure $6.4 \mathrm{a})$. Over the three stages, TB of $M$. crystallinum alone ( $\mathrm{TB}=3.3,6.6,9.6 \mathrm{~g}$ for the first, second, and third stages, respectively) was significantly greater than $M$. crystallinum in mixture $(\mathrm{TB}=0.23,0.28,0.45 \mathrm{~g}$ for the first, second, and third stages, respectively; all $\mathrm{P}<0.0001$, Figure 6.4). That is, under high water conditions B. mollis outcompeted M. crystallinum for light (Supplementary Figure 6.3) and soil nitrogen (Supplementary Figure 6.4). In high water conditions TB of B. mollis alone and B. mollis in mixture significantly increased with time (all $\mathrm{P}<0.0001$, Figure 6.4 ), in contrast to low water conditions. The effects of nutrient and water conditions on TB of M. crystallinum in mixture were not significant (all $\mathrm{P} \geq 0.8514$, Figure 6.4) in the first and second stages where competition from B. mollis outweighed the nutrient and water effects. Consistent with the pattern of $A_{D}$, these results of TB collectively show that nutrients and water co-limit the productivity of 
M. crystallinum, and $M$. crystallinum has a competitive advantage relative to B. mollis in low water conditions.

(a)

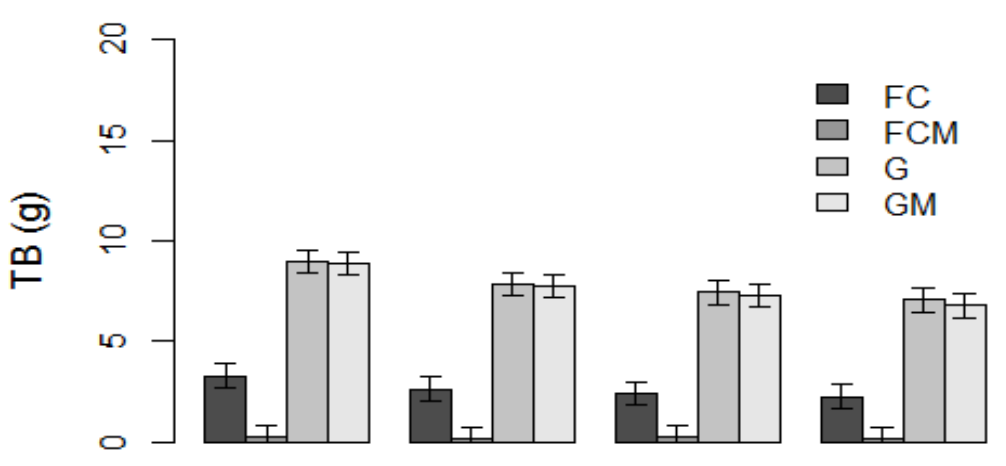

(b)

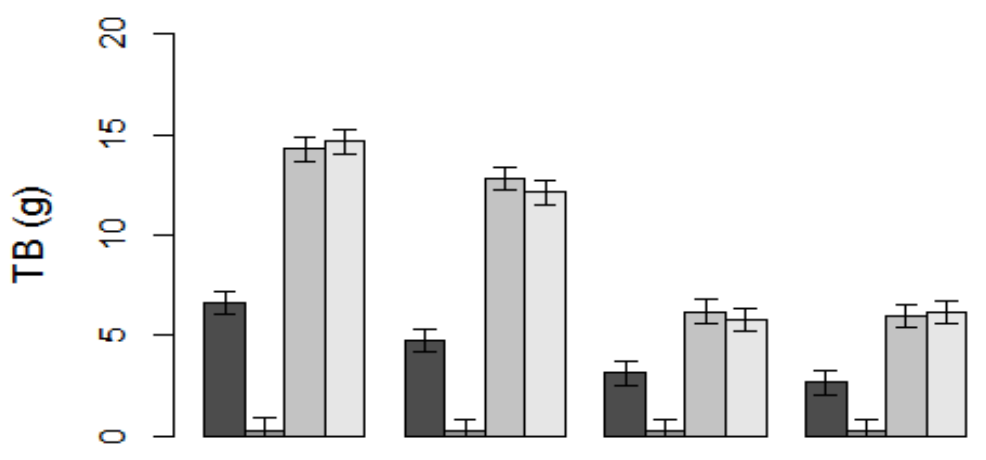

(c)

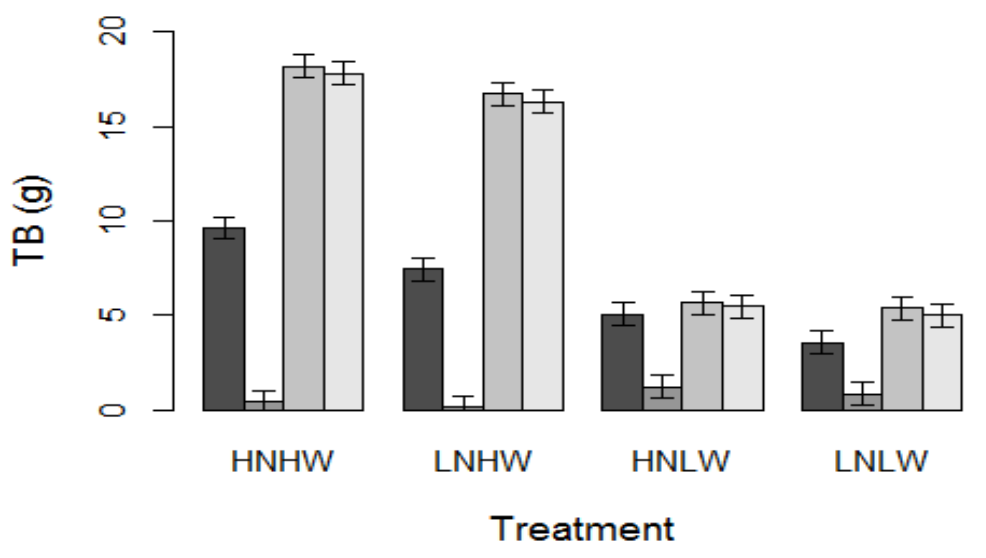

Figure 6-4: Total biomass (TB) in Mesembryanthemum crystallinum alone (FC), Mesembryanthemum crystallinum in mixture (FCM), Bromus mollis alone (G), Bromus mollis in mixture (GM) under different nutrient and water conditions in the first (a), second (b), and third (c) stages of the experiment. Symbols for each treatment are the same as Figure 6.2. Each bar represents the mean of 6 values while error bar indicates $95 \%$ confidence intervals. 
6.3.4 Nocturnal photosynthetic assimilation $\left(\mathrm{A}_{\mathrm{N}}\right)$ and titratable acidity $(\mathrm{TA})$

M. crystallinum (either alone or mixed with B. mollis grasses) did not perform CAM expression (nocturnal carboxylation) in the first stage of any of the treatments, as indicated by the negative values of nocturnal photosynthetic assimilation $\left(\mathrm{A}_{\mathrm{N}} \approx-1-2 \mathrm{ummol} \mathrm{m}^{-2} \mathrm{~s}^{-1}\right)$ and the lack of nocturnal accumulation of titratable acidity (see legend in Figure 6.5).
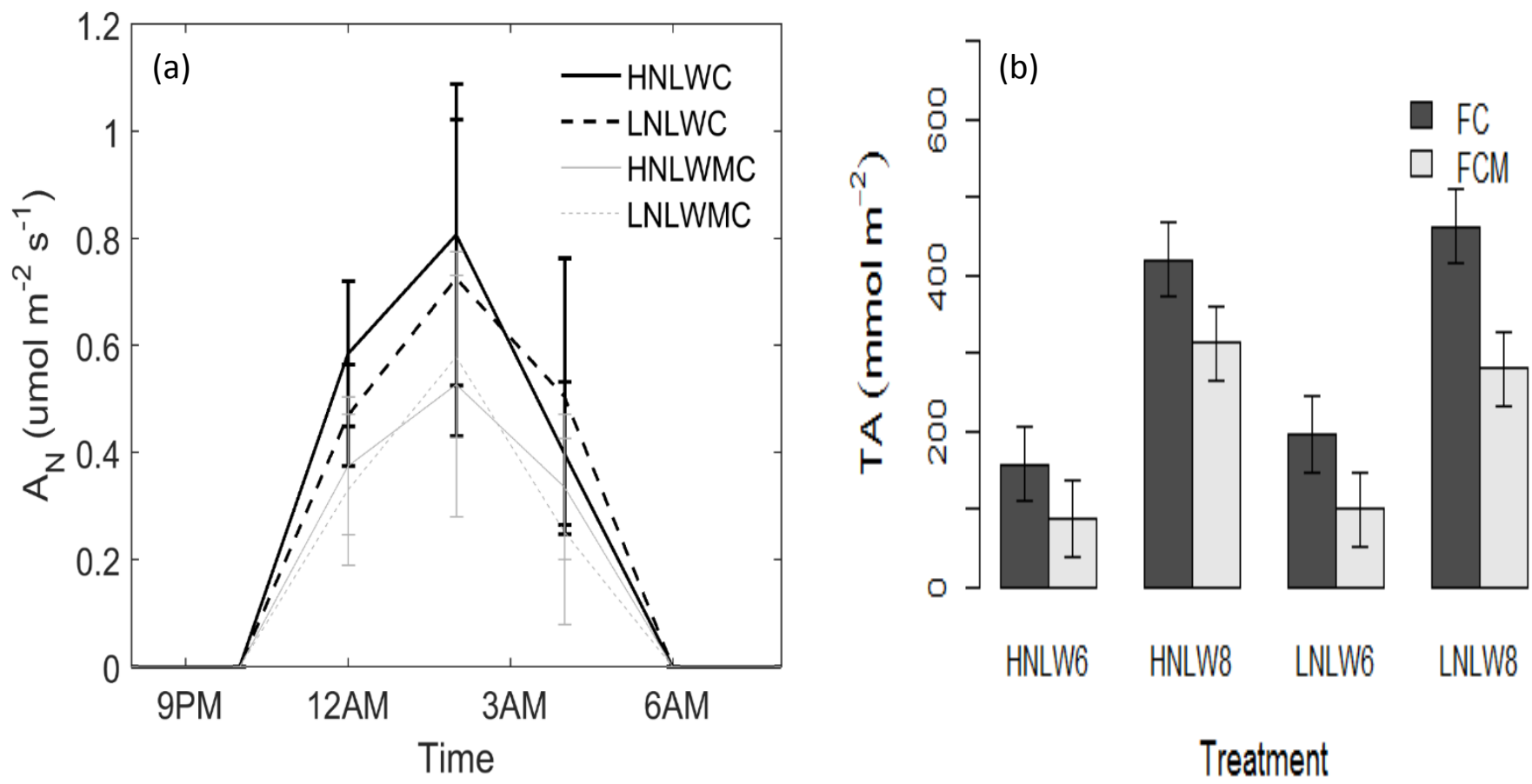

Figure 6-5: Nocturnal change of photosynthetic assimilation (AN) of Mesembryanthemum crystallinum in alone (FC) and mixture (FCM) in low water conditions in the second stage of the experiment. Symbols for each treatment are the same as in Figure 6.2. (b) Titratable acidity (TA) of Mesembryanthemum crystallinum in alone (FC) and mixture (FCM) in low water conditions in the second stage. 6 refers to $6 \mathrm{pm}$ while 8 refers $8 \mathrm{am}$. Each bar represents the mean of 6 values while error bar indicates $95 \%$ confidence intervals. Both FC and FCM do not express CAM behavior in any treatments during the first and third stages of the experiment, and thus values of $A_{N}$ and TA in these two stages are not shown. 
In the second stage, the $\mathrm{A}_{\mathrm{N}}$ of $M$. crystallinum (both alone and in mixture) was also negative $(\approx$ $-1-2$ umol m $\mathrm{m}^{-2} \mathrm{~s}^{-1}$ ) in high water conditions, which was consistent with the fact there was no significant difference of titratable acidity between late afternoon $\left(\mathrm{TA}=48.08 / 53.98 \mathrm{mmol} \mathrm{m}{ }^{-2}\right)$ and early morning $\left(\mathrm{TA}=47.61 / 51.37 \mathrm{mmol} \mathrm{m}^{-2}\right)$ (see legend in Figure 6.5). These results indicate that M. crystallinum in mixture did not switch to CAM photosynthesis in response to strong competition with B. mollis. In contrast, low water treatments in both high nutrient and low nutrient conditions led to a positive $\mathrm{A}_{\mathrm{N}}\left(\mathrm{A}_{\mathrm{N}} \approx 0.5-0.8 \mathrm{ummol} \mathrm{m}^{-2} \mathrm{~s}^{-1}\right.$ by $M$. crystallinum alone and $\mathrm{A}_{\mathrm{N}} \approx 0.3-0.6$ ummol m $\mathrm{m}^{-2} \mathrm{~s}^{-1}$ by M. crystallinum in mixture) at 1-4 am (Figure 6.5a) as well as greater TA in early morning than late afternoon (all $\mathrm{P}<0.0001$, Figure $6.5 \mathrm{~b}$ ), which indicated CAM expression. Reduction of nutrient application in M. crystallinum (alone or in mixture) did not significantly affect its $A_{N}$ and $T A$ in late afternoon and early morning in low water treatments (all $\mathrm{P} \geq 0.2548$, Figure 6.5). Consistent with the pattern of $\mathrm{A}_{\mathrm{N}}, \mathrm{TA}$ in $M$. crystallinum in mixture was significantly lower than in M. crystallinum alone in both late afternoon and early morning in both HNLW and LNLW (both $\mathrm{P} \leq 0.0411$, Figure 6.5).

\subsection{Discussion}

Rainfall regimes are expected to change (e.g., increase in drought occurrence in drylands, or decrease in the frequency of rainy days) and $\mathrm{N}$ deposition to increase in the next few decades. These changes are likely to affect $\mathrm{C}_{3}$ and/or $\mathrm{C}_{4}$ plants and their competitive relationships (e.g., Niu et al., 2008; van der Waal et al., 2009). It remains unclear, however, how these environmental change drivers can affect the competitive relationship between CAM plants and other functional groups (i.e., $\mathrm{C}_{3}$ and/or $\mathrm{C}_{4}$ plants). This study investigated the ecophysiological mechanisms underlying the potential advantage of a model facultative CAM species ( $M$. crystallinum) while interacting with $\mathrm{C}_{3}$ species (B. mollis) found in California's coastal 
grasslands under nutrient and water manipulations. I found that because of its drought tolerance M. crystallinum had a competitive advantage relative to $B$. mollis in low water and $\mathrm{N}$ deposition conditions. Less intensive drought associated with a reduction in rainfall frequency (i.e., once every 8 days) induced CAM expression in M. crystallinum regardless of nutrient levels. In high water conditions, however, B. mollis was a stronger competitor for soil nutrients and light resources and this strong competition restricted the ability of $M$. crystallinum to switch to CAM expression - a type of physiological plasticity $M$. crystallinum typically uses to adapt to environmental stress and increase seed production and plant fitness.

6.4.1 CAM expression and reversibility in M. crystallinum as affected by less intensive droughts

In the experiment CAM expression in M. crystallinum occurred under moderate drought (i.e., watering once every eight days) (Figure 6.5). The drought treatment used in this study differs from those in previous studies that completely interrupted water applications until CAM expression was induced (Winter and Holtum, 2007, 2014), typically after 10 days. Thus, water in this study was supplied before CAM expression could be induced in response to water deficit. Had M. crystallinum been able to rapidly respond to water supply, it would not have performed CAM expression throughout second stage of the experiment, a phenomenon that was not observed in this study (Figure 6.5). Consistent with other studies (e.g., Cushman and Borland, 2002; Winter and Holtum, 2014), my results suggest a lack of rapid CAM response in $M$.

crystallinum to water supply/deficit. This characteristic is likely due to the seasonal pattern of $M$. crystallinum, which typically germinates and grows during the rainy season and then adapts to a relatively long summer dry season by performing CAM photosynthesis (e.g., Adams et al., 1998, Cushman and Borland, 2002). 
In this study consistent with others CAM expression in M. crystallinum was the result of environmental controls in response to droughts(Vernon et al., 1988, Schmitt, 1990, Piepenbrock and Schmitt, 1991, Winter and Holtum, 2007, Winter and Holtum, 2014). This was demonstrated both by the lack of CAM expression in M. crystallinum during the whole three stages in high water conditions, and the reversibility of CAM expression after returning to high water conditions in low water treatments.

6.4.2 The role of nutrients and their interactions with water availability in the expression and reversibility of CAM assimilation in $M$. crystallinum

Environmental stresses (i. e., drought and/or salt additions) are common drivers of CAM expression in M. crystallinum (Piepenbrock and Schmitt, 1991, Winter and Holtum, 2007, 2014), while the role of nutrients and their interactions with water limitations remain poorly investigated. Previous studies investigated the effects of $\mathrm{N}$ deficiency and its interactions with light on CAM expression in CAM plants. In some studies $\mathrm{N}$ deficiencies had a negative and in others a positive effect on CAM photosynthesis (Winter et al., 1982, Nobel, 1983, Ota et al., 1988).

My study shows that in low water conditions there was no significant difference in CAM expression by M. crystallinum between high nutrient and low nutrient conditions (Figure 6.5).This result is a sign that the availability of sufficient carbohydrates as substrates (i.e., 3carbon acceptor phosphoenolpyruvate, PEP, which is usually degraded by starch/sugars) also play important roles in CAM expression (Antony and Borland, 2008, Antony et al., 2008). CAM expression under salt stress was found to be suppressed in starch-deficient mutant of $M$. crystallinum (Haider et al., 2012) likely because, to maintain metabolism and growth, plants need to partition carbohydrates into other sinks which compete with the substrate requirement by 
nocturnal carboxylation (Borland and Dodd, 2002). Paul and Cockburn (1990) applying adequate $\mathrm{N}$ and $\mathrm{P}$ supply to young seedlings, leading to sufficient photosynthesis and carbohydrate accumulation, found a positive response of CAM expression in adults of M. crystallinum to $\mathrm{N}$ and P deficiency. In my study, low nutrient level (one third of high nutrient conditions) was applied during all stages; in low water conditions photosynthesis and production of carbohydrate are mainly limited by soil moisture instead of nutrients (Figure 6.3; Figure 6.4), implying that low nutrient supply is still adequate relative to water conditions and could sustain sufficient carbohydrate for nocturnal carboxylation. Although low nutrient supply was a limiting factor of plant photosynthesis and productivity in high water conditions (Figure 6.3; Figure 6.4), lack of water stress led to lack of CAM expression in M. crystallinum (Figure 6.5) (Osmond, 1978, Winter and Holtum, 2007, 2014). These results were consistent with the studies that showed that CAM was best expressed in facultative Kalanchoe lateritia at moderately low $\mathrm{N}$ conditions (i.e., with $\mathrm{N}$ applications $20 \%$ of the reference rate) as compared with ambient and very low $\mathrm{N}$ availability, likely because sufficient carbohydrate and environmental stress (i.e., $\mathrm{N}$ deficiency) were both satisfied under moderately low N (Santos and Salema, 1991, 1992).

6.4.3 The effects of nutrient and water conditions on competition between $M$. crystallinum and B. mollis

I found that $M$. crystallinum outcompeted $B$. mollis in low water treatments regardless of nutrient availability (Figure 6.2; Figure 6.3; Figure 6.4). The death of B. mollis in low water treatments was mainly caused by its intolerance to water stress exacerbated by the relatively high density (20 individuals per pot) and high biomass accumulated during prior high water conditions, which led to high evapotranspiration. As compared to B. mollis, M. crystallinum had much higher leaf water potential (Figure 6.2), and was more tolerant to water stress likely because of its ability to 
store the absorbed water in aboveground biomass (Lüttge, 2004, Borland et al., 2009). Higher salt tolerance of $M$. crystallinum could account for its competitive advantage and ability to invade coastal grasslands based on prior research (Vivrette and Muller, 1977), low water conditions could improve the competitive advantage of $M$. crystallinum with respect to $B$. mollis based on the experiment. Because droughts are predicted to become more intense across this region (e.g., Easterling et al., 2000, IPCC, 2013), dominance by CAM plants, such as $M$. crystallinum, will likely increase.

In addition to drought intensification, another driver of environmental change is increased $\mathrm{N}$ deposition. Mediterranean ecosystems such as California grasslands where $M$. crystallinum and B. mollis interact could be particularly vulnerable to impacts from climate change and $\mathrm{N}$ deposition (e.g., Parton et al., 1994, Sala et al., 2000). Increased nutrient availability and low water availability acted in concert to affect the competitive relationship between $M$. crystallinum and B. mollis. In fact, I found that after ceasing water applications (stage 1 of the experiment) the leaf water potential of $B$. mollis - both alone and in mixture - was much lower with high rates of nutrient supply than in low nutrient conditions (Figure 6.3a); I also observed that B. mollis in high nutrient conditions died earlier $(\approx 1-2$ weeks $)$ than in low nutrient conditions in response to drought treatments. Relatively high levels of nutrient availability increased the biomass of $B$. mollis, and consequently led to higher evapotranspiration rates and associated soil moisture depletion, thereby enhancing plant water stress after watering frequency was reduced, consistent with other studies (e.g., Zavaleta et al., 2003; Harpole et al., 2007). These results are in contrast to other studies, which showed that $\mathrm{N}$ deposition could improve plant water use efficiency and alleviate plant water stress by preventing cell membrane damage and enhancing osmoregulation (e.g., Van Schaik et al., 1997, Saneoka et al., 2004). 
Nutrient level and water co-limited photosynthesis and productivity of M. crystallinum and $B$. mollis (Figure 6.3; Figure 6.4), similar to other studies of grasslands across a large range of precipitation (Harpole et al., 2007, Eskelinen and Susan, 2015). Moreover, I found much lower leaf water potential (Figure 6.2), light availability (Supplementary Figure 6.3), aboveground plant N (Supplementary Figure 6.4), photosynthetic assimilation and total biomass (Figure 6.3; Figure 6.4) in M. crystallinum in mixture than alone; thus, B. mollis exerted a strong competitive effect on M. crystallinum for access to soil nutrients and light in high water conditions. This competition even outweighed the positive direct effects of increased nutrient availability on photosynthetic assimilation and total biomass of M. crystallinum (Figure 6.3; Figure 6.4) and the reduction in root/shoot ratio (Supplementary Figure 6.5). In fact, high-stature B. mollis took advantage of increased nutrient availability and constrained the growth of low-stature $M$. crystallinum by enhancement of shade effects (Yang et al., 2011, Sun et al., 2015). In agreement with other studies (Tilman, 1988, Lane et al., 2000, Harpole et al., 2007), M. crystallinum in response to light competition increased the biomass allocation to aboveground (Supplementary Figure 6.5), suggesting a shift in limiting resources from belowground (nutrients) to aboveground (light).

\subsubsection{CAM expression and reversibility in M. crystallinum as affected by competition}

Surprisingly, M. crystallinum did not switch from $\mathrm{C}_{3}$ photosynthesis to CAM expression over the entire experiment, a type of physiological plasticity $M$. crystallinum typically uses to adapt to environmental stress (Osmond, 1978, Winter and Holtum, 2007, 2014) and increase production of seeds and plant fitness (Cushman et al., 2008, Herrera, 2009). As discussed above, it is possible that light competition outweighed the effect of water competition on M. crystallinum in mixture with $B$. mollis. High frequency watering (once every two days) in high water conditions 
may alleviate the water stress of $M$. crystallinum even if it is competing with $B$. mollis. Other studies, however, indicated that even moderate water stress can induce CAM expression in $M$. crystallinum and that this effect increases with plant age (Winter and Holtum, 2007, 2014). Alternatively, in high water conditions photosynthesis and productivity of M. crystallinum in association with B. mollis was substantially suppressed (Figure 6.3; Figure 6.4) and thus did not have sufficient carbohydrates as substrates (Antony and Borland, 2008; Antony et al., 2008; Haider et al., 2012) to switch to CAM expression in response to water stress. Moreover, it was also observed that in high water conditions M. crystallinum (mixed with B. mollis) did not develop secondary leaves, a trait indicating the transition to adult stage in which CAM expression may be induced (Adams et al., 1998, Winter and Holtum, 2007). This is in contrast to the case of low water treatments (Figure 6.5), in which $M$. crystallinum reached the adult stage and was therefore capable of developing CAM expression. These results stress the importance of plant maturity in terms of sufficient carbohydrates instead of plant age in affecting CAM expression in $M$. crystallinum.

In summary, this study investigates how nutrient and water conditions affected the competitive relationship between invasive Mesembryanthemum crystallinum (a model facultative CAM species) and its interacting $\mathrm{C}_{3}$ species (Bromus mollis, also invasive) found in California's coastal grasslands. Our results showed that M. crystallinum outcompeted B. mollis under low water and high nutrient conditions, while $B$. mollis was a stronger competitor than $M$. crystallinum in access to light and soil nutrients in high water conditions. CAM expression in $M$. crystallinum was induced by moderate low water conditions with a reduction in rainfall frequency (i.e., once every 8 days), while reduction in nutrient availability applied in the whole stage of experiments did not upregulate CAM expression in M. crystallinum in these low water 
conditions. In response to intensive competition from B. mollis in access to soil nutrients and light resources (i.e., in high water conditions), M. crystallinum did not switch from $\mathrm{C}_{3}$ photosynthesis to CAM expression as an adaptive strategy. 


\title{
7 Hydraulic redistribution in tree-grass, CAM-grass, and tree-CAM associations: the implications of Crassulacean Acid Metabolism (CAM)
}

This chapter is adapted from Yu and Foster (2016), Modeled hydraulic redistribution in treegrass, CAM-grass, and tree-CAM associations: the implications of Crassulacean Acid Metabolism (CAM), Oecologia, 180:1113-1125 and Yu and D’Odorico, From facilitative to competitive interactions between woody plants and plants with Crassulacean Acid Metabolism (CAM): the role of hydraulic descent, Ecohydrology (In press).

\begin{abstract}
Past studies have largely focused on hydraulic redistribution (HR) in trees, shrubs, and grasses, and recognized its role in interspecies interactions. Hydraulic redistribution in plants that conduct crassulacean acid metabolism (CAM), however, remains poorly investigated, as does the effect of HR on transpiration in different vegetation associations (i.e., tree-grass, CAM-grass, and treeCAM associations). This study develops a mechanistic model to investigate the net direction and magnitude of hydraulic redistribution at the patch scale for tree-grass, CAM-grass, and tree-CAM associations at the growing season to yearly timescale. The modeling results show that deep-rooted CAM plants in CAM-grass associations could perform hydraulic lift at a higher rate than trees in tree-grass associations in a relatively wet environment, as explained by a significant increase in grass transpiration rate in the shallow soil layer, balancing a lower transpiration rate by CAM plants. CAM plants transpire during the night and thus perform hydraulic redistribution during the day. Based on these model simulations, the ability of CAM plants to perform hydraulic redistribution at a higher rate may have different effects on surrounding plant community than does plants with $\mathrm{C}_{3}$ or $\mathrm{C}_{4}$ photosynthetic pathways (i.e., diurnal transpiration).
\end{abstract}


By comparison, trees in tree-CAM associations may perform hydraulic descent at a higher rate than those in tree-grass associations in a dry environment. This high rate of hydraulic descent which increases with increase in investment of deep root can even turn the facilitation of CAM plants by woody plants into competition. Investment in deep roots by woody plants is usually thought to increase niche differentiation with shallow rooted plants, thereby reducing the competition and promoting species coexistence. This study indicates that deep root development could also favor competition through the mechanism of hydraulic descent, thereby changing my understanding of the role of root depth in niche differentiation between shallow and deep rooted plants.

\subsection{Introduction}

Deep-rooted plants that facilitate hydraulic redistribution (HR) can alter the availability of soil water resources in a water-limited plant community, thereby altering the community dynamics of the ecosystem. Hydraulic redistribution involves the passive translocation of water through roots either from wetter, deep soil to drier, shallow soil (hydraulic lift, HL) (Richards and Caldwell, 1987; Dawson, 1993; Ludwig et al., 2003) or from wetter, shallow soil to drier, deep soil (hydraulic descent, HD) (Burgess et al., 2001; Hultine et al., 2004). This process typically occurs at night when plant transpiration is negligible. During HR, water that is taken up by roots is released to the soil rather than being transported to the stem xylem (e.g., Ryel et al., 2002; Prieto et al., 2012). This process is found to occur in numerous ecosystems, including deserts, savannas, and temperate and tropical forests (Brooks, 2006; Neumann and Cardon, 2012; Prieto et al., 2012).

The ecohydrological benefits associated with HR include increased transpiration and photosynthesis (Lee et al., 2005; Scott et al., 2008), increased nutrient uptake (e.g., McCulley et al., 2004), and maintenance of root-soil contact and root hydraulic activity (Domec et al., 2004; 
Bauerle et al., 2008). This study focuses on the effects of HR on transpiration within plant communities. Water that is brought from the deep to the shallow layers can be used not only by plants that redistribute the water, but also by plants in the surrounding areas (Richards and Caldwell, 1987; Horton and Hart, 1998; Zou et al., 2005; Brooks et al., 2006) either directly via root uptake, or indirectly via ecto- and endomycorrhizal networks (CMNs) (Egerton-Warburton et al., 2007; Warren et al., 2008). In times of water stress, this phenomenon may prevent plants from reaching their wilting point. However, this use of the lifted water by the neighboring plants can also be seen as a loss of water from HR performing plants. Plants that facilitate HR are also able to redistribute it down to deep soil layers through hydraulic descent. Thus, after a rainfall event, they can move water to areas where other, non-HR plants cannot access it (Burgess et al., 2001; Hultine et al., 2004). This gives HR plants a competitive advantage over plant species that cannot facilitate HR (Horton and Hart, 1998). In a selection regime of reduced water loss, allocation toward aboveground biomass may be favored in arid environments, providing novel competitive potential.

Past studies have documented the occurrence of hydraulic redistribution (HR) in a variety of plant types that conduct $\mathrm{C}_{3}$ or $\mathrm{C}_{4}$ photosynthesis, including trees, shrubs, and grasses (Espeleta et al., 2004; Brooks et al., 2006; Baker et al., 2008), and recognized the role played by HR in interspecies interactions (Ludwig et al., 2004; Zou et al., 2005; Neumann and Cardon, 2012). The fact that these plants usually start transpiring (and thus taking up water) at the same time is the key factor in this sometimes competitive, sometimes facilitative relationship. However, what remains unclear is the effect of hydraulic redistribution by plants that conduct crassulacean acid metabolism (CAM). In contrast to $\mathrm{C}_{3}$ or $\mathrm{C}_{4}$ plants, $\mathrm{CAM}$ plants perform a temporal separation of the light and dark reactions of photosynthesis (Lüttge, 2004; Borland et al., 2011). Briefly, CAM plants open 
their stomata at night and fix $\mathrm{CO}_{2}$ into four-carbon acids, mediated by phosphoenolpyruvate (PEP) carboxylase. During the day, CAM plants close their stomata and decarboxylate the 4-C acids for $\mathrm{CO}_{2}$ refixation by $\mathrm{RuBisCO}$. The low water vapor concentration gradient at night leads to a lower transpiration rate and a higher water use efficiency by CAM plants (Lüttge, 2004; Ogburn and Edwards, 2010). Because plants that conduct CAM photosynthesis necessarily transpire at night, if a CAM plant were to facilitate hydraulic lift, its impact on the surrounding plant community may be different than that of an $\mathrm{HR}$ tree that conducts $\mathrm{C}_{3}$ photosynthesis.

Experimental evidence confirming the occurrence of HR by CAM plants is rare. This is presumably due to their shallow rooting systems (i.e., $\approx 5-20 \mathrm{~cm})($ e.g., Nobel, 2003; Ogburn and Edwards, 2010), which preclude the formation of a water potential gradient and thus the occurrence of HR (Caldwell et al., 1998; Espeleta et al., 2004). These shallow rooting systems may also have "rectifier" properties resulting from characteristics of the hypodermis and Casparian bands within the roots, thus preventing water release to drier soil (Nobel and Sanderson, 1984; North and Nobel, 1995; Borland et al., 2009; Ogburn and Edwards, 2010). This is in contrast to deep-rooted CAM plants, which have access to soil water resources at depth and thus may not develop roots with "rectifier" properties (Caldwell et al., 1998). Further, it has been shown that these barriers to outward flow do not halt the flow of water completely (Richards and Caldwell, 1987; Caldwell, 1998; Prieto et al., 2012). Yoder and Nowak (1999) documented the occurrence of hydraulic lift in a deep-rooted CAM species, Yucca schidigera, within the $350 \mathrm{~mm}$ root zone. Tree species such as Clusia hilariana and Clusia minor that also perform CAM photosynthesis (Borland et al., 2011) and have deep tap root systems may also be capable of performing HR. It was recently found that CAM species (i.e., Bromelia balansae) can facilitate tree seedlings on rocky outcrops of Atlantic forest, presumably because of their ability to perform hydraulic lift (Rocha et al., 2015). Thus, this 
study focuses on the ability of deep-rooted CAM plants to perform hydraulic redistribution (Table 7.1) and its effect on the surrounding plant community.

Table 7-1 A summary of CAM species which have some deep tap roots and may perform hydraulic redistribution

\begin{tabular}{|c|c|c|c|c|}
\hline Species name & Genus & Family & Description & Reference \\
\hline Yucca schidigera $^{1}$ & Yucca & Asparagaceae & tree-like; tall; deep tap roots & Yoder and Nowak (1999) \\
\hline Yucca baccata ${ }^{1}$ & Yucca & Asparagaceae & $\begin{array}{l}\text { closely related to Yucca } \\
\text { schidigera }\end{array}$ & Szarek and Troughton (1976) \\
\hline Carnegiea gigantea & Carnegiea & Cactaceae & $\begin{array}{l}\text { tree-like; tall; some tap } \\
\text { roots; large volume }\end{array}$ & $\begin{array}{l}\text { De la Barrera and Smith } \\
\text { (2009) }\end{array}$ \\
\hline Copiapoa atacamensis & Copiapoa & Cactaceae & $\begin{array}{l}\text { large tap roots; grow in the } \\
\text { Atacama Desert }\end{array}$ & Anderson (2001) \\
\hline Pereskia aculeata ${ }^{2}$ & Pereskia & Cactaceae & $\begin{array}{l}\text { facultative CAM ; tree-like; } \\
\text { tap roots }\end{array}$ & Rayder and Ting (1981) \\
\hline Portulacaria afra ${ }^{2}$ & Portulacaria & Portulacaceae & $\begin{array}{l}\text { facultative CAM; tree; tap } \\
\text { roots }\end{array}$ & $\begin{array}{l}\text { Ting (1981); Guralnick and } \\
\text { Ting (1987) }\end{array}$ \\
\hline $\begin{array}{l}\text { Clusia minor and Clusia } \\
\text { rosea }^{2}\end{array}$ & Clusiaceae & Clusiaceae & $\begin{array}{l}\text { facultative CAM; tree; deep } \\
\text { tap roots }\end{array}$ & $\begin{array}{l}\text { Herzog et al (1999); Winter } \\
\text { et al (2009); Borland et al } \\
(2011)\end{array}$ \\
\hline Clusia hilariana ${ }^{3}$ & Clusiaceae & Clusiaceae & $\begin{array}{l}\text { obligate CAM; tree; deep } \\
\text { tap roots }\end{array}$ & Miszalski et al (2013) \\
\hline
\end{tabular}

Note: ${ }^{1}$ Many other Yucca species with deep tap roots and tree-like characteristics include Yucca aloifolia, Yucca brevifolia, Yucca carnerosana, Yucca elata, Yucca filifera, et al.,

${ }^{2}$ Facultative CAM plants refer to those in which the degree of CAM expression depends on age and/or environmental cues.

${ }^{3}$ Obligate CAM refer to those which perform CAM independently of the environmental conditions (Winter et al., 2009; Borland et al., 2011). 
Because CAM plants close their stomata during the day (Lüttge, 2004), in CAM-grass associations, the total potential evapotranspiration (PET) during the daytime will be partitioned solely into grass PET and soil PET. This is expected to lead to more available water for grasses and a lower soil water potential in CAM-grass associations than would occur in tree-grass associations. Moreover, the low transpiration rate associated with CAM plants (Nobel et al., 1988; Lüttge, 2004; Ogburn and Edwards, 2010) is expected to reduce root uptake in the deep soil layer, thus increasing soil water potential in the deep soil layer. Therefore, I expect that CAM plants in CAM-grass associations will have a higher rate of hydraulic lift, as compared to trees in tree-grass associations. By comparison, trees in tree-CAM associations are expected to have a higher rate of hydraulic descent than trees in tree-grass associations, because of the lower transpiration rate of CAM plants (Nobel et al., 1988; Lüttge, 2004; Ogburn and Edwards, 2010) and a lack of grass transpiration in the shallow soil in tree-CAM associations. These expected interactions between different plant functional groups (including CAM species) in systems with plants performing HR have never been explored in detail. Despite the recognized importance of HR as a modulator of plant access to soil water, a process-based theory of its impact on community dynamics is still missing.

Models have been widely used and proved to be valuable tools to investigate the role of hydraulic redistribution in ecosystems dominated by $\mathrm{C}_{3}$ and/or $\mathrm{C}_{4}$ species at different spatial and temporal scales (e.g., Lee et al., 2005; Neumann and Cardon, 2012). In this study, I develop a mechanistic model to investigate these expected interactions and the net direction and magnitude of hydraulic redistribution at the patch scale for tree-grass, CAM-grass, and tree-CAM associations at the growing season to yearly timescales (Table 7.2). I also evaluate the effects of 
hydraulic redistribution on transpiration at the patch scale for these three types of vegetation associations. Using this model, I aim to clarify the key processes controlling hydraulic redistribution in these three types of vegetation associations and the implications for interspecies interactions.

Table 7-2 A summary of vegetation associations in this study

\begin{tabular}{lllll}
\hline $\begin{array}{l}\text { Vegetation } \\
\text { associations }\end{array}$ & Deep rooted plants & $\begin{array}{l}\text { Shallow } \\
\text { rooted plants }\end{array}$ & $\begin{array}{l}\text { Plants performing } \\
\text { HR at night }\end{array}$ & $\begin{array}{l}\text { Plants performing } \\
\text { HR in the daytime }\end{array}$ \\
\hline Tree-Grass & Tree & Grass & Tree & CAM \\
CAM-Grass & CAM & Grass & & \\
Tree-CAM & Tree & CAM & Tree & \\
\hline
\end{tabular}

7.2 Materials and methods

\subsubsection{Model development}

I develop a mechanistic model to quantify the net direction and magnitude of hydraulic redistribution (HR) at the patch scale for tree-grass, CAM-grass, and tree-CAM associations at the growing season to yearly timescale (Table 7.2). The model accounts for soil moisture dynamics of two soil layers coupled by hydraulic redistribution by deep-rooted species (Ryel et al., 2002; Lee et al., 2005; Yu and D’Odorico, 2014b; see the section on soil moisture dynamics for details). In this study, I focus on situations where deep soil moisture is supplied by surface soil water drainage (Yu and D'Odorico, 2014b; Yu and D'Odorico, 2015a b), in contrast to other studies in which groundwater uptake from a shallow water table is considered (e.g., Mooney et al., 1880; Ryel et al., 2002; Hultine et al., 2004). My model evaluates the effects of hydraulic redistribution on transpiration at the patch scale for these three types of vegetation associations. 
CAM plants transpire at night (12 hours), and deep-rooted CAM plants are assumed to perform HR in the daytime (12 hours) when stomata are closed (Lüttge, 2004). In contrast, trees transpire in the daytime (12 hours) and perform HR at night (12 hours) (Ryel et al., 2002; Prieto et al., 2012). CAM plants are often succulents with a non-negligible plant water capacitance (Lüttge, 2004; Ogburn and Edwards, 2010). Following other studies (Lhomme et al., 2001; Bartlett et al., 2014), I account for the plant water capacitance by modeling the transient water storage dynamics. In contrast, root uptake by trees and grasses is calculated at a steady state, neglecting the effects of plant water capacitance (Manzoni et al., 2013). The soil moisture dynamics are simulated in two soil types (sandy loam and loamy sand) and two rainfall regimes (dry and relatively wet) at the time scale of a half-hour. As CAM plants predominantly occur in drylands, low root hydraulic conductivity resulting from fine root death, root embolism, or the loss of soilroot contact (Caldwell et al., 1998; Domec et al., 2004; Espeleta et al., 2004) may limit the occurrence of HR. To this purpose, two gradients of root hydraulic conductivity by CAM plants (high, $C H$; and low, $C L$ ) are used to investigate its effect on the rate of hydraulic redistribution. Another typical characteristic for CAM plants is their relatively low mesophyll conductance, which results from water storage and thus restricted gas space for the diffusion of $\mathrm{CO}_{2}$ into the photosynthetic tissue (e.g., Flexas et al., 2008; Nelson and Sage, 2008; Ripley et al., 2013). To this end, two gradients of mesophyll conductance by CAM plants (high and low) are used to investigate its effect on the rate of hydraulic redistribution.

\subsubsection{Soil moisture dynamics}

Soil moisture dynamics in the two soil layers are modeled by two coupled equations

$$
n Z_{1} \frac{d s_{1}}{d t}=P-U_{1}-E-D_{1}+H R
$$


and

$$
n Z_{2} \frac{d s_{2}}{d t}=D_{1}-U_{2}-D_{2}-H R
$$

where the subscripts 1 and 2 refer to the shallow and deep soil layer, respectively; $\mathrm{n}$ is the soil porosity, $Z_{1}$ and $Z_{2}$ the soil layer thickness $(\mathrm{mm}), S_{1}$ and $S_{2}$ the relative soil moisture $\left(0<S_{1}\right.$, $\left.S_{2} \leq 1\right), P$ the rate of rainfall infiltration into the top soil layer $\left(\mathrm{mm} \mathrm{d}^{-1}\right), U_{1}$ and $U_{2}$ the soil moisture losses from each soil layer due to root uptake $\left(\mathrm{mm} \mathrm{d}^{-1}\right), E$ the evaporation rate from the soil surface $\left(\mathrm{mm} \mathrm{d}^{-1}\right), D_{1}$ and $D_{2}$ the drainage rates $\left(\mathrm{mm} \mathrm{d}^{-1}\right)$, and $H R$ the hydraulic redistribution at the patch scale $\left(\mathrm{mm} \mathrm{d}^{-1}\right)$. Positive values of $H R$ indicate "hydraulic lift" (i.e., upward hydraulic redistribution), while negative values of $H R$ indicate "hydraulic descent" (i.e., downward hydraulic redistribution).

Precipitation is modeled as a sequence of intermittent rainfall events occurring as a marked Poisson process with average rainfall frequency, $\lambda$, (events per day). The depth (mm) of each storm is modeled as an exponentially distributed random variable with mean, $h$, (mm per event) (Rodriguez-Iturbe et al., 1999a). Runoff occurs when the surface layer is saturated (i.e., $S_{1}=1$ ). Drainage is assumed to be driven only by gravity and is expressed as $D=\frac{K_{s} \exp \left(\beta\left(S-S_{f c}\right)-1\right)}{\exp \left(\beta\left(1-S_{f c}\right)-1\right)}$, where $K_{S}$ is the soil saturated hydraulic conductivity $\left(\mathrm{mm} \mathrm{h}^{-1}\right), \beta$ a coefficient, $S$ the relative soil moisture, $S_{f c}$ is the field capacity (Laio et al., 2001).

Hydraulic redistribution is determined as $\mathrm{HR}=c C_{r \max }\left(\Psi_{s 2}-\Psi_{s 1}\right) \min \left(r_{1}, r_{2}\right)$ (Ryel et al., 2002; Lee et al., 2005; Yu and D’Odorico, 2014b), where $C_{r m a x}$ is the maximum root hydraulic conductance of the entire active root system $\left(\mathrm{mm} \mathrm{MPa}^{-1} \mathrm{~h}^{-1}\right), c$ a factor reducing root hydraulic conductance and accounting for soil water limitation (Supplementary Table 7.1), $\Psi_{S 2}$ and $\Psi_{S 1}$ the 
soil water potential (MPa) in the deep and the shallow soil layer, respectively. Following Clapp and Hornberger (1978), $\Psi$ is determined as $\Psi=\Psi_{S} \times S^{-d}$, where $\Psi$ is soil water potential, $S$ soil moisture, while $\Psi_{S}$ and $d$ are experimentally derived parameters that have been determined for a variety of soils.

\subsubsection{Root uptake by trees and grasses}

The effects of plant water capacitance by trees and grasses are thought to be insignificant and thus their root uptake is calculated at steady state (Manzoni et al., 2013). The maximum total potential evapotranspiration in the daytime $\left(E T_{\operatorname{maxd}}\right)$ and at night $\left(E_{\operatorname{maxn}}\right)$ is assumed to be constant $\left(E T_{\text {maxd }}=4.5 \mathrm{~mm} \mathrm{~d}^{-1}\right.$ and $\left.E_{\text {maxn }}=0.5 \mathrm{~mm} \mathrm{~d}^{-1}\right)($ Porporato et al., 2003). Note that $E_{\text {maxn }}$ is used to determine only the soil evaporation at night but not transpiration by CAM plants. $E T_{\text {maxd }}$ is then partitioned among different plant functional groups as follows. In the case of tree-grass associations, $E T_{\operatorname{maxd}}$ is partitioned into potential transpiration for trees $\left(T_{\text {tmaxd }}\right)$, grasses growing under the tree canopy $\left(T_{\text {gmaxd }}\right)$, and evaporation for soil surface $\left(E_{\operatorname{maxd}}\right)$, respectively. Potential evapotranspiration depends on shortwave radiation available to overstory and understory canopies, whereas shortwave radiation exponentially decays through the plant canopy (Beer's law). Thus, following Caylor et al (2005) and Yu and D’Odorico (2014 a b), $T_{\text {tmaxd }}$ is determined as $T_{\text {tmaxd }}=E T_{\text {maxd }}\left[1-\exp \left(-k_{s} L A I_{t}\right)\right]$, where $k_{s}$ is the extinction coefficient of shortwave radiation, $L A I_{t}$ the tree leaf area index $\left(\mathrm{m}^{2} \mathrm{~m}^{-2}\right)$. Accordingly, I have $T_{\text {gmaxd }}+E_{\text {maxd }}=E T_{\text {maxd }} \exp \left(-k_{s} L A I_{t}\right)$, where $T_{\text {gmaxd }}$ is assumed to depend on grass cover $\left(f_{g}\right)$, given by $T_{\text {gmaxd }}=E T_{\text {maxd }} \exp \left(-k_{s} L A I_{t}\right) f_{g}$. In the case of CAM-grass associations, due to the lack of transpiration by CAM plants during the day, $E T_{\text {maxd }}$ is partitioned into potential transpiration for grasses $\left(T_{\text {gmaxd }}\right)$ and evaporation for soil surface $\left(E_{\operatorname{maxd}}\right)$. Again, $T_{\text {gmaxd }}$ is 
assumed to depend on grass cover $\left(f_{g}\right)$ and is determined as $T_{\text {gmaxd }}=E T_{\text {maxd }} f_{g}$. In the case of tree-CAM associations, $E T_{\operatorname{maxd}}$ is partitioned into potential transpiration for trees $\left(T_{\text {tmaxd }}\right)$ and evaporation for soil surface $\left(E_{\operatorname{maxd}}\right)$. Accordingly, I have $T_{t \operatorname{maxd}}=E T_{\operatorname{maxd}}\left[1-\exp \left(-k_{s} L A I_{t}\right)\right]$ and $E_{\operatorname{maxd}}=E T_{\operatorname{maxd}} \exp \left(-k_{s} L A I_{t}\right)$.

Potential transpiration $\left(T_{d \max }\right)$ for deep-rooted plants is then partitioned into potential transpiration for the shallow soil layer $\left(T_{1 d \max }\right)$ and the deep soil layer $\left(T_{2 d \max }\right)$, respectively. Following Yu and D'Odorico (2014 a), $T_{1 \text { dmax }}$ and $T_{2 d \max }$ are determined as $T_{1 \text { dmax }}=$ $T_{d \max } Z_{1} S_{1} /\left(Z_{1} S_{1}+Z_{2} S_{2}\right)$ and $T_{2 d \max }=T_{d \max } Z_{2} S_{2} /\left(Z_{1} S_{1}+Z_{2} S_{2}\right)$. The actual transpiration by plants is then determined by accounting for the soil water availability. According to Rodriguez-Iturbe et al (1999), I have

$$
\tau(\mathrm{S})=\left\{\begin{array}{lc}
0, & S<S_{w} \\
\frac{S-S_{w}}{S^{*}-S_{w}}, & \text { and } S<S^{*} \\
1, & \text { and } S \geq S^{*}
\end{array}\right.
$$

where $\tau(\mathrm{S})$ is limitation coefficient of soil moisture, $S$ the soil moisture, $S^{*}$ the vegetationspecific value of relative soil moisture above which transpiration is not limited by soil water availability, and $S_{w}$ the vegetation-specific wilting point at which transpiration ceases. For simplicity, trees and grasses are assumed to have the same values of $S_{w}$ and $S^{*}$ (Caylor et al., 2006; Yu and D'Odorico, 2014a). Thus, the actual transpiration for shallow rooted plants $\left(T_{1 s}\right)$ is calculated as $T_{1 s}=T_{1 \text { smax }} \tau\left(S_{1}\right)$, where $T_{1 \text { smax }}$ is potential transpiration for shallow rooted plants. The actual deep-rooted transpiration is contributed by root uptake from the shallow $\left(T_{1 d}\right)$ and the deep $\left(T_{2}\right)$ soil layers, which depend on the root profile as $T_{1 d}=T_{1 d \max } \tau\left(S_{1}\right) r_{1}$ and $T_{2}=$ $T_{2 d \max } \tau\left(S_{2}\right) r_{2}$, where $r_{1}$ and $r_{2}$ are cumulated (and normalized) tree root densities in the shallow and the deep soil layers, respectively $\left(r_{1}+r_{2}=1\right)$. 


\subsubsection{Root uptake by CAM plants}

The model of root uptake by CAM plants used in this study follows the framework by Bartlett et al (2014). A detailed description of the model can be found in Bartlett et al (2014). The model incorporates capacitances and resistances into the water flow pathway using an analogy with electric circuits (Figure 7.1).

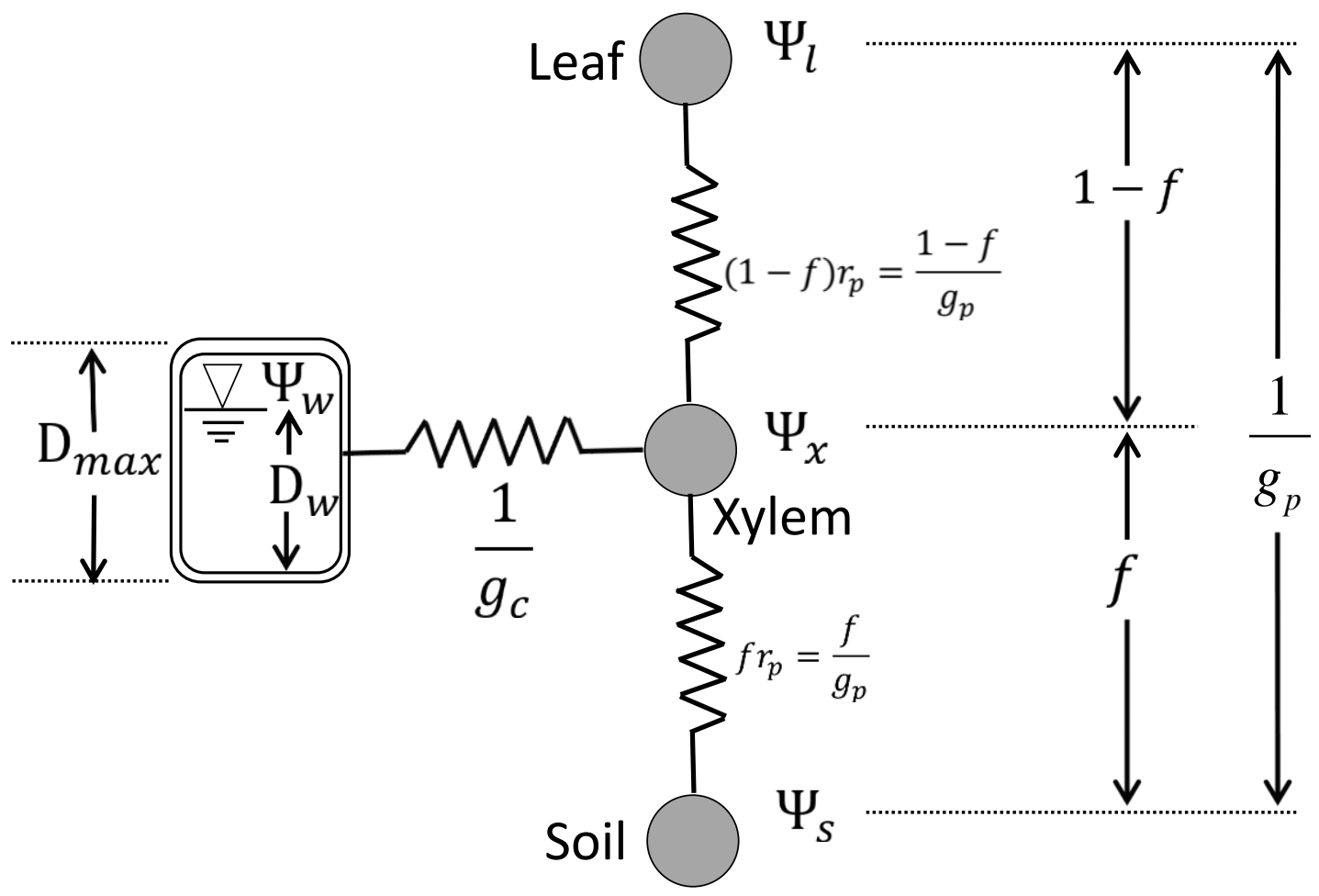

Figure 7-1: Schematic diagram of water flux within canopies of CAM plants. $\Psi_{1}$ : leaf water potential; $\Psi$ s: soil water potential; $\Psi \mathrm{x}$ : xylem water potential; $\Psi \mathrm{w}$ : plant storage water potential; f: fraction of plant resistance below the storage branch connection; $\mathrm{g}_{\mathrm{p}}$ plant conductance per unit leaf area $\left(r_{p}\right.$, plant resistance per unit leaf area); $g_{c}$ storage conductance per unit leaf area; $D_{\max }$ maximum depth of water per unit leaf area; $D_{w}$ actual depth of water per unit leaf area. Adapted from Lhomme et al (2001) and Bartlett et al (2014).

A parameter $f$ represents the fraction of plant resistance below the storage branch connection and is taken to be 0.5 as in Bartlett et al (2014). The leaf transpiration $\left(T_{C A M}\right)$ per unit ground 
area is balanced by the rates of root uptake from the two soil layers, $U_{1 C A M}$ and $U_{2 C A M}$, respectively, and the plant water capacitance $\left(Q_{w}\right)$, i.e.,

$$
T_{C A M}=U_{1 C A M}+U_{2 C A M}+Q_{w},(3)
$$

In this mass balance equation, both $U_{1 C A M}$ and $U_{2 C A M}$ and $Q_{w}$ are controlled by water potential gradients, i.e., $U_{1 C A M}=g_{s r p 1}\left(\Psi_{s 1}-\Psi_{x}\right) r_{1}, U_{2 C A M}=g_{s r p 2}\left(\Psi_{s 2}-\Psi_{x}\right) r_{2}$, and $Q_{w}=$ $g_{c} L A I_{c}\left(\Psi_{w}-\Psi_{x}\right)$, respectively, where $g_{s r p 1}$ is the soil-root-plant conductance per unit ground area for the shallow soil layer $\left(\mathrm{m} \mathrm{s}^{-1} \mathrm{MPa}^{-1}\right), g_{\text {srp } 2}$ the soil-root-plant conductance per unit ground area for the deep soil layer $\left(\mathrm{m} \mathrm{s}^{-1} \mathrm{MPa}^{-1}\right), g_{c} L A I_{c}$ the storage conductance per unit ground area $\left(\mathrm{m} \mathrm{s}^{-1} \mathrm{MPa}^{-1}\right)\left(g_{c}\right.$ is the storage conductance per unit leaf area), $\Psi_{x}$ the water potential at the storage connection (MPa), $\Psi_{w}$ the plant storage water potential (MPa). Note that the components in equation 4 are expressed per unit ground area. Negative values of $Q_{w}$ indicate that $\Psi_{x}$ is greater than $\Psi_{w}$ and thus root uptake recharges the plant capacitance. The readers are refer to Yu and Foster (2016) for details of calculations of $g_{s r p 1}, g_{s r p 2}$, and $\Psi_{w} \cdot T_{C A M}$ is also equal to the flux flowing from the storage connection to the leaves, i.e.,

$$
T_{C A M}=\frac{g_{p} L A I_{c}}{1-f}\left(\Psi_{x}-\Psi_{l}\right)(4)
$$

where $g_{p}$ is plant conductance per unit leaf area and $\frac{g_{p} L A I_{c}}{1-f}$ represents the plant conductance per unit ground area between the storage connection node potential $\left(\Psi_{x}\right)$ and leaf water potential $\left(\Psi_{l}\right.$, $\mathrm{MPa})$.

The leaf transpiration rate $\left(T_{C A M}\right)$ per unit ground area is also driven by the specific humidity gradient between the leaf mesophyll $\left(q_{l}\right)$ and the atmosphere $\left(q_{a}\right)$, i.e., 


$$
T_{C A M}=l g_{m s a} \frac{\rho_{a}}{\rho_{w}}\left(q_{l}-q_{a}\right),(5)
$$

where $\rho_{a}$ is the density of air $\left(\mathrm{Kg} \mathrm{m}^{-3}\right), \rho_{w}$ the density of water $\left(\mathrm{Kg} \mathrm{m}^{-3}\right), g_{m s a}$ the series of the mesophyll, stomatal, and atmospheric conductances $\left(\mathrm{m} \mathrm{s}^{-1}\right)$ to water vapor per unit ground at well watered conditions, i.e., $g_{m} L A I_{c}, g_{s} L A I_{c}$, and $g_{a}$, respectively, i.e., $g_{m s a}=$

$L A I_{c} g_{m} g_{s} \frac{g_{a}}{L A I_{c} g_{m} g_{s}+g_{s} g_{a}+g_{m} g_{a}}, l$ a coefficient limiting $g_{m s a}$ at dry conditions calculated as Bartlett et al (2014). $g_{a}$ is taken to be constant (Bartlett et al., 2014); $g_{s}$, in turn, is calculated as $g_{s}=1.6 g_{s C}$, where $g_{s C}$ is the stomatal conductance for $\mathrm{CO}_{2}$ and is taken to be constant (Supplementary Table 7.1). In equation (5), $q_{a}$ depends on air temperature ( $T_{a}$, Kelvin) and relative humidity $(R H, \%)$ (Supplementary Table 7.1$) ; q_{l}$ is a function of leaf water potential $\left(\Psi_{l}\right)$ and leaf temperature. The readers are refer to Yu and Foster (2016) for detailed calculations of $q_{l}$. The rate of CAM plant uptake is calculated combing equations (3)-(5) as in Bartlett et al (2014) with equation (5) driven by atmospheric conditions.

\subsubsection{Soil evaporation}

Following Porporato et al (2003) and Bartlett et al (2014), evaporation (E) at the soil surface is represented by a linear dependence with the relative soil moisture, given by

$$
E= \begin{cases}0, & 0 \leq S \leq S_{h} \\ E_{\max } \frac{S-S_{h}}{1-S_{h}}, & S_{h}<S<1\end{cases}
$$

where $S_{h}$ is the hygroscopic point below which evaporation at the soil surface cease (Laio et al., 2001) and $E_{\max }$ the potential evaporation in the day time or at night. Calculations of $E_{\max }$ can be found in section 2.2.2. 


\subsubsection{Parameterization of the model}

I parameterize the model with respect to environmental conditions with low $\left(\lambda=0.1 \mathrm{~d}^{-1}\right.$ and $h=$ $10 \mathrm{~mm})$ and moderate $\left(\lambda=0.2 \mathrm{~d}^{-1}\right.$ and $\left.h=10 \mathrm{~mm}\right)$ total rainfall amount, in a way that $\mathrm{I}$ can compare HR at the patch scale in tree-grass associations with that in CAM-grass and tree-CAM associations. Because this study focuses on hydraulic redistribution in these three vegetation associations and the effect of HR on interspecies interactions, the growing seasons of trees, grasses and CAM plants are assumed to coincide and last 210 days each year (Bhattachan et al., 2012). Root depths of deep-rooted plants and shallow-rooted plants are taken to be $40 \mathrm{~cm}$ and 20 cm, respectively (Yoder and Nowak, 1999; Nippert et al., 2012; Yu and D’Odorico, 2014b). The value of the root depth of deep-rooted plants $(40 \mathrm{~cm})$ is smaller than that in other studies (e.g., Mooney et al., 1980; Ryel et al., 2002; Hultine et al., 2004) because deep-rooted CAM plants typically have relatively "shallow" roots (Yoder and Nowak, 1999; Lüttge, 2004). An increase in the root depth of deep-rooted plants reduces the hydraulic lift in relatively wet environments $(\mathrm{Yu}$ and D'Odorico, 2014b) but does not change the general pattern shown in this study. Two gradients of root hydraulic conductivity by CAM plants (high, $C H$ and low, $C L$ ) are taken to be $0.75 L^{2} I_{t} \mathrm{~mm} \mathrm{MPa}^{-1} \mathrm{~h}^{-1}$ and $0.4 L A I_{t} \mathrm{~mm} \mathrm{MPa}^{-1} \mathrm{~h}^{-1}$, respectively. I consider two values of mesophyll conductance: a higher value of $0.003 \mathrm{~m} \mathrm{~s}^{-1}$ (Bartlett et al., 2014) and a lower value of $0.0015 \mathrm{~m} \mathrm{~s}^{-1}$. The cumulative (and normalized) tree root densities in the shallow $\left(r_{1}\right)$ and the deep soil layers $\left(r_{2}\right)$ are taken to be 0.4 and 0.6 , respectively in dry environment $\left(\lambda=0.1 \mathrm{~d}^{-1}\right.$ and $h=10 \mathrm{~mm}$ ) because in these conditions a significant hydraulic descent occurs ( $\mathrm{Yu}$ and D'Odorico, 2014b). I also consider the case with $r_{1}=0.7$ and $r_{2}=0.3$ in relatively wet environment $\left(\lambda=0.2 \mathrm{~d}^{-1}\right.$ and $\left.h=10 \mathrm{~mm}\right)$ with the occurrence of hydraulic lift (Yu and D'Odorico, 2014b). The values of grass cover $\left(f_{g}\right)$ in tree-grass and CAM-grass associations in 
the dry and relatively wet environments are taken to be $80 \%$ and $100 \%$, respectively; other values of $f_{g}$ do not change the general pattern shown in this study. Leaf area index for trees $\left(L A I_{t}\right)$ in tree-grass and tree-CAM associations, and CAM plants $\left(L A I_{C}\right)$ in CAM-grass associations in the dry and relatively wet environments are taken to be $1.5 \mathrm{~m}^{2} \mathrm{~m}^{-2}$ and $3 \mathrm{~m}^{2} \mathrm{~m}^{-2}$, respectively, while $1 \mathrm{~m}^{2} \mathrm{~m}^{-2}$ and $2 \mathrm{~m}^{2} \mathrm{~m}^{-2}$ for CAM plants $\left(L A I_{c}\right)$ in tree-CAM associations. Two soil types used in this study are sandy loam and loamy sand. Parameters describing various soil characteristics required in this study can be found in Laio et al (2001) and Yu and D'Odorico (2014a). The values of all the other parameters used in this study are presented in Supplementary Table 7.1.

\subsection{Results}

The modeling results show that in a dry environment, the CAM-grass associations $(\mathrm{CH}-\mathrm{G}$ and CL-G) reduce the magnitude of hydraulic descent while the tree-CAM associations (T-C) increase the magnitude of hydraulic descent, as compared with tree-grass associations (T-G). For example, hydraulic descent in sandy loam and loamy sand averages $-0.12 \mathrm{~mm} \mathrm{~d}^{-1}$ and $-0.09 \mathrm{~mm}$ $\mathrm{d}^{-1}$ in the tree-grass associations, while $-0.07 \mathrm{~mm} \mathrm{~d}^{-1}$ and $-0.04 \mathrm{~mm} \mathrm{~d}^{-1},-0.14 \mathrm{~mm} \mathrm{~d}^{-1}$ and -0.12 $\mathrm{mm} \mathrm{d}^{-1}$ in CAM-grass associations $(\mathrm{CH}-\mathrm{G})$ and tree-CAM associations, respectively (Figure 7.2a). In a relatively wet environment, the CAM-grass associations ( $\mathrm{CH}-\mathrm{G}$ and $\mathrm{CL}-\mathrm{G})$ increases the magnitude of hydraulic lift while the tree-CAM associations (T-C) reduces the magnitude of hydraulic lift, as compared with the tree-grass association (T-G) (Figure 7.2b). 

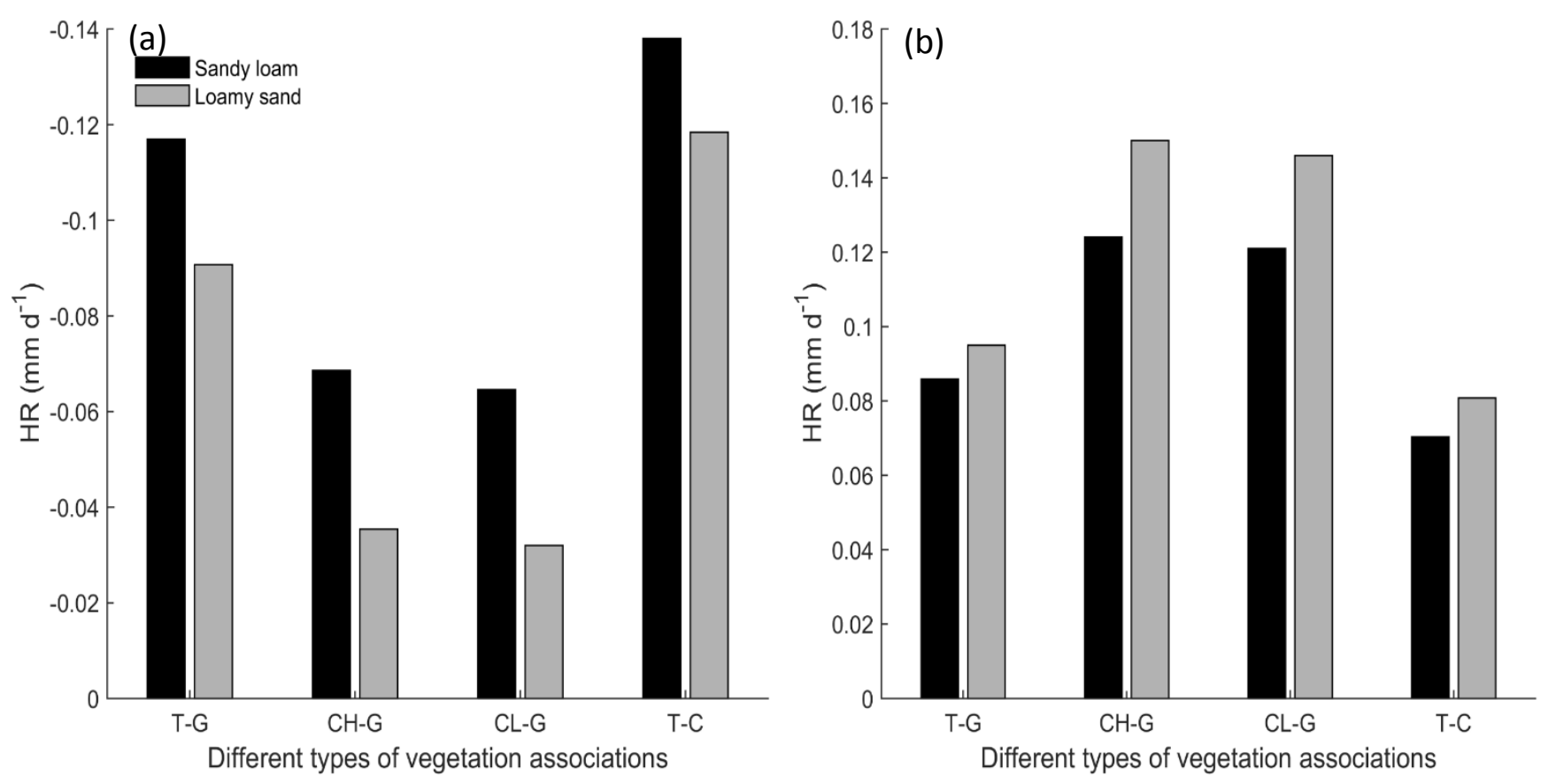

Figure 7-2: Hydraulic redistribution (HR) as affected by different types of vegetation associations with $\mathrm{h}=10 \mathrm{~mm}, \lambda=0.1 \mathrm{~d}^{-1}$ (a) and $\lambda=0.2 \mathrm{~d}^{-1}$ (b) for the case of a high mesophyll conductance $\left(\mathrm{g}_{\mathrm{m}}=0.003 \mathrm{~m} \mathrm{~s}^{-1}\right)$. T-G refers to Tree-Grass association; $\mathrm{CH}-\mathrm{G}$ refers to CAM-Grass association with a relatively high value of hydraulic conductivity; CL-G refers to CAM-Grass association with a relatively low value of hydraulic conductivity; T-C refers to Tree-CAM association.

These patterns predicted by the model (differences of hydraulic redistribution in these three vegetation associations) are more evident with CAM plants with lower mesophyll conductance (Figure 7.2a, b; Supplementary Figure 7.1a, b). For instance, in a relatively wet environment, the increase in hydraulic lift in the CAM-grass associations ( $\mathrm{CH}-\mathrm{G}$ and $\mathrm{CL}-\mathrm{G})$ relative to the treegrass association (T-G) is higher with CAM plants with a lower mesophyll conductance (Figure 7.2a, b; Supplementary Figure 7.1a, b). Interestingly, a relatively low value of hydraulic conductivity by CAM plants does not significantly limit HR in the long time scale in both dry and relatively wet environments (Figure 7.2a, b; Supplementary Figure 7.1a, b). An increase in soil hydraulic conductivity from sandy loam to loamy sand reduces the magnitude of hydraulic 
descent in the dry environment (Figure 7.2a; Supplementary Figure 7.1a), while increasing the magnitude of hydraulic lift in a relatively wet environment for all the three vegetation associations (Figure 7.2b; Supplementary Figure 7.1b).

The modeling results also show that CAM plants have a lower transpiration rate in both dry (Figure 7.3a) and relatively wet (Figure 7.3b) environments, as seen from a comparison of the tree-grass associations, CAM-grass associations, and tree-CAM associations; in fact, the actual transpiration rate by CAM plants in the CAM-grass associations $(\mathrm{CH}-\mathrm{G})$ is $0.16 \mathrm{~mm} \mathrm{~d}^{-1}$ and 0.54 $\mathrm{mm} \mathrm{d}^{-1}$ in dry (Figure 7.3a) and relatively wet (Figure 7.3b) environments, respectively, which is about half of the values for trees in tree-grass associations. The lower transpiration rate by CAM plants $\left(T_{1 d}+T_{2}\right)$ leads to a higher transpiration rate of grasses in the CAM-grass associations $\left(T_{1 s}\right)$ than that in tree-grass associations in both dry (Figure 7.3a) and relatively wet (Figure 7.3b) environments. Likewise, the lower transpiration rate by CAM plants $\left(T_{1 s}\right)$ and a lack of grass coverage in tree-CAM associations leads to a higher rate of evaporation at the soil surface $(E)$ than of the tree-grass and CAM-grass associations in both dry (Figure 7.3a) and relatively wet (Figure 7.3b) environments. The ratio of actual evapotranspiration contributed by the shallow soil layer $\left(E T_{1}=T_{1 d}+T_{1 s}+E\right)$ to transpiration $\left(T_{2}\right)$ contributed by the deep soil layer is greater in the CAM-grass associations than of the tree-grass and CAM-grass associations in both dry (Figure 7.3c) and relatively wet (Figure 7.3d) environments. 

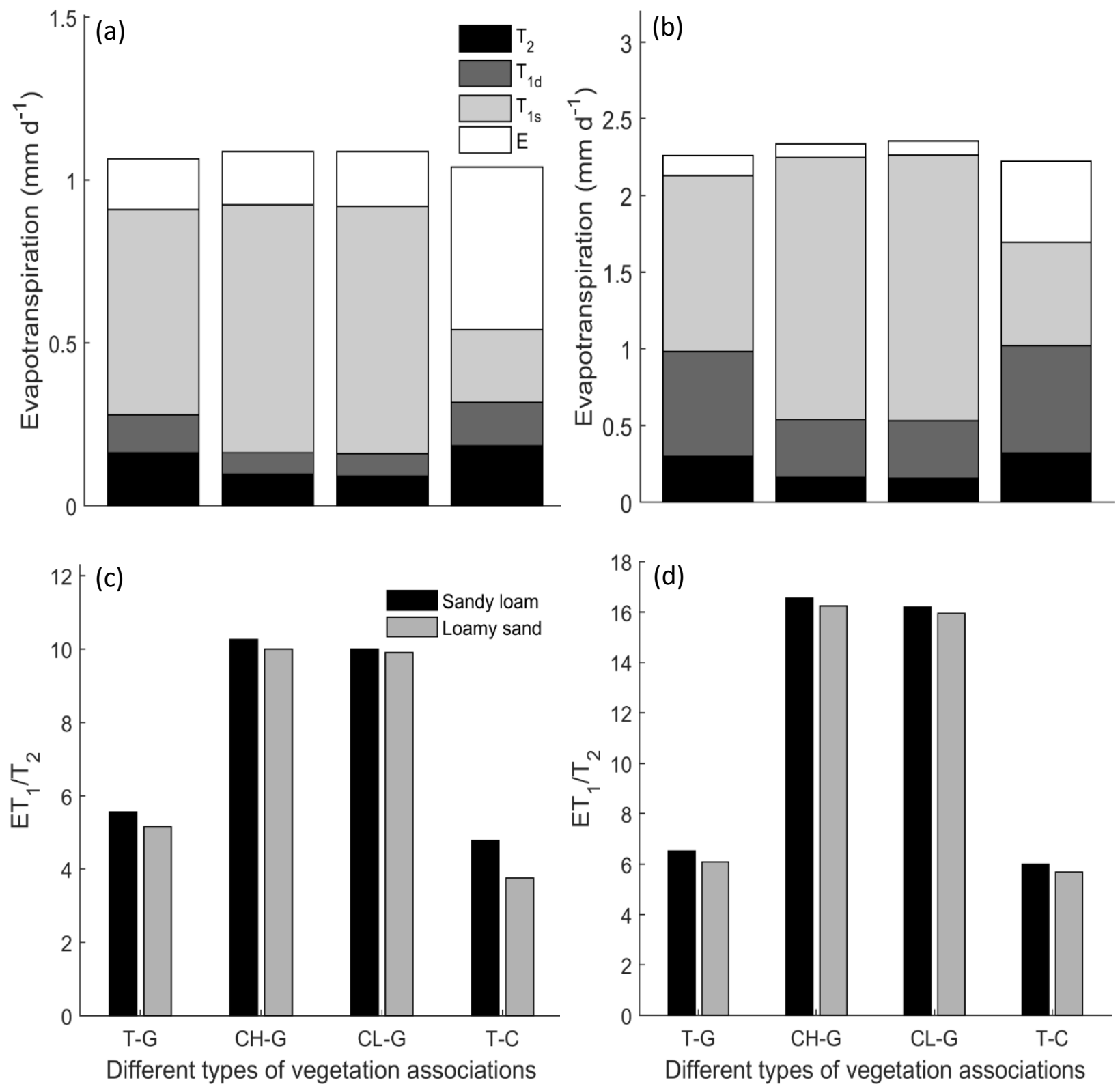

Figure 7-3: $(a, b)$ Actual evapotranspiration components as affected by different types of vegetation associations with $h=10 \mathrm{~mm}, \lambda=0.1 \mathrm{~d}^{-1}$ (a) and $\lambda=0.2 \mathrm{~d}^{-1}(\mathrm{~b})$ for the case of a high mesophyll conductance $\left(\mathrm{g}_{\mathrm{m}}=0.003 \mathrm{~m} \mathrm{~s}^{-1}\right)$. $\mathrm{T}_{2}$ refers to transpiration in the deep soil layer; $\mathrm{T}_{1 \mathrm{~d}}$ refers to transpiration in the shallow soil layer by deep-rooted plants; $\mathrm{T}_{1 \mathrm{~s}}$ refers to transpiration in the shallow soil layer by shallow-rooted plants; E refers to evaporation at soil surface. (c, d) The ratio of actual evapotranspiration in the shallow soil layer $\left(\mathrm{ET}_{1}=\mathrm{T}_{1 \mathrm{~d}}+\mathrm{T}_{1 \mathrm{~s}}+\mathrm{E}\right)$ to transpiration in the deep soil layer $\left(\mathrm{T}_{2}\right)$ as affected by different types of vegetation associations with $\mathrm{h}=10$ $\mathrm{mm}, \lambda=0.1 \mathrm{~d}^{-1}(\mathrm{c})$ and $\lambda=0.2 \mathrm{~d}^{-1}(\mathrm{~d})$. 
Hydraulic descent increases the transpiration rate by deep-rooted plants $\left(T_{d}\right)$ (Supplementary Figure 7.2a) and reduces the transpiration rate by shallow-rooted plants $\left(T_{s}\right)$ (Supplementary Figure 7.2b) in the dry environment. In contrast, hydraulic lift reduces the transpiration rate by deep -rooted plants (Supplementary Figure 7.2c) and increases the transpiration rate by shallowrooted plants (Supplementary Figure 7.2d) in relatively wet environments. Higher values of HR correspond to a higher difference of transpiration with and without HR (Supplementary Figure 7.2).

\subsection{Discussion}

Hydraulic redistribution is an important modulator of plant access to soil water, a crucial resource in arid environments. Studies of hydraulic redistribution continue to engender scientific interest because of its wide occurrence within a range of different ecosystems and plant species (Brooks, 2006; Neumann and Cardon, 2012; Prieto et al., 2012) and its ecohydrological benefits (McCulley et al., 2004; Lee et al., 2005; Bauerle et al., 2008; Scott et al., 2008). Past studies have largely focused on hydraulic redistribution by plants with $\mathrm{C}_{3}$ or $\mathrm{C}_{4}$ photosynthetic pathways at different spatial and temporal scales (Espeleta et al., 2004; Brooks et al., 2006; Baker et al., 2008; Neumann and Cardon, 2012), while the ability of CAM plants to perform hydraulic redistribution has been ignored (but see Yoder and Nowak, 1999). This study develops a mechanistic model to quantify the net direction and magnitude of hydraulic redistribution (HR) at the patch scale for tree-grass, CAM-grass, and tree-CAM associations at the growing season to yearly timescale. By clarifying the key processes/factors determining differences in HR in these three vegetation associations, these model simulations provide the theoretical basis for the mechanisms underlying plant access to soil water and the implications for interspecies interactions. 
The modeling results show that deep-rooted CAM plants in CAM-grass associations may perform a higher rate of hydraulic lift than trees in tree-grass associations in relatively wet environments $\left(\lambda=0.2 \mathrm{~d}^{-1}\right.$ and $h=10 \mathrm{~mm}$ ) (Figure 7.2b). This result can be explained by a higher rate of transpiration rate of grasses (balancing a low transpiration rate of CAM plants), and thus a lower water potential in the shallow soil layer in CAM-grass associations than that which occurs in tree-grass associations ( $\mathrm{Yu}$ and D'Odorico, 2014b). Changes in the partitioning of total evapotranspiration to different components (i.e., evaporation and transpiration) induced by a low transpiration rate of CAM plants have been confirmed in some experimental evidence (Zou et al., 2010; Bacilio et al., 2011). Consistent with these results, field experiments have found that a deeprooted CAM species, Yucca schidigera, performs hydraulic lift within a $350 \mathrm{~mm}$ deep root zone in (Yoder and Nowak, 1999).

This study also evaluates the effects of root hydraulic conductivity, mesophyll conductance, and rainfall regime on hydraulic redistribution by CAM plants at the growing season to yearly timescale (Figure 7.2a, b; Supplementary Figure 7.1a, b). As expected, I find that climate (i.e. rainfall regime) exerts a relatively strong control over HR at the growing season to yearly timescale (Yu and D'Odorico, 2014b). My study focuses on the case in which deep soil moisture is supplied by surface soil water drainage into the deep soil layer. An increase in root hydraulic conductivity allows for a quicker transport of water instantaneously (a short time scale) but will leave less water available for subsequent water redistribution in response to a water potential gradient. This is in contrast to other studies in which hydraulic lift relies on deep soil moisture supplied by groundwater (Mooney et al., 1980; Ryel et al., 2002; Hultine et al., 2004). For instance, Ryel et al (2002) evaluated the effects of groundwater on hydraulic lift. In their study, the water potential in the deeper soil layers was taken to be constant, which can lead to an 
increase of hydraulic lift with an increase in root hydraulic conductivity. The relatively low mesophyll conductance is a typical characteristic for CAM plants (e.g., Flexas et al., 2008; Nelson and Sage, 2008; Ripley et al., 2013). This study shows that reduction in mesophyll conductance by CAM plants reduces their transpiration rate (eq. (5)) and increases transpiration in grasses growing in the shallow soil, thereby sustaining water potential gradient favorable for hydraulic lift.

The modeling results also show that trees in tree-CAM associations perform a higher rate of hydraulic descent than those in tree-grass associations in dry environment (Figure 7.2a), resulting from a lower transpiration rate by CAM plants in the shallow soil layer. Trees have been widely documented to facilitate the seedling establishment of CAM plants in drylands through an increase in soil resource availability (i.e. water and nitrogen) (e.g., Withgott, 2000; Castillo and Valiente-Banuet, 2010; Cares et al., 2013). Hydraulic descent transports water deeper into the soil column, thereby leading to less soil water available for shallow-rooted CAM plants (Burgess et al., 2001; Hultine et al., 2004) and disfavoring the seeding establishment and/or growth of CAM plants.

In fact, the modelling results show the ability of relatively high hydraulic descent rates by woody plants to turn CAM plant facilitation into competition (Yu and D'Odorico, in press). These results may improve my understating of traditional niche differentiation theory (e.g., Walter, 1971; Walker and Noy-Meir, 1982). In fact, a stronger allocation of woody plant roots into deeper soil layers (i.e., increase in niche differentiation with shallow rooted plants) could still exert a competitive effect on shallow rooted plants through the mechanism of hydraulic descent. Hydraulic descent could be an adaptive strategy trees take to invest in deep roots when soils have a relatively low hydraulic conductivity, thereby limiting the drainage rates (e.g., 
Burgess et al., 2001; Ryel et al., 2004). The rate of hydraulic descent increases as the root depth of woody plants increases; in these conditions trees exert a competitive rather than a facilitative effect on CAM plants in access to soil water. In fact, it has been proposed that CAM plants that are facilitated during their establishment stage competes with and even excludes their nurse plants (woody plants) as an adult (e.g., Withgott, 2000) due to ontogenetic changes in the interacting species, but the experimental evidences are scare (e.g., Flores-Martinez et al., 1994; Flores-Torres and Montana, 2015). The role of hydraulic descent in turning facilitation of CAM plants by woody plant to competition as shown in this study may explain why CAM plants (which benefit from the presence of trees during their establishment), once they become adults they are not strong enough to outcompete their nurse plants (i.e., trees), consistent with field observations (i.e., Reyes-Olivas et al., 2002; Flores-Torres and Montana, 2015). In view of the universal ability of woody plants to perform hydraulic redistribution (e.g., Neumann and Cardon, 2012; Prieto et al., 2012), the role of hydraulic descent benefiting trees may contribute to my understanding tree-CAM association/coexistence widely documented in different arid and semiarid zones including the Sonoran and Chihuahuan deserts (e.g., Hutto et al., 1986; Drezner, 2007; Flores-Torres and Montana, 2015), the Zapotitlán de las Salinas or Tehuacan valley, central Mexico (e.g., Valiente-Banuet et al., 1991), coastal deserts in northern Sinaloa, Mexico (e.g., Reyes-Olivas et al., 2002), and Chilean arid and semiarid zones (e.g., Cares et al., 2013).

Hydraulic redistribution clearly has the ability to affect interspecies interactions and plant community composition. This study evaluates the effects of hydraulic redistribution on transpiration at the patch scale for tree-grass, CAM-grass, and tree-CAM associations. This has been largely ignored by past studies, which focused on the effect of HR on tree (or shrub)-grass and tree-shrub interactions (Ludwig, 2004; Zou et al., 2005; Quijano et al., 2012; Yu and 
D’Odorico, 2014b). I found an increase in transpiration with HR (hydraulic descent, Figure 7.3a) in dry environments and a reduction in transpiration with HR (hydraulic lift, Figure 7.3c) in relatively wet environments for deep-rooted plants. The results were opposite for shallow-rooted plants (Figure 7.3b-d). These results are consistent with Yu and D'Odorico (2014b) who found that hydraulic lift reduces the water stress of shallow-rooted plants (i.e. grasses) while increasing the water stress of deep-rooted plants (i.e. trees). The benefits gained by shallow-rooted plants in scavenging lifted water have been documented in experimental studies (Richards and Caldwell, 1987; Zou et al., 2005; Brooks et al., 2006). More recent studies indicate that shallow-rooted plants can directly take up lifted water through ecto- and endomycorrhizal networks (CMNs) (EgertonWarburton et al., 2007; Warren et al., 2008). However, the hydraulic lifter itself may be a strong competitor for lifted water and thus competition may dominate (Ludwig et al., 2004). In contrast, hydraulic descent increases the transpiration rate of deep-rooted plants through transporting water deeper into the soil column, reducing losses by soil evaporation, and precluding competition for water with shallow-rooted plants (Burgess et al., 2001; Hultine et al., 2004; Ryel et al., 2004).

In summary, this study highlights the implications of hydraulic redistribution performed by CAM plants on plant community dynamics. The modeling results show a higher rate of hydraulic lift (HL) by CAM plants in CAM-grass associations as compared to HL by trees in tree-grass associations in relatively wet environments. The increased HL in CAM-grass associations may benefit grasses, which could utilize the lifted water. The modeling results also show a higher rate of hydraulic descent by trees in tree-CAM associations as compared to tree-grass associations in dry environments. The increased hydraulic descent may benefit the trees in tree-CAM associations. By moving water deep into the soil column, trees can reduce their water competition with surrounding plants. These small-scale interactions may scale up to affect vegetation dynamics (i.e., 
biomass and vegetation cover) and the resiliency of plant communities, especially when considering the interactions between the land surface (i.e., vegetation cover) and the atmosphere (e.g., rainfall). 


\title{
8 Direct and indirect facilitation of plants with Crassulacean Acid Metabolism (CAM)
}

This chapter is adapted from Yu KL and D'Odorico P. (2105), Direct and indirect facilitation of plants with Crassulacean Acid Metabolism (CAM), Ecosystems, 2015, 18:985-999.

\begin{abstract}
Plants with Crassulacean Acid Metabolism (CAM) are increasing their cover in many dryland regions around the world. Their increased dominance has been related to climate warming and atmospheric $\mathrm{CO}_{2}$ fertilization, while the effect of interspecies interactions and the role of CAM plant facilitation by trees and grasses remain poorly understood. Woody plants are known for their ability to directly facilitate CAM plants through amelioration of the abiotic environment. Mechanisms of indirect facilitation of trees on CAM plants in tree-grass-CAM associations, however, have received less attention. It is also unclear whether grasses might facilitate CAM plants in mixed tree-grass-CAM communities. For instance, the inclusion of grasses in tree-CAM associations could enhance hydraulic lift and facilitate CAM plants in their access to shallow soil moisture at the expenses of deep rooted trees. If this effect outweighs the competitive effects of grasses on CAM plants, grasses could overall facilitate CAM plants through hydraulic lift. Here I develop a process-based ecohydrological model to investigate the direct and indirect facilitation in tree-CAM-grass associations; the model quantifies transpiration of CAM plants when isolated as well as in associations with trees and/or grasses. It is found that woody plants having a high root overlap with CAM plants indirectly facilitate CAM plants by significantly reducing grass transpiration in shaded conditions. For situations of a low to moderate root overlap, facilitation may occur both directly and indirectly. Conversely, grasses are unable to indirectly facilitate CAM plants through the mechanism of hydraulic lift because the competitive effects of grasses on CAM plants outweigh the facilitation induced by hydraulic lift.
\end{abstract}




\subsection{Introduction}

Plants with Crassulacean Acid Metabolism (CAM) are increasing their abundance in many drylands around the world (Borland et al., 2009, 2011). This effect is typically related to changes in climate or increasing atmospheric $\mathrm{CO}_{2}$ concentrations (e.g., Drennan and Nobel, 2000; Borland et al., 2009), while the role of interactions with other species and the relationship with other ongoing changes in plant community composition (e.g., woody plant encroachment and grass invasions) remain not completely understood. The role of facilitation or positive interactions has been increasingly emphasized in plant community studies in the past few decades (Bruno et al., 2003; Brooker et al., 2008), especially under high levels of environmental stress (Callaway et al., 2002; Maestre et al., 2009). A common example of facilitation that has been widely documented is the nurse effect of woody plants on CAM plants in drylands (Withgott, 2000; Castillo and Valiente-Banuet, 2010; Cares et al., 2013). Studies of CAM plants engender scientific interest because their photosynthetic plasticity can buffer fluctuations in environmental conditions (Borland et al., 2009, 2011). Renewed interest in CAM plants is further contributed by their suitability as feedstock for bioenergy production in drylands (Borland et al., 2009; Davis et al., 2011).

Past studies have largely focused on the direct facilitation of woody plants on CAM plants, whereby woody plants increase the establishment rate of CAM plants by increasing soil resource availability (water and/or nitrogen) and/or providing refuge from physical stress under extreme environmental conditions (temperature and/or solar radiation) (Withgott, 2000; Castillo and Valiente-Banuet, 2010; Cares et al., 2013). Indirect facilitation of woody plants on CAM plants, however, remains poorly investigated. Indirect facilitation involves three interacting species in which competitive species A suppresses species B and thus reduces the competitive effect of 
species B over species C (Levin, 1999; Kunstler et al., 2006; Brooker et al., 2008). Studies suggest that indirect facilitation tends to occur in a system where pairs of plants $(\mathrm{A}-\mathrm{B}, \mathrm{B}-\mathrm{C})$ compete for different resources (Levin, 1999; Pagès et al., 2003; Callaway, 2007; Brooker et al., 2008). For example, woody plants suppress the growth of herbaceous vegetation through light competition (A-B) and thus lead to competitive release of soil nutrients (water and/or nitrogen) which favors a third species (B-C) (Levin, 1999; Siemann and Rogers, 2003; Kunstler et al., 2006).

Experimental evidence of indirect facilitation among species of different trophic levels has been extensive (e.g., Rousset and Lepart, 2000; Corcket et al., 2003; Boulant et al., 2008; Anthelme and Michalet, 2009); fewer studies have investigated indirect facilitation among species within the same trophic level, especially in arid and semiarid systems (Brooker et al., 2008; Cuesta et al., 2010). This is presumably due to the simultaneous occurrence of direct facilitation (Miller, 1994; Siemann and Rogers, 2003) and the difficulty in interpreting the results of experiments in which more than one species is manipulated (Callaway, 2007). In fact, species A can also compete with species $\mathrm{C}$ and thus indirect facilitation requires that the indirect facilitative effect through suppression of a shared competitor outweighs the direct competitive effect (Levin 1999; Brooker et al., 2008). For instance, Pagès et al (2003) and Pagès and Michalet (2003) found that the direct negative effect of species A on species C through light reduction outweighs the indirect positive effect of competitive release. Some models have explored the indirect facilitation among species within the same trophic level (e.g., Lawlor, 1979; Vandermeer, 1990; Stone and Roberts, 1991), but their approach has been mostly theoretical with no reference to specific functional groups. Here I develop a process-based model 
to investigate the emergence of indirect facilitation within dryland plant communities with three functional groups: $\mathrm{C}_{3}$ woody plants, $\mathrm{C}_{4}$ grasses, and CAM plants.

$\mathrm{C}_{4}$ grasses sustain a high additional metabolic cost for photosynthesis in hot and/or dry environments, and thus tend to be shade intolerant (e.g., Siemann and Rogers, 2003; Sage and McKown, 2006; Borland et al., 2009). In contrast, the photosynthetic plasticity of CAM plants and their acclimation to shade allows them to be shade tolerant (Medina et al., 1986; Fetene et al., 1990; Ceusters et al., 2011). Grasses and CAM plants are typically shallow rooted (Ogburn and Edwards, 2010; Nippert et al., 2012) and thus they compete for soil water resources. Thus, in tree-CAM-grass associations, it may be straightforward to expect that trees can suppress grasses through light reduction (e.g., Siemann and Rogers, 2003; Kunstler et al., 2006) and thus indirectly facilitate CAM plants (Figure 1a, 1b). This may imply that the widely documented phenomenon of woody plant encroachment (Van Auken, 2000; D’Odorico et al., 2012) can directly and/or indirectly exert a positive net effect on CAM plant productivity in drylands.

What remains unclear is how tree-CAM associations responds to increase in grass density (grass invasions). Grasses will compete with CAM plants, but increase in grass transpiration and root uptake from the shallow soil layer are expected to promote the occurrence of hydraulic lift (e.g., Yu and D'Odorico, 2014b). Hydraulic lift transports water from the wetter deep soil to the drier shallow soil through plant roots (Richards and Caldwell, 1987; Ludwig et al., 2003). Shallow rooted plants have been found to be capable of scavenging the lifted water (Richards and Caldwell, 1987; Zou et al., 2005; Brooks et al., 2006). Thus, it has been suggested that hydraulic lift contributes to facilitation of deep rooted plants on shallow rooted plants (Riginos et al., 2009; Moustakas et al., 2013; Dohn et al., 2013) at the expense of deep rooted plants (Yu and D'Odorico, 2014b). But, can the benefit to CAM plants associated with hydraulic lift induced by 
grass invasions outweigh the competitive effect? In other words, can the introduction of grasses into tree-CAM associations indirectly facilitate CAM plants through the mechanism of hydraulic lift (Figure 1c)?

(a)

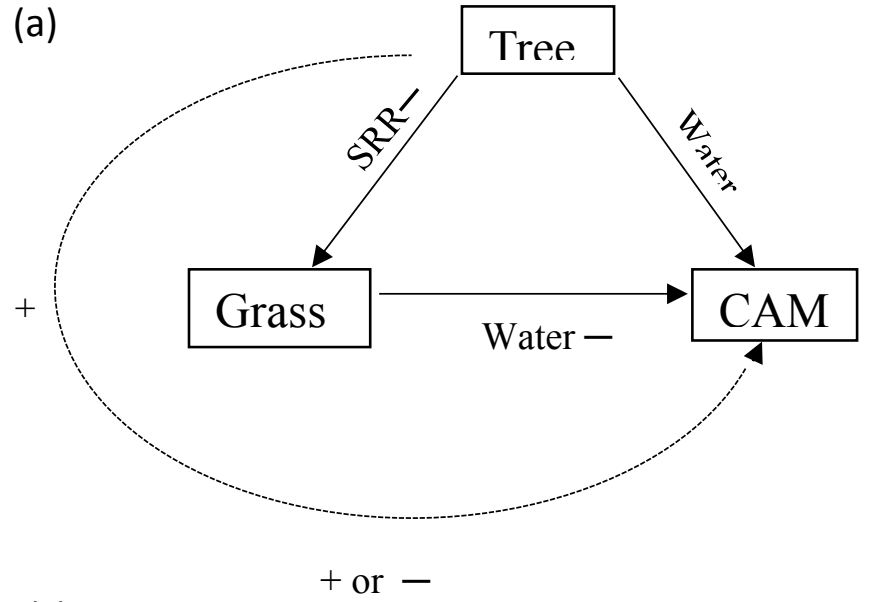

(c)

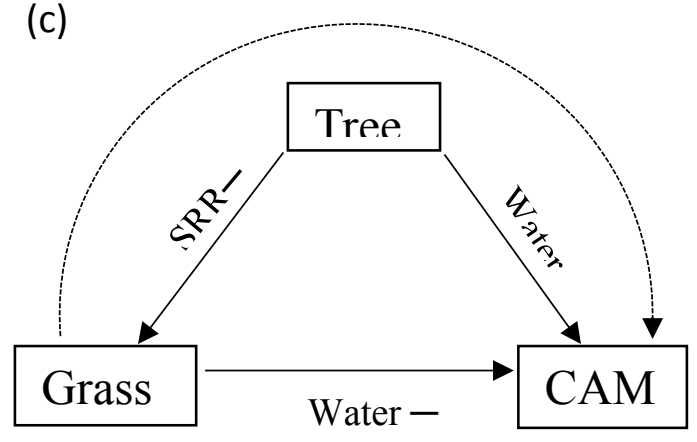

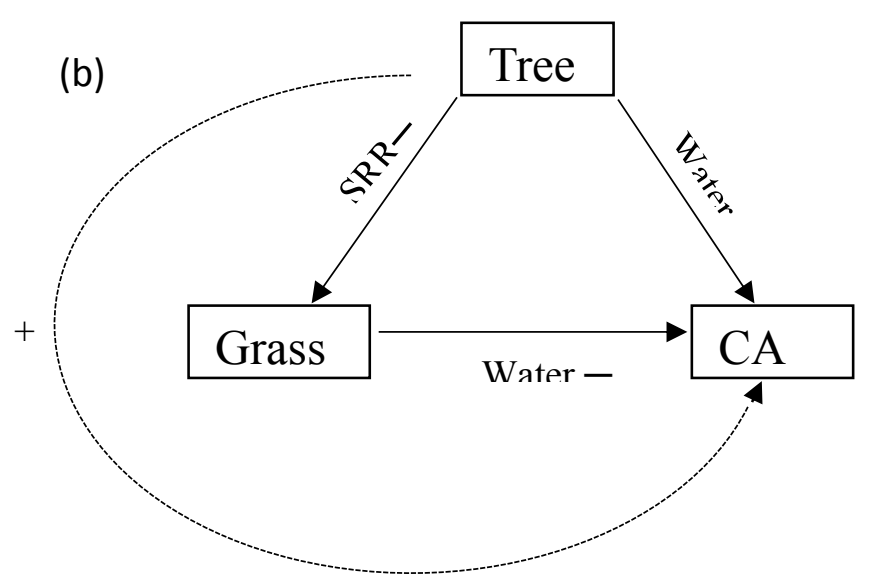

Figure 8-1: Schematic diagram of indirect (dotted line) and direct (solid line) interactions among C3 trees, C4 grasses, and CAM plants. $(\mathrm{a}, \mathrm{b})$ trees suppress grass transpiration through solar radiation reduction (SRR) and reduce the competitive effect of grasses on CAM plants in access to soil water resources, thus indirectly facilitating CAM plants. (a) Trees and CAM plants compete for soil water resources because of a high degree of root overlap; (b) trees directly facilitate CAM plants in situations of a low to moderate root overlap. (c) Inclusion of grasses in tree-CAM associations increases hydraulic lift suppressing trees and thus may indirectly facilitate CAM plants.

In this study, I develop a model to investigate the occurrence of direct and indirect facilitation in tree-CAM-grass associations along a rainfall gradient. I quantify CAM plant transpiration here used as an indicator of water availability - in CAM plants alone, CAM-grass, tree-CAM, 
and tree-CAM-grass associations, at seasonal-to-annual timescales. By clarifying the role of direct and indirect facilitation in the tree-CAM-grass associations, my study contributes to the understanding of dynamics of CAM plants in response to important global environmental change phenomena, such as woody plant encroachment and/or grass invasions.

\subsection{Methods}

\subsubsection{Modelling framework}

I develop a model to investigate the direct and indirect facilitation in tree-CAM-grass associations along a rainfall gradient. The model simulates soil moisture dynamics in two soil layers and accounts for flows between them due to drainage and hydraulic redistribution $(H R)$ (Ryel et al., 2002; Lee et al., 2005; Yu and D'Odorico, 2014b). It quantifies transpiration of CAM plants in CAM plants alone (C), CAM-grass (C-G), tree-CAM (T-C), and tree-CAM-grass (T-C-G) associations, at seasonal-to-annual timescales (Table 1).

Table 8-1 A summary of vegetation associations in this study.

\begin{tabular}{llll}
\hline $\begin{array}{l}\text { Vegetation } \\
\text { associations }\end{array}$ & $\begin{array}{l}\text { Deep rooted } \\
\text { plants }\end{array}$ & $\begin{array}{l}\text { Shallow rooted } \\
\text { plants }\end{array}$ & $\begin{array}{l}\text { Plants performing } H R \text { at } \\
\text { night }\end{array}$ \\
\hline CAM & & CAM & \\
CAM-Grass & CAM; Grass & Tree \\
Tree-CAM & Tree & CAM & Tree \\
Tree-CAM-Grass & Tree & CAM; Grass & \\
\hline
\end{tabular}

Transpiration can be linked to total $\mathrm{CO}_{2}$ assimilation and hence to plant fitness. A lower transpiration of CAM plants in CAM-grass (C-G) and tree-CAM (T-C) associations than in CAM plants alone $(\mathrm{C})$ indicates the competitive effects of grasses and trees on CAM plants and 
vice versa. A comparison of transpiration of CAM plants in tree-CAM-grass (T-C-G) and CAMgrass (C-G) associations can explain whether trees directly or indirectly facilitate CAM plants. A comparison of CAM plant transpiration in tree-CAM-grass (T-C-G) and tree-CAM (T-C) associations will indicate whether grasses indirectly facilitate CAM plants through the mechanism of hydraulic lift. To this end, in the following subsections I define the CAM transpiration ratios (see below for details). I focus on the case in which roots of CAM plants and grasses grow only in the shallow soil layer (Ogburn and Edwards, 2010; Nippert et al., 2012) while roots of woody plants (trees) are present in both the shallow and deep soil layers (Yu and D’Odorico, 2014b). Woody plants and grasses transpire in the daytime (12 hours) and woody plants perform $H R$ at night (12 hours) (Ryel et al., 2002; Lee et al., 2005; Yu and D’Odorico, 2014b), while CAM plants are assumed to transpire only at night (12 hours) (Lüttge, 2004; Ogburn and Edwards, 2010). Some facultative CAM plants can actually perform regular $\mathrm{C}_{3}$ photosynthesis and thus also transpire during daytime (e.g., Borland et al., 2011). This effect can be easily accounted for by varing the duration transpiration in facultative CAM plants. In this study, however, I will focus on the case of obligated CAM plants. To account for a nonnegligible plant water capacitance of CAM plants (Lüttge, 2004; Ogburn and Edwards, 2010), I account for changes in water storage in CAM plants (e.g., Lhomme et al., 2001; Bartlett et al., 2014).

\subsubsection{Water balance}

Soil moisture dynamics in the two soil layers for tree-CAM (T-C) and tree-CAM-grass (T-C-G) associations are modeled by two coupled equations

$$
n Z_{1} \frac{d s_{1}}{d t}=P-U_{1}-E-D_{1}+H R
$$


and

$$
n Z_{2} \frac{d s_{2}}{d t}=D_{1}-U_{2}-D_{2}-H R
$$

where the subscripts 1 and 2 refer to the shallow and deep soil layer, respectively; $n$ is the soil porosity, $Z_{1}$ and $Z_{2}$ the soil layer thickness $(\mathrm{mm}), S_{1}$ and $S_{2}$ the relative soil moisture $\left(0<S_{1}\right.$, $\left.S_{2} \leq 1\right), P$ the rate of rainfall infiltration into the top soil layer $\left(\mathrm{mm} \mathrm{d}^{-1}\right), U_{1}$ and $U_{2}$ the soil moisture losses from each soil layer due to root uptake $\left(\mathrm{mm} \mathrm{d}^{-1}\right), E$ the evaporation rate from the soil surface $\left(\mathrm{mm} \mathrm{d}^{-1}\right), D_{1}$ and $D_{2}$ the drainage rates $\left(\mathrm{mm} \mathrm{d}^{-1}\right)$, and $H R$ the hydraulic redistribution at the patch scale $\left(\mathrm{mm} \mathrm{d}^{-1}\right)$. Positive values of $H R$ indicate "hydraulic lift" (i.e., upward hydraulic redistribution), while negative values of $H R$ indicate "hydraulic descent" (i.e., downward hydraulic redistribution). For CAM plants alone (C) and CAM-grass associations (CG), only equation 1 needs to be used to quantify soil moisture dynamics, where $H R$ is taken to be $0 \mathrm{~mm} \mathrm{~d}^{-1}$ because in these two cases there are no deep rooted plants to perform $H R$.

Precipitation is modeled as a sequence of intermittent rainfall events occurring as a marked Poisson process with average rainfall frequency, $\lambda$, (events per day). The depth (mm) of each storm is modeled as an exponentially distributed random variable with mean, $h$, (mm per event) (Rodriguez-Iturbe et al., 1999a). Runoff occurs when the surface layer is saturated (i.e., $S_{1}=1$ ). Drainage is assumed to be driven only by gravity and is expressed as $D=\frac{K_{s}\left(\exp \left(\beta\left(S-S_{f c}\right)-1\right)\right.}{\exp \left(\beta\left(1-S_{f c}\right)-1\right)}$, where $K_{S}$ is the soil saturated hydraulic conductivity $\left(\mathrm{mm} \mathrm{h}^{-1}\right), \beta$ a coefficient, $S$ the relative soil moisture, and $S_{f c}$ the field capacity (Laio et al., 2001).

Uptake by woody plants and grasses is determined assuming that steady-state exists within the soil-plant-atmosphere continuum and therefore uptake is taken equal to transpiration 
(Porporato et al., 2003; Manzoni et al., 2013). The maximum total potential evapotranspiration in the daytime is assumed to be constant (Supplementary Table 1). Transpiration of CAM plants does not occur during daytime (Lüttge, 2004; Ogburn and Edwards, 2010). Therefore, for CAM plants alone $(\mathrm{C})$, the maximum total potential evapotranspiration in the daytime $\left(E T_{\text {maxd }}\right)$ is contributed only by the potential evaporation at the soil surface $\left(E_{\text {maxd }}\right)$. For the CAM-grass associations $(\mathrm{C}-\mathrm{G}), E T_{\operatorname{maxd}}$ is partitioned into potential transpiration for grasses $\left(T_{\text {gmaxd }}\right)$ and potential evaporation at the soil surface $\left(E_{\text {maxd }}\right)$, where $T_{\text {gmaxd }}$ depends on grass cover $\left(f_{g}\right)$, as

$$
T_{\text {gmaxd }}=E T_{\text {maxd }} f_{g}
$$

For the tree-CAM associations, $E T_{\operatorname{maxd}}$ is partitioned to potential transpiration for trees $\left(T_{\text {tmaxd }}\right)$ and potential evaporation from the soil surface $\left(E_{\operatorname{maxd}}\right)$. For the tree-CAM-grass associations, $E T_{\text {maxd }}$ is partitioned into potential transpiration for trees $\left(T_{\text {tmaxd }}\right)$ and grasses $\left(T_{\text {gmaxd }}\right)$, and potential evaporation from the soil surface $\left(E_{\operatorname{maxd}}\right)$. To account for the solar radiation reduction by trees, the incident shortwave radiation is assumed to vertically irradiate the plant and soil surfaces (Caylor et al., 2005; Yu and D’Odorico, 2014 a b). Potential evapotranspiration depends on the available shortwave radiation, which exponentially decays through the tree canopy according to Beer's law. Therefore, following Caylor et al (2005) and Yu and D'Odorico (2014 a b), for the tree-CAM associations, I have $T_{\text {tmaxd }}=E T_{\operatorname{maxd}}\left[1-\exp \left(-k_{s} L A I_{t}\right)\right]$ and $E_{\text {maxd }}=$ $E T_{\text {maxd }} \exp \left(-k_{s} L A I_{t}\right)$, where $k_{s}$ is the extinction coefficient of shortwave radiation, and $L A I_{t}$ the leaf area index of woody plants $\left(\mathrm{m}^{2} \mathrm{~m}^{-2}\right)$. Likewise, for the tree-CAM-grass associations (TC-G), I have

$$
\begin{aligned}
& T_{\text {tmaxd }}=E T_{\text {maxd }}\left[1-\exp \left(-k_{s} L A I_{t}\right)\right] \\
& T_{\text {gmaxd }}=E T_{\text {maxd }} \exp \left(-k_{s} L A I_{t}\right) f_{g}
\end{aligned}
$$




$$
E_{\text {maxd }}=E T_{\text {maxd }} \exp \left(-k_{s} L A I_{t}\right)\left(1-f_{g}\right)
$$

A comparison between equation (3) and (5) shows that trees reduce shortwave radiation and thus decrease the grass transpiration rate even when the grass cover remains the same as in the case with no trees.

Potential transpiration for trees $\left(T_{\operatorname{tmaxd}}\right)$ is contributed by the shallow soil layer $\left(T_{1 t d \max }\right)$ and the deep soil layer $\left(T_{2 t d \max }\right)$; this two fractions are assumed to be proportional to the water volume available in each layer:

$$
\begin{gathered}
T_{1 t \text { dmax }}=T_{\text {tdmax }} \frac{Z_{1} S_{1}}{Z_{1} S_{1}+Z_{2} S_{2}} \\
T_{2 t \text { max }}=T_{\text {tdmax }} \frac{Z_{2} S_{2}}{Z_{1} S_{1}+Z_{2} S_{2}}
\end{gathered}
$$

(Yu and D'Odorico, 2014 a). The actual transpiration by plants depends on the soil water availability (Rodriguez-Iturbe et al., 1999a); I express the limitation of transpiration by soil water availability as

$$
\tau(\mathrm{S})=\left\{\begin{array}{cc}
0, & S<S_{w} \\
\frac{S-S_{w}}{S^{*}-S_{w}}, & \text { and } S<S^{*} \\
1, & \text { and } S \geq S^{*}
\end{array}\right.
$$

where $\tau(\mathrm{S})$ expresses soil moisture limitations on evapotranspiration, $S$ is the soil moisture, $S^{*}$ is the vegetation-specific value of relative soil moisture above which transpiration is not limited by soil water availability, and $S_{w}$ is the vegetation-specific wilting point at which transpiration ceases. Trees and grasses are assumed to have the same $S^{*}$ and $S_{w}$. Therefore, the actual 
transpiration of woody plants in the shallow $\left(T_{1 t d a}\right)$ and deep $\left(T_{2 t d a}\right)$ soil layers are determined as

$$
\begin{gathered}
T_{1 t d a}=T_{1 t d \max } \tau\left(S_{1}\right) r_{1},(9) \\
T_{2 t d a}=T_{2 t d \max } \tau\left(S_{2}\right) r_{2},(10)
\end{gathered}
$$

where $r_{1}$ and $r_{2}$ are the cumulated (and normalized) tree root densities in the shallow and the deep soil layers, respectively $\left(r_{1}+r_{2}=1\right)$. The actual transpiration by grasses $\left(T_{1 g d a}\right)$ is determined as $T_{1 g d a}=T_{\text {gmaxd }} \times \tau\left(S_{1}\right)$. As seen from equations (7), (8), (9), and (10), a high degree of overlap between the roots of trees and CAM plants are characterized by high values of $Z_{1} / Z_{2}$ and $r_{1} / r_{2}$ and is expected to lead to the competitive effects of trees on CAM plants.

Uptake by CAM plants is determined using a non-steady-state approach. Following other studies (e.g., Lhomme et al., 2001; Bartlett et al., 2014), I model the non-steady-state plant water storage by incorporating capacitances and resistances into the water flow pathway similarly to the case of electric circuits (see Figure 1 in Chapter 7). In this method, the rates of water uptake $\left(U_{C A M}\right)$ and the plant water capacitance $\left(Q_{w}\right)$ balance the leaf transpiration $\left(T_{C A M}\right)$ per unit ground area. Therefore, I have

$$
T_{C A M}=U_{C A M}+Q_{w},
$$

Following Bartlett et al., (2014), $U_{C A M}$ and $Q_{w}$ are controlled by water potential gradients, with $U_{C A M}=g_{s r p}\left(\Psi_{s 1}-\Psi_{x}\right)$ and $Q_{w}=g_{c} L A I_{c}\left(\Psi_{w}-\Psi_{x}\right)$, where $g_{s r p}$ is the soil-root-plant conductance per unit ground area $\left(\mathrm{m} \mathrm{s}^{-1} \mathrm{MPa}^{-1}\right), g_{c} L A I_{c}$ the storage conductance per unit ground area $\left(\mathrm{m} \mathrm{s}^{-1} \mathrm{MPa}^{-1}\right)\left(g_{c}\right.$ is storage conductance per unit leaf area and $L A I_{c}$ is leaf area index of CAM plants), $\Psi_{s 1}$ the soil water potential in the shallow soil layer, $\Psi_{x}$ the xylem water potential 
(MPa), $\Psi_{w}$ the plant storage water potential (MPa). $T_{C A M}$ is the flux from the xylem to the leaves, which can be expressed as

$$
T_{C A M}=\frac{g_{p} L A I_{c}}{1-f}\left(\Psi_{x}-\Psi_{l}\right)(12),
$$

where $g_{p}$ is the plant conductance per unit leaf area, $f$ the fraction of plant resistance below the storage branch connection, $\frac{g_{p} L A I_{c}}{1-f}$ the plant conductance per unit ground area between the storage connection node (with water potential, $\Psi_{x}, \mathrm{MPa}$ ) and leaf (with water potential, $\Psi_{l}$, $\mathrm{MPa})$.

The leaf transpiration $\left(T_{C A M}\right)$ per unit ground area can be also calculated (e.g., Bartlett et al., 2014) as a function of the specific humidity gradient between the leaf mesophyll $\left(q_{l}\right)$ and the atmosphere $\left(q_{a}\right)$, i.e.,

$$
T_{C A M}=\lg _{m s a} \frac{\rho_{a}}{\rho_{w}}\left(q_{l}-q_{a}\right)
$$

where $\rho_{a}$ is the density of air $\left(\mathrm{Kg} \mathrm{m}^{-3}\right), \rho_{w}$ the density of water $\left(1 \mathrm{Kg} \mathrm{m}^{-3}\right), g_{m s a}$ the series of the mesophyll, stomatal, and atmospheric conductances $\left(\mathrm{m} \mathrm{s}^{-1}\right)$ to water vapor per unit ground under well watered conditions (i.e., $g_{m} L A I_{c}, g_{s} L A I_{c}$, and $g_{a}$, respectively); thus $g_{m s a}$ can be expressed as $g_{m s a}=L A I_{c} g_{m} g_{s} \frac{g_{a}}{L A I_{c} g_{m} g_{s}+g_{s} g_{a}+g_{m} g_{a}}$. In equation (13), $l$ is a coefficient limiting $g_{m s a}$ in dry conditions, while $q_{l}$ is a function of $\Psi_{l}$ and leaf temperature. Detailed calculations of parameters $g_{s r p}, \Psi_{w}, g_{m s a}, l, q_{l}$ and other parameters can be found in Bartlett et al., (2014). The rate of CAM plant uptake is then calculated combing equations (11)-(13) as in Bartlett et al., (2014) with equation (13) driven by atmospheric conditions. 
Actual evaporation from soil surface $(E)$ also depend on soil water availability. Consistent with Porporato et al (2003) and Bartlett et al (2014), I have

$$
E=\left\{\begin{array}{c}
0, \quad 0 \leq S \leq S_{h} \\
E_{\max } \frac{S-S_{h}}{1-S_{h}},{\text { and } S_{h}}<S<1
\end{array}\right.
$$

where $S_{h}$ is the hygroscopic point below which evaporation at the soil surface ceases (Laio et al., 2001), $E_{\max }$ the potential evaporation during the daytime or at night. The daytime potential evaporation is calculated with eq. (6), while the total potential evaporation from the soil surface at night $\left(E_{\operatorname{maxn}}\right)$ is assumed to be constant (Supplementary Table 1).

Consistent with other studies (Ryel et al., 2002; Lee et al., 2005; Yu and D’Odorico, 2014b), hydraulic redistribution is determined as $\mathrm{HR}=c C_{r \max }\left(\Psi_{s 2}-\Psi_{s 1}\right) \min \left(r_{1}, r_{2}\right)$, where $C_{r \max }$ is the maximum root hydraulic conductance of the entire active root system $\left(\mathrm{mm} \mathrm{MPa}^{-1} \mathrm{~h}^{-1}\right), c$ a factor reducing root hydraulic conductance and a function of soil water potential (Supplementary Table 1), $\Psi_{s 2}$ and $\Psi_{s 1}$ the soil water potential (MPa) in the deep and the shallow soil layers, respectively. $\Psi$ is determined as $\Psi=\Psi_{S} \times S^{-d}$, where $\Psi$ is the soil water potential, $S$ soil moisture, while $\Psi_{S}$ and $d$ are experimentally derived parameters that have been determined for a variety of soils (Supplementary Table 2) (Clapp and Hornberger, 1978). The detailed calculations of $c$ can be found in Yu and D'Odorico (2014b).

\subsubsection{CAM plant transpiration ratios}

To compare the different levels of water stress in CAM plants in different associations with other

functional types, I define the transpiration ratios as $\xi=\frac{T_{1 C}(\mathrm{Cas})}{T_{1 C}(\mathrm{C})}$, where $T_{1 C}(\mathrm{Cas})$ and $T_{1 C}(\mathrm{C})$ are transpiration of CAM plants in CAM associations (with trees, grasses, or both) and CAM plants 
alone, respectively. Likewise, to evaluate whether grasses indirectly facilitate CAM plants, I define the transpiration ratio $(\xi)$ between tree-CAM-grass associations (T-C-G) and tree-CAM associations (T-C) as $\xi=\frac{T_{1 C}(\mathrm{TCG})}{T_{1 C}(\mathrm{TC})}$, where $T_{1 C}(\mathrm{TCG})$ and $T_{1 C}(\mathrm{TC})$ are the transpiration of CAM plants in tree-CAM-grass association and tree-CAM associations, respectively.

\subsubsection{Parameterization of the model}

The model is mainly parameterized with respect to environmental conditions with two rainfall regimes corresponding to arid $\left(\lambda=0.2 \mathrm{~d}^{-1}\right.$ and $\left.h=5 \mathrm{~mm}\right)$ and semiarid $\left(\lambda=0.2 \mathrm{~d}^{-1}\right.$ and $h=10$ $\mathrm{mm}$ ) environments. Soil moisture dynamics are simulated with a time step of half an hour for ten years. The transpiration of CAM plants in CAM associations and CAM alone is averaged over ten years and then used to calculate the transpiration ratios defined above. Other variables such as evapotranspiration and hydraulic redistribution are also reported as average values over ten years. The growing seasons of trees, grasses and CAM plants are assumed to coincide and last 210 days each year (Bhattachan et al., 2012). The root depth of CAM plants and grasses is assumed to be the same and constant $\left(Z_{1}=10 \mathrm{~cm}\right)$ in all the simulations (Ogburn and Edwards, 2010; Nippert et al., 2012). To investigate whether a high degree of root overlap leads to the competitive effects of trees on CAM plants (Figure 1a), low values of deep soil layer thickness $\left(Z_{2}=10 \mathrm{~cm}\right)$ and root allocation to the deep soil $\left(r_{2} / r_{1}=0.2\right)$ are used, thus precluding the occurrence of hydraulic distribution (Caldwell et al., 1998; Espeleta et al., 2004). Conversely (Figure $1 \mathrm{~b}, \mathrm{c}$ ), woody plants with deeper roots (i.e., $Z_{2}=30 \mathrm{~cm}$ ) can perform hydraulic redistribution; these conditions allow us to evaluate the role played by hydraulic redistribution in the direct and/or indirect facilitation in tree-CAM-grass associations. This model is mainly implemented in loamy sand and results of sensitivity analysis of sandy loam are provided in 
Supplementary Material. Parameters describing various soil characteristics used in this study can be found in Supplementary Table 2. The maximum root hydraulic conductance of woody plants for the entire active root system $\left(C_{r \max }\right)$ is taken to be $C_{r \max }=0.75 \mathrm{LAI}_{t} \mathrm{~mm} \mathrm{MPa}^{-1} \mathrm{~h}^{-1}$, following Lee et al (2005) and Yu and D'Odorico (2014b). Other parameters required in this study can be found in Supplementary Table 1 . This study does not explicitly account for the effects of canopy interception in the soil moisture balance. Canopy interception in CAM associations could be higher than that in CAM plants alone. To account for the effect of canopy interception, I rerun the model in which rainfall in excess of canopy interception is available for infiltration in the soil moisture balance. By this, I calculated the canopy interception (CI) as $\mathrm{CI}=0.2 \times \mathrm{LAI}$ (Yu et al., 2012), where LAI is leaf area index of canopies. Grass cover is taken to be $70 \%$ both in arid and semiarid environments in this study and LAI of grasses is taken to be $2.5 \mathrm{~m}^{2} \mathrm{~m}^{-2}$. LAI of trees and CAM plants can be found in Supplementary Table 1. Some grasses have deeper roots (i.e., 20-40 cm) than CAM plants. To evaluate the effect of deeper grass root zones, I allow the root depth of grasses to differ from that of CAM plants (i.e., $Z_{l}$ ) and investigate the model's sensitivity to changes in this parameter. The sensitivity of this model with respect to changes of rainfall regime is also evaluated. The results of this sensitivity analysis, which are detailed in Supplementary Material, are generally consistent with those presented in the main text.

\subsection{Results}

I first focus on the case of a plant community with a high degree of root overlap between trees and CAM plants; in these conditions trees with very shallow roots (i.e., $20 \mathrm{~cm}$ ) cannot perform hydraulic redistribution. A high degree of root overlap leads to a relatively strong competition 
for soil water resources between trees and CAM plants both in arid (Figure $2 a)\left(\lambda=0.2 d^{-1}\right.$ and $h=5 \mathrm{~mm}$ ) and semiarid environments (Figure $2 \mathrm{~b})\left(\lambda=0.2 \mathrm{~d}^{-1}\right.$ and $\left.h=10 \mathrm{~mm}\right)$; this fact is evidenced by a lower transpiration of CAM plants in tree-CAM (T-C) associations than by themselves.
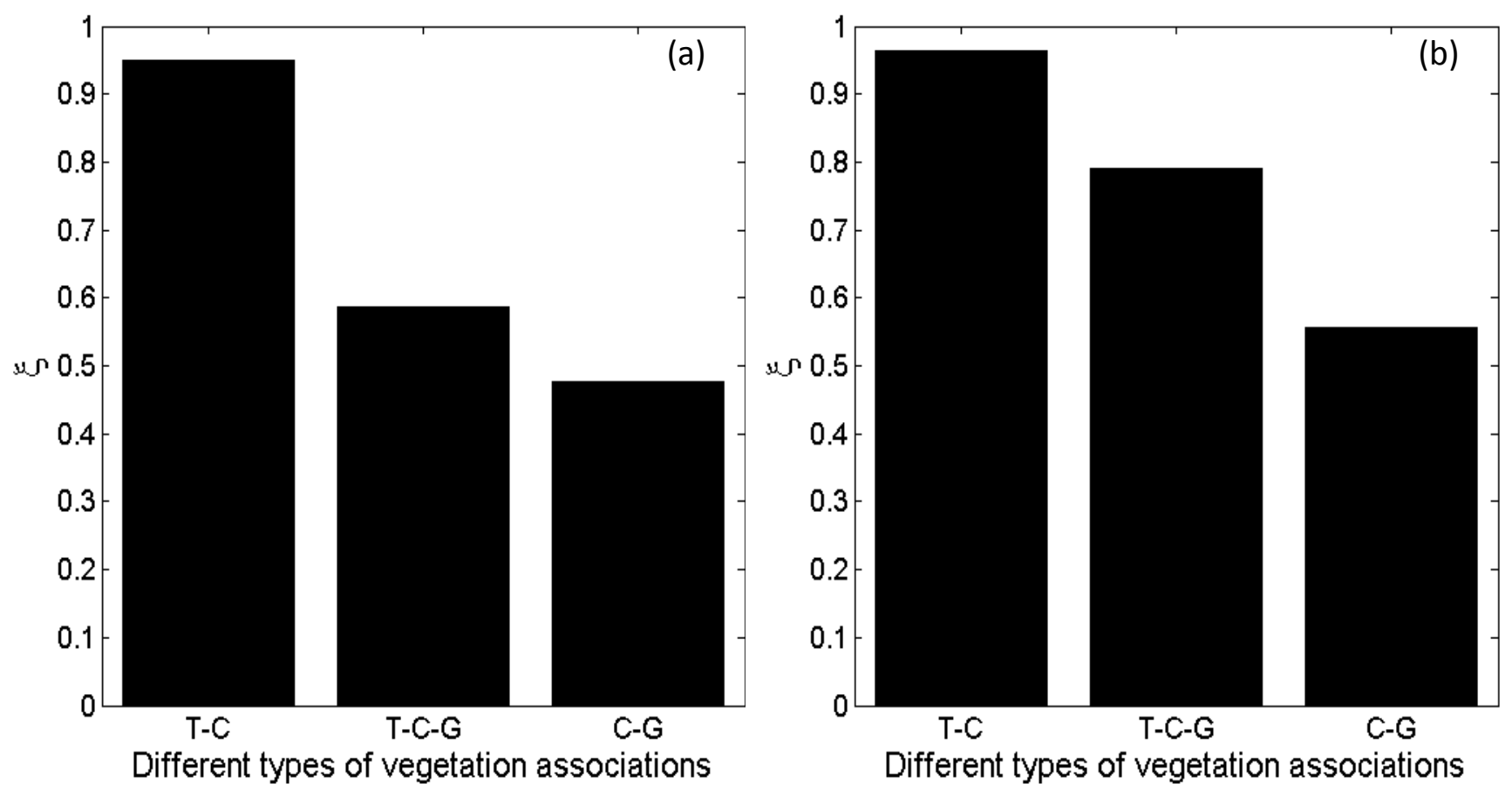

Figure 8-2: Transpiration ratio ( $\xi$ ) of CAM plants between CAM associations and CAM alone for arid $\left(\lambda=0.2 \mathrm{~d}^{-1}\right.$ and $\left.\mathrm{h}=5 \mathrm{~mm}\right)$ (a) and semiarid $\lambda=0.2 \mathrm{~d}^{-1}$ and $\left.\mathrm{h}=10 \mathrm{~mm}\right)$ (b) environment in loamy sand in the case of a high degree of root overlap between trees and CAM plants. Parameters: the depth of shallow soil layer, $Z_{1}=10 \mathrm{~cm}$; the depth of deep soil layer, $Z_{2}=10 \mathrm{~cm}$; grass cover in arid and semiarid environment, $f_{g}=70 \%$; root allocation into the deep soil layer, $r_{2} / r_{1}=0.2$.

This result can be explained by the high rate of water uptake from the shallow soil layer by trees and thus the high water losses $\left(\mathrm{ET}_{1}\right)$ from the shallow soil and the lower soil water availability (Figure 3a, b). 

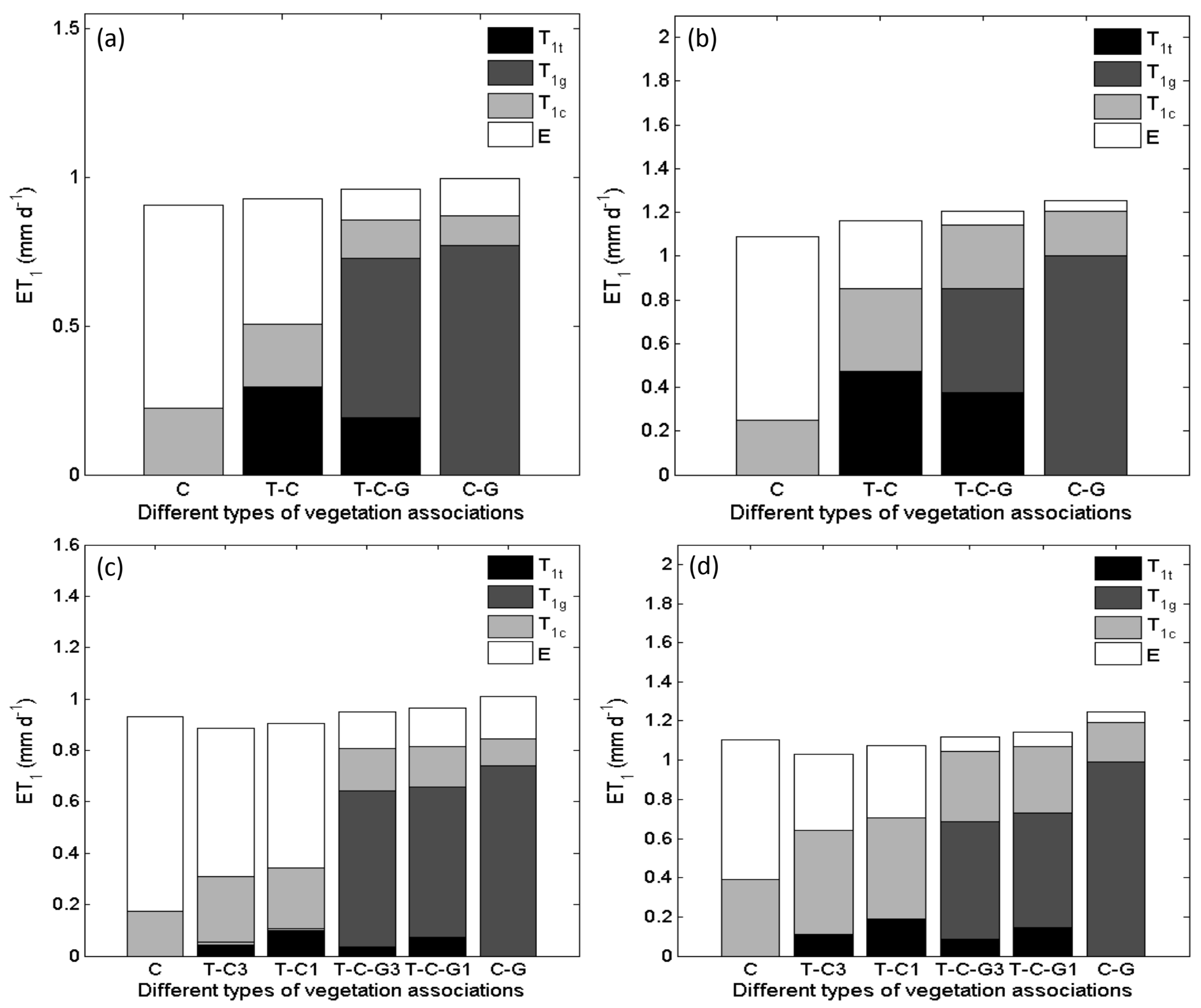

Figure 8-3: Actual evapotranspiration components in the shallow soil layer for CAM plants alone (C), tree-CAM (T-C), tree-CAM-grass (T-C-G), and CAM-grass $(\mathrm{C}-\mathrm{G})$ associations for arid $(\lambda=$ $0.2 \mathrm{~d}^{-1}$ and $\left.\mathrm{h}=5 \mathrm{~mm}\right)$ (a) and semiarid $\left(\lambda=0.2 \mathrm{~d}^{-1}\right.$ and $\left.\mathrm{h}=10 \mathrm{~mm}\right)(\mathrm{b})$ environment in loamy sand in the case of a high degree of root overlap between trees and CAM plants. $T_{1 t}$ refers to the transpiration by trees, $T_{1 \mathrm{~g}}$ refers to the transpiration by grasses, $\mathrm{T}_{1 \mathrm{c}}$ refers to the transpiration by CAM plants, and E refers to evaporation from the soil surface. Parameters: the same as Figure 3 $(a, b) .(c, d)$ Actual evapotranspiration components in the shallow soil layer for CAM plants alone (C), tree-CAM (T-C), tree-CAM-grass (T-C-G), and CAM-grass (C-G) associations for arid $\left(\lambda=0.2 \mathrm{~d}^{-1}\right.$ and $\left.\mathrm{h}=5 \mathrm{~mm}\right)(\mathrm{c})$ and semiarid $\left(\lambda=0.2 \mathrm{~d}^{-1}\right.$ and $\left.\mathrm{h}=10 \mathrm{~mm}\right)(\mathrm{d})$ environment, in loamy sand in the case of a low to moderate root overlap between trees and CAM plants. $\mathrm{r}_{2} / \mathrm{r}_{1}$ : root allocation into the deep soil layer. The number " 3 " means $r_{2} / r_{1}=3$ while the number " 1 " means $\mathrm{r}_{2} / \mathrm{r}_{1}=1$. Parameters: depth of shallow soil layer, $\mathrm{Z}_{1}=10 \mathrm{~cm}$; depth of deep soil layer, $\mathrm{Z}_{2}$ $=30 \mathrm{~cm}$; grass cover in arid and semiarid environment, $\mathrm{f}_{\mathrm{g}}=70 \%$. 
Likewise, grasses exert a higher competition on CAM plants than trees in both arid (Figure 2a) and semiarid environments (Figure 2b). Interestingly, transpiration of CAM plants in the treeCAM-grass associations (T-C-G) is higher than that in the CAM-grass associations both in arid (Figure 2a) and semiarid environments (Figure 2b), which indicates that trees facilitate CAM plants. This facilitation of trees on CAM plants in tree-CAM-grass associations results from a substantial reduction in grass transpiration (Figure 3a, b). Overall, these results indicate that trees indirectly facilitate CAM plants by significantly reducing grass transpiration.

I now focus on the case in which woody plants have deeper roots and can thus perform hydraulic redistribution. In arid environments $\left(\lambda=0.2 \mathrm{~d}^{-1}\right.$ and $\left.h=5 \mathrm{~mm}\right)$, drainage $\left(\mathrm{D}_{1}\right)$ from the shallow to the deep soil layer is overall small and therefore the deep soil layer is often drier than the shallow soil. Thus hydraulic redistribution is often in the form of hydraulic descent (i.e., downward) performed by trees (Figure 4a). In contrast, trees perform hydraulic lift in semiarid environments ( $\lambda=0.2 \mathrm{~d}^{-1}$ and $h=10 \mathrm{~mm}$ ), where drainage intensity is sufficient to maintain higher levels of soil moisture in the deep than in the shallow soil (Figure 4b); a low allocation of roots to the deep soil layer (i.e., high $r_{1} / r_{2}$ ) increases water usage in the shallow soil and thus enhances hydraulic lift (Figure 4b). Inclusion of grasses into tree-CAM associations also increases water usage in the shallow soil, thus reducing hydraulic descent in arid environments (Figure 4a) and increasing hydraulic lift in semiarid environments (Figure 4b). Regardless of the effects of hydraulic redistribution, direct facilitation of trees on CAM plants occurs in situations of a low to moderate root overlap between trees and CAM plants and this effect is weaker in arid (Figure 4c) than in semiarid environments (Figure 4d). Hydraulic descent reduces (Figure 4a and Figure $4 \mathrm{c}$ ) while hydraulic lift increases the direct facilitation of CAM plants by trees (Figure $4 \mathrm{~b}$ and Figure 4d). Interestingly, a high rate of hydraulic lift can lead to a higher transpiration of 
CAM plants in tree-CAM-grass associations (T-C-G) with respect to the case of CAM plants alone (C) (Figure 4d), which indicates that CAM plants may prefer to establish themselves under canopies of trees even in the presence of grass competition.
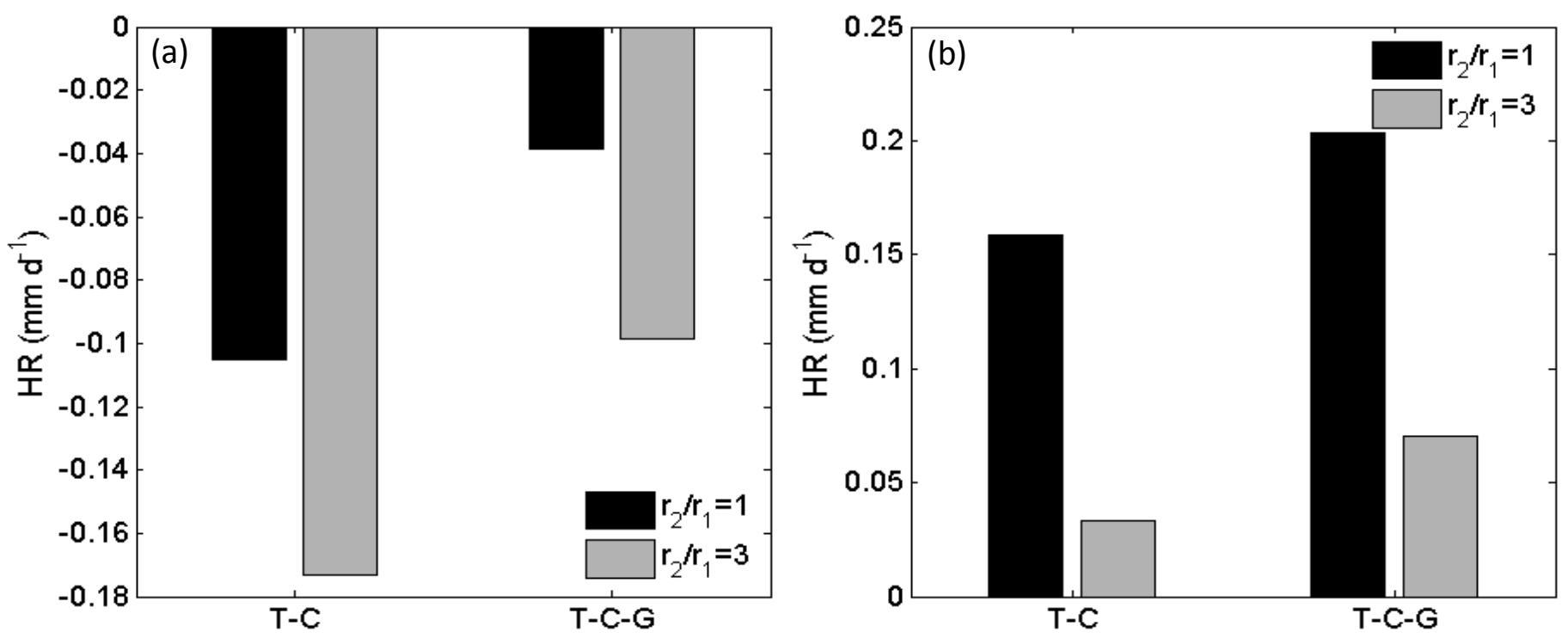

Different types of vegetation associations
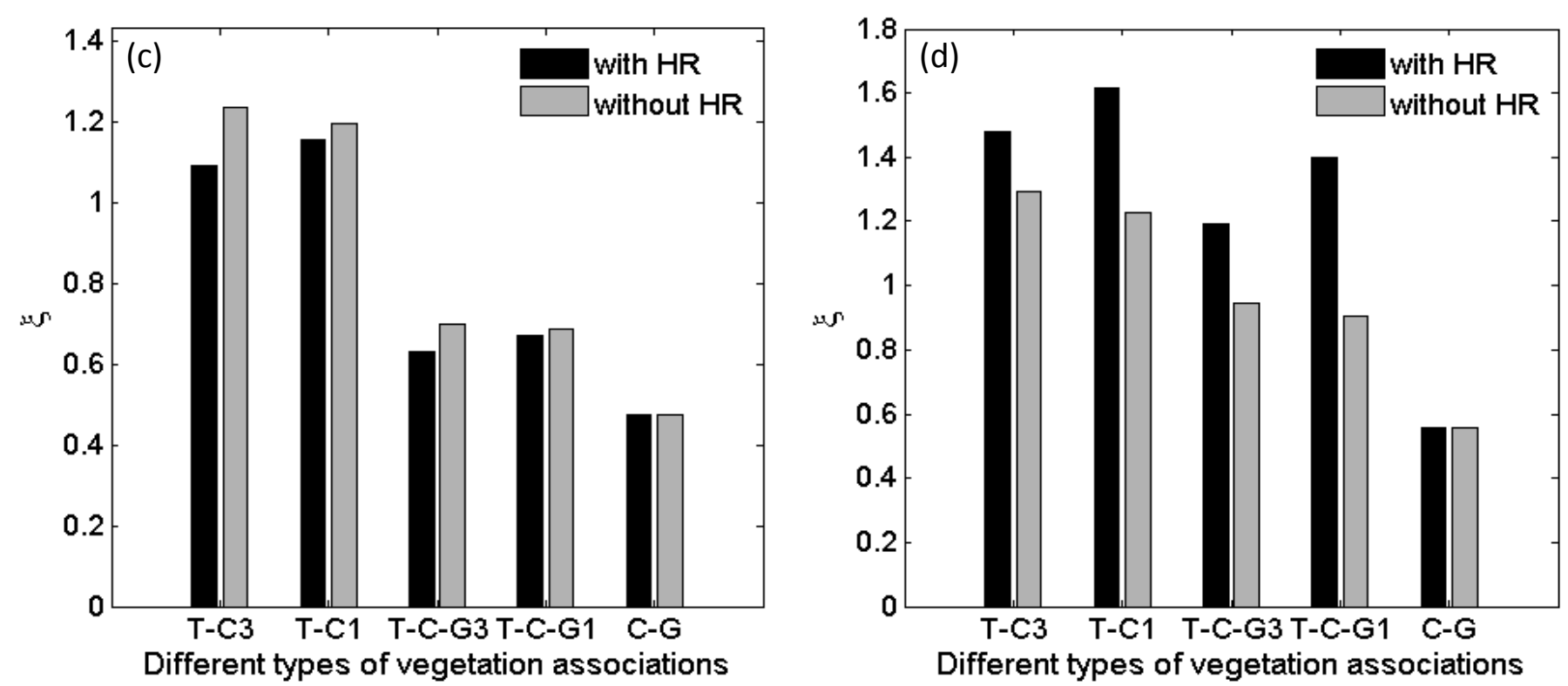

Figure 8-4: Hydraulic redistribution (HR) by trees in tree-Cam (T-C) and tree-CAM-grass (T-CG) associations for arid $\left(\lambda=0.2 \mathrm{~d}^{-1}\right.$ and $\left.\mathrm{h}=5 \mathrm{~mm}\right)$ (a) and semiarid $\left(\lambda=0.2 \mathrm{~d}^{-1}\right.$ and $\left.\mathrm{h}=10 \mathrm{~mm}\right)$ (b) environments. (c, d) Transpiration ratio $(\xi)$ of CAM plants between CAM associations and CAM alone for arid $\left(\lambda=0.2 \mathrm{~d}^{-1}\right.$ and $\left.\mathrm{h}=5 \mathrm{~mm}\right)(\mathrm{d})$ and semiarid $\left(\lambda=0.2 \mathrm{~d}^{-1}\right.$ and $\left.\mathrm{h}=10 \mathrm{~mm}\right)(\mathrm{d})$ environments. All panels refer to the case of loamy sand and low to moderate root overlap between trees and CAM plants. Parameters: the same as Figure 3 (c, d). 
The indirect facilitation of trees on CAM plants occurs because trees substantially reduce grass transpiration in tree-CAM-grass associations (T-C-G) as compared to CAM-grass associations (C-G), especially in semiarid environments (Figure 3c, d). Overall, this leads to a higher transpiration of CAM plants when they are in tree-CAM-grass associations (T-C-G) than in CAM-grass associations (C-G) (Figure 4c, d). Thus, I conclude that direct facilitation of CAM plants by trees occurs simultaneously with the indirect effect in situations of a low-to-moderate root overlap between trees and CAM plants.

Transpiration of CAM plants in tree-CAM-grass associations (T-C-G) is lower than that in tree-CAM associations (T-C) in both arid (Figure 4c and Figure 5a) and semiarid environments (Figure $4 d$ and Figure 5b) regardless of the effects of hydraulic redistribution. A higher rate of hydraulic lift by trees in tree-CAM-grass associations reduces the competitive effects of grasses on CAM plants, as indicated by an increase of transpiration ratio of CAM plants between treeCAM-grass associations (T-C-G) and CAM-grass (C-G) (Figure 4d). However, reduction in hydraulic descent and the increase in hydraulic lift cannot outweigh the increase of shallow soil moisture depletion by grass transpiration (competitive effect of grasses) (Figure 5c, d). This explains why an increase in grass cover reduces transpiration ratio of CAM plants between treeCAM-grass associations (T-C-G) and tree-CAM associations (T-C) (Figure 5a, b). Overall, these results indicate that the introduction of grasses in tree-CAM associations still exerts a competitive effect on CAM plants in presence of a relatively high hydraulic lift rate. In other words, the addition of grasses cannot indirectly facilitate CAM plants through the mechanism of hydraulic lift. 

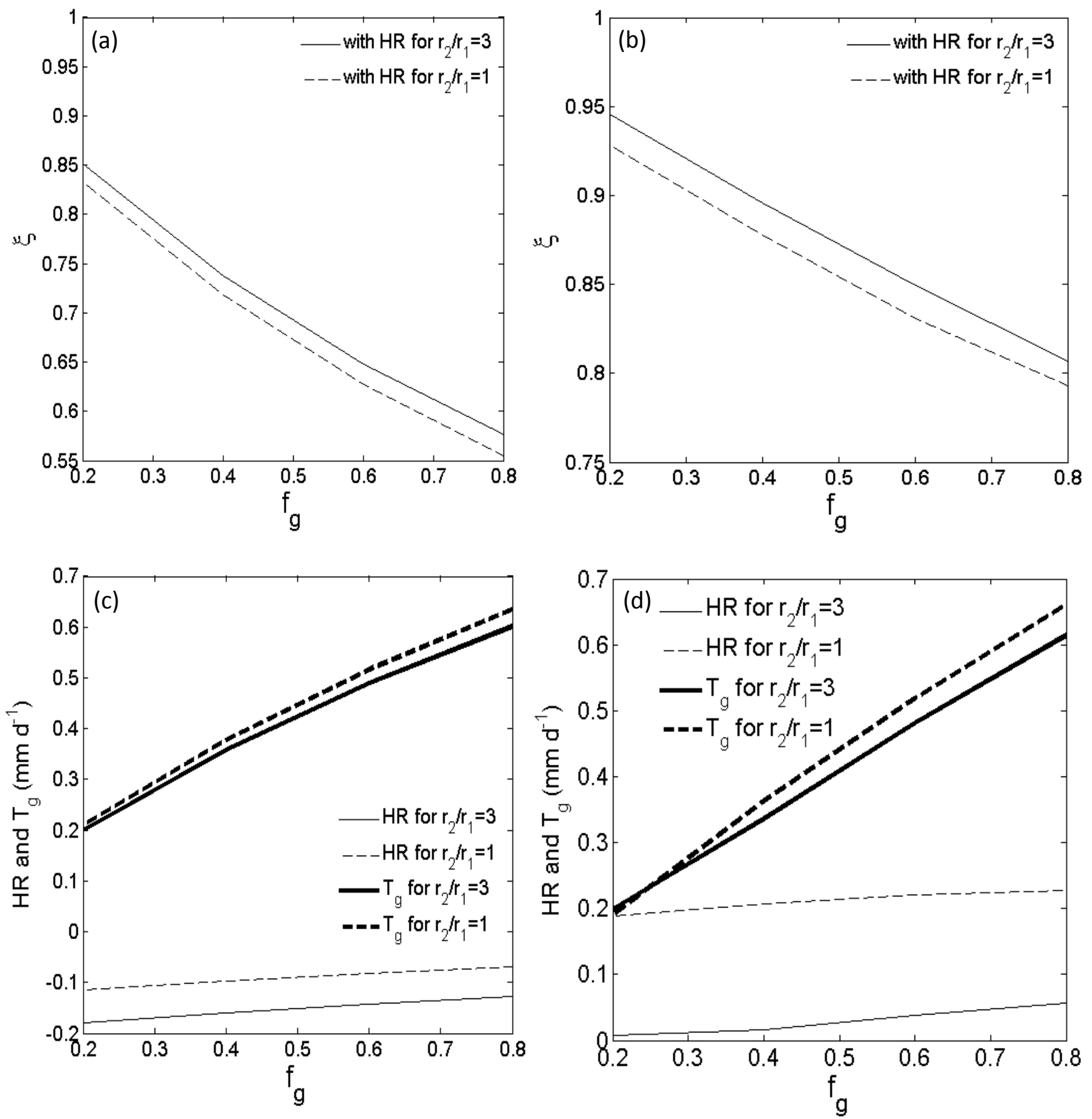

Figure 8-5: Transpiration ratio $(\xi)$ of CAM plants between tree-CAM-grass association (T-C-G) and tree-CAM association (T-C) for arid $\left(\lambda=0.2 \mathrm{~d}^{-1}\right.$ and $\left.\mathrm{h}=5 \mathrm{~mm}\right)$ (a) and semiarid $\left(\lambda=0.2 \mathrm{~d}^{-1}\right)$ and $\mathrm{h}=10 \mathrm{~mm}$ ) (b) environments in loamy sand as affected by grass cover $\left(\mathrm{f}_{\mathrm{g}}\right)$. (c, d) Hydraulic redistribution $(H R)$ and grass transpiration $\left(\mathrm{T}_{\mathrm{g}}\right)$ in tree-CAM-grass $(\mathrm{T}-\mathrm{C}-\mathrm{G})$ association in loamy sand as affected by grass cover $\left(\mathrm{f}_{\mathrm{g}}\right)$ for arid (c) and semiarid (d) environments. Parameters: the depth of shallow soil layer, $Z_{1}=10 \mathrm{~cm}$; the depth of deep soil layer, $Z_{2}=30 \mathrm{~cm}$. 


\subsection{Discussion}

Our study evaluates conditions that could lead to indirect facilitation in dryland vegetation. Particularly, I focus on the case of CAM plants whose direct facilitation by woody plants has been widely documented (Withgott, 2000; Castillo and Valiente-Banuet, 2010; Cares et al., 2013). I developed a model to quantify transpiration of CAM plants in CAM plants alone (C), CAM-grass (C-G), tree-CAM (T-C), and tree-CAM-grass (T-C-G) associations, at seasonal-toannual timescales. A comparison of transpiration of CAM plants in these communities allows us to investigate the direct and indirect facilitation in tree-CAM-grass (T-C-G) associations. The role of hydraulic redistribution is accounted for by coupling soil moisture dynamics in a shallow soil layer and the underlying soil (Ryel et al., 2002; Lee et al., 2005; Yu and D'Odorico, 2014b).

My study shows that woody plants having a high degree of root overlap with CAM plants indirectly facilitate CAM plants in the access to soil water resources (Figure 1a; Figure 2a, b). The indirect facilitation occurs because woody plants significantly reduce grass transpiration through solar radiation reduction (Figure 3a, b) and thus reduce the competition of grasses on CAM plants in the access to soil water resources (Levin, 1999; Brooker et al., 2008). These results are consistent with other studies. For example, Siemann and Rogers (2003) found that canopies of alien Chinese tallow tree (Sapium sebiferum) reduce the competitive interaction of grasses and thus indirectly facilitate the growth of tree seedlings in shaded conditions. Kunstler et al (2006) indicated that shade from shrub canopies indirectly facilitates Fagus survival by limiting herb competition for access to soil water resources. Other possible mechanisms of grass suppression by trees include allelopathy (Knipe and Herbel, 1966; Callaway, 2007; Ehlers et al., 2014). In contrast, other studies indicate that the indirect positive effect can be outweighed by the direct negative effect (Pagès et al., 2003 and Pagès and Michalet, 2003), thus precluding the 
occurrence of indirect facilitation (Levin, 1999; Brooker et al., 2008). In my study, CAM plants are thought to be shade tolerant because of their photosynthetic plasticity and acclimation to shade, as confirmed by experimental evidence (Medina et al., 1986; Fetene et al., 1990; Ceusters et al., 2011).

My study also shows that woody plants having a low to moderate root overlap with CAM plants have a direct facilitation effect on CAM plants along with an indirect facilitation effect (Figure 1b; Figure 4c, d). The direct facilitation effect results from a substantial reduction of evaporation from the soil surface (shade effect) (Figure 3c, d), consistent with other studies (Ludwig et al., 2004; D'Odorico et al., 2008; Dohn et al., 2013; Moustakas et al., 2013). Experimental evidence confirming the direct facilitation effects of woody plants on CAM plants are extensive (Withgott, 2000; Castillo and Valiente-Banuet, 2010; Cares et al., 2013). The simultaneous occurrence of direct and indirect facilitation was also suggested by other studies. Miller (1994) found that indirect effect is often confounded by the simultaneous occurrence of direct effect. Siemann and Rogers (2003) documented the occurrence of direct facilitation via nitrogen and indirect facilitation via light reduction in tree-tree seedling-grass associations in Texas, USA.

Woody plants with relatively deep roots and a low to moderate root overlap with CAM plants can perform hydraulic redistribution (Figure 4a, b), which may play a role in the direct and indirect facilitation in the tree-CAM-grass (T-C-G) associations. Past studies suggest that hydraulic lift can contribute to the facilitation of understory plants by trees (Riginos et al., 2009; Dohn et al., 2013; Moustakas et al., 2013). My study confirms the weakening of direct facilitation of CAM plants by trees with hydraulic descent (Figure 4c) and the enhancement of direct facilitation when hydraulic lift occurs (Figure 4d). Moreover, I found that hydraulic lift 
increases transpiration of CAM plants in tree-CAM-grass (T-C-G) associations with respect to the case with CAM plants alone, in contrast to the situation without hydraulic lift (Figure 4d). Therefore, hydraulic lift may contribute to explain the preference of CAM plants to establish and grow under tree canopies even in presence of grass competition rather than bare soils. Unlike the case of direct facilitation, hydraulic lift may reduce the indirect facilitation of CAM plants by trees because it favors grasses at the expense of trees (Richards and Caldwell, 1987; Zou et al., 2005; Brooks et al., 2006; Yu and D'Odorico, 2014b), thereby increasing the competitive effects of grasses on CAM plants. This effect depends on how CAM plants and grasses compete for the access to hydraulically lifted water. CAM plants transpire and photosynthesize at night when trees perform hydraulic lift, while neither trees nor grasses transpire for photosynthesis (Lüttge, 2004; Ogburn and Edwards, 2010). Thus, it has been suggested that CAM plants should benefit more than grasses from hydraulic lift (Yoder and Nowak, 1999). By comparison, hydraulic descent suppressing grass growth increases the indirect facilitation of trees on CAM plants (Burgess et al., 2001; Hultine et al., 2004).

Past studies have largely ignored whether inclusion of grasses in tree-CAM associations can indirectly facilitate CAM plants through the mechanism of hydraulic lift (Figure 1c). Yu and D'Odorico (2014b) found that a high rate of transpiration by grasses in the shallow soil layer can promote the occurrence of hydraulic lift. Thus, the indirect facilitation of CAM plants by grasses will occur if the benefits from hydraulic lift outweigh the competitive effects of grasses on CAM plants (Levin, 1999; Brooker et al., 2008). My study shows that the competitive effects of grasses on CAM plants outweigh the hydraulic lift effect (Figure 5d), thereby leading to a lower transpiration of CAM plants in tree-CAM-grass associations (T-C-G) than without grasses (i.e., T-C) (Figure 4d). Therefore, transpiration ratio of CAM plants between tree-CAM-grass 
association (T-C-G) and tree-CAM association (T-C) decreases with increasing grass cover even in cases with relatively high rates of hydraulic lift (Figure 5b). In fact, conditions that maximize hydraulic lift (high hydraulic conductivity, relatively small leaf area index, and high tree root allocation in the shallow soil layer) are associated with strong grass competition with CAM plants. However, the ability of CAM plants to benefit from hydraulic lift cannot be ignored because of their preferential access to hydraulically lifted water (Yoder and Nowak, 1999; Lüttge, 2004; Ogburn and Edwards, 2010). Overall, this model based study provides the first analysis of the direct and indirect facilitation in the tree-CAM-grass (T-C-G) associations. Experimental evidence is needed to further support these results.

In summary, this study found that a high degree of root overlap favors competition between trees and CAM plants for soil water resources, but trees indirectly facilitate CAM plants by significantly reducing grass transpiration in shaded conditions. In conditions with a low to moderate root overlap, the indirect effect is confounded by the simultaneous occurrence of the direct effect. The increase of hydraulic lift with inclusion of grasses in tree-CAM association is not sufficient to outweigh the competitive effects of grasses on CAM plants, thus precluding the indirect facilitation of grasses on CAM plants through hydraulic lift. 


\section{Conclusions and future developments}

The series of studies presented in this dissertation are motivated by the need to understand the impacts of major environmental change drivers on plant communities in drylands. Two major dryland vegetation changes associated with woody plant-grass interactions and expansion of CAM plants are investigated using mechanistic modelling frameworks integrated with field or remote sensing data and/or experiments in greenhouse facilities. In what follows, the main conclusions in each chapter are summarized and future developments are discussed.

Chapter 2 presents an ecohydrological framework to show that the lateral root spread and soil water and light limitations can allow woody plant encroachment to substantially suppress grass production even in the absence of grazers. The ability of shrubs to displace grasses allows for the emergence of a grass-fire feedback, whereby the loss of grasses reduces fire pressure on woody plants and thus further sustains woody plant encroachment. Such a feedback may explain the existence of bistable vegetation dynamics in transition zones between grasslands and shurblands.

Chapter 3 shows that grass invasions and climate change (increased rainfall fluctuations) act in concert to induce the ecosystem transition from shurblands to unvegetated state. This finding is achieved by developing a stochastic process-based model of vegetation-resource dynamics. This study suggests that changes in the variance of annual precipitation are also important when shifts in vegetation composition (e.g., species invasions) result in changes in vegetation susceptibility to climatic variability. In the presence of random climate fluctuations ecosystems can display steady states that differ from those that would exist under a constant climate or with a climate trend.

Chapter 4 develops a mechanistic model to investigate a new mechanism of woody plant-grass coexistence which are usually explained by invoking niche separation or disturbances. It shows that hydraulic lift favors grasses, which scavenge the water lifted by woody plants and thus 
contribute to woody plant-grass coexistence. Hydraulic lift expands (at the expenses of woodlands) the range of environmental conditions in which savannas are stable. The mechanistic model is further adapted to investigate the response of tree-grass composition to increasing interannual rainfall variability along the Kalahari rainfall gradient. It shows the way tree-grass composition responds to increased interannual variation in precipitation would depend on key traits of trees and grasses (i.e., growth rate and root depth) that determine their ability to take advantage of the windows of opportunity offered by periods with higher soil moisture.

Chapter 5 develops growth chamber experiments to show that under $\mathrm{CO}_{2}$ enrichment and drought conditions Cylindropuntia imbricata (a constitute CAM plant) outcompetes Bouteloua eriopoda $\left(\mathrm{C}_{4}\right.$ grass), with which it coexists in semiarid ecosystems across the southwestern United States. Combined with a meta-analysis examining multiple plant families and showing a positive response of CAM photosynthesis and productivity to $\mathrm{CO}_{2}$ enrichment, this study suggests that under drought and elevated $\mathrm{CO}_{2}$ concentrations projected with climate change the dominance of CAM plants is likely to keep increasing in semiarid ecosystems.

Chapter 6 presents greenhouse experiments to show that drought and nitrogen deposition, which are predicted to increase in the near future, are important divers of expansion of facultative CAM plants such as $M$. crystallinum. In well-watered conditions, however, B. mollis ( $\mathrm{C}_{3}$ grasses), with which $M$. crystallinum interacts in the coastal grasslands of California is in competitive advantage with respect to $M$. crystallinum; the strong competition from $B$. mollis can restrict the ability of $M$. crystallinum to perform nocturnal carboxylation, thereby limiting its photosynthetic plasticity to adapt to biological stress.

Chapter 7 develops mechanistic models to show the potential ability of deep-rooted CAM plants in CAM-grass associations to perform hydraulic lift at a higher rate than trees in tree-grass 
associations in a relatively wet environment. The ability of CAM plants to perform hydraulic redistribution at a higher rate may have different effects on the surrounding plant community relative to hydraulic effects for plants with $\mathrm{C}_{3}$ or $\mathrm{C}_{4}$ photosynthetic pathways (i.e., diurnal transpiration). By comparison, a high rate of hydraulic descent performed by trees in tree-CAM associations could even turn the facilitation of CAM plants by woody plants into competition. This may change understanding of the role of root depth in niche differentiation because deep root development, which is usually thought to reduce competition and thus promote coexistence with shallow-rooted plants, could also favor competition through the mechanism of hydraulic descent.

Chapter 8 develops a mechanistic model to investigate the complex interactions involving three functional groups (i.e., woody plants, grasses, CAM plants). Trees directly facilitate CAM plants in access to soil water resources because of shade effects in situations of a low-to-moderate root overlap. Moreover, trees could suppress grass transpiration through solar radiation reduction and reduce the competitive effect of grasses on CAM plants in access to soil water resources, thus indirectly facilitating CAM plants. These results suggest that interactions with other species and the relationship with other ongoing changes in plant composition (e.g., woody plant encroachment) could contribute to the expansion of CAM plants, in addition to other drivers such as climate change (see chapters 5 and 6).

In summary, research in ecology in the last few decades has investigated the response of $\mathrm{C}_{3}$ and $\mathrm{C}_{4}$ plants to global environment change. Many novel aspects of this dissertation clarify key ecohydrological controls of woody plant-grass interactions. Unlike changes in woody plant-grass dominance, the expansion of CAM plants or more broadly shifts in the competitive relationship between CAM plants and other functional groups (i.e., $\mathrm{C}_{3}$ and $\mathrm{C}_{4}$ plants) under global environmental change are under investigated. The expansion of CAM plants in drylands could 
bring new opportunities for the use of marginal lands for food and biofuel production, and therefore improve human adaptation to climate change through provision of bioenergy, greater food security, and carbon sequestration. Understanding the potential shift of the competitive relationships between CAM plants and other functional groups is crucial to the understanding of ecosystem resilience and productivity, and the provision of ecosystem services in arid landscapes under global environmental change. While this dissertation investigates the competitive advantages of $\mathrm{CAM}$ plants over $\mathrm{C}_{3}$ and $\mathrm{C}_{4}$ grasses under changes in atmospheric $\mathrm{CO}_{2}$, drought and $\mathrm{N}$ deposition, future studies need to investigate the effects of other major global environmental drivers such as climate warming and changes in rainfall variability. 


\section{Appendices}

A1 An ecohydrological framework for grass displacement by woody plants in savannas

$\underline{\text { Supplementary information }}$

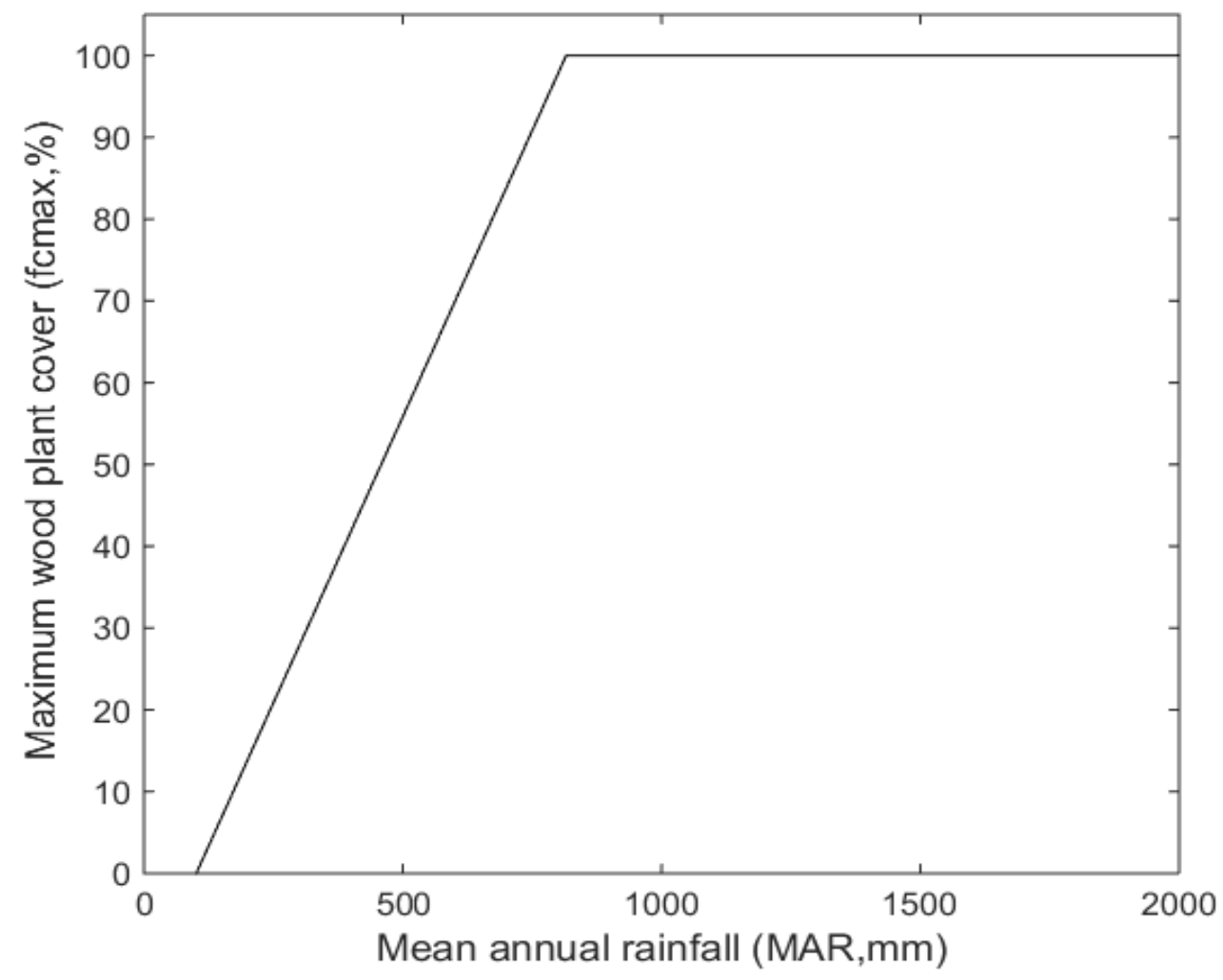

Supplementary Figure 2-1: The relationship between maximum woody plant cover (fcmax) and mean annual rainfall (MAP) in African savannas (modified from Sankaran et al., 2005). 


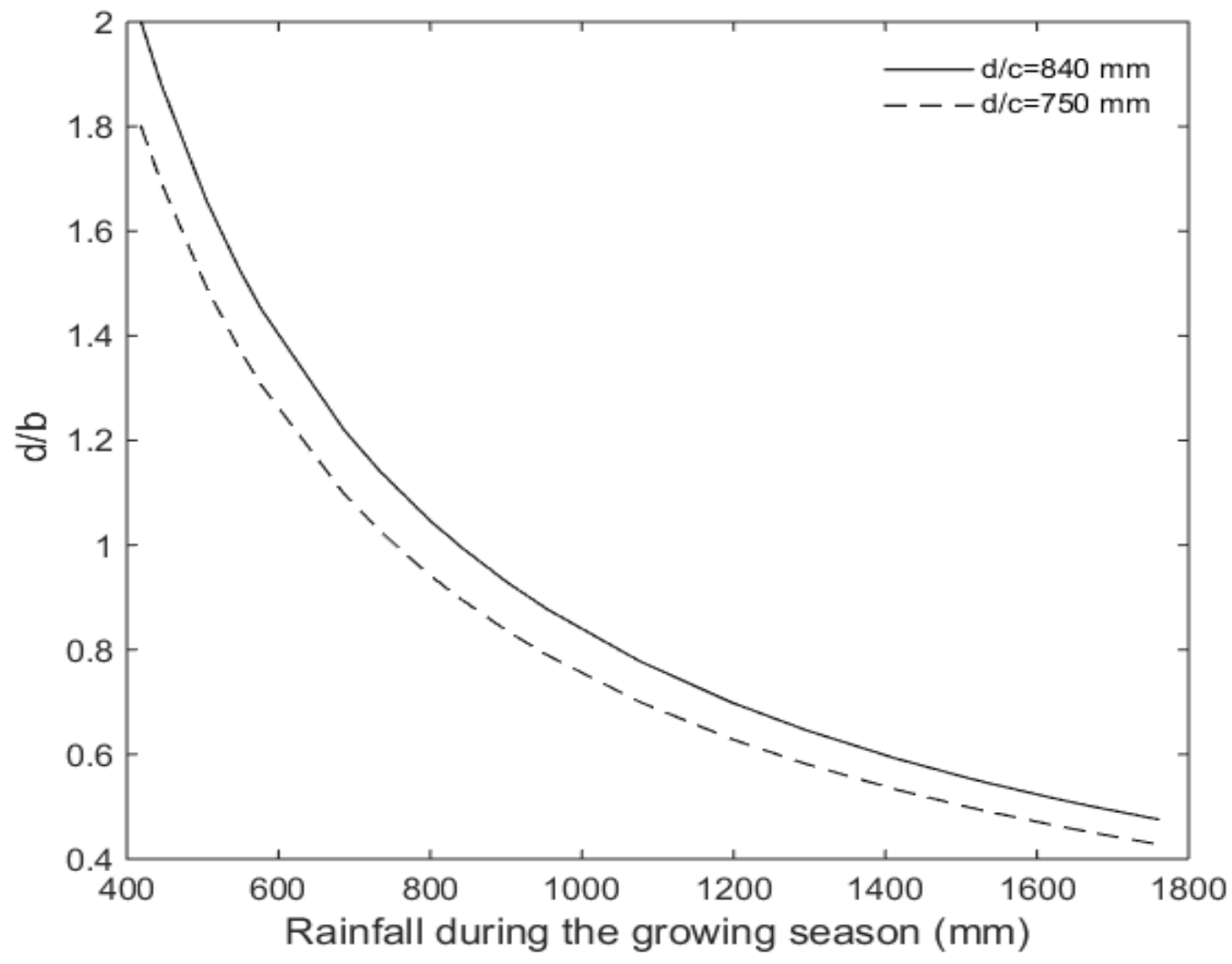

Supplementary Figure 2-2: The relationship between ratio of fire-induced mortality to growth rate of woody plants $(\mathrm{d} / \mathrm{b})$ and rainfall during the growing season. 


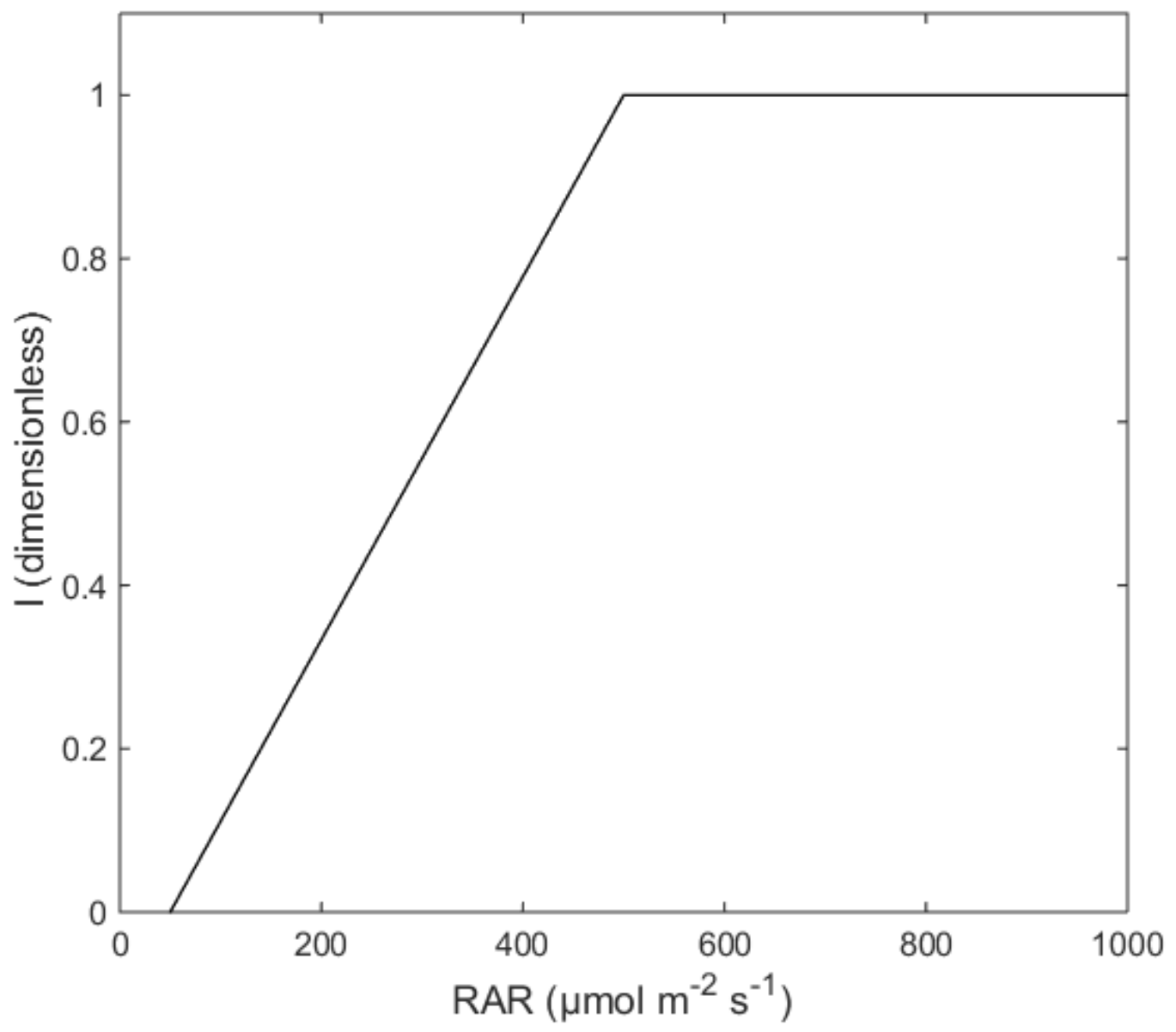

Supplementary Figure 2-3: Dependence between fire frequency and fuel load (i.e., grass cover) at Kruger National Park, South Africa (experiment data from van Wilgen et al., 2000). 
Supplementary Table 2-1: Location, elevation (he), mean annual rainfall (MAP), mean atmospheric temperature $\left(\mathrm{Te}_{\mathrm{a}}\right)$ in Tshane and Mongu in Southern African.

\begin{tabular}{ccccc}
\hline Site & Location & he $(\mathrm{m})$ & MAR $(\mathrm{mm})$ & $\mathrm{Te}_{a}(\mathrm{~K})$ \\
\hline Tshane & $24.164^{\circ} \mathrm{S}, 21.893^{\circ} \mathrm{E}$ & 1118 & 365 & 25.3 \\
Mongu & $15.438^{\circ} \mathrm{S}, 23.253^{\circ} \mathrm{E}$ & 1052 & 879 & 21.1 \\
\hline
\end{tabular}


Supplementary Table 2-2: Parameters, parameter values, and reference sources used in the coupled energy and water balance model

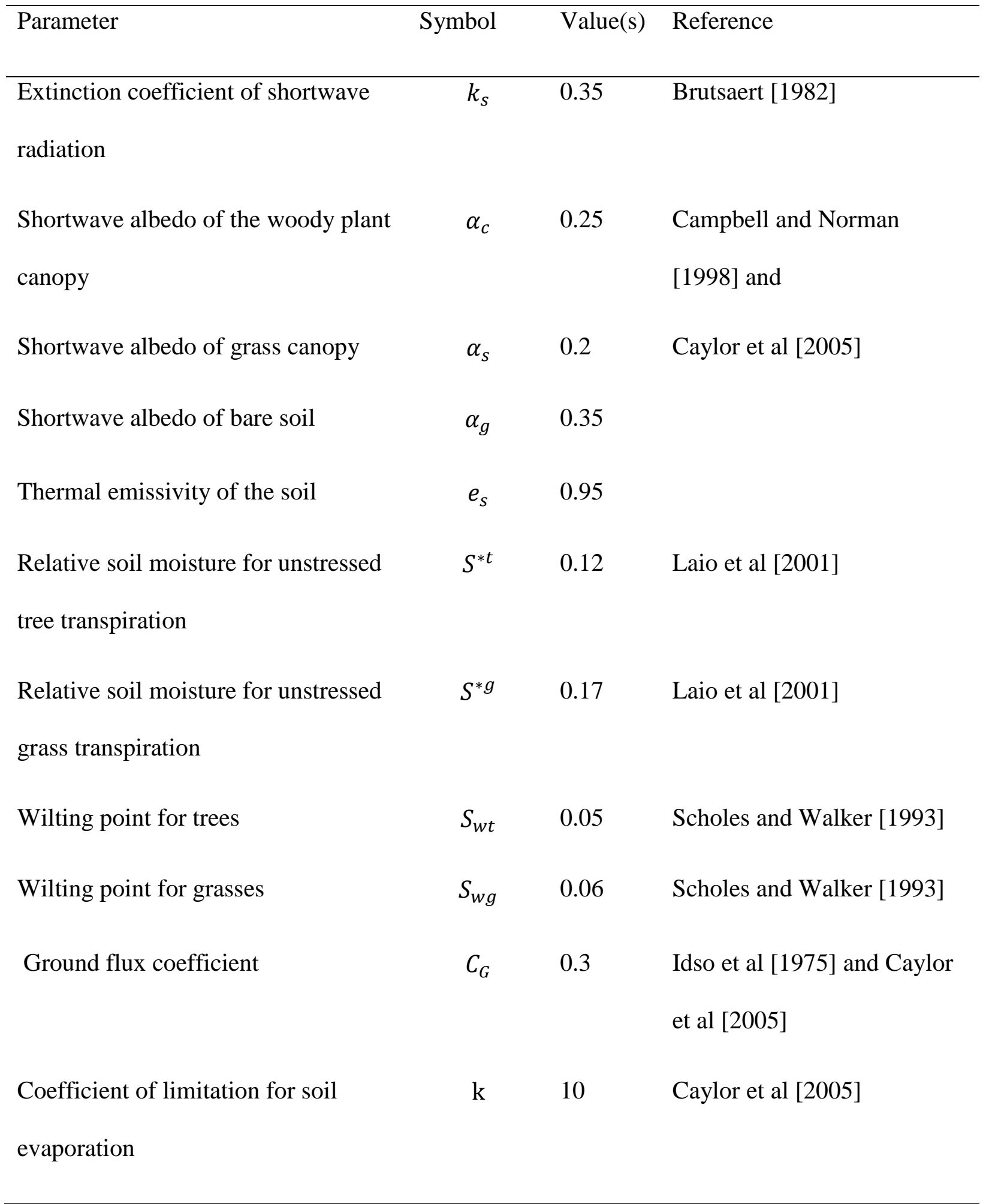




\begin{tabular}{|c|c|c|c|}
\hline Field capacity & $S_{f c}$ & 0.4 & \\
\hline \multirow[t]{2}{*}{ Effective rooting depth } & $Z_{R}$ & 1000 & \\
\hline & & $\mathrm{mm}$ & \\
\hline Soil porosity & $\mathrm{n}$ & 0.4 & Wang et al [2007] \\
\hline Empirical coefficient & $\mathrm{a} 1$ & -2.1154 & Caylor et al [2005] \\
\hline \multirow[t]{5}{*}{ for calculation of soil temperature } & $\mathrm{a} 2$ & 0.0108 & Caylor et al [2005] \\
\hline & a3 & 1.0029 & Caylor et al [2005] \\
\hline & b1 & 2.085 & Caylor et al [2005] \\
\hline & $\mathrm{b} 2$ & 0.0005 & Caylor et al [2005] \\
\hline & b3 & 0.0425 & Caylor et al [2005] \\
\hline
\end{tabular}




\section{A2 Potential of grass invasions in fireproof desert shrublands to create novel ecosystem states under variable climate}

Supplementary information

Supplementary Table 3-1: A summary of evidence of a high sensitivity of perennial grass to drought

\begin{tabular}{|c|c|c|c|}
\hline Region & Perennial grass investigated & Main findings & References \\
\hline Southern New & Bouteloua eriopoda & Drought can rapidly reduce grass & Buffington and \\
\hline \multirow[t]{3}{*}{ Mexico, USA } & & cover across space; drought leads & Herbel (1965); \\
\hline & & to the loss of perennial grasses & Herbel et al. (1972); \\
\hline & & during woody plant expansion & Yao et al. (2006) \\
\hline Southern Arizona, & Eragrostis lehmanniana, & Mean annual rainfall below the & McClaran and \\
\hline \multirow[t]{3}{*}{ USA } & Bouteloua rothrockii, & threshold of $350 \mathrm{~mm}$ greatly limit & Angell (2006) \\
\hline & Digitaria californica, & grass cover & \\
\hline & Muhlenbergia porteri & & \\
\hline Southern Arizona, & Eragrostis lehmanniana, & Low rainfall is an more important & Mashiri et al. (2008) \\
\hline \multirow[t]{3}{*}{ USA } & Bouteloua rothrockii, & factor limiting perennial grass & \\
\hline & Digitaria californica, , & cover than grazing & \\
\hline & Aristida spp. & & \\
\hline College Station, & Schizachyrium scoparium & Drought leads to the greatest & Volder et al. (2010) \\
\hline \multirow[t]{2}{*}{ Texas, USA } & & reduction in leaf-level net & \\
\hline & & photosynthesis of S.scoparium & \\
\hline
\end{tabular}


comapred with shurbs (i.e.,

Quercus stellata, Juniperus

virginiana)

Southwestern

Several perennial grass

Reduction in summer rainfall

Munson et al.

United States $^{1}$

greatly reduce cover of perennial

(2012, 2013)

grasses as compared to woody

species

Southern New

Bouteloua eriopoda

Perennial grasses are more

Baez et al. (2013)

Mexico, USA

vulnerable to drought than shrubs,

which explain the shrub

encroachment into desert

grasslands

${ }^{1}$ Long-term vegetation data are from four sites in the Sonoran Desert of southern Arizona: Organ

Pipe Cactus National Monument (OPCNM), the Rincon District of Saguaro National Park

(SNP), the Desert Laboratory (DL), and the Santa Rita Experimental Range (SRER). 


\section{A3 Woody plants-grass interactions as affected by hydraulic lift and interannual rainfall variability}

$\underline{\text { Supplementary information }}$

Supplementary Table 4-1: A list of abbreviations used to refer to the model variables.

Variables Description

$P \quad$ Rainfall infiltration $\left(\mathrm{mm} \mathrm{d}^{-1}\right)$

$E T_{1} \quad$ Soil moisture losses from the shallow soil layer $\left(\mathrm{mm} \mathrm{d}^{-1}\right)$

$T_{2} \quad$ Soil moisture losses from the deep soil layer $\left(\mathrm{mm} \mathrm{d}^{-1}\right)$

$D_{1} \quad$ Drainage from the shallow soil layer $\left(\mathrm{mm} \mathrm{d}^{-1}\right)$

$D_{2} \quad$ Drainage from the deep soil layer $\left(\mathrm{mm} \mathrm{d}^{-1}\right)$

$H R \quad$ Hydraulic redistribution $\left(\mathrm{mm} \mathrm{d}^{-1}\right)$

$S_{1} \quad$ Soil moisture in the shallow soil layer

$S_{2} \quad$ Soil moisture in the deep soil layer

$\Psi_{s 1} \quad$ Soil water potential in the shallow soil layer $(\mathrm{MPa})$

$\Psi_{s 2} \quad$ Soil water potential in the deep soil layer (MPa)

$f_{c} \quad$ Fractional canopy cover of woody plants

$f_{r} \quad$ Fractional root cover of woody plants

$i_{w} \quad$ Number of woody plant individuals per unit area

$W_{l} \quad$ Biomass of woody plants at the landscape scale $\left(\mathrm{kg} \mathrm{m}^{-2}\right)$ 


\begin{tabular}{cl}
\hline$G_{l}$ & Grass biomass at the landscape scale $\left(\mathrm{kg} \mathrm{m}^{-2}\right)$ \\
$\gamma$ & Fraction of woody plants biomass relying on the shallow soil layer \\
$V_{1}$ & Vegetation carrying capacity contributed by the shallow soil layer $(\mathrm{kg}$ \\
& $\left.\mathrm{m}^{-2}\right)$ \\
$V_{2}$ & $\quad$ Vegetation carrying capacity contributed by the deep soil layer $\left(\mathrm{kg} \mathrm{m}^{-}\right.$ \\
& $\left.{ }^{2}\right)$ \\
$\overline{\mathrm{LAI}}_{w}$ & $\quad$ Average leaf area index of woody land patches $\left(\mathrm{m}^{2} \mathrm{~m}^{-2}\right)$ \\
$L A I_{l w}$ & Woody plant leaf area index at the landscape scale $\left(\mathrm{m}^{2} \mathrm{~m}^{-2}\right)$ \\
$\eta$ & Fire frequency
\end{tabular}


Supplementary Table 4-2: Parameters, parameter values, and reference sources used in the study.

\begin{tabular}{llll}
\hline Parameter Symbol Value & Refence
\end{tabular}

\begin{tabular}{llll}
\hline Total potential evapotranspiration & $P E T$ & $5 \mathrm{~mm} \mathrm{~d}^{-1}$ & Yu \& D’Odorico (2014 a) \\
Extinction coefficient of & $e_{S}$ & 0.35 & Brutsaert (1982)
\end{tabular}

shortwave radiation

Depth of the shallow soil layer $\quad Z_{1} \quad 0.25 \mathrm{~m} \quad$ Nippert et al (2012),

Depth of the deep soil layer ${ }^{1} \quad Z_{2} \quad 0.45 \mathrm{~m}$ or $0.75 \mathrm{~m} \quad$ Yu \& D'Odorico (2014 a)

and

Bhattachan et al (2012)

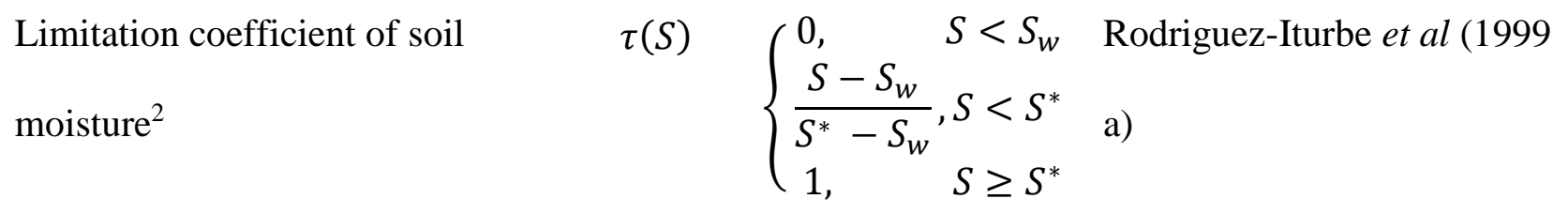

Factor reducing root hydraulic

$R_{e} \quad \frac{1}{1+\frac{\max \left(\Psi_{S 2}, \Psi_{S 1}\right)^{b}}{\Psi_{50}}}$ Ryel et al (2002)

conductance $^{3}$

$C_{r \max } \quad 0.75 \times \overline{\mathrm{LAI}}_{w} \mathrm{~mm} \quad$ Yu \& D’Odorico (2014 a)

Maximum root hydraulic

Crmax

$\mathrm{MPa}^{-1} \mathrm{~h}^{-1}$

conductance

Canopy radius of an individual of

$u \quad 1.7 \mathrm{~m}$

Caylor et al (2006)

woody plant canopy 


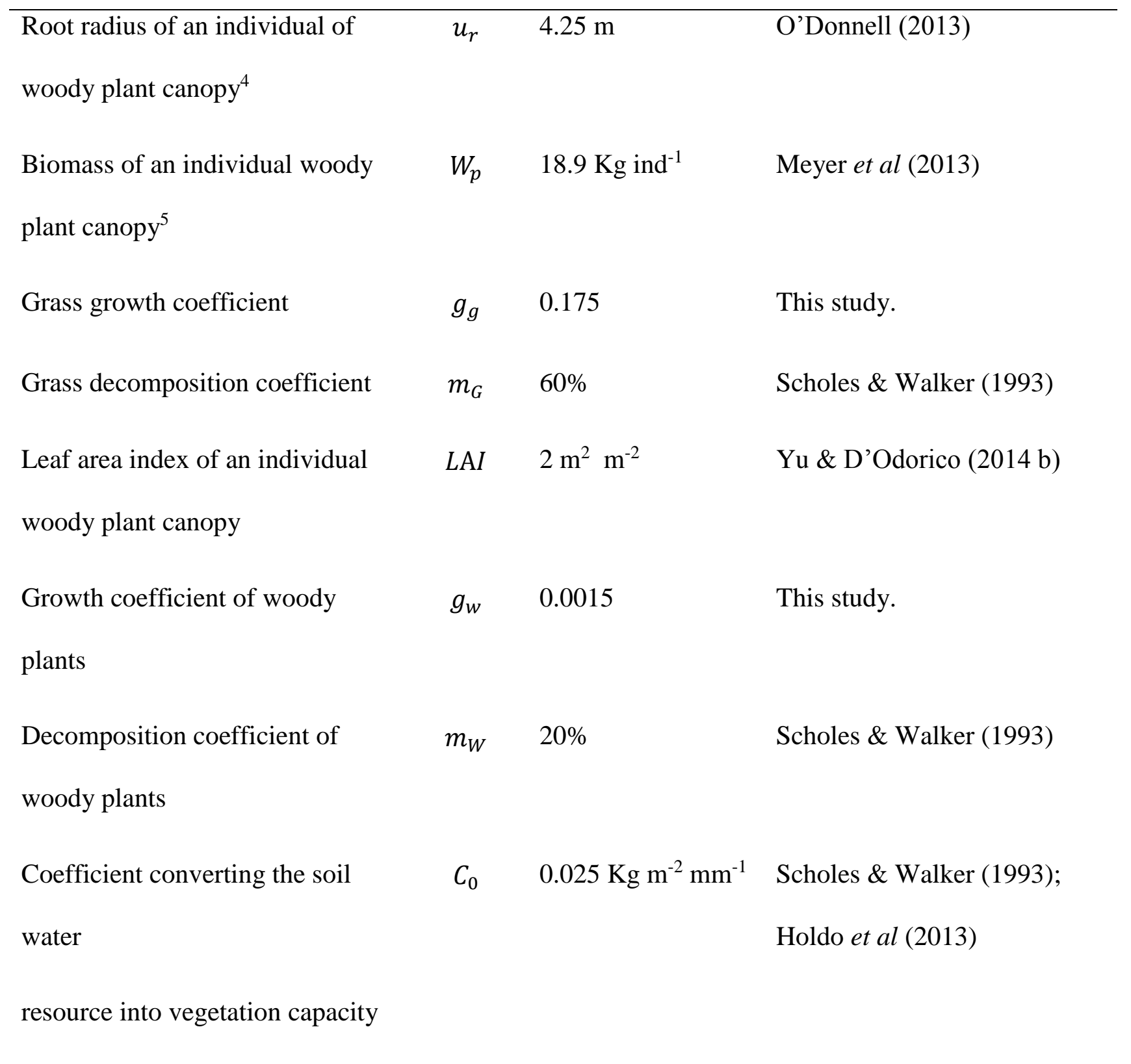

Note: ${ }^{1}$ Depth of the deep soil layer is equal to the root depth of woody plants present in the deep soil layer and thus total root depth of woody plants is 0.7 or $1 \mathrm{~m}$.

${ }^{2}$ Trees and grasses are assumed to have the same values of $S^{*}$ and $S_{w}$. Values of $S^{*}$ and $S_{w}$ can be found in Table 3 .

${ }^{3} \Psi_{50}$ is the soil water potential where soil-root conductance is reduced by $50 \%$ and $b$ an empirical constant. $\Psi_{50}=-1 \mathrm{MPa}$ and $b=3.22$ (Ryel et al., 2002). 
${ }^{4} u_{r}$ is determined based on the field data from O'Donnell et al (2013) who found that the root radius is generally 2-3 times the canopy radius of woody plants.

${ }^{5} W_{p}$ is determined based on the field data from Meyer et al (2013) who found that woody plant biomass at landscape scale is $W_{l}=2.5 \mathrm{~kg} \mathrm{~m}^{-2}$ at $f_{c}=70 \%$.

Supplementary Table 4-3: Parameters describing various soil characteristics used in this study.

\begin{tabular}{lrccccccc}
\hline Soil types & $\Psi_{S}(\mathrm{MPa})$ & $\mathrm{d}$ & $K_{S}\left(\mathrm{~mm} \mathrm{~h}^{-1}\right)$ & $\mathrm{n}$ & $\beta$ & $S_{w}$ & $S^{*}$ & $S_{f c}$ \\
& & & & & & & & \\
\hline Sand & $-1.85 \times 10^{-3}$ & 4.05 & 100 & 0.35 & 12.1 & 0.11 & 0.33 & 0.35 \\
& & & & & & & & \\
Loamy sand & $-0.88 \times 10^{-3}$ & 4.38 & 50 & 0.42 & 12.7 & 0.11 & 0.31 & 0.52
\end{tabular}

The values of these parameters are from Laio and others (2001). Following Laio and others (2001), $\beta$ is calculated as $\beta=2 \times d+4 . \Psi_{S}$ : soil parameter used to calculate soil water potential; d: soil parameter used to calculate soil water potential; $K_{s}$ : soil saturated hydraulic conductivity; n: soil porosity; $\beta$ : coefficient used to calculate drainage; $S_{w}$ : vegetation-specific wilting point at which point transpiration ceases; $S^{*}$ : vegetation-specific value of relative soil moisture above which transpiration is not limited by soil water availability; $f c$ : field capacity. 


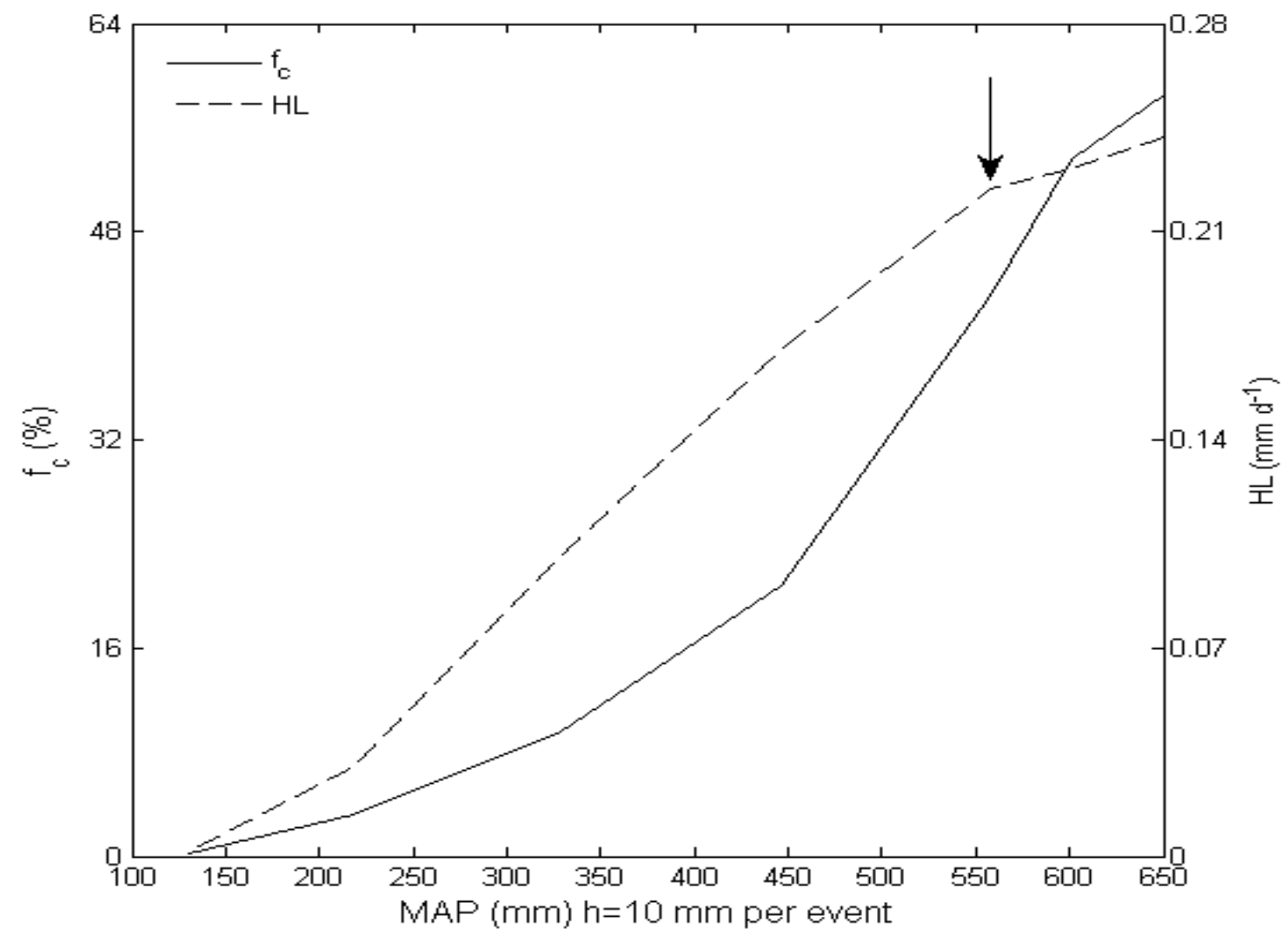

Supplementary Figure 4-1: Dependence of woody plant fractional cover (fc) and landscape scale hydraulic lift (HL) on mean annual precipitation (MAP) at steady states. The following parameters were used: mean rainfall depth, $\mathrm{h}=10 \mathrm{~mm}$ per event; root density of woody plants in the deep soil layer, $\alpha 2=0.15$. The arrow $(\downarrow)$ indicates the MAP at which the fractional root cover of woody plants (at the landscape scale) is, $\mathrm{f}_{\mathrm{r}}>0.95$. 

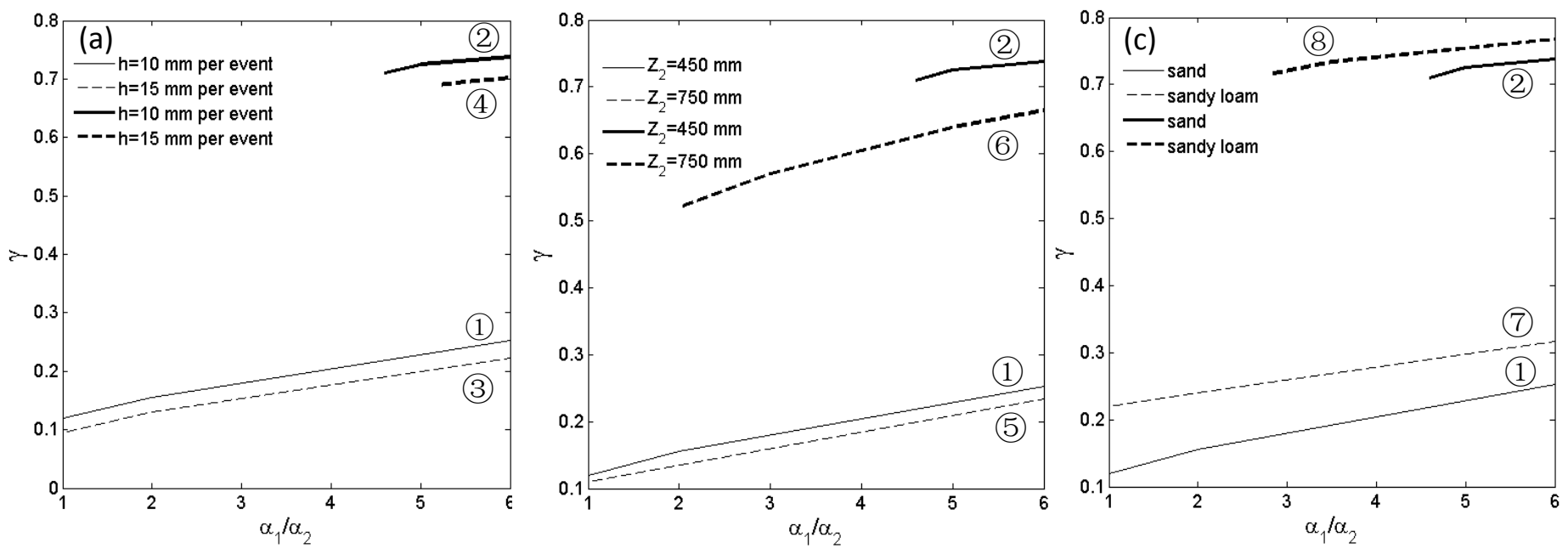

Supplementary Figure 4-2: The effects of the ratio $\left(a_{1} / a_{2}\right)$ of woody root density in the shallow and deep soil layer on the fraction $(\gamma)$ of woody plants biomass relying on the shallow soil layer. The lines of (1), (2), (3), (4), (5), (6), (7), and (8) correspond to the the lines (1), (2), (3), (4), (5), 6), (7), and (8) in Figure 4-2 (i.e., in the presence of hydraulic lift (HL)). 

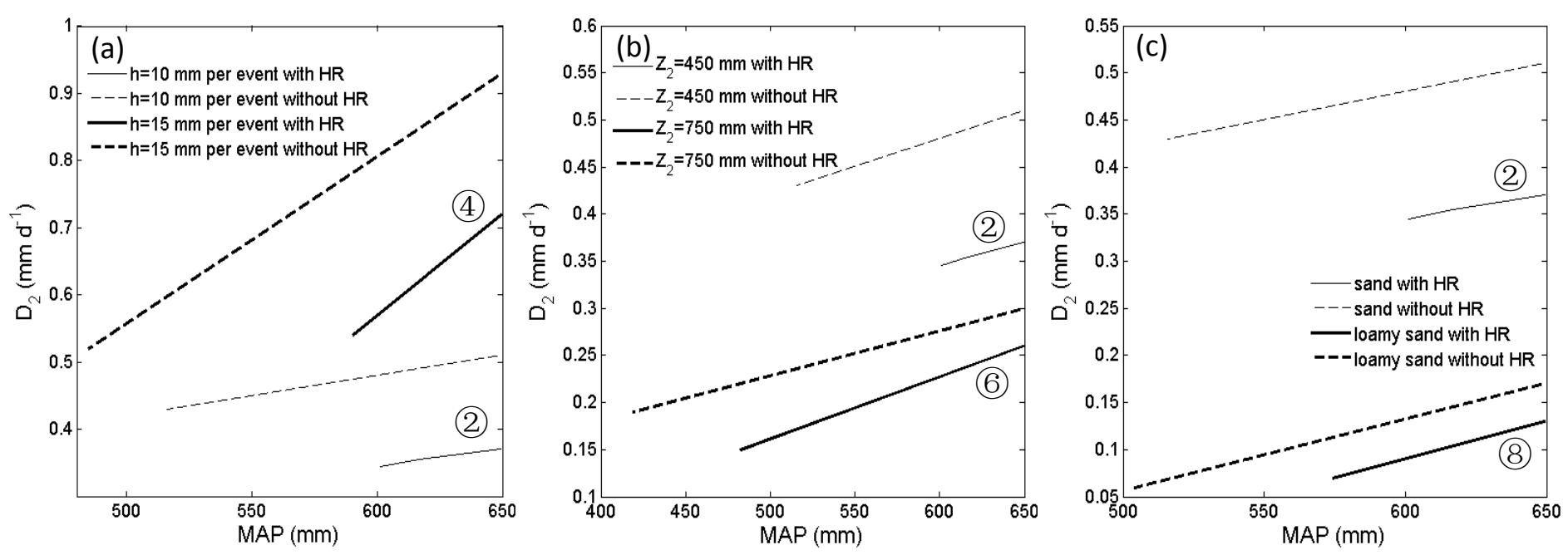

Supplementary Figure 4-3: The effects rainfall frequency $(\lambda)$ and mean annual precipitation (MAP) on drainage $\left(\mathrm{D}_{2}\right)$ from the deep soil layer (i.e., from beneath the root zone of woody plants). The lines of (2), (4), (6) and (8) correspond to the lines of (2), (4), (6) and (8) in Figure 4-2 (i.e., in the presence of hydraulic lift (HL)). 
A4 Plants with Crassulacean Acid Metabolism outcompete grasses under carbon dioxide enrichment and drought

\section{$\underline{\text { Supplementary Information }}$}

Supplementary Table 5-1: Results (P values) of five-way factorial ANOVA on total biomass, root/shoot biomass ratio, relative aboveground plant water content (RAWC), and aboveground plant carbon isotope discrimination $(\Delta)$.

\begin{tabular}{|c|c|c|c|c|c|}
\hline & $\mathrm{df}$ & TB & BA & RAWC & $\Delta$ \\
\hline $\mathrm{CO}_{2}$ & 1 & 0.0232 & 0.0012 & - & $<.0001$ \\
\hline Water & 1 & $<.0001$ & $<.0001$ & $<.0001$ & $<.0001$ \\
\hline Species & 1 & $<.0264$ & $<.0001$ & $<.0001$ & $<.0001$ \\
\hline Competition & 1 & - & 0.0036 & $<.0001$ & - \\
\hline Time & 1 & $<.0001$ & $<.0001$ & 0.0002 & 0.0001 \\
\hline $\mathrm{CO}_{2} \times$ water & 1 & $<.0097$ & - & - & $<.0001$ \\
\hline $\mathrm{CO}_{2} \times$ species & 1 & $<.0001$ & - & 0.0122 & $<.0001$ \\
\hline $\mathrm{CO}_{2} \times$ competition & 1 & 0.0468 & - & 0.0225 & 0.0004 \\
\hline $\mathrm{CO}_{2} \times$ time & 1 & - & - & 0.0041 & $<.0001$ \\
\hline Water $\times$ species & 1 & $<.0001$ & $<.0001$ & $<.0001$ & $<.0001$ \\
\hline Waterxcompetition & 1 & $<.0001$ & 0.0273 & 0.001 & - \\
\hline Waterxtime & 1 & $<.0001$ & - & - & 0.0014 \\
\hline Species $\times$ competition & 1 & $<.0001$ & - & $<.0001$ & - \\
\hline Species $\times$ time & 1 & - & $<.0001$ & - & $<.0001$ \\
\hline Competition $\times$ time & 1 & - & - & 0.0145 & 0.044 \\
\hline $\mathrm{CO}_{2} \times$ water $\times$ species & 1 & - & - & - & - \\
\hline $\mathrm{CO}_{2} \times$ water $\times$ competition & 1 & $<.0001$ & - & - & - \\
\hline $\mathrm{CO}_{2} \times$ water $\times$ time & 1 & - & - & - & $<.0001$ \\
\hline $\mathrm{CO}_{2} \times$ species $\times$ competition & 1 & $<.0001$ & - & - & - \\
\hline $\mathrm{CO}_{2} \times$ species $\times$ time & 1 & - & - & - & $<.0001$ \\
\hline $\mathrm{CO} 2 \times$ competition $\times$ time & 1 & - & - & - & - \\
\hline Water $\times$ speciexcompetion & 1 & $<.0001$ & 0.0412 & $<.0001$ & - \\
\hline Water $\times$ species $\times$ time & 1 & $<.0001$ & - & - & $<.0001$ \\
\hline Water $\times$ competition $\times$ time & 1 & - & - & - & - \\
\hline Species $\times$ competition $\times$ time & 1 & - & - & - & - \\
\hline
\end{tabular}




\begin{tabular}{|c|c|c|c|c|c|}
\hline $\mathrm{CO}_{2} \times$ wate $\times$ species $\times$ competition & 1 & 0.0015 & - & - & - \\
\hline $\mathrm{CO}_{2} \times$ water $\times$ species $\times$ time & 1 & - & - & - & $<.0001$ \\
\hline $\mathrm{CO}_{2} \times$ water $\times$ competition $\times$ time & 1 & - & 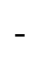 & - & 0.0463 \\
\hline $\mathrm{CO}_{2} \times$ species $\times$ competition $\times$ time & 1 & - & - & 0.0084 & - \\
\hline Water $\times$ species $\times$ competition $\times$ time & 1 & - & - & - & - \\
\hline $\mathrm{CO}_{2} \times$ water $\times$ species $\times$ competition $\times$ time & 1 & - & - & - & - \\
\hline
\end{tabular}

Note: “-” means no significance $(\mathrm{P}>0.05)$. 
Supplementary Table 5-2: Observations of vegetation growth in CAM alone (C), grass alone (G), mixture $(\mathrm{CM}, \mathrm{GM})$ in each treatment.

\begin{tabular}{|c|c|c|c|}
\hline Treatment & $\begin{array}{l}\text { Vegetation } \\
\text { types }\end{array}$ & Vegetation traits in September 25th & $\begin{array}{l}\text { Vegetation traits in } \\
\text { October 16th }\end{array}$ \\
\hline $\begin{array}{l}\text { High } \mathrm{CO}_{2} \text { and well- } \\
\text { watered conditions }\end{array}$ & $\mathrm{C}$ & $\begin{array}{l}\text { Dark green, many } 0.5-1 \mathrm{~cm} \text { "leaves", } \\
\text { larger leaf-type structures at base }\end{array}$ & Dark green, areoles $0-2 \mathrm{~cm}$ \\
\hline \multirow[t]{3}{*}{ (HCHW) } & G & Dark green, straight & $\begin{array}{l}\text { Green and straight, brown } \\
\text { and curly at tips }\end{array}$ \\
\hline & $\mathrm{CM}$ & $\begin{array}{l}\text { Darkgreen, many } 0.5-1 \mathrm{~cm} \text { "leaves", } \\
\text { larger leaf-type structures at base }\end{array}$ & $\begin{array}{l}\text { Green, some wrinkles, and } \\
\text { some areoles } 0-1 \mathrm{~cm}\end{array}$ \\
\hline & GM & Dark green, straight & Green, straight \\
\hline $\begin{array}{l}\mathrm{High} \mathrm{CO}_{2} \text { and droght } \\
\text { conditions }\end{array}$ & $\mathrm{C}$ & $\begin{array}{l}\text { Green, wrinkly, some "leaves" } \sim 0.5- \\
1 \mathrm{~cm}\end{array}$ & $\begin{array}{l}\text { Green, wrinkly, brown at } \\
\text { base }\end{array}$ \\
\hline \multirow[t]{3}{*}{ (HCLW) } & $\mathrm{G}$ & $\begin{array}{l}\text { Mostly green, some brown at tips, } \\
\text { curly }\end{array}$ & Brown, appear to be dead \\
\hline & $\mathrm{CM}$ & Dark green, some "leaves" $\sim 0.5-1 \mathrm{~cm}$ & $\begin{array}{l}\text { Green, wrinkly, brown at } \\
\text { base }\end{array}$ \\
\hline & GM & $\begin{array}{l}\text { Mostly green, some brown at tips, } \\
\text { curly }\end{array}$ & Brown, appear to be dead \\
\hline
\end{tabular}




\begin{tabular}{|c|c|c|c|}
\hline Low $\mathrm{CO}_{2}$ and well- & $\mathrm{C}$ & Many "leaves" $\sim 0.5-1 \mathrm{~cm}$, dark green, & Green, areoles $0-1.5 \mathrm{~cm}$ \\
\hline watered conditions & & some larger leaf-like structures at base & \\
\hline \multirow{6}{*}{ (LCHW) } & G & Mostly green, mostly straight & Half green and straight, \\
\hline & & & half brown and curly \\
\hline & $\mathrm{CM}$ & Green, wrinkly, some "leaves" 0- & Green, areoles $0-1.5 \mathrm{~cm}$ \\
\hline & & $0.5 \mathrm{~cm}$ & \\
\hline & GM & Half brown, half green, mostly curly, & Half green and straight, \\
\hline & & some straight & half brown \\
\hline Low $\mathrm{CO}_{2}$ and droght & $\mathrm{C}$ & Dark green, wrinkly, no "leaves" & Green, wrinkly, no areoles \\
\hline conditions & G & Half brown, half green, curly & Very brown, appear to be \\
\hline \multirow[t]{3}{*}{$(\mathrm{LCLW})$} & & & dead \\
\hline & $\mathrm{CM}$ & Dark green, wrinkly, no "leaves" & Green, wrinkly, no areoles \\
\hline & GM & Mostly brown, curly & $\begin{array}{l}\text { Very brown, appear to be } \\
\text { dead }\end{array}$ \\
\hline
\end{tabular}




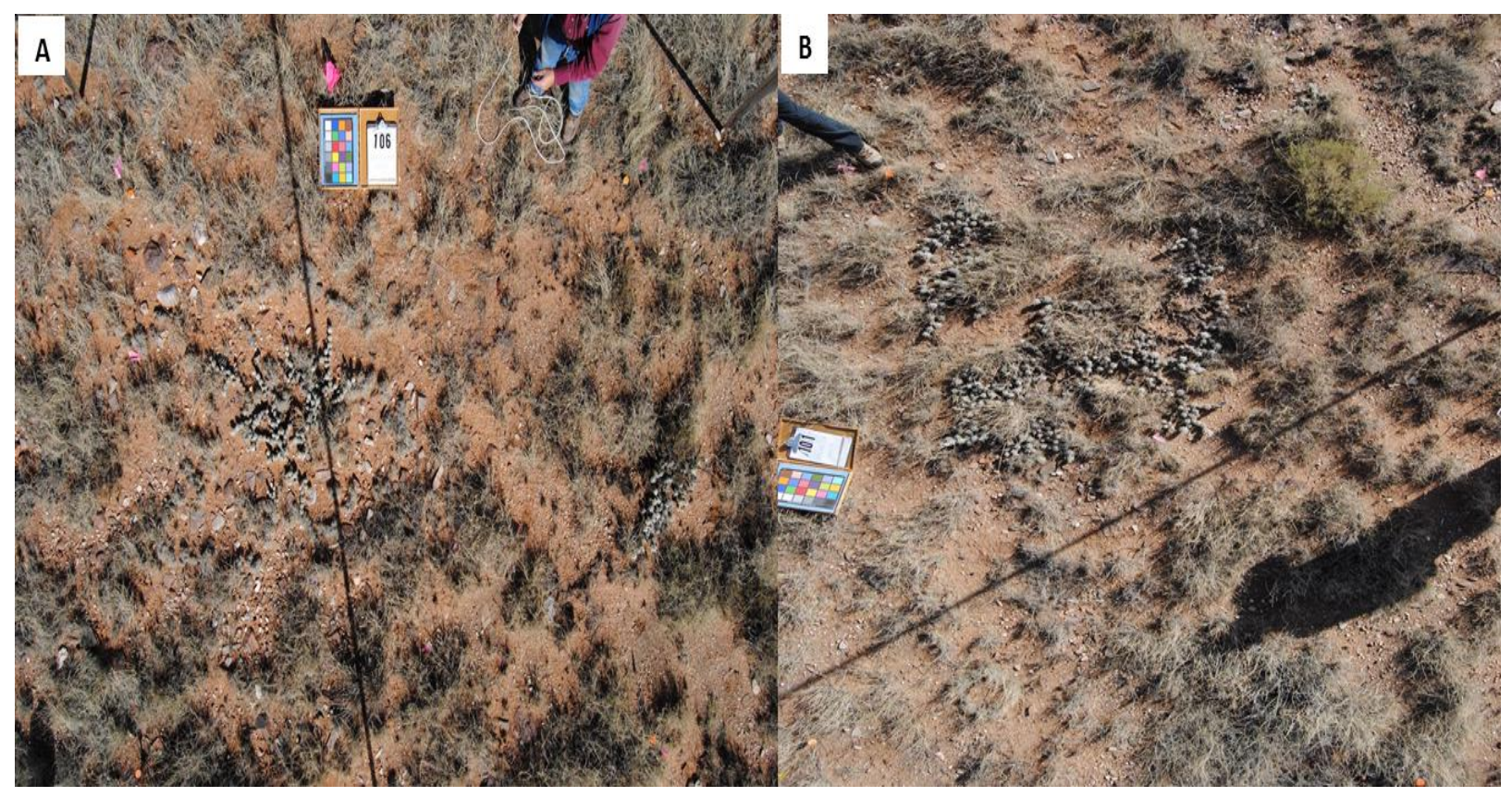

Supplementary Figure 5-1: Observations of C. imbricata (CAM) and B. eriopoda $\left(\mathrm{C}_{4}\right.$ grass) in Sevilleta National Wildlife Refuge in 1993 (A) and 2015 (B). 


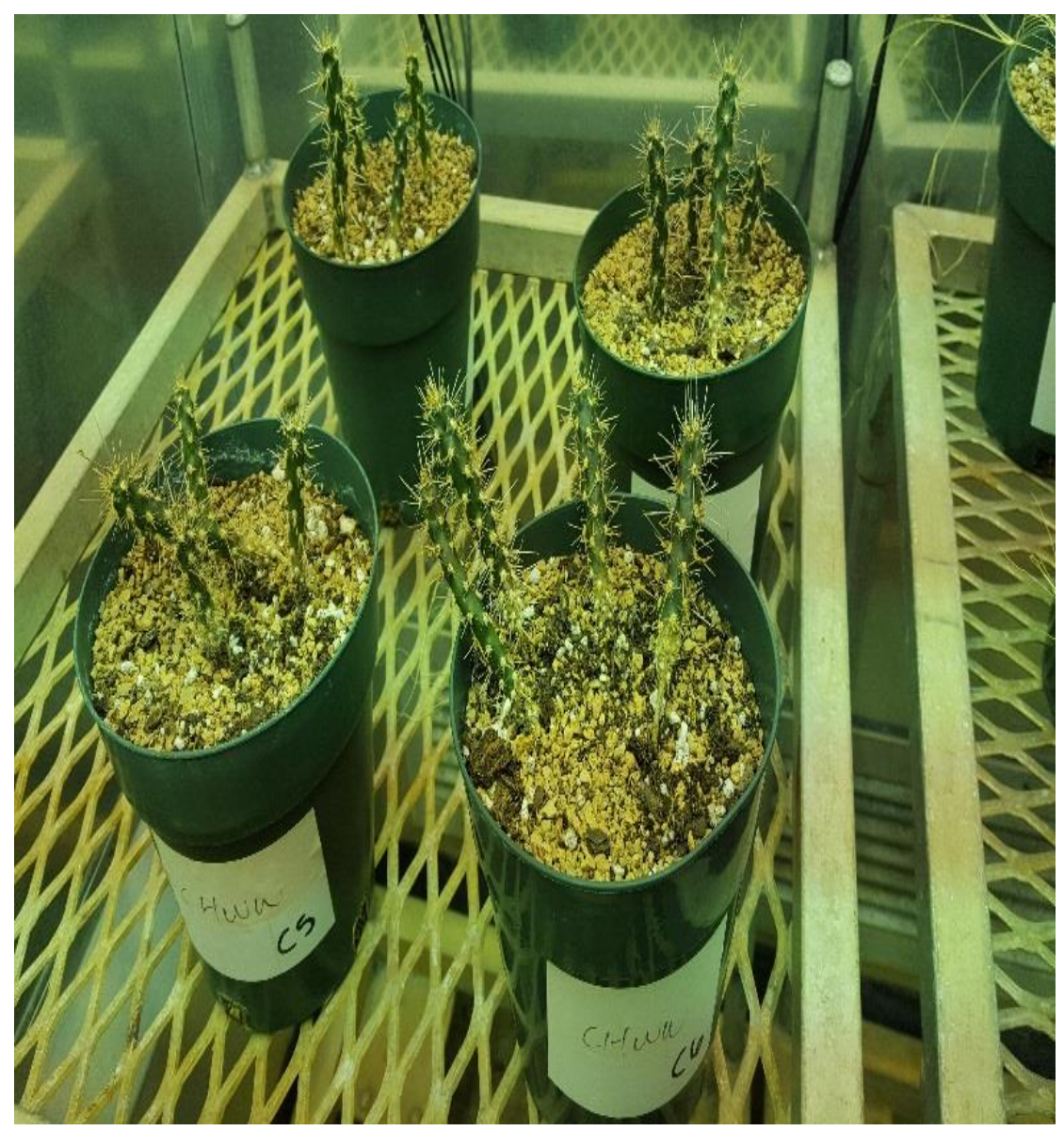

Supplementary Figure 5-2: Observations of $C$. imbricata which are stems with cylindrical shape. 

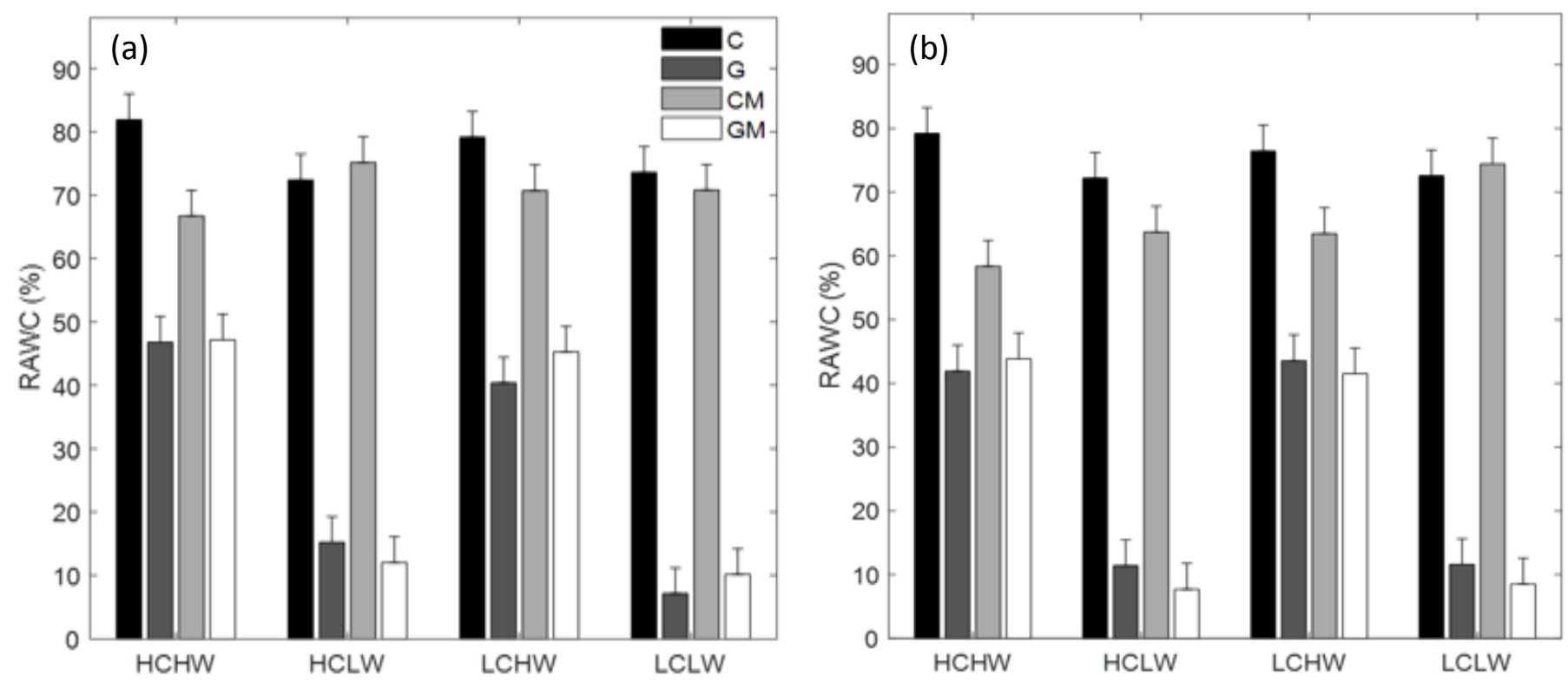

Supplementary Figure 5-3: Relative abovegorund plant water content (RAWC, \%) of $C$.

imbricata $(\mathrm{CAM})$ and B. eriopoda $\left(\mathrm{C}_{4}\right.$ grass $)$ in $\mathrm{CAM}$ alone $(\mathrm{C})$, grass alone $(\mathrm{G})$, mixture $(\mathrm{CM}$, GM) in the first stage (a, November 13th 2015) and the second stage (b, December 23th 2015) in each treamtment. $\mathrm{HCHW}$ refers to high $\mathrm{CO} 2$ and well-watered conditions; HCLW refers to high $\mathrm{CO}_{2}$ and drought conditions; LCHW refers to low $\mathrm{CO}_{2}$ and well-watered conditions; LCLW refers to low $\mathrm{CO}_{2}$ and drought conditions. The error bars represent $95 \%$ confidence intervals. 


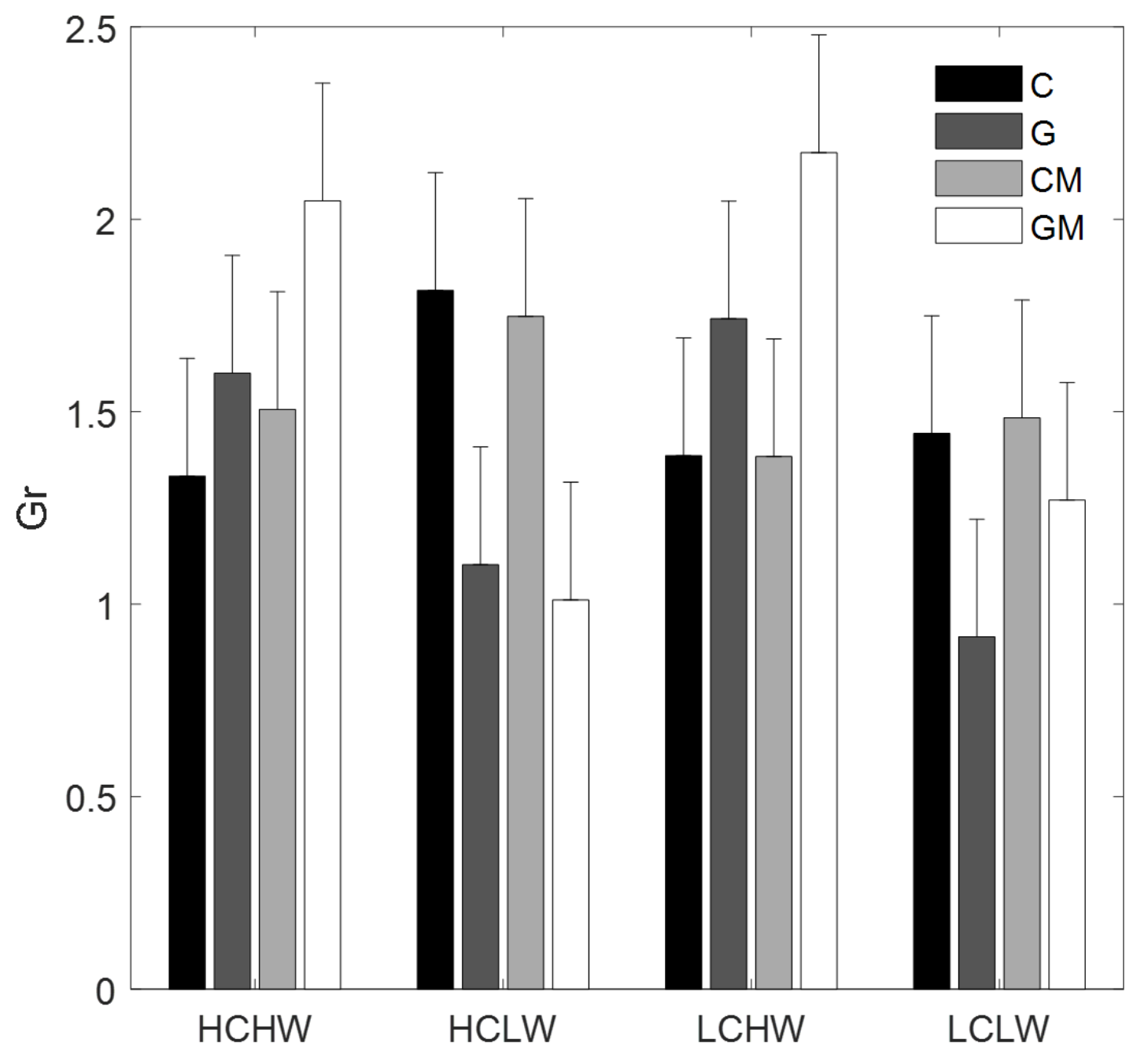

Supplementary Figure 5-4: Supplementary Figure 5-4: Growth rate (the total biomass ratio between the first and the second stage) of C. imbricata (CAM) and B. eriopoda ( $\mathrm{C}_{4}$ grass) in CAM alone $(\mathrm{C})$, grass alone $(\mathrm{G})$, mixture $(\mathrm{CM}, \mathrm{GM})$ in each treatment. Symbols for each treatment are the same as Supplementary Figure 5.3. The error bars represent 95\% confidence intervals. 

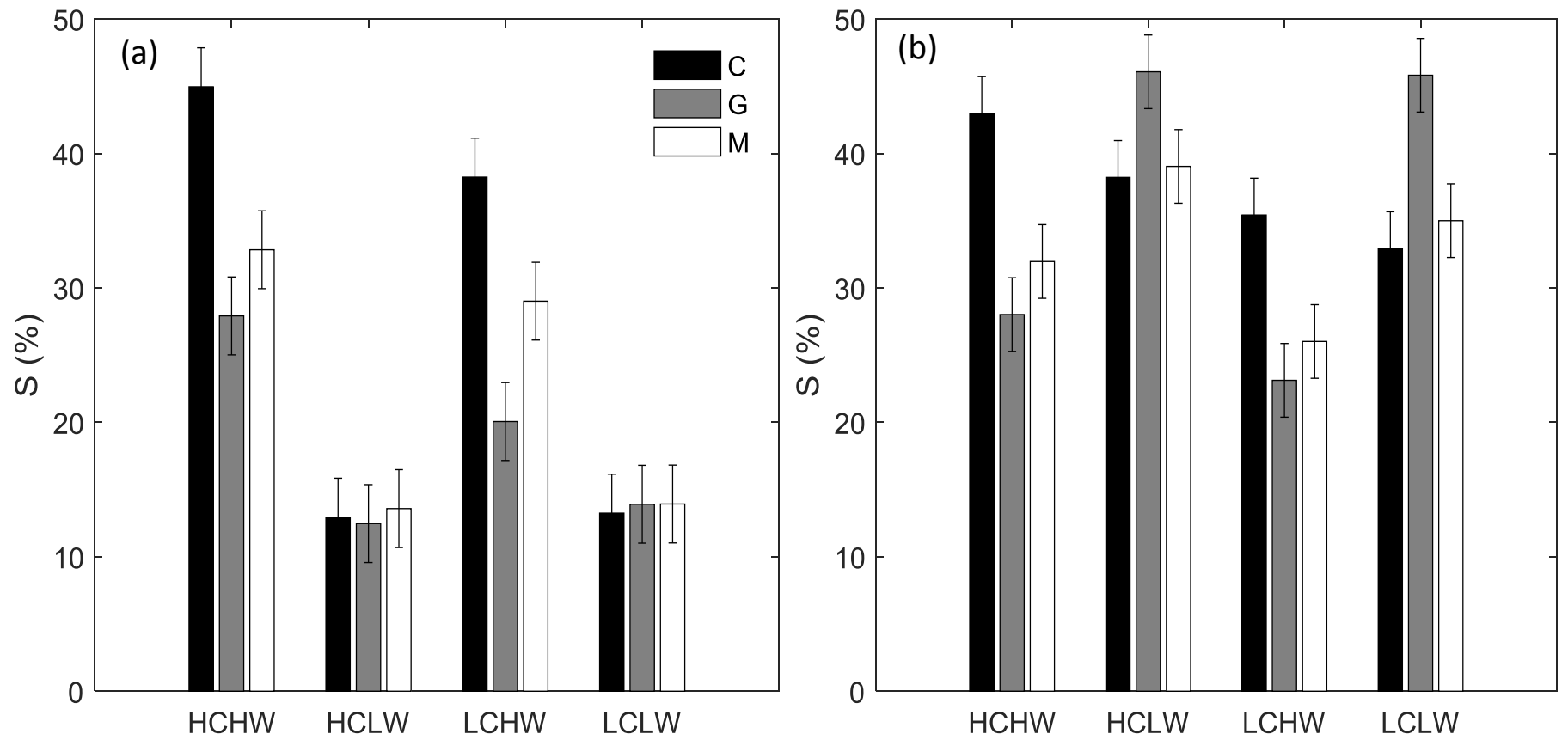

Supplementary Figure 5-5: Soil moisture content (S, \%) in CAM alone (C), grass alone (G), mixture (M) in the first stage (a, November 13th 2015) and the second stage (b, December 23th 2015) in each treatment. Symbols for each treatment are the same as Supplementary Figure 5.3. The error bars represent $95 \%$ confidence intervals. 

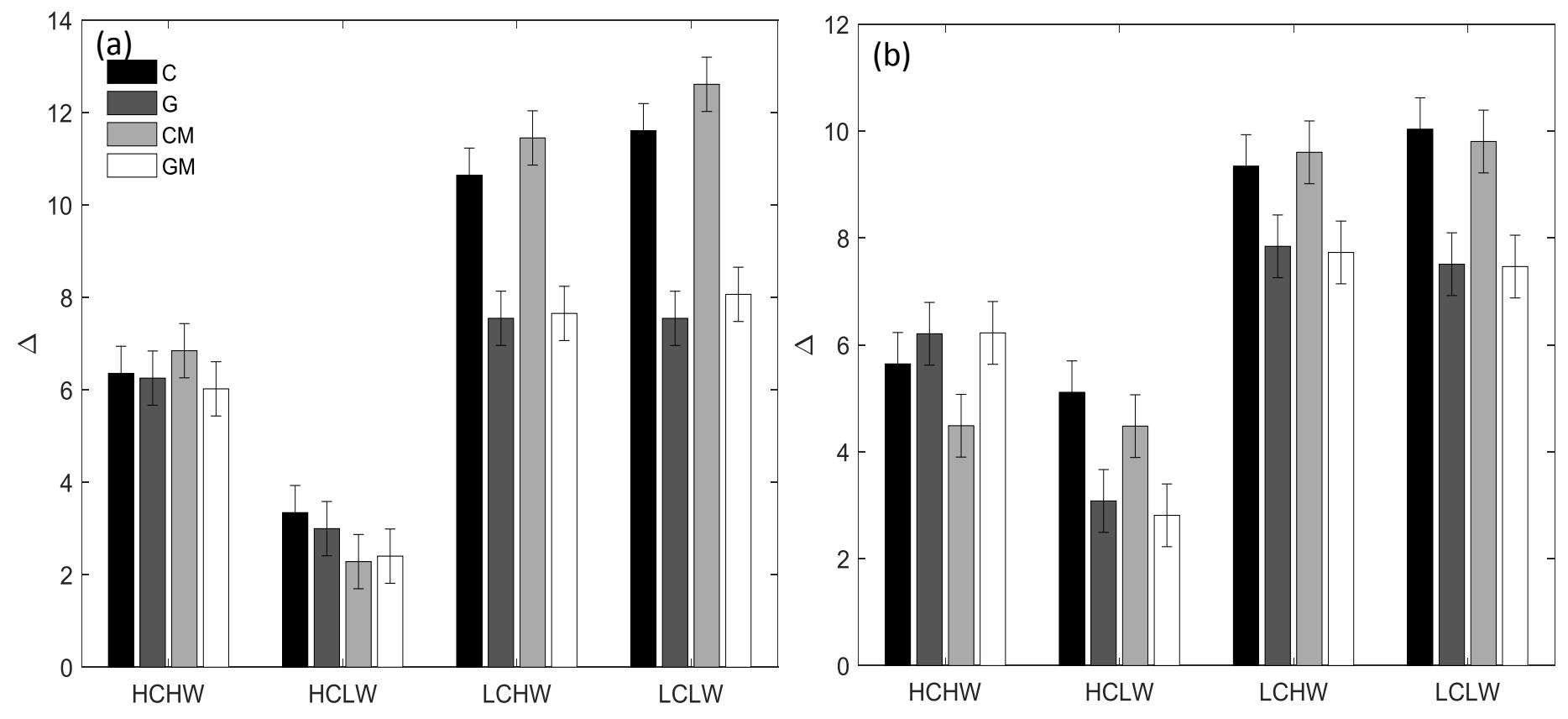

Supplementary Figure 5-6: Aboveground plant carbon isotope discrimination $(\Delta)$ of $C$. imbricata (CAM) and B. eriopoda $\left(\mathrm{C}_{4}\right.$ grass) in $\mathrm{CAM}$ alone $(\mathrm{C})$, grass alone $(\mathrm{G})$, mixture $(\mathrm{CM}, \mathrm{GM})$ in the first stage (a, Novenber 13th 2015) and the second stage (b, December 23th 2015) in each treamtment. Symbols for each treatment are the same as Supplementary Figure 5.3. The error bars represent $95 \%$ confidence intervals. 

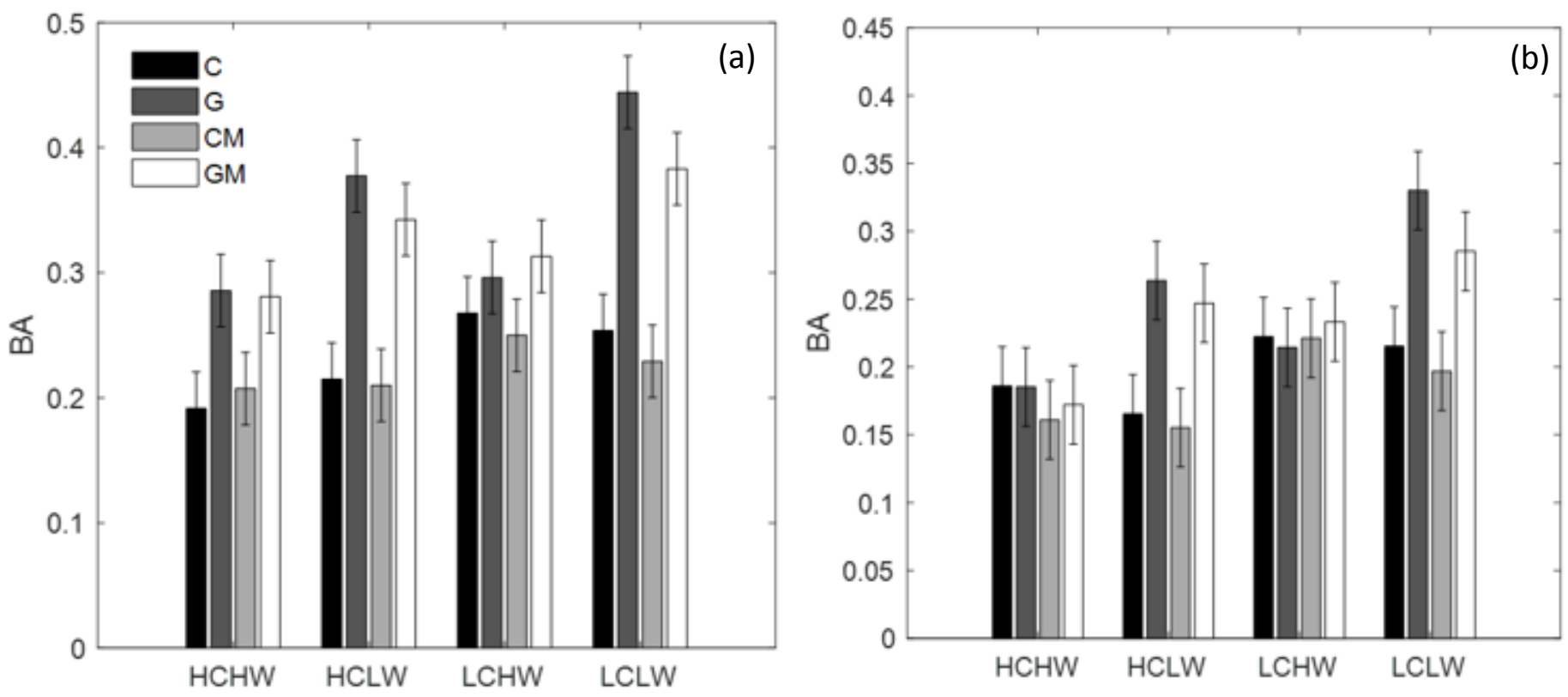

Supplementary Figure 5-7: Root/shoot ratio (BA) of C. imbricata (CAM) and B. eriopoda $\left(\mathrm{C}_{4}\right.$ grass) in CAM alone (C), grass alone $(\mathrm{G})$, mixture (CM, GM) in the first stage (a, November 13th 2015) and the second stage (b, December 23th 2015) in each treatment. Symbols for each treatment are the same as Supplementary Figure 5.3. The error bars represent $95 \%$ confidence intervals. 

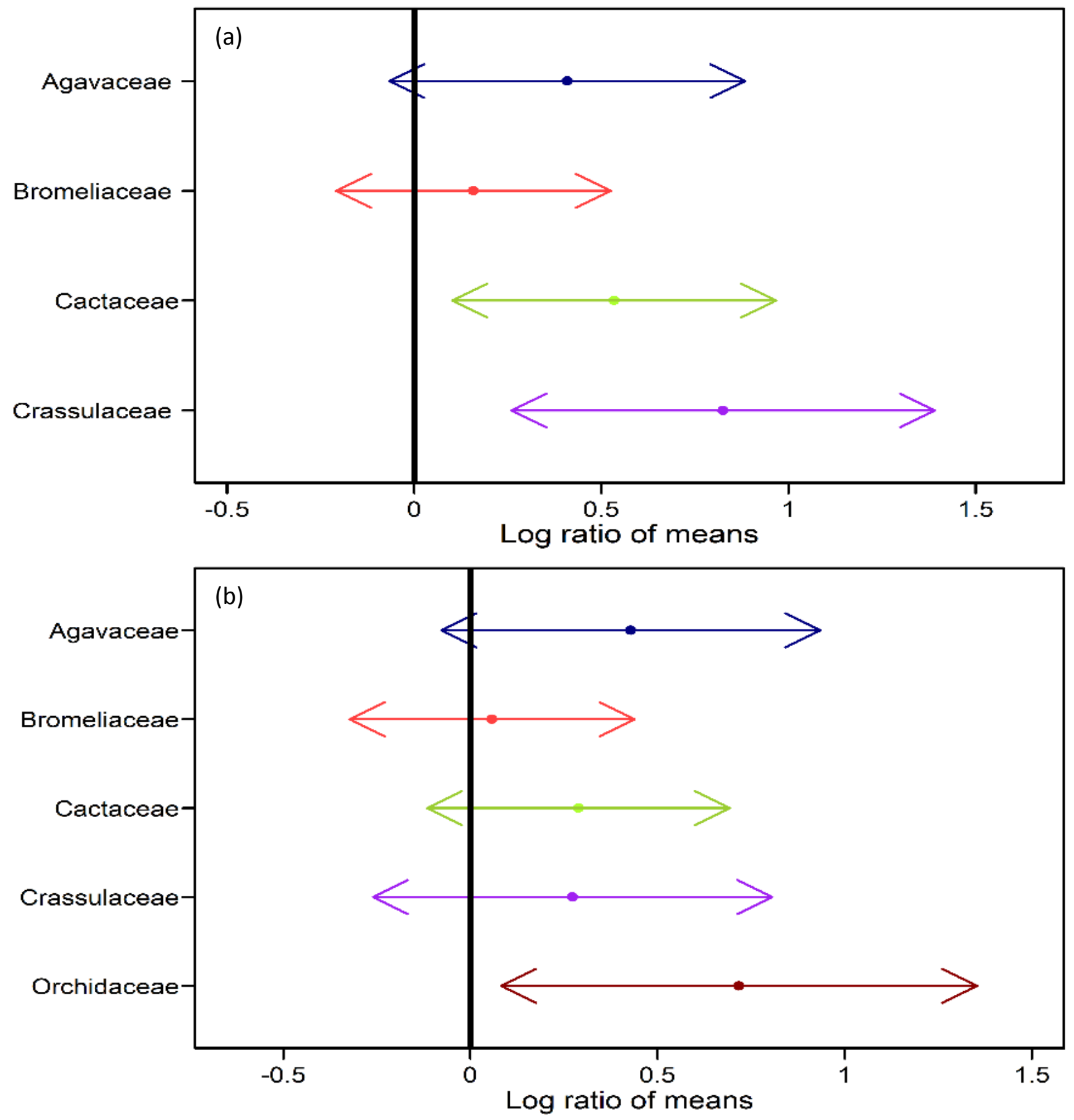

Supplementary Figure 5-8: Meta-analysis of $\log$ ratio of daily $\mathrm{CO}_{2}$ uptake (a) and biomass (b) under elevated $\mathrm{CO}_{2}$ concentrations to ambient $\mathrm{CO}_{2}$ concentrations for different families of consititude CAM plants, including 'Agavaceae', 'Bromeliaceae', 'Cactaceae', 'Crassulaceae', 'Orchidaceae'. 
Values are log ratio of means $\pm 95 \%$ confidence intervals of published results. Data averaged for daily $\mathrm{CO}_{2}$ uptake include: Agavaceae (Agave deserti ${ }^{1}$, Agave deserti ${ }^{2}$, Agave salmiana ${ }^{3}$, Agave salmiana ${ }^{4}$, Agave vilmoriniana (small plants in dry environments) ${ }^{5}$, Agave vilmoriniana (small plants in wet environments) ${ }^{5}$, Agave vilmoriniana (large plants in dry environments) ${ }^{5}$, Agave vilmoriniana (large plants in wet environments) ${ }^{5}$; Bromeliaceae (Aechmea 'Maya' ${ }^{6}$, Ananas comosus (day/night temperature: $\left.30 / 20{ }^{\circ} \mathrm{C}\right)^{7}$, Ananas comosus (day/night temperature: $\left.30 / 25^{\circ} \mathrm{C}\right)^{7}$, Ananas comosus (day/night temperature: $\left.30 / 25^{\circ} \mathrm{C}\right)^{7}$, Aechmea fasciata ${ }^{8}$, Tillandsia fasciculata $^{8}$, Tillandsia fasciculata $^{9}$, Tillandsia elongata ${ }^{9}$, Tillandsia subulifera ${ }^{9}$, Tillandsia juncea ${ }^{9}$; Cactaceae (Ferocactus acantlwdes ${ }^{1}$, Hylocereus undatus $^{10}$, Hylocereus undatus ${ }^{11}$, Selenicereus megalanthus ${ }^{11}$, Opuntia ficus-indica (basal cladodes) ${ }^{12}$, Opuntia ficus-indica (daughter cladodes) ${ }^{12}$, Opuntia ficus-indica ${ }^{13}$, Stenocereus queretaroensi ${ }^{4}$ ); Crassulaceae (Sedum alfredii (control) $)^{14}$, Sedum alfredii $\left(\mathrm{Cd} 5 ; \mathrm{Cd}\left(\mathrm{NO}_{3}\right)_{2} \cdot 4 \mathrm{H}_{2} \mathrm{O} \text { solution: } 5 \mathrm{mg} \mathrm{kg}^{-1}\right)^{14}$, Sedum alfredii $(\mathrm{Cd} 50$; $\mathrm{Cd}\left(\mathrm{NO}_{3}\right)_{2} \cdot 4 \mathrm{H}_{2} \mathrm{O}$ solution: $\left.50 \mathrm{mg} \mathrm{kg}^{-1}\right)^{14}$. Data averaged for biomass include: Agavaceae (Agave deserti ${ }^{2}$, Agave deserti ${ }^{1}$, Agave vilmoriniana (small plants in dry environments) ${ }^{5}$, Agave vilmoriniana (small plants in wet environments) ${ }^{5}$, Agave vilmoriniana (large plants in dry environments) ${ }^{5}$, Agave vilmoriniana (large plants in wet environments) ${ }^{5}$ ); Bromeliaceae

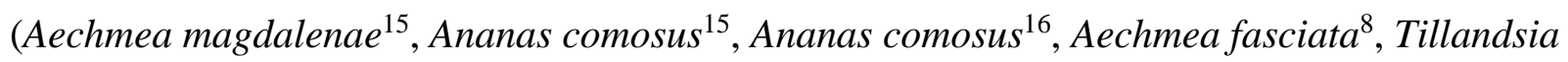
fasciculata $^{8}$, Tillandsia fasciculata $^{9}$, Tillandsia elongata ${ }^{9}$, Tillandsia subulifera ${ }^{9}$, Tillandsia juncea ${ }^{9}$ ); Cactaceae (Ferocactus acantlwdes ${ }^{1}$, Hylocereus undatus (shoot) ${ }^{11}$, Selenicereus megalanthus (shoot) ${ }^{11}$, Opuntia ficus-indica (basal cladodes) $)^{12}$, Opuntia ficus-indica (daughter cladodes) ${ }^{12}$, Opuntia ficus-indica ${ }^{17}$, Opuntia ficus-indica $\left.{ }^{18}\right)$; Crassulaceae (Kalanchoë pinnata ${ }^{19}$, Sedum alfredii (control $)^{14}$, Sedum alfredii $\left(\mathrm{Cd} 5 ; \mathrm{Cd}\left(\mathrm{NO}_{3}\right)_{2} \cdot 4 \mathrm{H}_{2} \mathrm{O} \text { solution: } 5 \text { mg kg }{ }^{-1}\right)^{14}$, Sedum 
alfredii $\left.\left(\mathrm{Cd} 50 ; \mathrm{Cd}\left(\mathrm{NO}_{3}\right)_{2} \cdot 4 \mathrm{H}_{2} \mathrm{O} \text { solution: } 50 \mathrm{mg} \mathrm{kg}^{-1}\right)^{14}\right)$; Orchidaceae (Arachnis hookeriana $\times$ Ascocenda Madame Kenny ${ }^{16}$, Arachnis hookeriana $\times$ Ascocenda Madame Kenny ${ }^{16}$, Arachnis hookeriana $\times$ Ascocenda Madame Kenny (shoot) ${ }^{17}$, Arachnis hookeriana $\times$ Ascocenda Madame Kenny (root) ${ }^{17}$. The results showed that as compared to ambient $\mathrm{CO}_{2}$ concentrations daily $\mathrm{CO}_{2}$ uptake under elevated $\mathrm{CO}_{2}$ concentrations increased by $51 \%$ (Agavaceae), $17 \%$ (Bromeliaceae), 71\% (Cactaceae), and 128\% (Crassulaceae) (Supplementary Figure 5.8a), while the increase in biomass averaged 54\% (Agavaceae), 6\% (Bromeliaceae), 34\% (Cactaceae), 31\% (Crassulaceae), 105\% (Orchidaceae) (Supplementary Figure 5.8b). 
Reference for supporting information

1. Nobel, P. S., and T. L. Hartsock. 1986. Short-term and long-term responses of crassulacean acid metabolism plants to elevated $\mathrm{CO}_{2}$. Plant Physiology 82: 604-606.

2. Graham, E. A., and P. S. Nobel. 1996. Nobel Long-term effects of a doubled atmospheric $\mathrm{CO}_{2}$ concentration on the CAM species Agave deserti. Journal of Experiment of Botany 47: $61-69$.

3. Nobel, P. S., A. A. Israel, and N. Wang. 1996. Growth, $\mathrm{CO}_{2}$ uptake, and responses of the carboxylating enzymes to inorganic carbon in two highly productive CAM species at current and doubled CO2 concentrations. Plant Cell and Environment 19: 585-592.

4. Nobel, P. S. 1996. Responses of some North American CAM plants to freezing temperatures and doubled $\mathrm{CO}_{2}$ concentrations: implications of global climate change for extending cultivation. Journal of Arid Environment 34: 187-196.

5. Idso, S. B., Kimball B. A., Anderson M. G., and S. R. Szarek. 1986. Growth response of a succulent plant, Agave vilmoriniana, to elevated $\mathrm{CO}_{2}$. Plant Physiology 80: 796-797.

6. Ceusters J., A. M. Borland, E. Londers, V. Verdoodt, C. Godts, and M. P. De Proft. 2008. Diel shifts in carboxylation pathway and metabolite dynamics in the CAM bromeliad Aechmea 'Maya' in response to elevated $\mathrm{CO}_{2}$. Annual of Botany 102: 389-397.

7. Zhu J., G. Goldstein, and D. P. Bartholomew. 1999. Gas exchange and carbon isotope composition of Ananas comosus in response to elevated $\mathrm{CO}_{2}$ and temperature. Plant Cell and Environment 22: 999-1007.

8. Monteiro J. A. F., G. Zotz, and C. Körner. 2009. Tropical epiphytes in a $\mathrm{CO}_{2}$-rich atmosphere. Acta Oecologica. 35: 60-68. 
9. Gerhard Z., W. Bogusch, P. Hietz, and N. Ketteler. 2010. Growth of epiphytic bromeliads in a changing world: The effects of $\mathrm{CO}_{2}$, water and nutrient supply. Acta Oecologica. 36: $659-665$.

10. Raveh E., M. Gersani, and P. S. Nobel. 1995. $\mathrm{CO}_{2}$ uptake and fluorescence for a shadetolerant cactus Hylocerus undatus under current and doubled $\mathrm{CO}_{2}$ concentrations. Physiologia Plantarum 93: 505-511.

11. Weissa I., Y. Mizrahia, and E. Raveh. 2010. Effect of elevated $\mathrm{CO}_{2}$ on vegetative and reproductive growth characteristics of the CAM plants Hylocereus undatus and Selenicereus megalanthus. Scientia Horticultural 123: 531-536.

12. Cui M., P. M. Miller, and P. S. Nobel. 1993. $\mathrm{CO}_{2}$ exchange and growth of the Crassulacean acid metabolism plant Opuntia ficus-indica under elevated $\mathrm{CO}_{2}$ in open-top chambers. Plant Physiology. 103: 519-524.

13. Nobel P. S., and A. A. Israel. 1994. Israel Cladode development, environmental responses of $\mathrm{CO}_{2}$ uptake, and productivity for Opuntia ficus-indica under elevated $\mathrm{CO}_{2}$. Journal of Experimental Botany 45: 295-303.

14. Li T., Q. Tao, Z. Di, F. Lu, and X. Yang. 2014. Effect of elevated $\mathrm{CO}_{2}$ concentration on photosynthetic characteristics of hyperaccumulator Sedum alfredii under cadmium stress. Journal of Integrative Plant Biology 57: 653-660.

15. Ziska L. H., K. P. Hogan, A. P. Smith, and B. G. Drake. 1991. Growth and photosynthetic response of nine tropical species with long-term exposure to doubled carbon dioxide. Oecologia 86: 383-389. 
16. Zhu J., D. P. Bartholomew, and G Goldstein. 1997. Effect of elevated carbon dioxide on the growth and physiological responses of pineapple, a species with crassulacean acid metabolism. Journal of the American Society for Horticultural Science 122: 233-237.

17. Cui M., and P. S. Nobel. 1994. Gas exchange and growth responses to elevated $\mathrm{CO}_{2}$ and light levels in the CAM species Opuntia ficus-indica. Plant Cell and Environment. 17: 935-944.

18. Nobel P.S., and A. A. Israel. 1994. Cladode development, environmental responses of $\mathrm{CO}_{2}$ uptake, and productivity for Opuntia ficusindica under elevated $\mathrm{CO}_{2}$. Journal of Experimental Botany 45: 295-303.

19. Winter K., A. Richter, B. Engelbrecht, J. Posada, A. Virgo, and M. Popp. 1997. Effect of elevated $\mathrm{CO}_{2}$ on growth and crassulacean acid metabolism activity of Kalanchoe pinnata under tropical conditions. Planta 201: 389-396.

20. Gouk S. S., J. W. H. Yong, and C. S. Hew. 1997. Effects of super-elevated $\mathrm{CO}_{2}$ on the growth and carboxylating enzymes in an epiphytic CAM orchid plantlet. Journal of Plant Physiolology 151: 129-136.

21. Li C. R., L. J. Gan, K. Xia, X. Zhou and C. S. Hew. 2002. Responses of carboxylating enzymes, sucrose metabolizing enzymes and plant hormones in a tropical epiphytic CAM orchid to CO2 enrichment. Plant Cell and Environment. 25: 369-377. 
A5 Crassulacean acid metabolism in Mesembryanthemum crystallinum: the effects of water stress, nutrient, and competition

\section{$\underline{\text { Supplementary information }}$}

Supplementary Table 6-1: Results (P values) of five-way factorial ANOVA on total biomass (TB), belowground to aboveground biomass ratio (BA), photosynthetic assimilation during the day (AD), plant leaf water potential (LWP), specific leaf area (SLA), and aboveground plant $\mathrm{N}$ $(\mathrm{APN})$

\begin{tabular}{|c|c|c|c|c|c|c|c|}
\hline & $\mathrm{df}$ & TB & $\mathrm{BA}$ & $\mathrm{A}_{\mathrm{D}}$ & LWP & SLA & APN \\
\hline Nutrient & 1 & $<.0001$ & $<.0001$ & $<.0001$ & 0.0075 & $<.0001$ & $<.0001$ \\
\hline Water & 1 & $<.0001$ & $<.0001$ & $<.0001$ & $<.0001$ & $<.0001$ & 0.0001 \\
\hline Species & 1 & $<.0001$ & $<.0001$ & $<.0001$ & $<.0001$ & - & 0.0004 \\
\hline Competition & 1 & $<.0001$ & $<.0001$ & $<.0001$ & $<.0001$ & $<.0001$ & $<.0001$ \\
\hline Time & 2 & $<.0001$ & $<.0001$ & 0.0249 & - & $<.0001$ & $<.0001$ \\
\hline Nutrient $\times$ water & 1 & $<.0001$ & $<.0001$ & $<.0001$ & $<.0001$ & $<.0001$ & 0.0023 \\
\hline Nutrient $\times$ species & 1 & - & 0.0227 & - & 0.0139 & - & 0.0002 \\
\hline Nutrient $\times$ competition & 1 & 0.0192 & - & - & 0.0098 & - & - \\
\hline Nutrient $\times$ time & 2 & - & - & 0.0211 & $<.0001$ & - & $<.0001$ \\
\hline Water $\times$ species & 1 & $<.0001$ & $<.0001$ & $<.0001$ & $<.0001$ & $<.0001$ & $<.0001$ \\
\hline Water $\times$ competition & 1 & $<.0001$ & $<.0001$ & $<.0001$ & $<.0001$ & 0.0002 & 0.0017 \\
\hline
\end{tabular}




\begin{tabular}{|c|c|c|c|c|c|c|c|}
\hline Water $\times$ time & 2 & $<.0001$ & $<.0001$ & $<.0001$ & $<.0001$ & $<.0001$ & $<.0001$ \\
\hline Species $\times$ competition & 1 & $<.0001$ & $<.0001$ & $<.0001$ & $<.0001$ & $<.0001$ & $<.0001$ \\
\hline Species $\times$ time & 2 & $<.0001$ & - & $<.0001$ & $<.0001$ & $<.0001$ & $<.0001$ \\
\hline Competition $\times$ time & 2 & $<.0001$ & $<.0001$ & $<.0001$ & $<.0001$ & 0.0008 & 0.0089 \\
\hline Nutrient $\times$ water $\times$ species & 1 & 0.0115 & 0.0213 & $<.0001$ & $<.0001$ & - & 0.0009 \\
\hline Nutrient $\times$ water $\times$ competion & 1 & - & 0.0038 & - & - & - & - \\
\hline Nutrient $\times$ water $\times$ time & 2 & - & - & 0.0012 & $<.0001$ & - & 0.033 \\
\hline Nutrient $\times$ specie $\times$ competion & 1 & 0.0007 & 0.0035 & - & - & - & - \\
\hline Nutrient $\times$ species $\times$ time & 2 & - & - & 0.0188 & $<.0001$ & 0.0046 & 0.0005 \\
\hline Nutrient $\times$ competion $\times$ time & 2 & - & - & - & - & - & - \\
\hline Water $\times$ specie $\times$ competion & 1 & $<.0001$ & $<.0001$ & $<.0001$ & $<.0001$ & $<.0001$ & 0.0402 \\
\hline Water $\times$ species $\times$ time & 2 & $<.0001$ & $<.0001$ & $<.0001$ & $<.0001$ & $<.0001$ & $<0.0001$ \\
\hline Water $\times$ competition $\times$ time & 2 & $<.0001$ & $<.0001$ & - & $<.0001$ & 0.0099 & 0.0341 \\
\hline Species $\times$ competition $\times$ time & 2 & $<.0001$ & $<.0001$ & $<.0001$ & $<.0001$ & $<.0001$ & 0.0009 \\
\hline Nutrient $\times$ water $\times$ species $\times$ competition & 1 & 0.0408 & - & - & - & - & - \\
\hline Nutrient $\times$ water $\times$ species $\times$ time & 2 & - & - & - & 0.0006 & - & - \\
\hline Nutrient $\times$ water $\times$ competition $\times$ time & 2 & - & - & - & - & - & - \\
\hline Nutrient $\times$ species $\times$ competition $\times$ time & 2 & - & - & - & - & - & 0.0224 \\
\hline Water $\times$ species $\times$ competition $\times$ time & 2 & $<.0001$ & 0.0238 & 0.0456 & $<.0001$ & 0.0112 & - \\
\hline
\end{tabular}


Nutrient $\times$ water $\times$ species $\times$ competition $\times$ time 2

Note: “-" means not significant $(\mathrm{P}>0.05)$. 


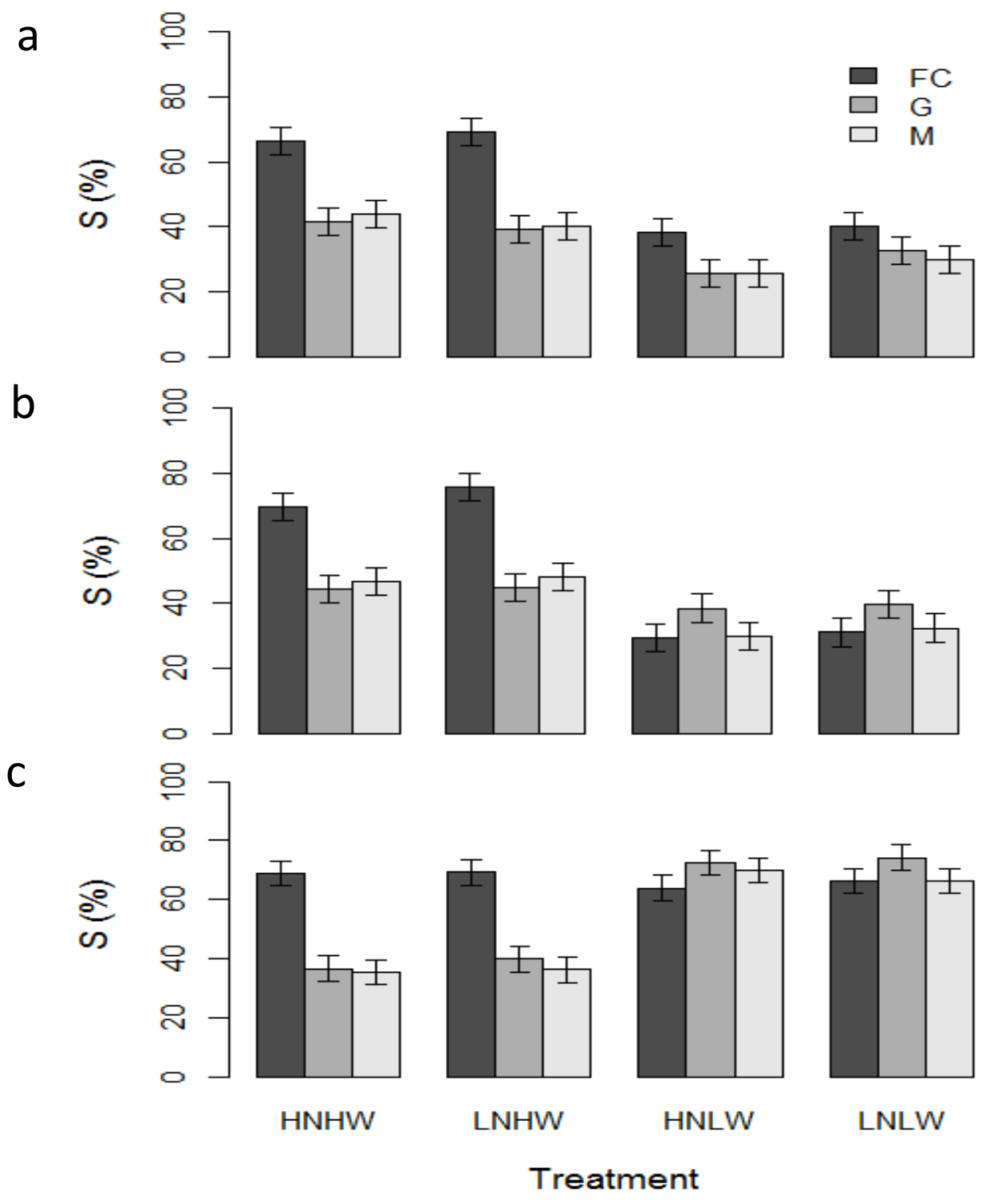

Supplementary Figure 6-1: Gravimetrically soil moisture (S) in CAM alone (FC), grass alone $(\mathrm{G})$, and mixture (M) as affected by nutrient and water conditions in the first (a), second (b), and third (c) stages. HNHW refers to high nutrient and high water conditions; LNHW refers to low nutrient and high water conditions; HNLW refers to high nutrient and low water conditions; LNLW refers to low nutrient and low water conditions. Each bar represents the mean of 6 values while error bar indicates $95 \%$ confidence intervals. 


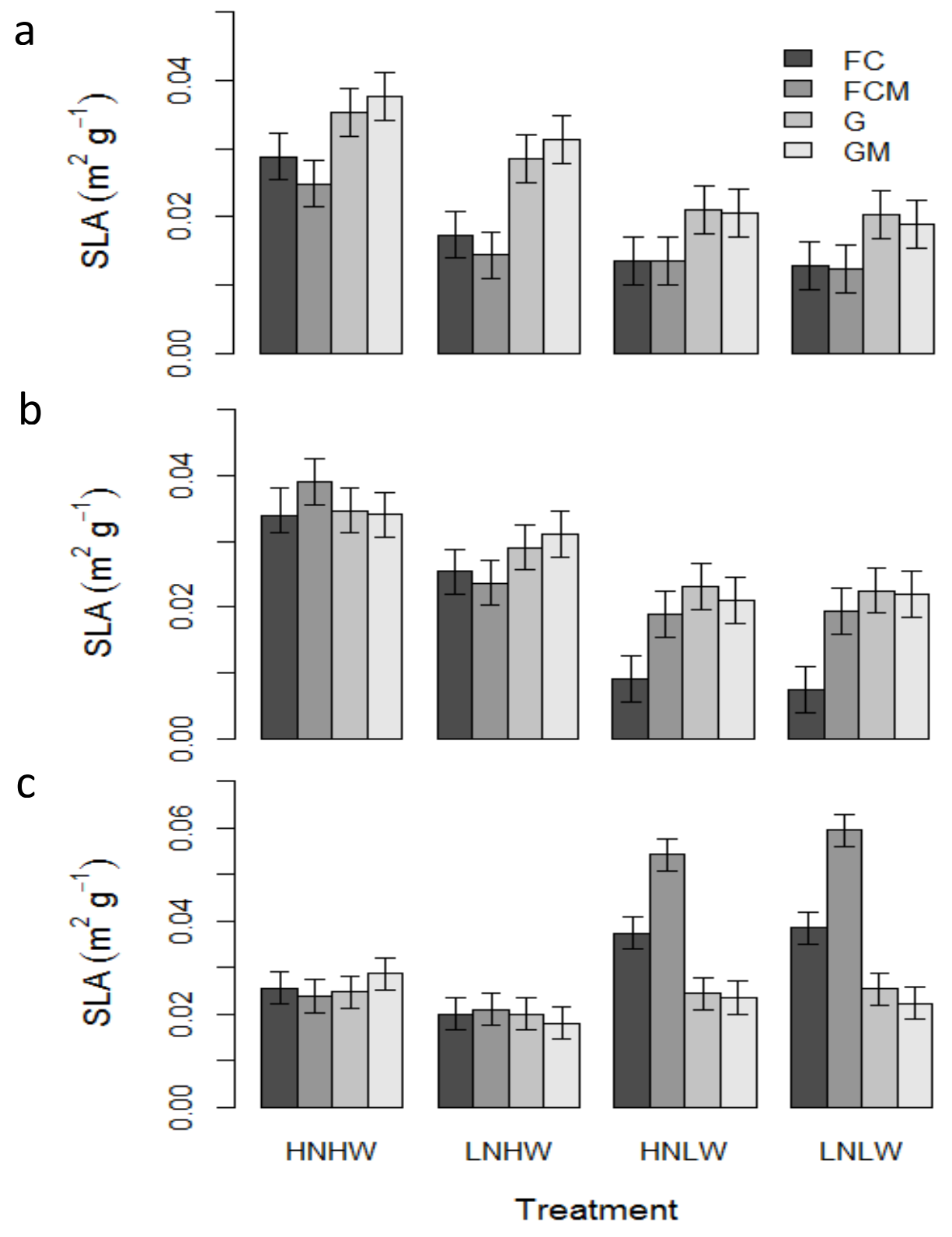

Supplementary Figure 6-2 Specific leaf area (SLA) in CAM alone (FC), CAM in mixture (FCM), grass alone $(\mathrm{G})$, grass in mixture $(\mathrm{GM})$ as affected by nutrient and water conditions in the first (a), second (b), and third (c) stages. Symbols for each treatment are the same as Supplementary Figure 6.1. Each bar represents the mean of 6 values while error bar indicates $95 \%$ confidence intervals. 


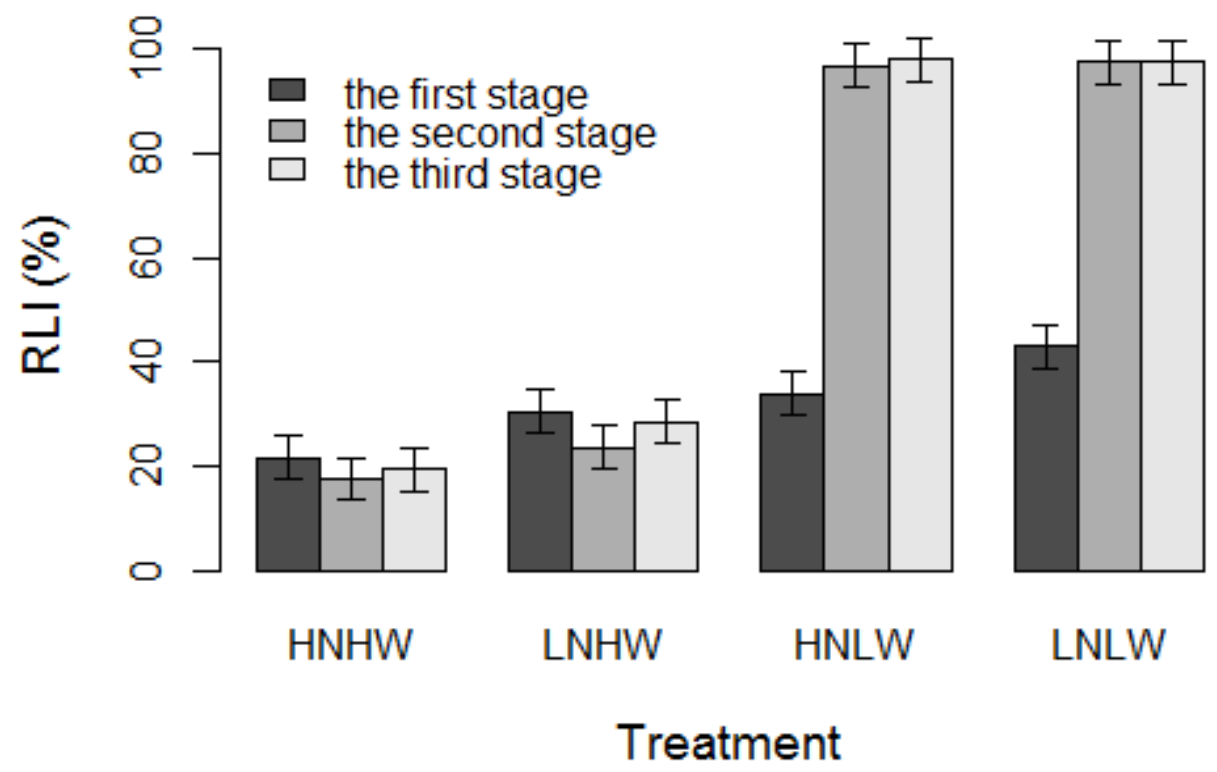

Supplementary Figure 6-3: Relative light intensity (RLI) for CAM in mixture as affected by nutrient and water conditions in the first, second, and third stages. Symbols for each treatment are the same as Supplementary Figure 6.1. Each bar represents the mean of 6 values while error bar indicates $95 \%$ confidence intervals. 
a

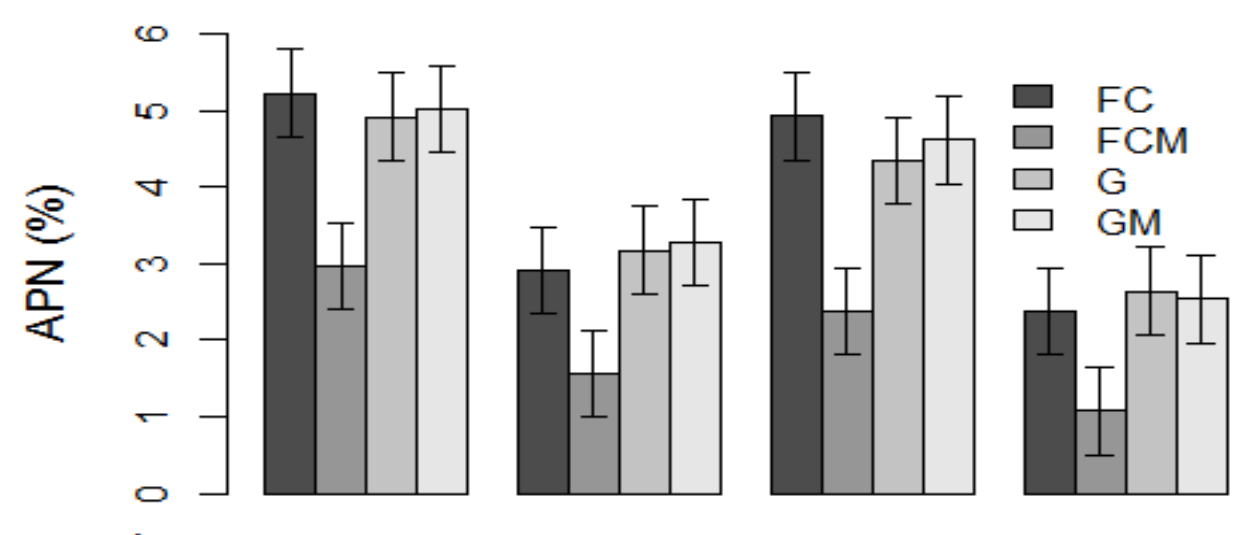

b

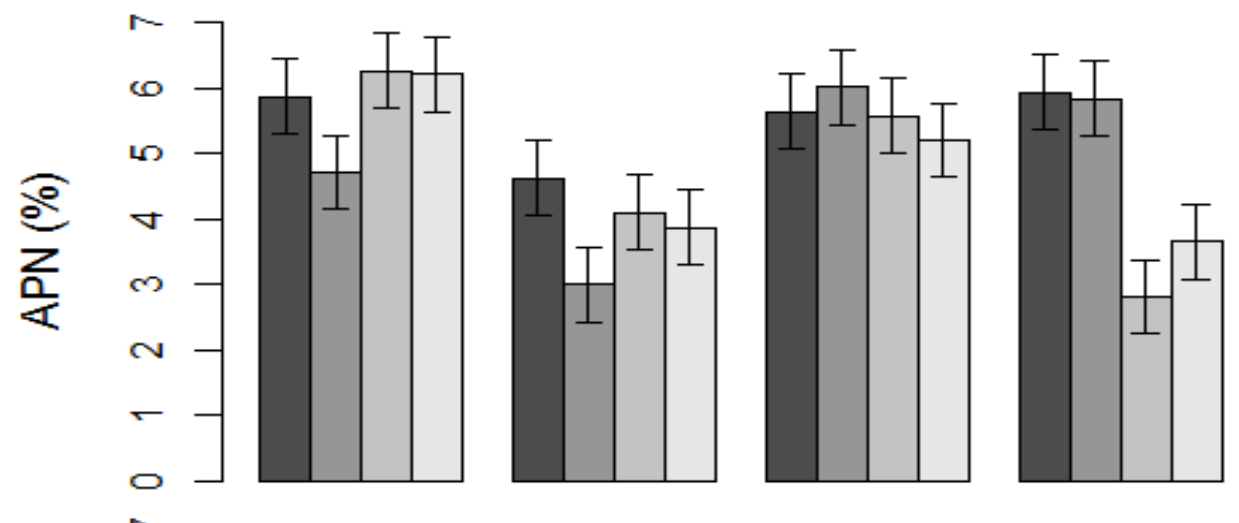

C

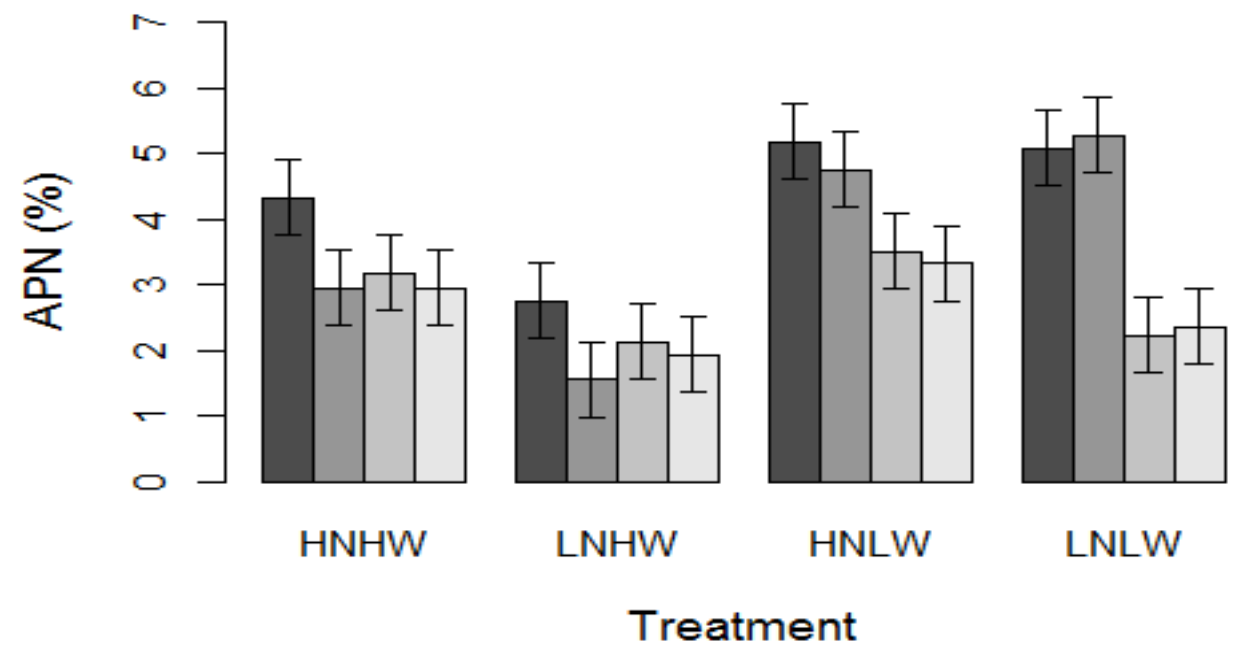

Supplementary Figure 6-4: Aboveground plant nitrogen (APN) in CAM alone (FC), CAM in mixture (FCM), grass alone (G), grass in mixture (GM) as affected by nutrient and water conditions in the first (a), second (b), and third (c) stages. Symbols for each treatment are the same as Supplementary Figure 6.1. Each bar represents the mean of 6 values while error bar indicates $95 \%$ confidence intervals. 


\section{A6 Hydraulic redistribution in tree-grass, CAM-grass, and tree-CAM associations: the implications of Crassulacean Acid Metabolism (CAM)}

\section{$\underline{\text { Supplementary Information }}$}

Supplementary Table 7-1: Parameters, parameter values, and reference sources used in the model.

\begin{tabular}{llll}
\hline Parameter & Symbol Vefence
\end{tabular}

Factor reducing root hydraulic conductance

Extinction coefficient of shortwave radiation

Storage conductance per unit leaf area

Plant conductance per unit leaf area

Atmospheric conductance

Stomatal conductance for $\mathrm{CO}_{2}$

Total root area index

Total moles of solute per unit leaf area

Maximum water storage capacitance

Parameters used to fit the pressure volume curves

Atmospheric pressure c

$$
\frac{1}{1+\frac{\max \left(\Psi_{S 2}, \Psi_{S 1}\right)^{b}}{\Psi_{50}}}
$$

Ryel et al [2002]

$k_{s} \quad 0.35$

Brutsaert [1982]

$g_{c} \quad 0.002 \mathrm{um} \mathrm{Mpa}^{-1} \mathrm{~s}^{-1}$

Bartlett et al [2014]

$g_{p} \quad 0.04 \mathrm{um} \mathrm{Mpa}^{-1} \mathrm{~s}^{-1}$

Bartlett et al [2014]

$g_{a} \quad 0.029 \mathrm{~m} \mathrm{~s}^{-1}$

Bartlett et al [2014]

$g_{s C} \quad 0.003 \mathrm{~m} \mathrm{~s}^{-1}$

Bartlett et al [2014]

RAI $\quad 3 \mathrm{~m}^{2} \mathrm{~m}^{-2}$

Larcher [2003]

$n_{s} \quad 1.36 \mathrm{~mol} \mathrm{~m}^{-2}$

Bartlett et al [2014]

$D_{\max }$

$4.15 \mathrm{~mm}$

Bartlett et al [2014]

$\theta_{1} \quad 0.028$

Bartlett et al [2014]

$\theta_{2} \quad 8$

Bartlett et al [2014]

$p_{a} \quad 101300 \mathrm{~Pa}$

Bartlett et al [2014] 
Air density

$\begin{array}{ll}\rho_{a} & 1.2 \mathrm{Kg} \mathrm{m}^{-3} \\ a_{\text {sat }} & 613.75 \\ b_{\text {sat }} & 17.502 \\ c_{\text {sat }} & 240.97\end{array}$

Bartlett et al [2014]

Jones (1992)

Jones (1992)

Jones (1992)

Latent heat of vaporization

$\lambda_{w} \quad 2500000 \mathrm{~J} \mathrm{Kg}^{-1}$

Bartlett et al [2014]

Air temperature for dry environment at night

$T_{a} \quad 293 \mathrm{~K}$

This study

Air temperature for relatively wet environment at

$293 \mathrm{~K}$

This study night $\quad T_{a}$

Specific humidity in the atmosphere for dry environment

$0.00359 \mathrm{Kg} \mathrm{Kg}^{-1} \quad$ This study

$q_{a}$

Specific humidity in the atmosphere for

relatively wet

$0.00504 \mathrm{Kg} \mathrm{Kg}^{-1} \quad$ This study

environment

$q_{a}$

Relative humility for dry environment at night

RH $\quad 25 \%$

This study

Relative humility for relatively wet environment at

$35 \%$

This study

night $\quad R H$

$\Psi_{50}$ the soil water potential where soil-root conductance is reduced by $50 \%$ and $b$ an empirical constant. $\Psi_{50}=-1 \mathrm{MPa}$ and $b=3.22$ (Ryel et al. 2002). 

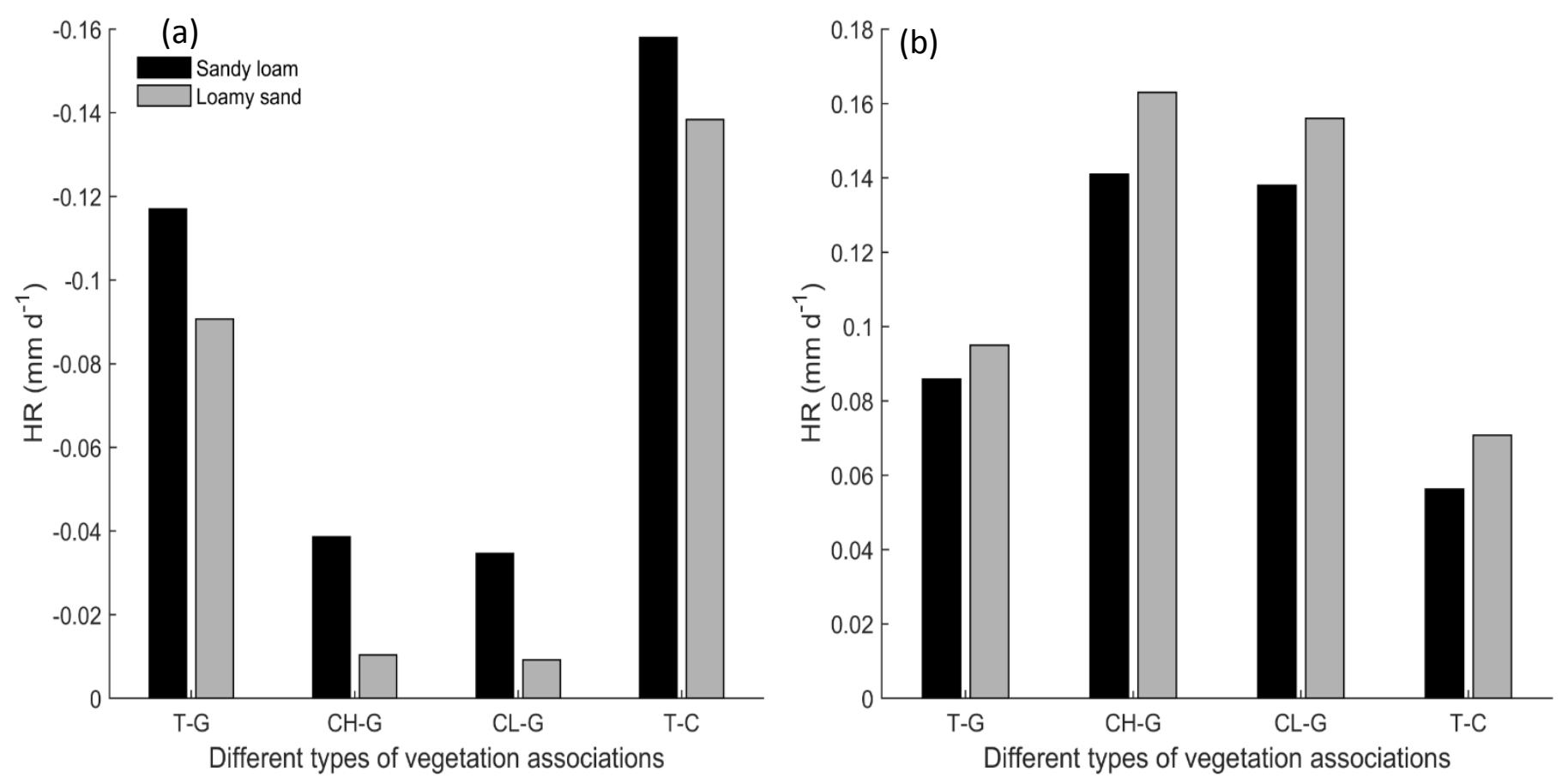

Supplementary Figure 7-1: Hydraulic redistribution (HR) as affected by different types of vegetation associations with $\mathrm{h}=10 \mathrm{~mm}, \lambda=0.1 \mathrm{~d}^{-1}$ (a) and $\lambda=0.2 \mathrm{~d}^{-1}$ (b) for the case of a low mesophyll conductance $\left(\mathrm{g}_{\mathrm{m}}=0.0015 \mathrm{~m} \mathrm{~s}^{-1}\right)$. The symbols are the same as Figure $7.2(\mathrm{a}, \mathrm{b})$ in the main text. 

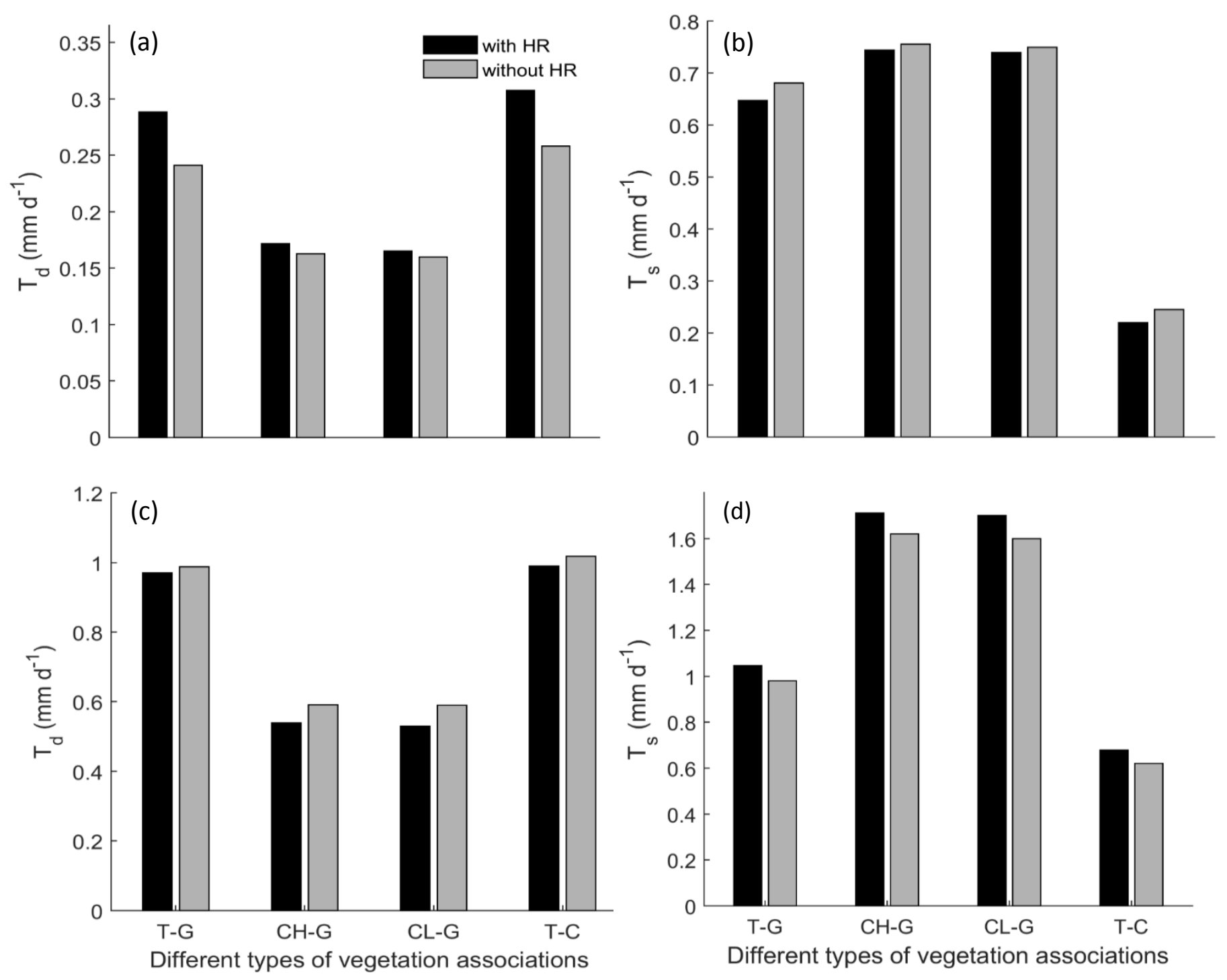

Supplementary Figure 7-2: $(a, b)$ Actual transpiration by deep-root plants $\left(T_{d}\right)(a)$ and shallowrooted plants $\left(\mathrm{T}_{\mathrm{s}}\right)$ (b) as affected by different types of vegetation associations for sandy loam with $\lambda=0.1 \mathrm{~d}-1, \mathrm{~h}=10 \mathrm{~mm}$. (c, d) Actual transpiration by deep-root plants $\left(\mathrm{T}_{\mathrm{d}}\right)$ and shallowedrooted plants $\left(T_{s}\right)(d)$ as affected by different types of vegetation associations with $\lambda=0.2 \mathrm{~d}^{-1}, h$ $=10 \mathrm{~mm}$ for sandy loam. These results are for the case of a high mesophyll conductance $\left(\mathrm{g}_{\mathrm{m}}=\right.$ $\left.0.003 \mathrm{~m} \mathrm{~s}^{-1}\right)$. 


\section{A7 Direct and indirect facilitation of plants with Crassulacean Acid Metabolism (CAM)}

\section{$\underline{\text { Supplementary Information }}$}

Supplementary Table 8-1: Parameters, parameter values, and reference sources used in the study.

\begin{tabular}{llll}
\hline Parameter & Symbol & Value & Reference
\end{tabular}

Maximum total potential evapotranspiration in the $E T_{\operatorname{maxd}} \quad 4.5 \mathrm{~mm} \mathrm{~d}^{-1}$

This study

daytime

Total potential evaporation at soil surface at night

Extinction coefficient of shortwave radiation

Leaf area index of woody plants in arid environment

Leaf area index of woody plants in semiarid environment

Storage conductance per unit leaf area

Leaf area index of CAM plants in arid environment

Leaf area index of CAM plants in semiarid environment

Plant conductance per unit leaf area

Fraction of plant resistance below the storage branch connection

Air density

Specific humidity in the atmosphere in arid environment
$E_{\operatorname{maxn}} \quad 0.5 \mathrm{~mm} \mathrm{~d}^{-1} \quad$ This study

$k_{s} \quad 0.35$

Brutsaert (1982)

$L A I_{t} \quad 1.5 \mathrm{~m}^{2} \mathrm{~m}^{-2}$

This study

$L A I_{t} \quad 3 \mathrm{~m}^{2} \mathrm{~m}^{-2}$

This study

$g_{c} \quad 0.002 \mathrm{um} \mathrm{Mpa}^{-1} \mathrm{~s}^{-1}$

Bartlett and others (2014)

$L A I_{C} \quad 1 \mathrm{~m}^{2} \mathrm{~m}^{-2}$

This study

$L A I_{c} \quad 2 \mathrm{~m}^{2} \mathrm{~m}^{-2}$

This study

$g_{p} \quad 0.0004 \mathrm{um} \mathrm{Mpa}^{-1} \mathrm{~s}^{-1}$

Calkin and Nobel (1986)

f $\quad 0.5$

Bartlett and others (2014)

$\rho_{a} \quad 1.2 \mathrm{Kg} \mathrm{m}^{-3}$

Bartlett and others (2014)

$q_{a} \quad 0.00359 \mathrm{Kg} \mathrm{Kg}^{-1} \quad$ This study 
environment

Factor reducing root hydraulic conductance ${ }^{1}$

$c$

$$
\frac{1}{1+\frac{\max \left(\Psi_{S 2}, \Psi_{S 1}\right)^{b}}{\Psi_{50}}} \text { Ryel and others (2002) }
$$

${ }^{1}: \Psi_{50}$ is the soil water potential where soil-root conductance is reduced by $50 \%$ and $b$ an empirical constant. $\Psi_{50}=-1 \mathrm{MPa}$ and $b=3.22$ (Ryel and others 2002).

Supplementary Table 8-2: Parameters, parameter values, and reference sources used in the study.

\begin{tabular}{lccccccccc}
\hline Soil types & $\Psi_{S}(\mathrm{MPa})$ & $\mathrm{d}$ & $K_{S}\left(\mathrm{~mm} \mathrm{~h}^{-1}\right)$ & $\mathrm{n}$ & $\beta$ & $S_{h}$ & $S_{w}$ & $S^{*}$ & $S_{f c}$ \\
& & & & & & & & & \\
\hline Sandy loam & $-2.1 \times 10^{-3}$ & 4.9 & 33.33 & 0.43 & 13.8 & 0.14 & 0.18 & 0.46 & 0.56 \\
& & & & & & & & & \\
Loamy sand & $-0.88 \times 10^{-3}$ & 4.38 & 50 & 0.42 & 12.76 & 0.08 & 0.11 & 0.33 & 0.35
\end{tabular}

The values of these parameters are from Laio and others (2001). Following Laio and others (2001), $\beta$ is calculated as $\beta=2 \times d+4$. 


\section{Bibliography}

Adams P, Nelson DE, Yamada S, Chmara W, Jensen RG, Bohnert HJ, Griffiths H. 1998. Growth and development of Mesembryanthemum crystallinum (Aizoaceae). New Phytologist 138: 171190.

Ainsworth EA, Long SP. 2005. What have we learned from 15 years of free-air $\mathrm{CO}_{2}$ enrichment (FACE)? A meta-analytic review of the responses of photosynthesis, canopy properties and plant production to rising $\mathrm{CO}_{2}$. New Phytologist 165: 351-372.

Alvarez L, Epstein HE, Li J, Okin GS. 2012. Aeolian process effects on vegetation communities in an arid grassland ecosystem. Ecology and Evolution 2: 809-821.

Anderegg WRL, Kane J, Anderegg LDL. 2013. Consequences of widespread tree mortality triggered by drought and temperature stress. Nature Climate Change 3: 30-36.

Anderegg WRL, Schwalm C, Biondi F, Camarero JJ, Koch G, Litvak M, Ogle K, Shaw JD, Shevliakova E, Williams AP, Wolf A, Ziaco E, Pacala S. 2015. Pervasive drought legacies in forest ecosystems and their implications for carbon cycle models. Science 349: 528-532. Anderies JM, Janssen MA, Walker BH. 2002. Grazing, management, resilience and the dynamics of fire-driven rangeland system, Ecosystems 5: 23-44.

Anderson EF. 2001. The cactus family. Pentland. Oregon: Timber Press.

Anderson LJ, Maherali H, Johnson HB, Wayne Polley H. 2001. Gas exchange and photosynthetic acclimation over sub ambient to elevated $\mathrm{CO} 2$ in a $\mathrm{C}_{3}-\mathrm{C}_{4}$ grassland. Glob Change Biology. 7: 693-707.

Anthelme F, Michalet R. 2009. Grass-to-tree facilitation in an arid grazed environment (Aïr Mountains, Sahara). Basic Applition Ecology 10: 437-446. 
Antony E, Borland AM. 2008. The role and regulation of sugar transporters in plants with Crassulacean acid metabolism. Progress in Botany 70: 127-143.

Antony E, Taybi T, Courbot M, Mugford S, Smith JAC, Borland AM. 2008. Cloning, localisation and expression analysis of vacuolar sugar transporters in the CAM plant Ananas comosus (pineapple). Journal of Experimental Botany 59: 1895-1908.

Archer S. 1989. Have southern Texas savannas been converted to woodlands in recent history? American Naturalist 134: 545-561.

Archer S., Schimel DS, Holland EA. 1995. Mechanisms of shrubland expansion: land use, climate or $\mathrm{CO}_{2}$. Climatic Change 29: 91-99.

Armas C, Kim JH, Bleby TM, Jackson RB. 2012. The effect of hydraulic lift on organic matter decomposition, soil nitrogen cycling, and nitrogen acquisition by a grass species. Oecologia 168 : $11-22$.

Bacilio M, Vazquez P, Bashan Y. 2011. Water versus spacing: A possible growth preference among young individuals of the giant cardon cactus of the Baja California Peninsula.

Environmental Experiment Botany 70: 29-36.

Baez S, Collins SL, Pockman WT, Johnson JE, Small EE. 2013. Effects of experimental rainfall manipulation on Chihuahuan Desert grassland and shrubland plant communities. Oecologia 172: $1117-1127$.

Baker I, Prihodko L, Denning A, Goulden M, Miller S, Da Rocha H. 2008. Seasonal drought stress in the Amazon: Reconciling models and observations. Journal of Geophysic Research 113: G00B01. 
Bakker JP, Bakker ES, Rosén E, Verweij GL, Bekker RM. 1996. Soil seed bank composition along a gradient from dry alvar grassland to Juniperus shrubland. Journal of Vegetation Science 7: $165-176$.

Barbier N, Couteron P, Lefever R, Deblauwe V, Lejeune O. 2008. Spatial decoupling of facilitation and competition at the origin of gapped vegetation patterns. Ecology 89: 1521-1531. Bartlett M, Vico G, Porporato A. 2014. Coupled carbon and water fluxes in CAM photosynthesis: modeling quantification of water use efficiency and productivity. Plant and Soil 383: $111-138$.

Beckage B, Platt WJ, Gross LJ.2009. Vegetation, fire, and feedbacks: a disturbance-mediated model of savannas, American Naturalist 174(6): 805-818.

Bekker RM, Bakker JP, Grandin U, Kalamees R, Milberg P, Poschlod P, Thompson K, Willems JH. 1998. Seed size, shape and vertical distribution in the soil: indicators of seed longevity. Functional Ecology 12: $834-842$.

Benedetti-Cecchi L, Bertocci I, Vaselli S, Maggi E. 2006. Temporal variance reverses the impact of high mean intensity of stress in climate change experiments. Ecology 87: 2489-2499.

Beyers JL. 2004. Postfire seeding for erosion control: effectiveness and impacts on native plant communities. Conservation Biology 18(4): 947-956.

Bhattachan A, D’Odorico P, Dintwe K, Okin GS, Collins SL. 2014. Resilience and recovery of the Kalahari dunes. Ecosphere 5(2): 1-14.

Bhattachan A, Tatlhego M, Dintwe K, O’Donnell FC, Caylor KK, Okin GS, Perrot DO, Ringrose S, D’Odorico P. 2012. Ecohydrologic controls on root biomass and depth in southern African savannas. PLoS One 7:e33996. 
Binggeli, P.1996. A taxonomic, biogeographical and ecological overview of invasive woody plants. Journal of Vegetation Science 7: 121-124.

Bond WJ. 2008. What limits trees in $\mathrm{C}_{4}$ grasslands and savannas? Annual Review of Ecology, Evolution, and Systematics 39: 641-659.

Bond WJ, Woodward FI, Midgley GF. 2005. The global distribution of ecosystems in a world without fire. New Phytologist 165: 525-537.

Borland AM, Barrera Zambrano VA, Ceusters J, Shorrock K. 2011. The photosynthetic plasticity of crassulacean acid metabolism: an evolutionary innovation for sustainable productivity in a changing world. New Phytologist 191: 619-633.

Borland AM, Dodd AN. 2002. Carbohydrate partitioning in crassulacean acid metabolism plants: reconciling potential conflicts of interest. Functional Plant Biology 29: 707-716.

Borland AM, Griffiths H, Hartwell J, Smith JAC. 2009. Exploiting the potential of plants with crassulacean acid metabolism for bioenergy production on marginal lands. Journal of Experimental Botany 60: 2879-2896.

Borland AM, Wullschleger SD, Weston DJ, Hartwell J, Tuskan GA, et al. 2015. Climateresilient agroforestry: physiological responses to climate change and engineering of crassulaceanacid metabolism (CAM) as a mitigation strategy. Plant Cell \& Environment 38: 1833-49.

Boulant N, Navas M-L, Corcket E, Lepart J. 2008. Habitat amelioration and associational defence as main facilitative mechanisms in Mediterranean grasslands grazed by domestic livestock. Ecoscience 15: 407-415. 
Boulet G, Chehbouni A, Braud I, Vauclin M, Haverkamp R, Zammit C. 2000. A simple water and energy balance model designed from regionalization and remote sensing data utilization. Agricultural and Forest Meteorology 105: 117-132.

Bragg WK, Knapp AK, Briggs JM. 1993. Comparative water relations of seedling and adult Quercus species during gallery forest expansion in tallgrass prairie. Forest Ecology and Management 56: 29-41.

Breman H, Cissé AM. 1977. Dynamics of sahelian pastures in relation to drought and grazing. Oecologia 28: 301-315.

Brooker RW, Maestre FT, Callaway RM, Lortie CL, Cavieres LA, Kunstler G, Liancourt P, Tielbörger K, Travis JM, Anthelme F. 2008. Facilitation in plant communities: the past, the present, and the future. Journal of Ecology 96: 18-34.

Brooks JR, Meinzer FC, Warren JM, Domec J-C, Coulombe R. 2006. Hydraulic redistribution in a Douglas-fir forest: lessons from system manipulations. Plant Cell \& Environment 29: 138-150. Brown JR, Archer S. 1990. Water relations of a perennial grass and seedling vs adult woody plants in a subtropical savanna, Texas. Oikos 57: 366-374.

Brown JH, Valone TJ, Curtin CG. 1997. Reorganization of an arid ecosystem in response to recent climate change. Proceedings of the National Academy of Sciences of the United States of America 94: 9729-33.

Bruno JF, Stachowicz JJ, Bertness MD. 2003. Inclusion of facilitation into ecological theory. Trends in Ecology and Evolution 18: 119-125.

Brutsaert W. 1982. Evaporation into the Atmosphere: Theory,. History and Applications, D. Reidel Publishing Company, Boston. 
Buffington LC, Herbel CH. 1965. Vegetational changes on a semi desert grassland range from 1858 to 1963. Ecological Monographs 35: 139-164.

Burgess SS, Adams MA, Turner NC, White DA, Ong CK. 2001. Tree roots: conduits for deep recharge of soil water. Oecologia 126: 158-165.

Burrows WH, Carter JO, Scanlan JC, Anderson ER. 1990. Management of savannas for livestock production in north-east Australia - contrasts across the tree grass continuum. Journal of Biogeography 17: 503-512.

Callaway RM. 2007. Positive interactions and interdependence in plant communities: Springer. Callaway RM, Brooker R, Choler P, Kikvidze Z, Lortie CJ, Michalet R, Paolini L, Pugnaire FI, Newingham B, Aschehoug ET. 2002. Positive interactions among alpine plants increase with stress. Nature 417: 844-848.

Caldwell MM, Dawson TE, Richards JH. 1998. Hydraulic lift: consequences of water efflux from the roots of plants. Oecologia 113:151-161.

Campbell GS, Norman JM. 1998. An introduction to environmental biophysics, Springer-Verlag, New York.

Cares RA, Muñoz PA, Medel R, Botto-Mahan C. 2013. Factors affecting cactus recruitment in semiarid Chile: A role for nurse effects? Flora 208: 330-335.

Casper BB, Jackson RB. 1997. Plant competition underground. Annual Review of Ecology, Evolution, and Systematics 28: 545-570.

Casper BB, Schenk HJ, Jackson RB. 2003. Defining a plant's belowground zone of influence. Ecology 84: 2113-2321.

Castillo JP, Valiente-Banuet A. 2010. Species-specificity of nurse plants for the establishment, survivorship, and growth of a columnar cactus. American Journal of Botany 97: 1289-1295. 
Caylor KK, D'Odorico P, Rodriguez-Iturbe I. 2006. On the ecohydrology of structurally heterogeneous semiarid landscapes, Water Resource Research 42: W07424.

Caylor KK, Shugart HH, Rodriguez-Iturbe I. 2005. Tree canopy effects on simulated water stress in southern African savannas. Ecosystems 8: 17-32.

Ceusters J, Borland AM, Godts C, Londers E, Croonenborghs S, Van Goethem D, Maurice P. 2011. Crassulacean acid metabolism under severe light limitation: a matter of plasticity in the shadows? Journal of Experimenatal Botany 62: 283-291.

Chidumayo EN. 2004. Development of Brachystegia-Julbernardia woodland after clear felling in central Zambia: evidence for high resilience. Applied Vegetation Science 7: 237-242.

Clapp RB, Hornberger GM. 1978. Empirical equations for some soil hydraulic properties. Water Resource Research 14: 601-604.

Cochrane MA. 2003. Fire science for rainforests. Nature 421: 913-919.

Collins, SL, Koerner SE, Plaut JA, Okie JG, Brese D, Calabrese LB, Carvajal A, Evansen RJ, Nonaka E. 2012. Stability of tallgrass prairie during a 19-year increase in growing season precipitation. Functional Ecology 26: 1450-1459.

Collins SL, Belnap J, Dahm CN, et al. 2014. A multi-scale, hierarchical model of pulse dynamics in aridland ecosystems. Annual Review of Ecology, Evolution, and Systematics 45: 397-419.

Collins SL, Xia Y. 2015. Long-term dynamics and hotspots of change in a desert grassland plant community. American Naturalist 185: E30-E43.

Connell JH. 1983. On the prevalence and relative importance of interspecific competition: evidence from field experiments. American Naturalist. 122: 661-696. 
Coomes DA, Allen RB. 2007. Effects of size, competition and altitude on tree growth. Journal of Ecology 95: 1084-1097.

Corbin JD, D’Antonio CM. 2004. Competition between native perennial and exotic annual grasses: implications for an historical invasion. Ecology 85: 1273-1283.

Corcket E, Callaway RM, Michalet R. 2003. Insect herbivory and grass competition in a calcareous grassland: results from a plant removal experiment. Acta Oecologia 24: 139-146. Cuesta B, Villar-Salvador P, Puertolas J, Rey Benayas JM, Michalet R. 2010. Facilitation of Quercus ilex in Mediterranean shrubland is explained by both direct and indirect interactions mediated by herbs. Journal of Ecology 98: 687-696.

Cushman JC, Agarie S, Albion RL, Elliot SM, Taybi T, Borland AM. 2008. Isolation and characterization of mutants of common ice plant deficient in crassulacean acid metabolism. Plant Physiology 147: 228-238.

Cushaman JC, Borland AM. 2002. Induction of crassulacean acid metabolism by water limitation. Plant Cell and Environment 25: 295-310.

Dai AG. 2010. Drought under global warming: a review. Wiley Interdisc. Rev.Clim. Change 2: $45-65$.

D'Antonio CM. 2000. Fire, plant invasions, and global change, Pages 65-93 in H. A. Mooney, and R. J. Hobbs, eds. Invasive Species in a Changing World. Washington, D.C., Island Press. D'Antonio CM, Vitousek PM. 1992. Biological invasions by exotic grasses, the grass/fire cycle, and global change. Annual Review of Ecology, Evolution, and Systematics 23: 63-87.

Dantas VL, Batalha MA, Pausas JG. 2013. Fire drives functional thresholds on the savannaforest transition, Ecology 94: 2454-2463. 
Dauber E, Fredericksen, T.S., M. Peña-Claros. 2005. Sustainability of timber harvesting in Bolivian tropical forests. Forest Ecology and Management 214: 294-304.

Davidson. 1999. Positive feedbacks in the fire dynamic of closed canopy tropical forests. Science 284: 1832-1835.

Dawson TE. 1993. Hydraulic lift and water use by plants: implications for water balance, performance and plant-plant interactions. Oecologia 95: 565-574.

Davis SC, Dohleman FG, Long SP. 2011. The global potential for Agave as a biofuel feedstock. GCB Bioenergy 3: 68-78.

Davis MA, Grime JP, Thompson K. 2000. Fluctuating resources in plant ommunities: a general theory of invisibility. Journal of Ecology 88: 528-534.

Davis MA, Pelsor M. 2001. Experimental support for a resource-based mechanistic model of invasibility. Ecology Letters 4: 421-428.

Dawson TE. 1993. Hydraulic lift and water use by plants: implications for water balance, performance and plant-plant interactions. Oecologia 95: 565-574.

De La Barrera E, Smith WK. 2009. Perspectives in Biophysical Plant Ecophysiology: A Tribute to Park S. Nobel: UNAM.

D’Odorico P, Ridolfi L, Porporato A, Rodriguez-Iturbe I. 2000. Preferential states of seasonal soil moisture: The impact of climate fluctuations. Water Resources Research 36: 2209-2219. D'Odorico P, Caylor K, Okin GS, Scanlon TM. 2007. On soil moisture-vegetation feedbacks and their possible effects on the dynamics of dryland ecosystems. Journal of Geophysic Research 112: G04010.

D’Odorico P, Laio F, Porporato A, Rodriguez-Iturbe I. 2003. Hydrologic controls of soil carbon and nitrogen cycles II. A case study. Advance in Water Resources 26: 59-70. 
D'Odorico P, Laio F, Porporato A, Ridolfi L, Barbier N. 2007. Noise-induced vegetation patterns in fire-prone savannas. Journal of Geophysical Research, 112, G02021.

D'Odorico P, Laio F, Ridolfi L. 2005. Noise-induced stability in dryland plant ecosystems. Proceedings of the National Academy of Sciences of the United States of America. 102: 1081910822.

D'Odorico P, Laio F, Ridolfi L. 2006a. A probabilistic analysis of fire induced tree-grass coexistence in savannas. American Naturalist 167: E79-E87.

D'Odorico P, Laio F, Ridolfi L. 2006b. Vegetation patterns induced by random climate fluctuations. Geophysical Research Letter 33: L19404.

D'Odorico P, Laio F, Ridolfi L, Lerdau MT. 2008. Biodiversity enhancement induced by environmental noise. Journal of Theoretical Biology 255: 332-337.

D’Odorico P, Okin GS, Bestelmeyer BT. 2012. A synthetic review of feedbacks and drivers of shrub encroachment in arid grasslands, Ecohydrology 5: 520-530.

Dohn J, Dembélé F, Karembé M, Moustakas A, Amévor KA, Hanan NP. 2013. Tree effects on grass growth in savannas: Competition, facilitation and the stress-gradient hypothesis. Journal of Ecology 101: 202-209.

Domec JC, King JS, Noormets A, Treasure E, Gavazzi MJ, Sun G, McNulty SG. 2010.

Hydraulic redistribution of soil water by roots affects whole-stand evapotranspiration and net ecosystem carbon exchange. New Phytologist. 187: 171-183. 
Domec JC, Warren J, Meinzer F, Brooks J, Coulombe R. 2004. Native root xylem embolism and stomatal closure in stands of Douglas-fir and ponderosa pine: mitigation by hydraulic redistribution. Oecologia 141: 7-16.

Drennan PM, Nobel PS. Responses of CAM species to increasing atmospheric $\mathrm{CO}_{2}$ concentrations. Plant Cell Environment 23: 767-781.

Drezner TD. 2007. An analysis of winter temperature and dew point under the canopy of a common Sonoran Desert nurse and the implications for positive plant interactions. Journal of Arid Environment 69: 554-568.

Dublin HT, Sinclair AR, McGlade J. 1990. Elephants and Fire as causes of multiple stable states in the Serengeti-Mara woodlands. Journal of Animal Ecology 59: 1147-1164.

Dukes JS, Mooney HA. 1999. Does global change increase the success of biological invaders? Trends in Ecology and Evolution 14: 135-139.

Eagleson PS, Segarra RI. 1985. Water-limited equilibrium of savanna vegetation systems. Water Resource Research 21: 1483-1493.

Easterling DR, Meehl GA, Parmesan C, Changnon SA, Karl TR, Mearns LO. 2000. Climate extremes: Observations, modeling, and impacts. Science 289: 2068-2074.

Egerton-Warburton LM, Querejeta JI, Allen MF. 2007. Common mycorrhizal networks provide a potential pathway for the transfer of hydraulically lifted water between plants. Journal of Experimental Botany 58:1473-1483.

Ehlers BK, Charpentier A, Grøndahl E. 2014. An allelopathic plant facilitates species richness in the Mediterranean garrigue. Journal of Ecology 102: 176-185. 
Eldridge DJ, Bowker MA, Maestre FT, Roger E, Reynolds JR, Whitford WG. 2011. Impacts of shrub encroachment on ecosystem structure and functioning: towards a global synthesis. Ecology Letters 14: 709-722.

Ellis RH. 1991. The longevity of seeds HortScience. 26: 1119-1125.

Espeleta J, West J, Donovan L. 2004. Species-specific patterns of hydraulic lift in co-occurring adult trees and grasses in a sandhill community. Oecologia 138: 341-349.

Fatichi S, Ivanov V. 2014. Interannual variability of evapotranspiration and vegetation productivity. Water Resources Research 50: 3275-3294.

Fay PA, Jin VL, Way DA, Potter KN, Gill RA, Jackson RB, Polley HW. 2012. Soil-mediated effects of subambient to increased carbon dioxide on grassland productivity. Nature Climate Change 2: 742-746.

Fetene M, Lee H, Lüttge U. 1990. Photosynthetic acclimation in a terrestrial CAM bromeliad, Bromelia humilis Jacq. New Phytologist 114: 399-406.

Flexas J, Ribas-Carbó M, Diaz-Espejo A, Galmés1 J, Medrano H. 2008. Mesophyll conductance to CO2: current knowledge and future prospects. Plant Cell \& Environment 31: 602-621. Flores-Martinez A, Ezcurra E, Sanchez-Colon S. 1994. Effect of Neobuxbaumia tetetzo on growth and fecundity of its nurse plant Mimosa luisana. Journal of Ecology 82: 325-330. Flores-Torres A, Montana. 2015. From facilitative to competitive interaction between Larrea tridentata and Cylindropuntia leptocaulis in the southern Chihuahuan Desert. Journal of Vegetation Science 26: 68-79. 
Franklin KA, Lyons K, Nagler PL, Lampkin D, Glenn EP, Molina-Freaner F, Markow T, Huete AR. 2006. Buffelgrass (Pennisetum ciliare) land conversion and productivity in the plains of Sonora, Mexico. Biological Conservation 127: 62-71.

Garcia-Ojalvo J, Sancho JM. 1999. Noise in Spatially-Extended Systems. Berlin, SpringerVerlag.

Gardner TA. 2006. Tree-grass coexistence in the Brazilian cerrado: demographic consequences of environmental instability. Journal of Biogeography 33:448-463.

Germino MJ, Belnap J, Stark JM, Allen EB, Rau BM. 2016. Exotic Brome-Grasses in Arid and Semiarid Ecosystems of the Western US: Causes, Consequences, and Management Implications. NewYork, Springer.

Germino MJ, Reinhardt K. 2014. Desert shrub responses to experimental modification of precipitation seasonality and soil depth: relationship to the two-layer hypothesis and ecohydrological niche. Journal of Ecology 102: 989-997.

Gherardi, LA, Sala OE. 2015a. Enhanced interannual precipitation variability increases plant functional diversity that in turn ameliorates negative impact on productivity. Ecology Letters 18: 1293-1300.

Gherardi, LA, Sala OE. 2015b. Enhanced precipitation variability decreases grass- and increases shrub-productivity. Proceedings of the National Academy of Sciences of the United States of America 112: 12735-12740.

Graham EA, Nobel PS. Long-term effects of a doubled atmospheric $\mathrm{CO}_{2}$ concentration on the CAM species Agave deserti. Journal of Experimental Botany 47: 61-69. 
Grigulis K, Lavorel S, Davies ID, Dossantos A, Lloret F, M. Vilà. 2005. Landscape-scale positive feedbacks between fire and expansion of the large tussock grass, Ampelodesmos mauritanica in Catalan shrublands. Global Change Biology 11: 1042-1053.

Guralnick LJ, Ting IP. 1987. Physiological changes in Portulacaria afra (L.) Jacq. during a summer drought and rewatering. Plant Physiology 85: 481-486.

Guswa A, Celia MA, Rodriguez-Iturbe I. 2002. Models of soil moisture dynamics in ecohydrology: a comparative study. Water Resource Research 38:1166.

Guswa A, Celia MA, Rodriguez-Iturbe I. 2004. Effect of vertical resolution on predictions of transpiration in water-limited ecosystems. Advance in Water Resource 27: 467-480.

Haider MS, Barnes JD, Cushman JC, Borland AM. 2012. A CAM- and starch-deficient mutant of the facultative CAM species Mesembryanthemum crystallinum reconciles sink demands by repartitioning carbon during acclimation to salinity. Journal of Experimental Botany 63: 19851996.

Hansen MC, DeFries RS, Townshend JRG, Carroll M, Dimiceli C, Sohlberg RA. 2003. Global percent tree cover at a spatial resolution of 500 meters: First results of the MODIS vegetation continuous fields algorithm. Earth Interact 7: 1-15.

Harpole WS, Potts DL, Suding KN. 2007. Ecosystem responses to water and nitrogen amendment in a California grassland. Global Change Biology 13: 2341-2348.

Havstad KM, Gibbens RP, Knorr CA, Murray LW. 1999. Long-term influences of shrub removal and lagomorph exclusion on Chihuahuan Desert vegetation dynamics. Journal of Arid Environment 42: 155-166.

Herbel. 1972. Drought effects on a semidesert grassland range. Ecology 53: 1084-1093. Herrera A. 2009. Crassulacean acid metabolism and fitness under water deficit stress: if not 
for carbon gain, what is facultative CAM good for? Annals of Botany 103: 645-653.

Herzog B, Hoffmann S, Hartung W, Lüttge U. 1999. Comparison of photosynthetic responses of the sympatric tropical $\mathrm{C}_{3}$ species Clusia multiflora H.B.K. and the $\mathrm{C}_{3}-\mathrm{CAM}$ intermediate species Clusia minor L. to irradiance and drought stress in a phytotron. Plant Biology 1: 460-470. Hibbard KA, Archer S, Schimel DS, Valentine DW. 2001. Biogeochemical changes accompanying woody plant encroachment in a subtropical savanna. Ecology 82: 1999-2011. Higgins SI, Scheiter S. 2012. Atmospheric CO2 forces abrupt vegetation shifts locally, but not globally. Nature 488: 209-212.

Higgins SI, Scheiter S, Sankaran M. 2010. The stability of African savannas: insights from the indirect estimation of parameters of a dynamic model. Ecology 91: 1682-1692.

Hipondoka MHT, Aranibar JN, Chirara C, Lihavha M, Macko SA. 2003. Vertical distribution of grass and tree roots in arid ecosystems of southern Africa: niche differentiation or competition? Journal of Arid Environment 54: 319-325.

Hirota M, Holmgren M, Van Nes EH, Scheffer M. 2011. Global resilience of tropical forest and savanna to critical transitions. Science 334: 232-235.

Hoffmann WA, Schroeder W, Jackson RB. 2002. Positive feedbacks of fire, climate, and vegetation and the conversion of tropical savannas. Journal of Geophysical Research 108(D23): 4721.

Hoffmann WA, Geiger EL, Gotsch S, et al. 2012. Ecological thresholds at the savanna-forest boundary: How plant traits, resources and fire govern the distribution of tropical biomes. Ecology Letters 15: 759-768.

Holdo RM. 2006. Tree growth in an African woodland savanna affected by disturbance. Journal of Vegetation Science 1: 369-378. 
Holdo RM. 2013. Revisiting the two-layer hypothesis: coexistence of alternative functional rooting strategies in savannas. PloS One 8: e69625.

Holdo RM, Nippert JB. 2015. Transpiration dynamics support resource partitioning in African savanna trees and grasses. Ecology 96: 1466-1472.

Holmgren M, Hirota M, Van Nes EH, Scheffer M. 2013. Effects of interannual climate variability on tropical tree cover. Nature Climate Change 3: 755-758.

Holzapfel C, Mahall BE. 1999. Bidirectional facilitation and interference between shrubs and annuals in the Mojave Desert. Ecology 80: 1747-1761.

Hooper DU, Johnson L. 1999. Nitrogen limitation in dryland ecosystems: responses to geographical and temporal variation in precipitation. Biogeochemistry 46: 247-293.

Horsthemke W, Lefever R. 1984. Noise-Induced Transitions. Berlin, Springer-Verlag. Horton J, Hart SC (1998) Hydraulic lift: a potentially important ecosystem process. Trends in Ecology and Evolution 13: 232-235.

House JI, Archer S, Breshears DD, Scholes RJ. 2003. Conundrums in mixed woody-herbaceous plant systems. Journal of Biogeography 30: 1763-1777.

Hsu JS, Adler PB. 2014. Anticipating changes in variability of grassland production due to increases in interannual precipitation variability. Ecosphere 5: art58.

Hsu JS, Powell J, Adler PB. 2012. Sensitivity of mean annual primary production to precipitation. Global Change Biology 18: 2246-2255.

Huffman GJ, Bolvin DT, Nelkin EJ et al. 2007. The TRMM multisatellite precipitation analysis. TMPA: Quasi-global, multiyear, combined-sensor precipitation estimates at fine scales. Journal of Hydrometeorology 8: 38-55. 
Hultine KR, Scott R, Cable W, Goodrich D, Williams D. 2004. Hydraulic redistribution by a dominant, warm-desert phreatophyte: Seasonal patterns and response to precipitation pulses. Functional Ecology 18: 530-538.

Hutto RL, McAuliffe JR, Hogan L.1986. Distributional associates of the saguaro (Carnegiea gigantea). The Southwestern Naturalist 31: 469-476.

Huxman TE, Hamerlynck EP, Moore BD, Smith SD, Jordan DN, Zitzer SF, Nowak RS, Coleman JS, Seemann JR. Photosynthetic down-regulation in Larrea tridentata exposed to elevated atmospheric $\mathrm{CO}_{2}$ : Interaction with drought under glasshouse and field (FACE) exposure. Plant Cell and Environment 21: 1153-1161.

Huxman TE, Wilcox BP, Breshears DD, Scott RL, Snyder KA, Small EE, Hultine K, Pockman WT, Jackson RB. 2005. Ecohydrological implications of woody plant encroachment. Ecology, 86: $308-319$.

Idso SB, Aase JK, Jackson RD. 1975. Net radiation—-soil heat flux relations as influenced by soil water content variations. Boundary Layer Meteorology 9: 113-22.

IPCC. 2013. Climate Change 2013: The Physical Science Basis. Working Group I contribution to the IPCC 5th Assessment Report. In. Geneva: IPCC Secretariat Jackson RB, Sperry JS, Dawson TE. 2000. Root water uptake and transport: using physiological processes in global predictions. Trends in Plant Science 5: 482-488.

Jeltsch F, Milton SJ, Dean WRJ, van Rooyen N. 1996. Tree spacing and coexistence in semiarid savannas. Journal of Ecology 84: 583-595.

Jeltsch F, Weber GE, Grimm V. 2000. Ecological buffering mechanisms in savannas: a unifying theory of long-term tree-grass coexistence. Plant Ecology 161: 161-171. 
He Y, D’Odorico P, DeWekker SF. 2014. On the relative importance of climate change and shrub encroachment on nocturnal warming in the Southwestern U.S. International Journal of Climatology 35: 475-480.

Hibbard KA, Archer S, Schimel DS, Valentine DW. 2001. Biogeochemical changes accompanying woody plant encroachment in a subtropical savanna. Ecology 82: 1999-2011. Higgins SI, Bond WJ, Trollope WSW. 2000. Fire, resprouting and variability: a recipe for treegrass coexistence in savanna. Journal of Ecology 88: 213-229.

Karl TR, Trenberth KE. 2003. Modern global climate change. Science 302: 1719-1723.

Keeley JE, Rundel PW. 2005. Fire and the Miocene expansion of C4 grasslands. Ecology Letters 8: 683-690.

Kieft TL, White CS, Loftin SR, Aguilar R, Craig JA, Skaar DA. 1998. Temporal dynamics in soil carbon and nitrogen resources at a grassland-shrubland ecotone. Ecology 79: 671-683.

Kim Y, Eltahir EAB. 2004. Role of topography in facilitating coexistence of trees and grasses within Savannas. Water Resources Research 40: W07505.

Kljun N, Black TA, Griffis TJ et al. 2006. Response of net ecosystem productivity of three boreal forest stands to drought. Ecosystems 9: 1128-1144.

Knapp AK, Fay PA, Blair JM, Collins SL, Smith MD, Carlisle JD, Harper CW, Danner BT, Lett MS, McCarron JK. 2002. Rainfall variability, carbon cycling and plant species diversity in a mesic grassland. Science 298: 2202-2205.

Knapp AK, Briggs JM, Collins SL, Archer SR, Bret-Harte MS, Ewers BE, Peters DP, et al. 2008. Shrub encroachment in North American grasslands: shifts in growth form dominance rapidly alters control of ecosystem carbon inputs. Global Change Biology 14: 615-623. 
Knipe D, Herbel CH. 1966. Germination and growth of some semidesert grassland species treated with aqueous extract from creosotebush. Ecology: 775-781.

Knoop WT, Walker BH. 1985. Interactions of woody and herbaceous vegetation in a Southern African savanna. Journal of Ecology 73: 235-253.

Koch, G. W., R. J. Scholes, W. L. Steffen, P. M. Vitousek, and B. H. Walker (1995), The IGBP terrestrial transects: Science plan, Report No. 36. International Geosphere-Biosphere Programme, Stockholm, p 61.

Kort J, Collins M, Ditsch D. 1998. A review of soil erosion potential associated with biomass crops. Biomass Bioenergy 14: 351-359.

Kos M, Baskin CC, Baskin JM. 2012. Relationship of kinds of seed dormancy with habitat and life history in the Southern Kalahari flora. Journal of Vegetation Science 23: 869-879.

Kulmatiski A, Beard K. 2013a. Woody plant encroachment facilitated by increased precipitation intensity. Nature Climate Change 3: 833-837.

Kulmatiski A, Beard K. 2013b. Root niche partitioning among grasses, saplings, and trees measured using a tracer technique. Oecologia 171: 25-37.

Kunstler G, Curt T, Bouchaud M, Lepart J. 2006. Indirect facilitation and competition in tree species colonization of sub-Mediterranean grasslands. Journal of Vegetation Science 17: 379388.

Kustas WP, Norman JM. 1997. A two-source approach for estimating turbulent fluxes using multiple angle thermal infrared observations, Water Resources Research 33: 1495-1508. Lai C-T, Katul G. 2000. The dynamic role of root-water uptake in coupling potential to actual transpiration. Advance in Water Resources 23: 427-439. 
Laio F, Porporato A, Ridolfi L, Rodriguez-Iturbe I. 2001. Plants in water-controlled ecosystems: active role in hydrologic processes and response to water stress. II. Probabilistic soil moisture dynamics. Advances in Water Resources 23: 707-723.

Lane DR, Coffin DP, Lauenroth WK. 2000. Changes in grassland canopy structure across a precipitation gradient. Journal of Vegetation Science 11: 359-368.

Lawlor LR. 1979. Direct and indirect effects of n-species competition. Oecologia 43: 355-364. Lee J-E, Oliveira RS, Dawson TE, Fung I. 2005. Root functioning modifies seasonal climate. Proceedings of the National Academy of Sciences of the United States of America 102: 1757617581.

Lehmann CER, Archibald SA, Hoffmann WA, Bond WJ. 2011. Deciphering the distribution of the savanna biome. New Phytologist 191: 197-209.

Lenoir J, Gégout JC, Guisan A, Vittoz P, Wohlgemuth T, Zimmermann NE, Dullinger S, Pauli H, Willner W, Svenning JC. 2010. Going against the flow: potential mechanisms for unexpected downslope range shifts in a warming climate. Ecography 33: 295-303.

Levine JM. 1999. Indirect facilitation: evidence and predictions from a riparian community. Ecology 80: 1762-1769.

Lhomme JP, Monteny B. 2000. Theoretical relationship between stomatal resistance and surface temperatures in sparse vegetation. Agricultural and Forest Meteorology 104: 119-131.

Lhomme J-P, Rocheteau A, Ourcival J, Rambal S. 2001. Non-steady-state modelling of water transfer in a Mediterranean evergreen canopy. Agricultural and Forest Meteorology 108: 67-83. Liang E, Piao S, Lu X, Camarero JJ, Zhu H, Zhu L, Ellison AM, Ciais P, Peñuelas J. 2016. Species interactions slow warming-induced upward shifts of tree lines on the Tibetan Plateau. 
Proceedings of the National Academy of Sciences of the United States of America 113: 43804385.

Liedloff AC, Cook GD. 2007. Modelling the effects of rainfall variability and fire on tree populations in an Australian tropical savanna with the FLAMES simulation model. Ecological Modeling 201: 269-282.

Ludwig F, de Kroon H, Prins HHT, Berendse F. 2001. Effects of nutrients and shade on treegrass interactions in an east African savanna, Journal of Vegetation Science 12: 579-588.

Ludwig F, Dawson TE, de Kroon H, Berendse F, Prins HH. 2003. Hydraulic lift in Acacia tortilis trees on an East African savanna. Oecologia 134: 293-300.

Ludwig F, Dawson T, Prins H, Berendse F, Kroon H. 2004. Below-ground competition between trees and grasses may overwhelm the facilitative effects of hydraulic lift. Ecology Letters 7: 623631.

Lüttge U. 2004. Ecophysiology of crassulacean acid metabolism (CAM). Annual of Botany 93: $629-652$.

Lüttge U. 2006. Photosynthetic flexibility and ecophysiological plasticity: questions and lessons from Clusia, the only CAM tree, in the neotropics. New Phytologist 171: 7-25.

Mack RN, Pyke DA. 1983. The Demography of Bromus Tectorum: Variation in Time and Space, Journal of Ecology 71: 69-93.

Maestre FT, Bowker MA, Puche MD, Hinojosa MB, et al. 2009. Shrub encroachment can reverse desertification in semiarid Mediterranean grasslands. Ecology Letters 12: 930-941. Maestre FT, Callaway RM, Valladares F, Lortie CJ. 2009. Refining the stress-gradient hypothesis for competition and facilitation in plant communities. Journal of Ecology 97: 199205. 
Manzoni S, Vico G, Porporato A, Katul G. 2013. Biological constraints on water transport in the soil-plant-atmosphere system. Advance in Water Resources 51: 292-304.

Marshall VM, Lewis MM, Ostendorf B. 2012. Buffel grass (Cenchrus ciliaris) as an invader and threat to biodiversity in arid environments: a review. Journal of Arid Environments 78: $1-12$.

Mashiri FE, McClaran MP, Fehmi JS. 2008. Long-term vegetation change related to grazing systems, precipitation and mesquite cover. Rangeland Ecology Management 61: 368-379. May RM. 1977. Thresholds and breakpoints in ecosystems with a multiplicity of stable state. Nature 269: 471-477.

Mata-Gonzalez R, Figueroa-Sandoval B, Clemente F, Manzano M. 2007. Vegetation changes after livestock grazing exclusion and shrub control in the southern Chihuahuan Desert. Western North American Naturalist 67: 63-70.

McClaran MP, Angell DL. 2006. Long-term vegetation response to mesquite removal in desert grassland. Journal of Arid Environment 66: 686-697.

McCulley R, Jobbagy E, Pockman W, Jackson R. 2004. Nutrient uptake as a contributing explanation for deep rooting in arid and semi-arid ecosystems. Oecologia 141: 620-628. McCown RL, Williams WA. 1968. Competition for nutrients and light between the annual grassland Species Bromus Mollis and Erodium Botrys. Ecology 49: 981-990.

McDonald CJ, McPherson GR. 2011. Fire behavior characteristics of buffelgrass-fueled fires and native plant community composition in invaded patches. Journal of Arid Environment 75: 11471154. 
Medina E, Olivares E, Diaz M. 1986. Water stress and light intensity effects on growth and nocturnal acid accumulation in a terrestrial CAM bromeliad (Bromelia humilis Jacq.) under natural conditions. Oecologia 70: 441-446.

Meinzer F, Brooks J, Bucci S, Goldstein G, Scholz F, Warren J. 2004. Converging patterns of uptake and hydraulic redistribution of soil water in contrasting woody vegetation types. Tree Physiology 24: 919-928.

Michalet R. 2003. A test of the indirect facilitation model in a temperate hardwood forest of the northern French Alps. Journal of Ecology 91: 932-940.

Midgley JJ. 1996. Why the world's vegetation is not totally dominated by resprouting plants; because resprouters are shorter than reseeders. Ecography 19:92-95.

Millennium Ecosystem Assessment. 2005, Ecosystems and Human Well-Being: Synthesis. Washington, DC, Island Press.

Miller TE. 1994. Direct and indirect species interactions in an early old-field plant community. American Naturalist 143: 1007-1025.

Miller G, Friedel M, Adam P, Chewings V. 2010. Ecological impacts of buffel grass (Cenchrus ciliaris L.) invasion in central Australia - Does field evidence support a fire-invasion feedback? Rangeland Journal 32: 353-365.

Miller TEX, Louda SM, Rose KA, Eckberg JO. 2009. Impacts of insect herbivory on cactus population dynamics: experimental demography across an environmental gradient. Ecological monographs 79: 155-172.

Miszalski Z, Kornas A, Rozpądek P, Fischer-Schliebs E, Lüttge U. 2013. Independent fluctuations of malate and citrate in the CAM species Clusia hilariana Schltdl. under low light and high light in relation to photoprotection. Journal of Plant Physiology 170: 453-458. 
Moleele NM, Ringrose S, Matheson W, Vanderpost C. 2002. More woody plants? The status of bush encroachment in Botswana's grazing areas. Journal of Environmental Management 64: 311.

Mooney HA, Cleland EE. 2001. The evolutionary impact of invasive species. Proceedings of the National Academy of Sciences of the United States of America 98: 5446-5451.

Mooney HA, Gulmon S, Rundel PW, Ehleringer J. 1980. Further observations on the water relations of Prosopis tamarugo of the northern Atacama desert. Oecologia 44: 177-180.

Mordelet P, Menaut JC. 1995. Influence of trees on above-ground production dynamics of grasses in a humid savanna Journal of Vegetation Science 6: 223-228.

Moreira B, Tormo J, Pausas JG. 2012. To resprout or not to resprout: factors driving intraspecific variability in resprouting. Oikos 121: 1577-1584.

Morgan JA, LeCain DR, Mosier AR, Milchunas DG. 2001. Elevated $\mathrm{CO}_{2}$ enhances water relations and productivity and affects gas exchange in $\mathrm{C}_{3}$ and $\mathrm{C}_{4}$ grasses of the Colorado shortgrass steppe. Global Change Biology 7: 451-466.

Morgan JA, Milchunas DG, LeCain DR, West M, Mosier AR. 2007. Carbon dioxide enrichment alters plant community structure and accelerates shrub growth in the shortgrass steppe. Proceedings of the National Academy of Sciences of the United States of America 104: 1472414729.

Moustakas A, Kunin WE, Cameron TC, Sankaran M. 2013. Facilitation or Competition? Tree Effects on Grass Biomass across a Precipitation Gradient. PLoS One, 8(2), e57025.

Munson SM, Muldavin EH, Belnap J, Peters DPC, Anderson JP, Reiser MH, Melgoza-Castillo A, Herrick JE, Christiansen TA. 2013. Regional signatures of plant response to drought and elevated temperature across a desert ecosystem. Ecology 94: 2030-2041. 
Munson SM, Webb RH, Belnap J, Hubbard JA, Swann DE, Rutman S. 2012. Forecasting climate change impacts to plant community composition in the Sonoran Desert region. Global Chang Biology 18: 1083-1095.

Murphy BP, Bowman DMJS. 2012. What controls the distribution of tropical forest and savanna? Ecology Letters 15: 748-758.

NG GHC, Bedford DR, Miller DM. 2015. Identifying multiple time scale rainfall controls on Mojave Desert ecohydrology using an integrated data and modeling approach for Larrea tridentate. Water Resources Research 51: 3884-3899.

Nippert JB, Wieme RA, Ocheltree TW, Craine JM. 2012. Root characteristics of $\mathrm{C}_{4}$ grasses limit reliance on deep soil water in tallgrass prairie. Plant and Soil 355: 385-394.

Niu SL, Liu WX, Wan SQ. 2008. Different growth response of $\mathrm{C}_{3}$ and $\mathrm{C}_{4}$ grasses to seasonal water and nitrogen regimes and competition in a pot experiment. Journal of Experimental Botany 59: 1431-1439.

Nelson EA, Sage RF. 2008. Functional constraints of CAM leaf anatomy: tight cell packing is associated with increased CAM function across a gradient of CAM expression. Journal of Experimental Botany 59: 1841-1850.

Neumann RB, Cardon ZG. 2012. The magnitude of hydraulic redistribution by plant roots: a review and synthesis of empirical and modeling studies. New Phytologist 194: 337-352.

Nicholson SE. 1980. The Nature of Rainfall Fluctuations in Sub-Tropical West-Africa. Monthly Weather Review 108: 473-487.

Nippert JB, Wieme RA, Ocheltree TW, Craine JM. 2012. Root characteristics of $\mathrm{C}_{4}$ grasses limit reliance on deep soil water in tallgrass prairie. Plant and Soil 355: 385-394. 
Nobel PS. 1983. Nutrient levels in cacti-relation to nocturnal acid accumulation and growth. American Journal of Botany 70: 1244-1253.

Nobel PS. 1996. Responses of some North American CAM plants to freezing temperatures and doubled $\mathrm{CO}_{2}$ concentrations: implications of global climate change for extending cultivation. Journal of Arid Environment 34: 187-196.

Nobel PS. 2003. Environmental biology of agaves and cacti: Cambridge University Press. Nobel PS, Israel AA, Wang N. 1996. Growth, CO2 uptake, and responses of the carboxylating enzymes to inorganic carbon in two highly productive CAM species at current and doubled $\mathrm{CO}_{2}$ concentrations. Plant Cell and Environment 19: 585-592.

Nobel PS, Sanderson J. 1984. Rectifier-like activities of roots of two desert succulents. Journal of Experimental Botany 35: 727-737.

North GB, Nobel PS. 1995. Hydraulic conductivity of concentric root tissues of Agave deserti Engelm. under wet and drying conditions. New Phytologist 130: 47-57.

Noy-Meir I. 1973. Desert ecosystems: Environments and producers. The Annual Review of Ecology, Evolution, and Systematics 4: 25-51.

Noy-Meir I. 1975. Stability of grazing systems: an application of predator-prey graphs. Journal of Ecology 63: 459-481.

Oba G, Post E, Syvertsen PO, Stenseth NC. 2000. Bush cover and range condition assessments in relation to landscape and grazing in southern Ethiopia. Landscape Ecology 15: 535-546.

Ogburn R, Edwards EJ. 2010. The ecological water-use strategies of succulent plants. Advance in Botany Research 55: 179-225. 
O'Halloran LR. 2007. Nutrient and light limitations on vegetation dynamics in the savannas of southern Africa, Ph.D. thesis, Department of Environmental Sciences, University of Virginia, Charlottesville, Virginia, USA.

Okin GS, Herrick JE, Gillette DA. 2006. Multiscale controls on and consequences of aeolian processes in landscape change in arid and semiarid environments. Journal of Arid Environment 65: 253-275.

Okin GS, Parsons AJ, Wainwright J, Herrick JE, Bestelmeyer BT, Peters DPC, Fredrickson EL. 2009a. Do changes in connectivity explain desertification? BioScience 59: 237-244.

Okin GS, D'Odorico P, Archer SR. 2009b. Impacts of feedbacks on Chihuahuan Desert grasslands: transience and metastability driven by grass recruitment. Journal of Geophysical Research 114: G01004.

Olsson AD, Betancourt J, McClaran MP, Marsh SE. 2012. Sonoran Desert Ecosystem transformation by a $\mathrm{C}_{4}$ grass without the grass/fire cycle. Diversity Distribution. 18: 10-21. Osmond CB. 1978. Crassulacean acid metabolism: a curiosity in context. Annual Review of Plant Physiology 29: 379-414.

Ota K. 1988. Stimulation of CAM photosynthesis in Kalanchoe blossfeldiana by transferring to nitrate-deficient conditions. Plant Physiology 87: 454-457.

Owen NA, Fahy KF, Griffiths H. 2009. Crassulacean acid metabolism (CAM) offers sustainable bioenergy production and resilience to climate change. GCB Bioenergy 60: 2879-2896.

Pagès J-P, Pache G, Joud D, Magnan N, Michalet R. 2003. Direct and indirect effects of shade on four forest tree seedlings in the French Alps. Ecology 84: 2741-2750. 
Parsons AJ, Wainwright J, Schlesinger WH, Abrahams AD. 2003. The role of overland flow in sediment and nitrogen budgets of mesquite dunefields, southern New Mexico. Journal of Arid Environments 53: 61-71.

Parton WJ, Ojima DS, Schimel D. 1994. Environmental change in grasslands: assessment using models. Climate Change 28: 111-141.

Paul MJ, Cockburn W. 1990. The stimulation of CAM activity in Mesembryanthemum

crystallinum in nitrate- and phosphate-deficient conditions. New Phytologist 114: 391-398.

Peake DCI, Henzell EF, Stirk GB, Peake A. 1979. Simulation of changes in herbage biomass and drought response of a buffel grass (Cenchrus ciliaris cv. biloela) in Southern Queensland. AgroEcosystems 5: 23-40.

Petrie MD, Collins SL, Gutzler DS, Moore DM. 2014. Regional trends and local variability in monsoon precipitation in the northern Chihuahuan Desert, USA. Journal of Arid Environment 103: $63-70$.

Piepenbrock M, Schmitt JM. 1991. Environmental control of phosphonol pyruvate carboxylase induction in mature. Plant Physiology 97: 998-1003.

Poorter H, Navas ML. 2003. Plant growth and competition at elevated $\mathrm{CO}_{2}$ : on winners, losers and functional groups. New Phytologist. 157, 175-198.

Poorter H, Niklas KJ, Reich PB, Oleksyn J, Poot P, Mommer L. 2011. Biomass allocation to leaves, stems and roots: meta-analyses of interspecific variation and environmental control. New Phytologist 193: 30-50.

Porporato A, D’Odorico P, Laio F, Ridolfi L, Rodriguez-Iturbe I. 2002. Ecohydrology of watercontrolled ecosystems. Advance in Water Resources 25: 1335-1348. 
Porporato A, Laio F, Ridolfi L, Caylor KK, Rodriguez-Iturbe I. 2003. Soil moisture and plant stress dynamics along the Kalahari precipitation gradient. Journal of Geophysical Research 108: D34127.

Porporato A, Vico G, Fay PA. 2006. Superstatistics of hydroclimatic fluctuations and interannual ecosystem productivity. Geophysical of Research Letters 33: L15402.

Prieto I, Armas C, Pugnaire FI. 2012. Water release through plant roots: new insights into its consequences at the plant and ecosystem level. New Phytologist 193: 830-841.

Quijano JC, Kumar P, Drewry DT, Goldstein A, Misson L. 2012. Competitive and mutualistic dependencies in multispecies vegetation dynamics enabled by hydraulic redistribution. Water Resources Research 48:W05518

Quijano JC, Kumar P, Drewry DT. 2013. Passive regulation of soil biogeochemical cycling by root water transport. Water Resource Research 49: 1-18.

Rango A, Huenneke L, Buonopane M, Herrick JE, Havstad KM. 2005. Using historic data to assess effectiveness of shrub removal in southern New Mexico, Journal of Arid Environment 62: 75-91.

Ratajczak Z, Nippert JB, Briggs JM, Blair JM. 2014. Fire dynamics distinguish grasslands, shrublands and woodlands as alternative attractors in the Central Great Plains of North America. Journal of Ecology 102: 1374-1385.

Ravi S, D'Odorico P, Collins SL, Huxman TE. 2009. Can biological invasions induce desertification? New Phytologist 181: 512-515.

Rayder L, Ting IP. 1981. Carbon metabolism in two species of Pereskia (Cactaceae). Plant Physiology 68: 139-142. 
Reich PB. 2014. The world-wide 'fast-slow' plant economics spectrum: a traits manifesto.

Journal of Ecology 102: 275-301.

Reichstein M, Bahn M, Ciais P et al. 2013. Climate extremes and the carbon cycle. Nature 500: $287-295$.

Reyer CPO, Leuzinger S, Rammig A et al. 2012. A plant's perspective of extremes:

terrestrial plant responses to changing climatic variability. Global Change Biology 19: 75-89.

Reyes-García C, Andrade JL. 2009. Crassulacean acid metabolism under global climate change. New Phytologist 181: 754-757.

Reyes-Olivas A, García-Moya E, López-Mata L. 2002. Cacti-shrub interactions in the coastal desert of northern Sinaloa, Mexico. Journal of Arid Environment 52: 431-445.

Richards JH, Caldwell MM. 1987. Hydraulic lift: substantial nocturnal water transport between soil layers by Artemisia tridentata roots. Oecologia 73: 486-489.

Riginos C, Grace JB, Augustine DJ, Young TP. 2009. Local versus landscape-scale effects of savanna trees on grasses. Journal of Ecology 97: 1337-1345.

Ripley BS, Abraham T, Klak C, Cramer MD. 2013. How succulent leaves of Aizoaceae avoid mesophyll conductance limitations of photosynthesis and survive drought. Journal of Experimental Botany 64: 5485-5496.

Rivero RM, Kojima M, Gepstein A, Sakakibara H, Mittler R, Gepstein S, Blumwal E. 2007. Delayed leaf senescence induces extreme drought tolerance in a flowering plant. Proceedings of the National Academy of Sciences of the United States of America 104: 19631-19636.

Rocha FS, Duarte, Waechter JL, Duarte LDS. 2015. Positive association between Bromelia balansae (Bromeliaceae) and tree seedlings on rocky outcrops of Atlantic forest. Journal of Tropical Ecology 31: 195-198. 
Rodriguez-Iturbe I, D'odorico P, Porporato A, Ridolfi L. 1999a. On the spatial and temporal links between vegetation, climate, and soil moisture. Water Resources Research 35: 3709-3722. Rodriguez-Iturbe I, D'odorico P, Porporato A, Ridolfi L. 1999b. Tree-grass coexistence in savannas: The role of spatial dynamics and climate fluctuations. Geophysical Research Letters 26: $247-250$.

Rousset O, Lepart J. 2000. Positive and negative interactions at different life stages of a colonizing species (Quercus humilis). Journal of Ecology 88: 401-412.

Ryel R, Caldwell M, Yoder C, Or D, Leffler A. 2002. Hydraulic redistribution in a stand of Artemisia tridentata: evaluation of benefits to transpiration assessed with a simulation model. Oecologia 130: 173-184.

Ryel RJ, Leffler A, Peek M, Ivans C, Caldwell M. 2004. Water conservation in Artemisia tridentata through redistribution of precipitation. Oecologia 141: 335-345.

Runyan CW, D’Odorico P, Lawrence D. 2012. Physical and biological feedbacks on deforestation. Review of Geophysical 50, RG4006.

Sage RF, McKown AD. 2006. Is $\mathrm{C}_{4}$ photosynthesis less phenotypically plastic than $\mathrm{C}_{3}$ photosynthesis? Journal of Experimental Botany 57: 303-317.

Sala OE, Golluscio RA, Lauenroth WK, Soriano A.1989. Resource partitioning between shrubs and grasses in the Patagonian steppe. Oecologia 81: 501-505.

Sala OE, et al. 2000. Global biodiversity scenarios for the year 2100. Science 287: 1770-1774. Saneoka H, Moghaieb REA, Premachandra GS, Fujita K. 2004. Nitrogen nutrition and water stress effects on cell membrane stability and leaf water relations in Agrostis palustris Huds. Environmental Experimental Botany 52: 131-138. 
Sankaran M, Hanan NP, Scholes RJ, Ratnam J, Augustine DJ, et al. 2005. Determinants of woody cover in African savannas. Nature 438: 846-849.

Sankaran M, Ratnam J, Hanan NP. 2004. Tree-grass coexistence in savannas revisited: insights from an examination of assumptions and mechanisms invoked in existing models. Ecology Letters 7: 480-490.

Sarmiento G. 1984. The ecology of Neotropical savannas. Harvard University Press, Cambridge, MA.

Scanlon, TM, Albertson JD. 2003. Inferred controls on tree/grass composition in a savanna ecosystem: Combining 16 year normalized difference vegetation index data with a dynamic soil moisture model. Water Resources Research 39: 1224.

Scanlon TM, Albertson JD. 2004a. Canopy scale measurements of $\mathrm{CO}_{2}$ and water vapor exchange along a precipitation gradient in southern Africa, Global Change Biology 10(3): 329341.

Scanlon TM, Albertson JD. 2004b. SAFARI 2000 Kalahari Transect $\mathrm{CO}_{2}$, Water Vapor, and Heat Flux, Wet Season 2000, Data set, Available on-line [http://daac.ornl.gov/] from Oak Ridge National Laboratory Distributed Active Archive Center, Oak Ridge, Tennessee, U.S.A. Scheiter S, Higgins SI. 2007. Partitioning of root and shoot competition and the stability of savannas. American Naturalist 170: 587-601.

Schenk HJ, Jackson RB. 2002. Rooting depths, lateral root spreads and below-ground/aboveground allometries of plants in water-limited ecosystems. Journal of Ecology 90: 480-494. Schenk HJ, Jackson RB. 2005. Mapping the global distribution of deep roots in relation to climate and soil characteristics. Geoderma, 126: 129-140. 
Schlesinger WH, Reynolds JF, Cunningham GI, Huenneke LF, Jarrell WM, Virginia RA, Whitford WG. 1990. Biological feedbacks in global desertification. Science 247: 1043-1048. Schmitt JM. 1990. Rapid concentration changes of phosphoenolpyruvate carboxylase mRNA in detached leaves of Mesembryanthemum crystallinum $L$. in response to wilting and rehydration. Plant, Cell and Environment 13: 845-850.

Scholes RJ, Archer SR. 1997. Tree-grass interactions in savannas. Annual Review of Ecology, Evolution, and Systematics 28: 517-544.

Scholes RJ, Dowty PR, Caylor K, Parsons DAB, Frost PGH, Shugart HH. 2002. Trends in savanna structure and composition along an aridity gradient in the Kalahari. Journal of Vegetation Science 13(3): 419-428.

Scholes RJ, Walker BH. 1993. An African savanna: synthesis of the Nylsvley study, Cambridge University Press, New York.

Scholz FG, Bucci SJ, Hoffmann WA, Meinzer FC, Goldstein G. 2010. Hydraulic lift in a Neotropical savanna: experimental manipulation and model simulations. Agricultural and Forest Meteorology 150: 629-639.

Scott RL, Cable WL, Hultine KR. 2008. The ecohydrologic significance of hydraulic redistribution in a semiarid savanna. Water Resour Research 44: W02440.

Seager R, Ting M, Held I, Kushnir Y, Lu J, Vecchi G, Huang HP et al. 2007. Model projections of an imminent transition to a more arid climate in Southwestern North America. Science 16: $1181-1184$.

Siemann E, Rogers WE. 2003. Changes in light and nitrogen availability under pioneer trees may indirectly facilitate tree invasions of grasslands. Journal of Ecology 91: 923-931. 
Smit GN, Rethman NFG. 2000. The influence of tree thinning on the soil water in a semi-arid savanna of southern Africa. Journal of Arid Environment 44: 41-59.

Smith JAC, Winter K. 1996. Taxonomic distribution of Crassulacean acid metabolism.

Crassulacean Acid Metabolism. In: Winter K, Smith JAC, eds. Crassulacean acid metabolism: biochemistry, ecophysiology and evolution. Berlin, Germany: Springer-Verlag, 427-436.

Smith MD. 2011. An ecological perspective on extreme climatic events: a synthetic definition and framework to guide future research. Journal of Ecology 99: 656-663.

Smith SD, Huxman TE, Zitzer SF, Charlet TN, Housman DC, Coleman JS et al. 2000. Elevated $\mathrm{CO}_{2}$ increases productivity and invasive species success in an arid ecosystem. Nature 408: 7981.

Sorte CJ, Ibáñez I, Blumenthal DM, Molinari NA, Miller LP, Grosholz ED, Diez JM, D'Antonio CM, Olden JD, Jones SJ. 2013. Poised to prosper? A cross-system comparison of climate change effects on native and non-native species performance. Ecology Letter 16: 261-270.

Staver AC, Archbald S, Levin AS. 2011a. The global extent and determinants of savanna and forest as alternative biome states, Science, 334, 230-232, doi: 10.1126/science.1210465.

Staver AC, Archbald S, Levin AS. 2011b. Tree cover in sub-Saharan Africa: rainfall and fire constrain savanna and forest as alternative stable states. Ecology 92: 1063-1072.

Staver AC, Levin AS. 2012. Integrating theoretical climate and fire effects on savanna and forest systems. American Naturalist 180: 211-224.

Sternberg LSL, Bucci, S, Franco AC, Goldstein G, Hoffmann WA, Moreira MZ, Scholz F. 2005. Long range lateral root activity by Neo-tropical savanna trees. Plant and Soil 270: 169-178.

Stevens JT, Beckage B. 2009. Fire feedbacks facilitate invasion of pine savannas by Brazilian pepper (Schinus terebinthifolius). New Phytologist 184: 365-375. 
Stone L, Roberts A. 1991. Conditions for a species to gain advantage from the presence of competitors. Ecology: 1964-1972.

Sun XM, Yu KL, Shugart H, Wang G. 2015. Species richness loss after nutrient additions as affected by N: C ratio and plant endogenous hormones in an alpine meadow. Journal of Plant Ecology 2015:rtv037.

Suttle KB, Thomsen MA, Power ME. 2007. Species interactions reverse grassland responses to changing climate. Science 315: 640-642.

Szarek SR, Troughton JH. 1976. Carbon Isotope Ratios in Crassulacean Acid Metabolism Plants Seasonal Patterns from Plants in Natural Stands. Plant Physiology 58: 367-370.

Teuling AJ, Seneviratne SI, Stockli R et al. 2010. Contrasting response of European forest and grassland energy exchange to heatwaves. Nature Geosciences 3: 722-727.

Tews J, Esther A, Milton SJ, Jeltsch F. 2006. Linking a population model with an ecosystem model: assessing the impact of land use and climate change on Savanna shrub cover dynamics. Ecological Modeling 195: 219-228.

Tews J, Schurr F, Jeltsch F. 2004. Seed dispersal by cattle may cause shrub encroachment of Grewia flava on southern Kalahari rangelands. Applied Vegetation Science 7: 89-102. Theoharides KA, Dukes JS. 2007. Plant invasion across space and time: factors affecting nonindigenous species success during four stages of invasion. New Phytologist 176: 256-273. Tilman D. 1982. Resource Competition and Community Structure. Princeton University Press. 1: $1-296$.

Tilman D. 1988. Dynamics and Structure of Plant Communities. Princeton University Press..Princeton, NJ.

Tilman D, Haddi A. 1992. Drought and biodiversity in grasslands. Oecologia 89: 257-264. 
Ting IP. 1981. Effects of abscisic acid on CAM in Portulacaria afra. Photosynth Res 2: 39-48. Toledo M, Poorter L, Peña-Claros M, Alarcón A, Balcázar J, Leaño C, Licona JC, Llanque O, et al. 2011. Climate is a stronger driver of tree and forest growth rates than soil and disturbance. Journal of Ecology 99: 254-264.

Van Auken OW. 2000. Shrub invasions of North American semiarid grasslands. Annual Review of Ecology, Evolution, and Systematics 31: 197-215.

Van der Waal C, de Kroon H, de Boer WF, Heitkonig IMA, Skidmore AK, de Knegt HJ, et al. 2009. Water and nutrients alter herbaceous competitive effects on tree seedlings in a semiarid savanna. Journal of Ecology 97: 430-439.

Vandermeer J. 1990. Indirect and diffuse interactions: complicated cycles in a population embedded in a large community. Journal of Theoretical Biology 142:429-442.

Van Duren IC, Pegtel DM. 2000. Nutrient limitations in wet, drained and rewetted fen meadows: evaluation of methods and results. Plant and Soil 220: 35-47.

van Langevelde F, van de Vijver C, Kumar L, van de Koppel J, de Ridder N, van Andel J, Skidmore AK et al. 2003. Effects of fire and herbivory on the stability of savanna ecosystems, Ecology 84: 337-350.

Van Schaik AH, Struik PC, Damian TG. 1997. Effects of irrigation and N on the vegetative growth of Aloe barbadensis Mill, Aruba. Tropical Agriculture 74: 104-109.

van Wijk MT, Rodriguez-Iturbe I. 2002. Tree-grass competition in space and time: insights from a simple cellular automata model based on ecohydrological dynamics. Water Resources Research 38: 1811-1815. 
Van Wilgen BW, Biggs HC, O’Regan S, Mare N. 2000. A fire history of the savanna ecosystems in the Kruger National Park, South Africa between 1941 and 1996. South African Journal of Science 96: 167-178.

Valiente-Banuet A, Vite F and Zavala-Hurtado JA. 1991. Interaction between the cactus Neobuxbaumia tetetzo and the nurse shrub Mimosa luisana. Journal of Vegetation Science 2:1114. Vaughn KJ, Biel C, Clary JJ, de Herralde F, Aranda X, Evans RY, Young TP, Savé R. 2011. California perennial grasses are physiologically distinct from both Mediterranean annual and perennial grasses. Plant and Soil 345: $37-46$.

Vernon DM, Ostrem JA, Schmitt JM, Bohnert HJ. 1988. PEPCase transcript levels in Mesembryanthemum crystallinum decline rapidly upon relief from salt stress. Plant Physiology 86: $1002-1004$.

Vesk PA, Warton DI, Westoby M. 2004. Sprouting by semi-arid plants: testing a dichotomy and predictive traits. Oikos 107: 72-89.

Vico G, Thompson S, Manzoni S, Molini A, et al. 2015. Climatic, ecophysiological and phenological controls on plant ecohydrological strategies in seasonally dry ecosystems. Ecohydrology 8: 660-681.

Vitousek PM, Mooney HA, Lubchenco J, Melillo JM. 1997. Human domination of Earth's ecosystems. Science 277: 494-499.

Vivrette NJ, Muller CH. 1977. Mechanism of Invasion and Dominance of Coastal Grassland by Mesembryanthemum crystallinum. Ecological Monographs 47: 301-318. 
Volaire F, Thomas H, Lelievre F. 1998. Survival and recovery of perennial forage grasses under prolonged Mediterranean drought: I. Growth, death, water relations and solute content in herbage and stubble. New Phytologist 140: 439-449.

Volder A, Tjoelker MG, Briske DD. 2010. Contrasting physiological responsiveness of establishing trees and a $\mathrm{C}_{4}$ grass to rainfall events, intensified summer drought, and warming in oak savanna. Glob Change Biology 16: 3349-3362.

von Caemmerer S, Griffiths H. 2009. Stomatal responses to $\mathrm{CO}_{2}$ during a diel Crassulacean acid metabolism cycle in Kalanchoe daigremontiana and Kalanchoe pinnata. Plant Cell \& Environmental 32: 567-576.

Walter H. 1971. Ecology of tropical and subtropical vegetation, Oliver \& Boyd, Edinburgh.

Walther GR, Post E, Convey P, Menzel A, Parmesan C, Beebee TJC, Fromentin JM, HoegGuldberg O, Bairlein F. 2002. Ecological responses to recent climate change. Nature 416 : 389-395.

Walker BH, Ludwig D, Holling CS, Peterman RM. 1981. Stability of semiarid savanna grazing systems. Journal of Ecology 69: 473-498.

Walker BH, Noy-Meir I. 1982. Aspects of stability and resilience of savanna ecosystems. Pages 556-590 in B. H. Walker and B. J. Huntley, eds. Ecology of subtropical savannas. Springer, Berlin.

Walter H. 1971. Ecology of tropical and subtropical vegetation. Oliver \& Boyd, Edinburgh. Wang L, D’Odorico P, Macko S, Ringrose S, Coetzee S. 2007. Biogeochemistry of Kalahari sands, Journal of Arid Environment 71: 259-279. 
Wang L, D’Odorico P, Ries L, Caylor K, Macko S. 2009. Combined effect of soil moisture and nitrogen availability variations on grass productivity in African Savannas. Plant and Soil 328: 95-108.

Wang Z, Taub DR. 2010. Interactive effects of elevated carbon dioxide and environmental stress on root mass fraction in plants: a meta-analytic synthesis using pairwise techniques. Oecologia. 163: $1-11$.

Ward D, Wiegand K, Getzin S. 2013. Walter's two-layer hypothesis revisited: back to the roots! Oecologia 172: 617-630.

Warman L, Moles AT. 2009. Alternative stable states in Australia's wet tropics: a theoretical framework for the field data and a field-case for the theory. Landscape Ecology 24: 1-13.

Warren JM, JR Brooks, FC Meinzer, JL Eberhart. 2008. Hydraulic redistribution of water from Pinus ponderosa trees to seedlings: evidence for an ectomycorrhizal pathway. New Phytologist 178: 382-394.

Weltzin JF, McPherson GR. 1997. Spatial and temporal soil moisture resource partitioning by trees and grasses in a temperate savanna, Arizona, USA. Oecologia 112: 156-164.

Williams CA, Albertson JD. 2006. Dynamical effects of the statistical structure of annual rainfall on dryland vegetation. Global Change Biology 12: 777-792.

Winter K, Foster JG, Schmitt MR, Edwards GE. 1982. Activity and quantity of ribulose bisphosphate carboxylase and phosphoenolpyruvate carboxylase-protein in two crassulacean acid metabolism plants in relation to leaf age, nitrogen nutrition, and point in time during a day/night cycle. Planta 154: 309-317. 
Winter K, Garcia M, Holtum JA. 2009. Canopy $\mathrm{CO}_{2}$ exchange of two neotropical tree species exhibiting constitutive and facultative CAM photosynthesis, Clusia rosea and Clusia cylindrica. Journal of Experimental Botany 60: 3167-3177.

Winter K, Holtum JAM. 2007. Environment or Development? Lifetime Net $\mathrm{CO}_{2}$ Exchange and Control of the Expression of Crassulacean Acid Metabolism in Mesembryanthemum crystallinum. Plant Physiology 143: 98-107.

Winter K, Holtum JAM. 2011. Induction and reversal of crassulacean acid metabolism in Calandrinia polyandra: effects of soil moisture and nutrients. Functional Plant Biology 38: 576582.

Winter K, Holtum JAM. 2014. Facultative crassulacean acid metabolism (CAM) plants: powerful tools for unravelling the functional elements of CAM photosynthesis. Journal of Experimental Botany 65: 1-17.

Withgott J. 2000. Botanical Nursing: From deserts to shorelines, nurse effects are receiving renewed attention. BioScience 50: 479-484.

Xu XT, Medvigya D, Rodriguez-Iturbeb, I. 2015. Relation between rainfall intensity and savanna tree abundance explained by water use strategies. Proceedings of the National Academy of Sciences of the United States of America 112: 12992-12996.

Yang HJ, Li Y, Wu MY, Zhang Z, Li LH, Wan SQ. 2011. Plant community responses to nitrogen addition and increased precipitation: the importance of water availability and species traits. Global Change Biology 17: 2936-2944.

Yao J, Peters DPC, Havstad KM, Gibbens RP, Herrick JE. 2006. Multiscale factors and longterm responses of Chihuahuan Desert grasses to drought. Landscape Ecology 21: 1217-1231. 
Yoder C, Nowak R. 1999. Hydraulic lift among native plant species in the Mojave Desert. Plant and Soil 215: 93-102.

Yu KL, D'Odorico P. 2014a. An ecohydrological framework for grass displacement by woody plants in savannas. Journal of Geophysical Research 119: 2013JG002577.

Yu KL, D'Odorico P. 2014b. Climate, vegetation, and soil controls on hydraulic redistribution in shallow tree roots. Advances in water resources 66: 70-80.

Yu KL, D’Odorico P. 2015a. Hydraulic lift as a determinant of tree-grass coexistence on savannas. New Phytologist. 207(4): 1038-51.

Yu KL, D’Odorico P. 2015b. Direct and indirect facilitation of plants with Crassulacean Acid Metabolism (CAM). Ecosystem. 18: 985-999.

Yu KL, D’Odorico P. From facilitative to competitive interactions between woody plants and plants with Crassulacean Acid Metabolism (CAM): the role of hydraulic descent. Ecohydrology (In press)

Zavaleta ES, Shaw MR, Chiariello NR, Thomas BD, Cleland EE, Field CB, Mooney HA. 2003.

Grassland responses to three years of elevated temperature, $\mathrm{CO}_{2}$, precipitation, and $\mathrm{N}$ deposition. Ecological Monographs 73: 585-604.

Zeng N, Neelin JD. 2000. The role of vegetation-climate interaction and interannual variability in shaping the African savanna. Journal of Climate 13: 2665-2670.

Zeng N, Neelin JD, Lau K-M, Tucker CJ. 1999. Enhancement of interdecadal climate variability in the Sahel by vegetation interaction. Science 286: 1537-1540.

Zeppel MJB, Wilks JV, Lewis JD. 2014. Impacts of extreme precipitation and seasonal changes in precipitation on plants. Biogeosciences 11: 3083-3093. 
Ziska LH, Reeves JB, Blank B. 2005. The impact of recent increases in atmospheric $\mathrm{CO}_{2}$ on biomass production and vegetative retention of Cheatgrass (Bromus tectorum): implications for fire disturbance. Global Change Biology 11: 1325-1332.

Zou CB, Barnes PW, Archer S, McMurtry CR. 2005. Soil moisture redistribution as a mechanism of facilitation in savanna tree-shrub clusters. Oecologia 145: 32-40.

Zou CB, Royer PD, Breshears DD. 2010. Density-dependent shading patterns by Sonoran saguaros. Journal of Arid Environment 74: 156-158. 